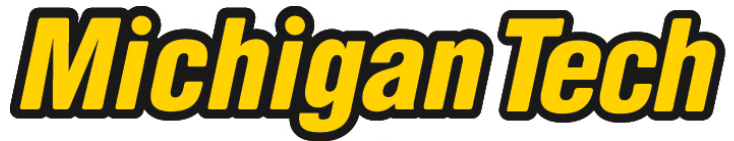 \\ Michigan Technological University Create the Future Digital Commons @ Michigan Tech
}

2012

\section{Evaluation of surface defect detection in reinforced concrete bridge decks using terrestrial LiDAR}

Ryan C. Hoensheid

Michigan Technological University

Follow this and additional works at: https://digitalcommons.mtu.edu/etds

Part of the Civil and Environmental Engineering Commons

Copyright 2012 Ryan C. Hoensheid

\section{Recommended Citation}

Hoensheid, Ryan C., "Evaluation of surface defect detection in reinforced concrete bridge decks using terrestrial LiDAR ", Master's Thesis, Michigan Technological University, 2012.

https://doi.org/10.37099/mtu.dc.etds/244

Follow this and additional works at: https://digitalcommons.mtu.edu/etds

3 Part of the Civil and Environmental Engineering Commons 


\title{
AN EVALUATION OF SURFACE DEFECT DETECTION IN REINFORCED CONCRETE BRIDGE DECKS USING TERRESTRIAL LIDAR
}

\section{By}

Ryan C. Hoensheid

\begin{abstract}
A THESIS
Submitted in partial fulfillment of the requirements for the degree of MASTER OF SCIENCE

(Civil Engineering)
\end{abstract}

MICHIGAN TECHNOLOGICAL UNIVERSITY

2012 
This thesis, "An Evaluation of Surface Defect Detection in Reinforced Concrete Bridge Decks using Terrestrial LiDAR," is hereby approved in partial fulfillment of the requirements for the Degree of MASTER OF SCIENCE CIVIL ENGINEERING.

Department of Civil and Environmental Engineering

Signatures:

Thesis Advisor

Dr. Theresa M. Ahlborn, P.E.

Department Chair

Dr. David W. Hand, P.E.

Date 


\section{TABLE OF CONTENTS}

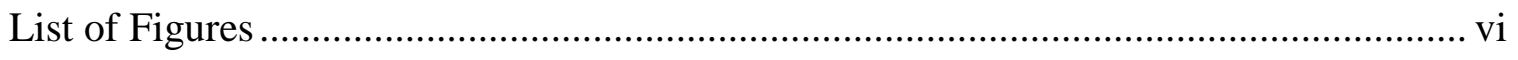

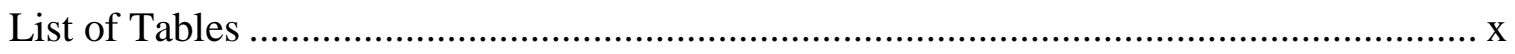

Acknowledgments....................................................................................................... xi

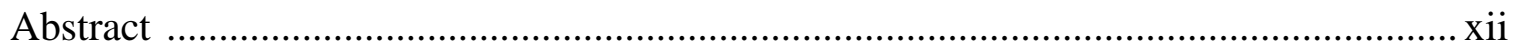

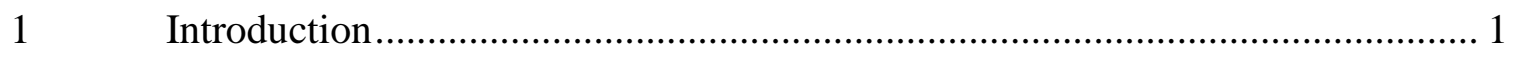

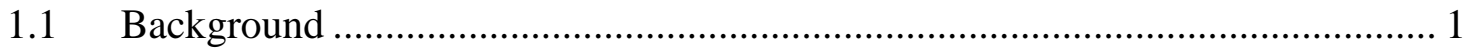

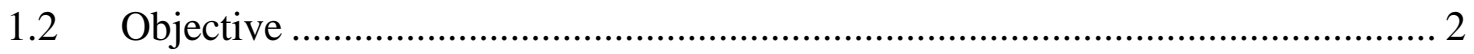

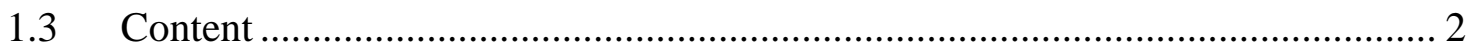

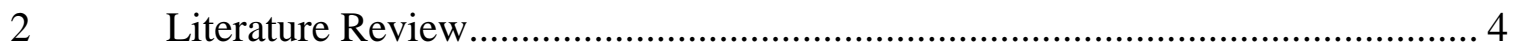

2.1 Challenges for the National Bridge Inventory Infrastructure................................ 4

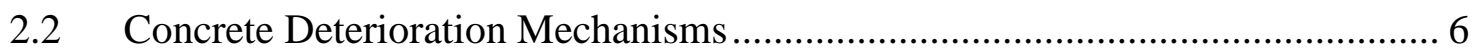

2.3 Development of Present Bridge Inspection Procedures..................................... 12

2.4 Commonly Recognized Structural Elements ..................................................... 14

2.5 Concerns with Standard Bridge Inspection Procedures ....................................... 15

2.6 Advanced Concrete Bridge Inspection Techniques ........................................... 17

2.6.1 Ground-penetrating Radar ...................................................................... 17

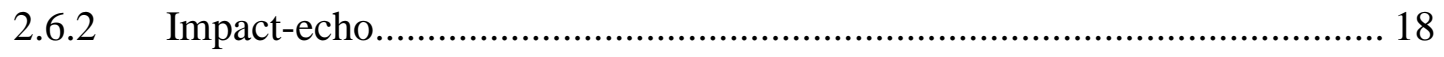

2.6.3 Infrared Thermography ......................................................................... 19

$2.7 \quad$ Fundamentals of LiDAR ............................................................................ 21

2.8 LiDAR Operating Parameters ....................................................................... 25

2.9 Fixed Terrestrial LiDAR Performance Evaluation .............................................. 27

2.10 Current LiDAR Applications in Civil Engineering........................................... 35

2.11 Summary of Literature Review Sections …………………………………….... 35 
$3 \quad$ Methodology .........

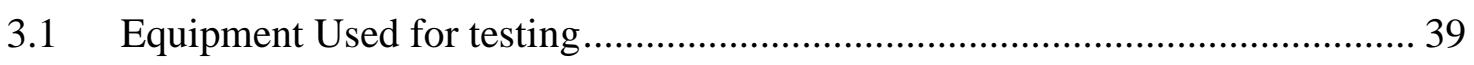

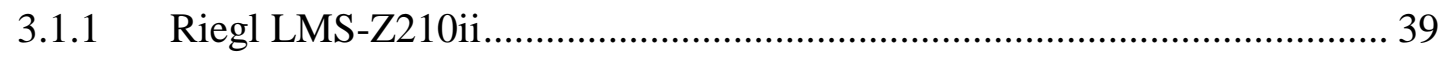

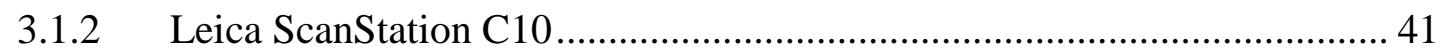

3.2 Full-scale Field Demonstration Selection ............................................... 42

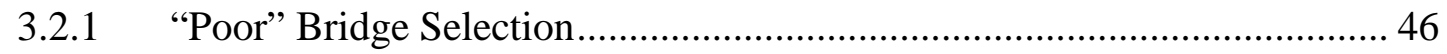

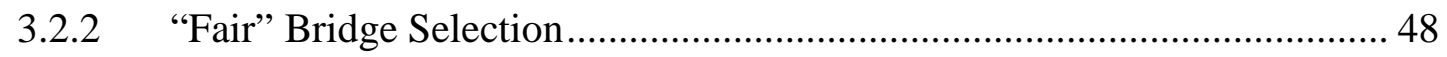

3.2.3 "Satisfactory” Bridge Selection ........................................................ 50

3.2.4 "Supplemental” Bridge Selection ......................................................... 52

3.3 Full-scale Field Demonstration Plan ........................................................ 54

3.4 Post-processing Software Selection ....................................................... 57

3.5 Certainty 3D’s TopoDOT Modeling Software............................................ 59

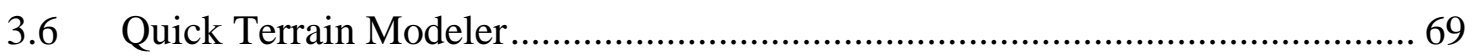

3.7 MTRI 3DOBS Spall Detection Algorithm............................................... 74

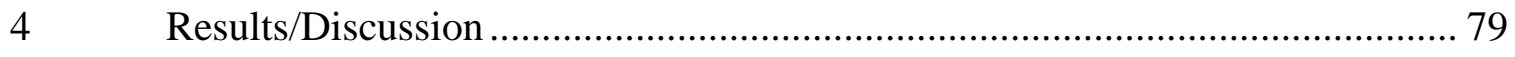

4.1 General Findings from Full-scale Field Demonstration................................. 79

4.1.1 Point Cloud Density Decay ............................................................... 79

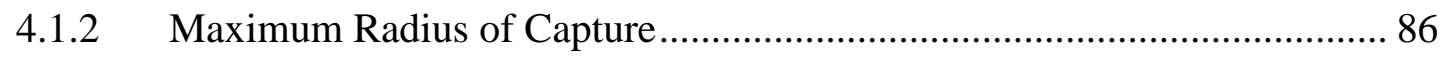

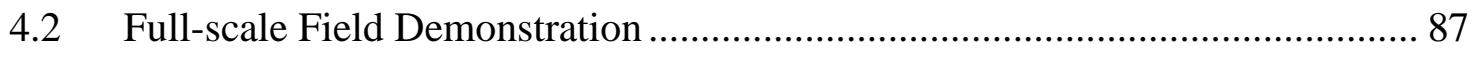

4.2.1 Ground Truth Measure for Comparison ............................................. 88

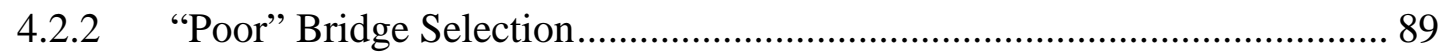

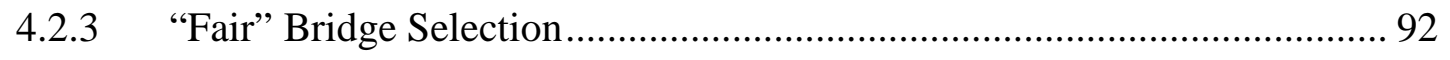

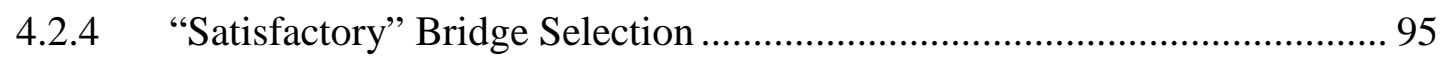

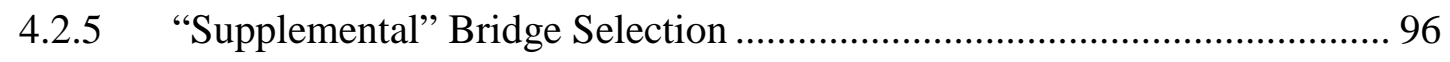




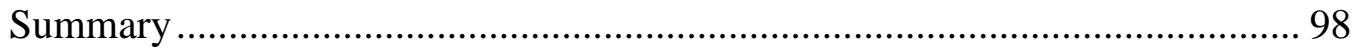

5.1 Sensing Bridge Surface Condition ........................................................... 98

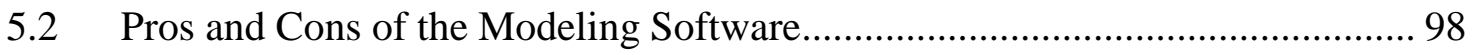

5.2.1 Certainty 3D’s TopoDOT Modeling Software ....................................... 99

5.2.2 Quick Terrain Modeler .................................................................. 100

5.2.3 MTRI 3DOBS Spall Detection Algorithm ......................................... 101

5.3 Implementation of LiDAR for Bridge Inspections ..................................... 102

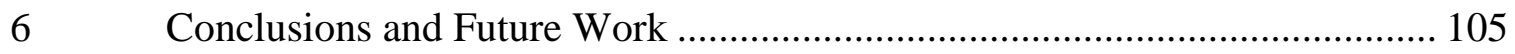

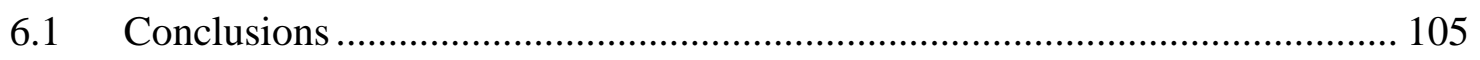

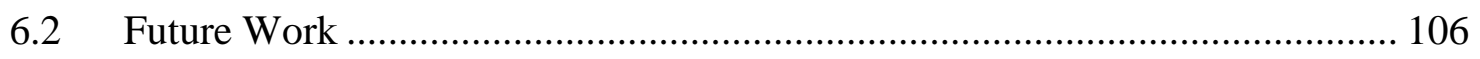

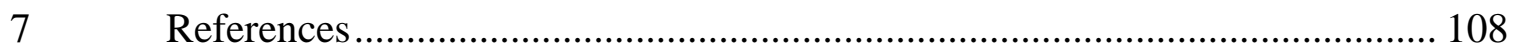

Appendix A: MDOT Field Demonstration Inspection Reports .................................. 112

Appendix B: Leica ScanStation C10 Field Sketches .............................................. 128

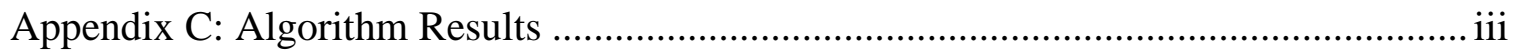

Appendix D: Clarification of Potential Copyrighted Material .................................... 136 


\section{List of Figures}

Figure 2.1: $\quad$ MDOT's RC bridge deck preservation matrix (MDOT 2011a) (See

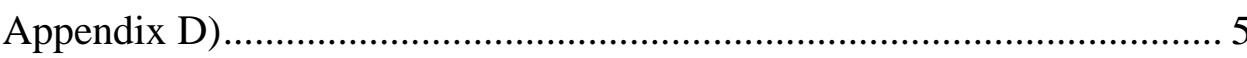

Figure 2.2: $\quad$ Reinforced concrete deterioration diagram................................................. 7

Figure 2.3: Classification of concrete cracks (FHWA 2006) (See Appendix D) .......... 8

Figure 2.4: $\quad$ Example of concrete spalling (Courtesy of Renee Oats) (See Appendix D)

Figure 2.5: Example of concrete scaling (Courtesy of Renee Oats) (See Appendix D) .

Figure 2.6: $\quad$ MDOT performed hammer sounding (Courtesy of Renee Oats) (See Appendix D)...................................................................................... 16

Figure 2.7: $\quad$ MDOT performed chain-drag testing (Courtesy of Renee Oats) (See Appendix D)..................................................................................... 17

Figure 2.8: $\quad$ Hand held impact-echo equipment ………….......................................... 19

Figure 2.9: $\quad$ Optical and thermal image highlighting observable surface defect (Courtesy of Khatereh Vaghefi) (See Appendix D)................................... 21

Figure 2.10: Basic LiDAR function flow chart ............................................................. 22

Figure 2.11: Bistatic vs. monostatic LiDAR configurations ........................................... 24

Figure 2.12: Control test fixtures (Hiremagalur et al. 2007) (See Appendix D) ........... 28

Figure 2.13: Illustration of incidence and coverage angles ............................................ 30

Figure 2.14: Light reflectance spectrum........................................................................ 30

Figure 2.15: Example of laser point cloud resolution test fixture (Leica) at $25 \mathrm{~m}$ and 100 m (Hiremagalur et al. 2007) (See Appendix D)................................... 33

Figure 3.1: $\quad$ Riegl LMS-Z210ii system (Courtesy of Renee Oats) (See Appendix D). 40 Figure 3.2: Leica ScanStation C10 system (Courtesy of MTRI) (See Appendix D) .. 42

Figure 3.3: Field demonstration locations .................................................................. 45 
Figure 3.4: "Poor" bridge selection ………………................................................ 46

Figure 3.5: Mannsiding Road photographs (Courtesy of MTRI) (See Appendix D).. 48

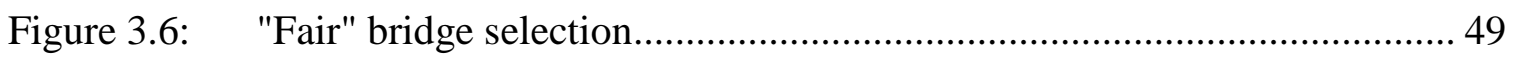

Figure 3.7: Willow Road photograph (Courtesy of MTRI) (See Appendix D) .......... 50

Figure 3.8: "Satisfactory" bridge selection ................................................................. 51

Figure 3.9: $\quad$ Freer Road photographs (Courtesy of MTRI) (See Appendix D) ............ 52

Figure 3.10: "Supplemental" bridge selection .................................................................. 53

Figure 3.11: Mannsiding Road photographs (Courtesy of Renee Oats) (See Appendix

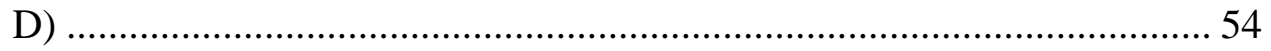

Figure 3.12: Vendor specific targets (Leica to left, Riegl to right) (Courtesy of MTRI) (See Appendix D)..................................................................................... 55

Figure 3.13: Sample LiDAR collection locations (red dots are LiDAR setups) ............ 56

Figure 3.14: Global positioning system for geo-referencing (Courtesy of MTRI) (See Appendix D)...................................................................................... 60

Figure 3.15: TopoDOT initializing user prompt (Certainty 2011) (See Appendix D).. 61

Figure 3.16: TopoDOT’s status text window (Certainty 2011) (See Appendix D) ...... 62

Figure 3.17: MicroStation user interface with TopoDOT activated (Certainty 2011) (See Appendix D).................................................................................... 63

Figure 3.18: Import file settings for establishing import parameters (Certainty 2011) (See Appendix D)................................................................................ 64

Figure 3.19: Design file settings for establishing display parameters (Certainty 2011) (See Appendix D).................................................................................... 64

Figure 3.20: Proper procedure to load desired LAS file (Certainty 2011) (See Appendix

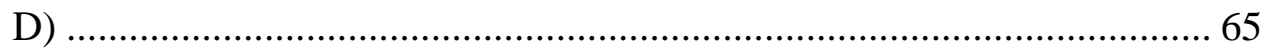

Figure 3.21: Confirming displayable points not exceeded (Certainty 2011) (See Appendix D)........................................................................................ 66 
Figure 3.22: LiDAR data populated user interface (Certainty 2011) (See Appendix D) 67

Figure 3.23: Deck extraction utilizing the fence command (Certainty 2011) (See

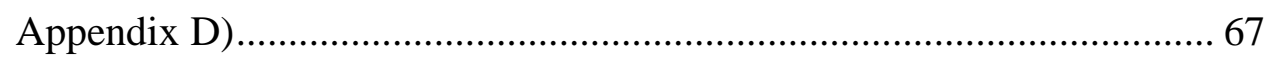

Figure 3.24: Filtered data pertaining to the bridge deck (Certainty 2011) (See Appendix D) 68

Figure 3.25: Exporting filtered deck data (Certainty 2011) (See Appendix D) ............ 69

Figure 3.26: Proper file import method (Imagery 2011) (See Appendix D)................. 70

Figure 3.27: Setting markers at desired section extents (Imagery 2011) (See Appendix D) 71

Figure 3.28: Establishing the Z-Polygon parameters for subset export (Imagery 2011) (See Appendix D). 72

Figure 3.29: $\quad$ Proper exporting procedure for individual bridge subsets (Imagery 2011) (See Appendix D) 72

Figure 3.30: Subset file import for generation of geotiff DEM (Imagery 2011) (See

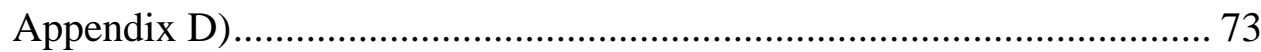

Figure 3.31: Exporting procedure for geotiff DEM file (Imagery 2011) (See Appendix

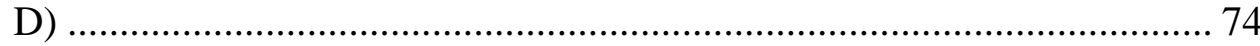

Figure 3.32: $\quad$ MTRI 3DOBS spall detection algorithm user prompt (MTRI 2011) ....... 76

Figure 3.33: Populated working directory from MTRI 3DOBS spall detection algorithm (MTRI 2011) 77

Figure 3.34: Optical visual aid for validation of spall detection process (MTRI 2011) 78

Figure 4.1: $\quad$ Mannsiding Road NB point density texture for visual representation....... 81

Figure 4.2: $\quad$ Mannsiding Road NB longitudinal elevation profile................................. 81

Figure 4.3: $\quad$ Mannsiding Road SB point density texture for visual representation ...... 82

Figure 4.4: $\quad$ Mannsiding Road SB longitudinal elevation profile................................... 82

Figure 4.5: Willow Road point density texture for visual representation .................... 83 
Figure 4.6: Willow Road longitudinal elevation profile........................................ 83

Figure 4.7: $\quad$ Freer Road point density texture for visual representation ...................... 84

Figure 4.8: $\quad$ Freer Road longitudinal elevation profile ........................................... 84

Figure 4.9: Visual representation of the reduction in coverage angle over distance... 85

Figure 4.10: The coverage angle and angle of incidence as the distance varies ........... 86

Figure 4.11: 3 BDOBS field demonstration data collection system (Courtesy of Renee Oats) (See Appendix D) ................................................................ 88

Figure 4.12: Optical image of Mannsiding Road NB showing evaluated spall location (Courtesy of MTRI) (See Appendix D) ............................................... 90

Figure 4.13: Optical image and the predicted spall area image for visual comparison (Courtesy of MTRI) (See Appendix D) .............................................. 91

Figure 4.14: Optical image of Willow Road showing evaluated surface defect location (Courtesy of MTRI) (See Appendix D) ............................................... 94

Figure 4.15: Optical image and the predicted spall area image for visual comparison. 94 


\section{List of Tables}

Table 2.1: $\quad$ Concrete crack width guidelines (FHWA 2006) (See Appendix D) .......... 9

Table 2.2: $\quad$ Concrete crack density guidelines (FHWA 2006) (See Appendix D) ...... 10

Table 2.3: $\quad$ Reinforced concrete bridge deck condition state matrix (AASHTO 2011) (See Appendix D)............................................................................. 15

Table 2.4: $\quad$ RMSE (mm) of range precision at 95\% confidence interval (Hiremagalur et al. 2007) (See Appendix D) ............................................................. 29

Table 2.5: $\quad$ Laser point cloud data of central cross-section (Hiremagalur et al. 2007) (See Appendix D)............................................................................. 34

Table 3.1: $\quad$ Riegl LMS-Z210ii system performance (Riegl 2011) ............................. 41

Table 3.2: $\quad$ Leica ScanStation C10 system performance (Geosystems 2011)............. 41

Table 3.3: $\quad$ NBI rating scale (MDOT 2011b) (See Appendix D) ............................... 44

Table 4.1: $\quad$ Maximum radius of capture for Leica ScanStation C10 at 50,000 points/second ........................................................................... 87

Table 4.2: $\quad$ Results from the Mannsiding Road NB selected deck evaluation ............ 90

Table 4.3: $\quad$ Isolated section within capture radius on Mannsiding Road NB.............. 92

Table 4.4: $\quad$ Results from the Willow Road selected deck evaluation......................... 93

Table 4.5: $\quad$ Isolated section within capture radius on Willow Road .......................... 95

Table 4.6: $\quad$ Results from the Freer Road selected deck evaluation ............................ 96

Table 4.7: $\quad$ Results from the Mannsiding Road SB full deck evaluation .................... 97 


\section{Acknowledgments}

I would like to thank my advisor, Dr. Tess Ahlborn, for providing guidance throughout the process of the project. This work was supported by the Commercial Remote Sensing and Spatial Information Technologies program of the Research and Innovative Technology Administration (RITA), U.S. Department of Transportation (USDOT), Cooperative Agreement \# DTOS59-10-H-00001, with additional support provided by the Michigan Department of Transportation (MDOT), the Michigan Tech Transportation Institute (MTTI), the Michigan Tech Research Institute (MTRI), and the Center for Automotive Research (CAR). I am grateful for the contributions of my committee members Dr. Devin Harris and Mr. Colin Brooks. I greatly appreciate the contributions from all the RITA project members in completing my work, particularly David Dean and Rick Dobson. Finally, I would like to thank my fiancé along with all my family and friends who have supported me throughout this process. The views, opinions, findings, and conclusions reflected in this paper are the responsibility of the author only and do not represent the official policy or position of the USDOT/RITA, or any state or other entity. 


\section{Abstract}

Routine bridge inspections require labor intensive and highly subjective visual interpretation to determine bridge deck surface condition. Light Detection and Ranging (LiDAR) a relatively new class of survey instrument has become a popular and increasingly used technology for providing as-built and inventory data in civil applications. While an increasing number of private and governmental agencies possess terrestrial and mobile LiDAR systems, an understanding of the technology's capabilities and potential applications continues to evolve. LiDAR is a line-of-sight instrument and as such, care must be taken when establishing scan locations and resolution to allow the capture of data at an adequate resolution for defining features that contribute to the analysis of bridge deck surface condition. Information such as the location, area, and volume of spalling on deck surfaces, undersides, and support columns can be derived from properly collected LiDAR point clouds. The LiDAR point clouds contain information that can provide quantitative surface condition information, resulting in more accurate structural health monitoring.

LiDAR scans were collected at three study bridges, each of which displayed a varying degree of degradation. A variety of commercially available analysis tools and an independently developed algorithm written in ArcGIS Python (ArcPy) were used to locate and quantify surface defects such as location, volume, and area of spalls. The results were visual and numerically displayed in a user-friendly web-based decision support tool integrating prior bridge condition metrics for comparison. LiDAR data processing procedures along with strengths and limitations of point clouds for defining features useful for assessing bridge deck condition are discussed. Point cloud density and incidence angle are two attributes that must be managed carefully to ensure data collected are of high quality and useful for bridge condition evaluation. When collected properly to ensure effective evaluation of bridge surface condition, LiDAR data can be analyzed to provide a useful data set from which to derive bridge deck condition information. 


\section{Introduction}

\subsection{Background}

Through the years of hard use and shrinking maintenance funding, the United States’ bridge infrastructure system has been rapidly deteriorating. A significant percentage of the nation's approximately 600,000 bridges are categorized as structurally deficient or functionally obsolete. In fact, the National Bridge Inventory (NBI) database revels that approximately 87,000 bridges possess structurally deficient characteristics (Fuchs et al. 2004). This staggering percentage deemed as structurally deficient will require extensive repair and possible reconstruction, which will exhaust limited resources, but is necessary to ensure safety and reliability. The current bridge inspection standards require bridges to be inspected at least once every two years. If the two year inspection period is deemed as inadequate due visible signs of distress, the inspector can shorten the inspection frequency. This is done mainly through a visual inspection in which the inspector uses their expertise and past experiences to determine the condition of the bridge (FHWA 2006). Visual inspection practices are extremely subjective and lead to highly variable results depending on the inspector. The subjectivity of this process makes it difficult to gain consistent bridge condition assessments. By introducing remote sensing techniques such as Light Detection and Ranging (LiDAR) into the current inspection practice, the variability could be reduced, allowing for accurate determination of defects and precise allocation of appropriate funding. A general definition of remote sensing is the collection and measurement of spatial information about an object, area, or phenomenon at a distance from the data source, without direct contact (Falkner 1995).

The use of LiDAR is a recent development within the civil engineering industry. Currently, the industry utilizes LiDAR technology as a high output, low operational cost survey tool. LiDAR creates an accurate point cloud rendering allowing for bridge inventory measures, work site surveys and as-built construction models. With the continuously narrowing technological gap, LiDAR has advanced tremendously, allowing for the technology to become economically acceptable. Unfortunately, the civil 
engineering industry has faltered, due to the lack of funding, to keep pace with the technological advancements resulting in underutilization of this technology for bridge condition assessment. However, because numerous Department of Transportations (DOTs) are already acquiring and using LiDAR systems as a part of their day-to-day operations, the knowledge and equipment is already available and just requires repurposing for collection of bridge condition metrics.

\subsection{Objective}

The objective of this research was to detect and quantify deterioration of a concrete bridge surface through the application of LiDAR. Several different types of deterioration were considered, including spalling, scaling and cracking, to determine the level to which this technology can accurately sense the concrete surface condition. This research was performed to evaluate the technology's potential to accurately sense topical bridge deck defects by comparing with field demonstration. Capabilities of LiDAR were investigated to incorporate this technology into the present cache of available bridge inspection tools. The most effective and appropriate methods of data presentation to the end-user were also considered.

\subsection{Content}

The present bridge inspection practices and the root causes of the degradation of concrete material are reveled. A common understanding of the basics of LiDAR, the current state within the civil engineering realm of the practice and operating parameters are discussed within chapter two, the literature review.

Four separate field demonstration data collections were conducted to evaluate this technology's applicability in the remote sensing arena as an inspection tool, which is discussed in chapter three. The primary focus of the research was completed on reinforced concrete decks, which is considered a Commonly Recognized (CoRe) Structural Element as defined by the American Association of State Highway and Transportation Officials (AASHTO). Chapter four contains the defect detection results 
from these field demonstrations, which were visually and numerically. Concluding remarks of the technology evaluation and the future work necessary to take this remote sensing application from research form to user ready are presented in chapter six. 


\section{Literature Review}

The United States' highway infrastructure faces an unprecedented deterioration problem. Challenging environmental conditions and increasing traffic volumes make bridges more susceptible than ever to accelerated deterioration (Scott et al. 2002). Current bridge construction practices within the United States utilize numerous types of materials, which include concrete, steel, bituminous, polymer and timber. Of these various types, concrete is the most widely used material for bridge decks. The knowledge of the current state of concrete bridge material spurred the investigation into the application of LiDAR as a remote sensing technology to detect surface defects present on concrete bridge decks. To accurately assess and diagnose bridge conditions, a firm understanding of the current state of the infrastructure system is necessary.

Currently the United States' bridge infrastructure system is in a state of disrepair stemming from years of limited resources and inadequate maintenance. Recent events such as the Minneapolis I-35W collapse and the San Francisco-Oakland Bay Bridge collapse have brought light to this ever growing issue. However, with almost 87,000 bridges within the United States deemed as structurally deficient, 68,000 of which are concrete, these tragedies are only a precursor of events to come (Federal Highway Administration (FHWA) 2010).

\subsection{Challenges for the National Bridge Inventory Infrastructure}

The majority of in-service or planned bridges in the United States utilize a reinforced concrete deck. Additional material such as timber, steel orthotropic, steel grid, composite or polymeric are used as alternative bridge deck materials (Ahlborn et al. 2010). Extensive research is being conducted by various academic, state and federal agencies in regards to the deterioration mechanisms of traditional reinforced concrete material. However, research has yet to develop accurate methods for field inspectors to detect deterioration mechanisms early. This causes the reinforced concrete deck to be 
classified, to a certain extent, as a sacrificial element requiring minimal maintenance and crude methods of condition state evaluation.

During routine bridge inspections, condition states are assigned to individual bridge elements. These condition states are compiled in a bridge safety inspection report (BSIR) allowing for the engineer to efficiently assess the individual components of the bridge and consider potential repair options for deficient elements. Federal and state agencies utilize self-developed preservation matrices to efficiently allocate resources in the effort of bridge preservation. Figure 2.1 shows the Michigan Department of Transportation's (MDOT) reinforced concrete (RC) deck preservation matrix, which classifies deterioration, associated actions required for repair and anticipated service life extension (MDOT 2011a). The matrix generates possible repair options for particular deck condition states, which consists of two categories, top and bottom surfaces.

BRIDGE DECK PRESERVATION MATRIX - DECKS WITH EPOXY COATED REBAR (ECR)

\begin{tabular}{|c|c|c|c|c|c|c|c|c|}
\hline \multicolumn{5}{|c|}{ DECK CONDITION STATE } & \multirow{3}{*}{ REPAIR OPTIONS } & \multicolumn{2}{|c|}{$\begin{array}{c}\text { POTENTIAL RESULT TO } \\
\text { DECK BSIR }\end{array}$} & \multirow{3}{*}{$\begin{array}{l}\text { ANTICIPATED } \\
\text { FIX LIFE }\end{array}$} \\
\hline \multicolumn{3}{|c|}{ Top Surface } & \multicolumn{2}{|c|}{ Bottom Surface } & & & & \\
\hline & BSIR \#58a & $\begin{array}{c}\text { Deficiencies } \\
\% \text { (a) }\end{array}$ & BSIR \#58b & \begin{tabular}{|c|} 
Deficiencies \\
$\%$ (b)
\end{tabular} & & BSIR \#58a & BSIR \#58b & \\
\hline \multirow{3}{*}{\multicolumn{2}{|c|}{$\geq 5$}} & N/A & N/A & N/A & $\begin{array}{c}\text { Hold (c) } \\
\text { Seal Cracks/Healer Sealer (d) }\end{array}$ & No Change & No Change & 1 to 4 years \\
\hline & & $\leq 5 \%$ & $>5$ & $\leq 2 \%$ & Epoxy Overlay & 8,9 & No Change & 10 to 15 years \\
\hline & & $\leq 10 \%$ & $24(k)$ & $\leq 25 \%(\mathrm{k})$ & Deck Patch (e) & Up by $1 \mathrm{pt}$. & No Change & 3 to 10 years \\
\hline \multirow{3}{*}{\multicolumn{2}{|c|}{$4(k)$ or 5}} & \multirow{3}{*}{$\begin{array}{l}10 \% \text { to } \\
25 \%(k)\end{array}$} & \multirow[b]{2}{*}{$4(k)$} & \multirow{2}{*}{$\begin{array}{l}10 \% \text { to } \\
25 \%(k)\end{array}$} & Shallow Concrete Overlay $(h, i)$ & 8,9 & No Change & 20 to 25 years \\
\hline & & & & & $\begin{array}{l}\text { HMA Overlay with water- } \\
\text { proofing membrane }(f, h, i)\end{array}$ & 8,9 & No Change & 8 to 10 years \\
\hline & & & 2 or $3(k)$ & $>25 \%(k)$ & HMA Cap $(g, h, i)$ & 8,9 & No Change & 2 to 4 years \\
\hline \multirow{4}{*}{\multicolumn{2}{|c|}{$\leq 3(k)$}} & \multirow{4}{*}{$>25 \%(k)$} & \multirow{2}{*}{$4(k)$ or 5} & \multirow[b]{2}{*}{$2 \%$ to $25 \%(\mathrm{k})$} & Shallow Concrete Overlay $(h, i)$ & 8,9 & No Change & 10 years \\
\hline & & & & & $\begin{array}{l}\text { HMA Overlay with water- } \\
\text { proofing membrane }(f, h, i)\end{array}$ & 8,9 & No Change & 5 to 7 years \\
\hline & & & \multirow[b]{2}{*}{2 or $3(k)$} & \multirow[b]{2}{*}{$>25 \%(k)$} & HMA Cap $(g, h, i)$ & 8,9 & No Change & 1 to 3 years \\
\hline & & & & & $\begin{array}{c}\text { Replacement with Epoxy } \\
\text { Coated Rebar (ECR) Deck }\end{array}$ & 9 & 9 & $60+$ years \\
\hline \multicolumn{9}{|c|}{ 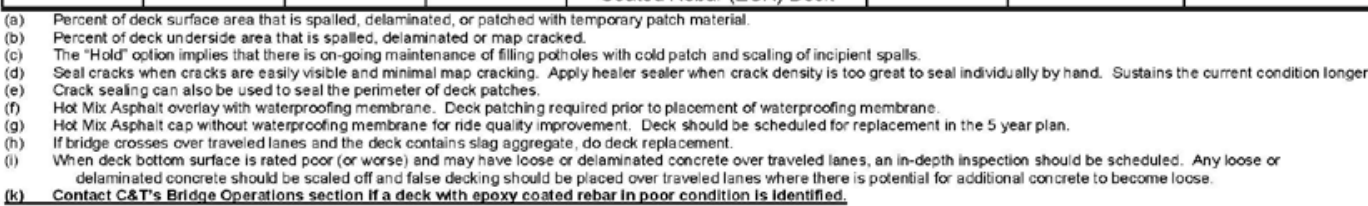 } \\
\hline
\end{tabular}

Figure 2.1: MDOT's RC bridge deck preservation matrix (MDOT 2011a) (See Appendix D) 
The deterioration of the sacrificial reinforced concrete deck is not the direct area of concern. Rather as the deck degrades the crucial load bearing structural elements, the superstructure and substructure, become more susceptible to deterioration or accelerated deterioration. By applying knowledge gained from experimental testing and utilizing emerging technologies, early signs of reinforced concrete deterioration would be identifiable and preventative steps could be taken to slow or reverse the process.

\subsection{Concrete Deterioration Mechanisms}

Concrete deck deterioration can be classified by the location of the defect. Two categories are utilized in classifying a defect, either a surface or subsurface, with one often leading to the manifestation of the other (Ahlborn et al. 2010). Several different deterioration mechanisms are investigated during field inspections, to quantify the condition of the reinforced concrete deck and the overall surface roughness, which contributes to the ride quality. Surface cracks, spalling, scaling, delaminations, voids and expansion joint issues are common methods of deterioration plaguing the National Bridge Inventory (NBI) infrastructure. All the discussed deterioration mechanisms draw on each other, resulting in a complex network of cause and effect. Figure 2.2 is simplified model of the deterioration cause and effect of concrete structures.

Each mechanism presents its own unique suite of challenges when being evaluated using LiDAR. Surface deteriorations can be directly quantified by way of LiDAR, but the subsurface deteriorations, delaminations and voids, cannot be directly quantified. However, these subsurface defects can be indirectly measured and correlated through surface indicators.

Commonly, reinforced concrete deck construction is a cast-in-place operation due to the economic advantages. However, the quality control of onsite construction causes vulnerability such as improper curing, environmental influence and human disruption. This lack of quality control increases the probability of accelerated or premature crack manifestation. 
Surface cracks can be classified into several different types, typically denoted by the crack configuration, width and cause. A crack is a linear fracture in concrete and can be categorized as either structural or non-structural. Structural cracking is the result of dead load and/or live load stresses exceeding the concrete capacity generating a substantial crack. Non-structural cracking is initially superficial and is caused by thermal expansion and shrinkage. Also non-structural cracking can be a function of the material design.

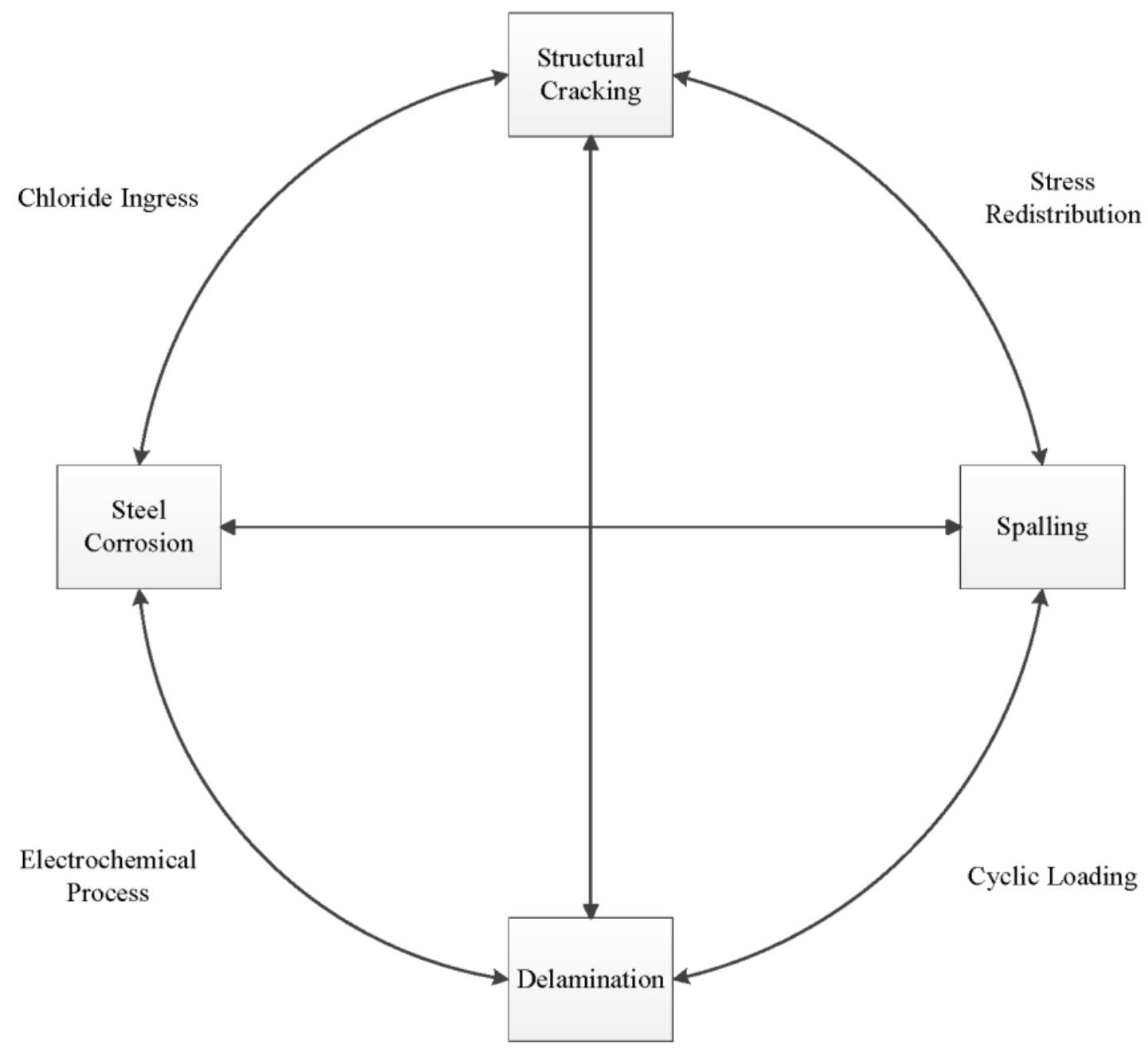

Figure 2.2: Reinforced concrete deterioration diagram 
The most common type of crack present in bridge decks are transverse cracks, which are often caused by restrained shrinkage and typically occur shortly after construction (Nowak et al. 2000). Transverse cracking propagates as a non-structural crack, but can cause localized stresses resulting in serious structural cracking. As the name implies transverse cracks develop transverse to the traffic flow. Common bridge construction techniques require the reinforcing steel closest to the concrete surface to be placed longitudinal to the traffic flow for flexural strength. With inadequate concrete cover over longitudinal reinforcing steel, chloride ingress is capable of initiating the steel corrosion process, developing tensile forces and causing longitudinally oriented cracking. The cracks resulting from the longitudinal steel corrosion process is referred to longitudinal cracking. Pattern or map cracking is associated with random, multi-directional cracking commonly resulting for inadequate freeze-thaw protection and the steel corrosion process. Examples of the three discussed crack varieties can be seen in Figure 2.3 (FHWA 2006). The traffic flow on Figure 2.3 is moving from left to right on the paper.

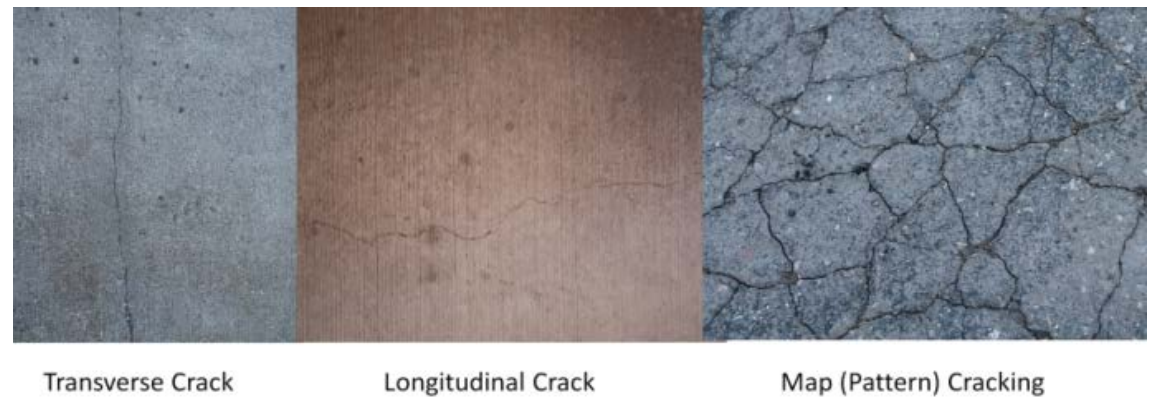

Figure 2.3: Classification of concrete cracks (FHWA 2006) (See Appendix D)

The pertinent measurement of a suspect crack is the width rather than the length, which can vary from a hairline opening to several inches. The reasoning behind width being the pertinent measurement is that the larger the crack width, the higher probability of chloride ingress resulting in steel corrosion. Current inspection standards indicate that a crack is considered structural when the crack width exceeds 1/16 inch $(1.59 \mathrm{~mm})$ as described in Table 2.1 (FHWA 2006). 
Table 2.1: Concrete crack width guidelines (FHWA 2006) (See Appendix D)

\begin{tabular}{|c|c|c|}
\hline Classification & English & Metric \\
\hline $\begin{array}{c}\text { Non-structural } \\
\text { Hairline }\end{array}$ & $<1 / 16$ in $(0.0625 \mathrm{in})$ & $<1.6 \mathrm{~mm}$ \\
\hline $\begin{array}{c}\text { Structural } \\
\text { Narrow }\end{array}$ & $1 / 16$ in to $1 / 8$ in $(0.0625$ in- $0.125 \mathrm{in})$ & $1.6 \mathrm{~mm}$ to $3.2 \mathrm{~mm}$ \\
\hline Medium & $1 / 8$ in to $3 / 16$ in $(0.125$ in- $0.1875 \mathrm{in})$ & $3.2 \mathrm{~mm}$ to $4.8 \mathrm{~mm}$ \\
\hline Wide & $>3 / 16$ in $(0.1875$ in) & $>4.8 \mathrm{~mm}$ \\
\hline
\end{tabular}

Another crucial measurement in assessing the condition of the bridge deck is crack density. A crack density classification is developed by measuring the cumulative lineal feet of cracks in a $100 \mathrm{~m}$ or $100 \mathrm{ft}$ long and $7.3 \mathrm{~m}$ or $24 \mathrm{ft}$ section of pavement, respectively. The Michigan Department of Transportation (MDOT), as most state agencies do, has grouped crack density for concrete pavement as listed in Table 2.2 for ease of classification. The crack density measurements allow for the inspectors to understand the severity of the distress when assessing pavement condition, which is similar to bridge decks (Reay et al. 1998). High crack density can be linked to material failure that typically requires replacement (FHWA 2006). For a trained bridge inspector, the presence of particular cracks and any associated crack density on a bridge deck is a good indication of an underlying subsurface deterioration mechanism.

However, being able to quickly locate and quantify surface cracking through the application of LiDAR, the inspector could generate an informed decision resulting in the appropriate course of action. The application of LiDAR can improve inspections by quickly locating and quantifying surface cracking and spalls. Rehabilitation and maintenance decisions could be enhanced due to the availability of subject rich data. 
Table 2.2: Concrete crack density guidelines (FHWA 2006) (See Appendix D)

\begin{tabular}{|c|c|c|}
\hline Density & $\begin{array}{c}\text { Linear Crack Length per } \\
\mathbf{1 0 0} \mathbf{~ m} \text { Pavement Section }\end{array}$ & $\begin{array}{c}\text { Linear Crack Length per } \\
\mathbf{1 0 0} \mathbf{f t} \text { Pavement Section }\end{array}$ \\
\hline Low & $<10 \mathrm{~m}$ & $<10 \mathrm{ft}$ \\
\hline Moderate & $10 \mathrm{~m}$ to $135 \mathrm{~m}$ & $10 \mathrm{ft}$ to $135 \mathrm{ft}$ \\
\hline High & $>135 \mathrm{~m}$ & $>135 \mathrm{ft}$ \\
\hline
\end{tabular}

An additional deterioration mechanism, delamination, is the direct result of the steel corrosion process. When the reinforcing steel corrodes, the cross sectional area increases due to the growth of corrosion products causing the bonded concrete surrounding the steel to crack and separate from the expanding metal. This subsurface deterioration mechanism is referred to as concrete delamination, but commonly this subsurface issue generates surface defects such as a spall. Theoretically, the delamination does not physically rise to the surface, but rather the now separated concrete material, which possesses little flexural capacity, breaks away under the applied cyclic vehicular loading. An example of a spall on a concrete bridge deck can be seen in Figure 2.4. Similar in appearance, scalling is a deterioration mechanism caused by material degradation resulting in material loss as seen in Figure 2.5. Current inspection practice denotes that any measurement of $1 / 4$ in $(6.35 \mathrm{~mm})$ in depth is the minimum consideration for spalled or scaled defects (FHWA 2006). 


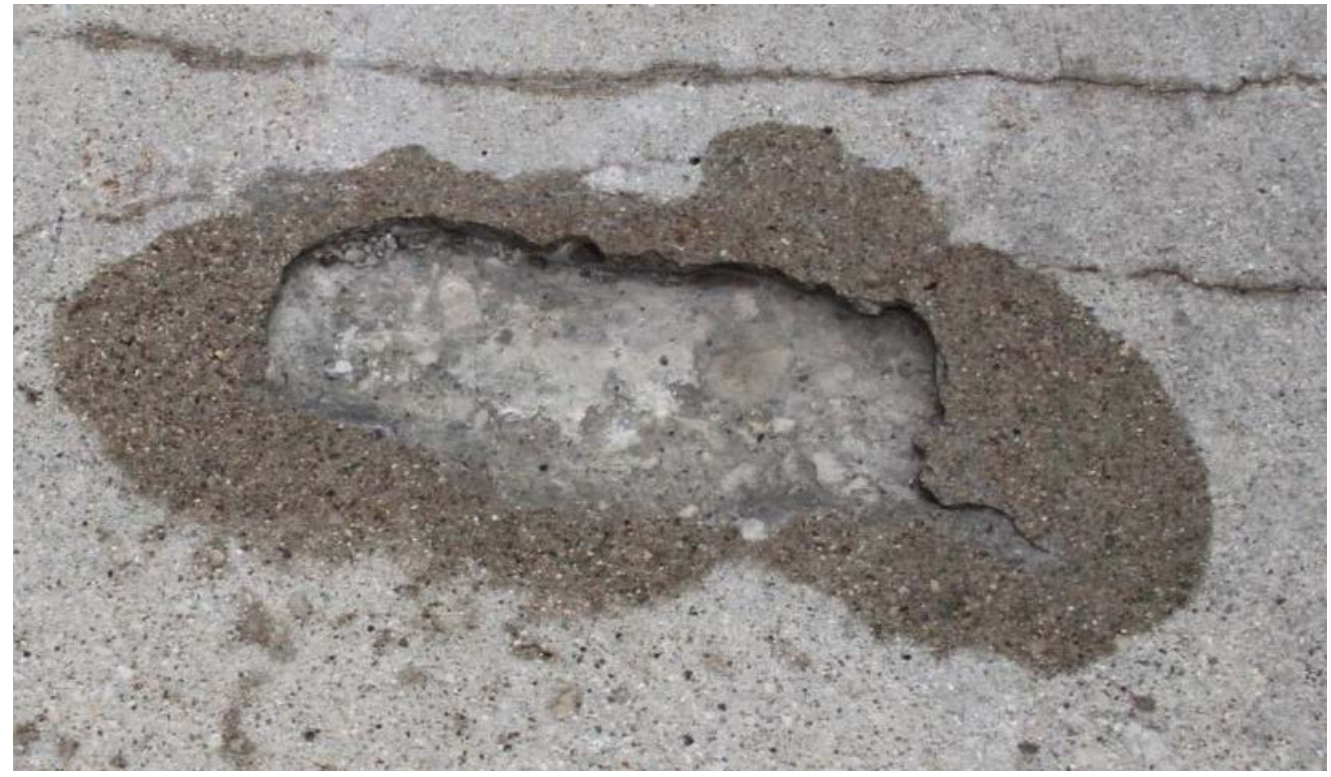

Figure 2.4: Example of concrete spalling (Courtesy of Renee Oats) (See Appendix D)

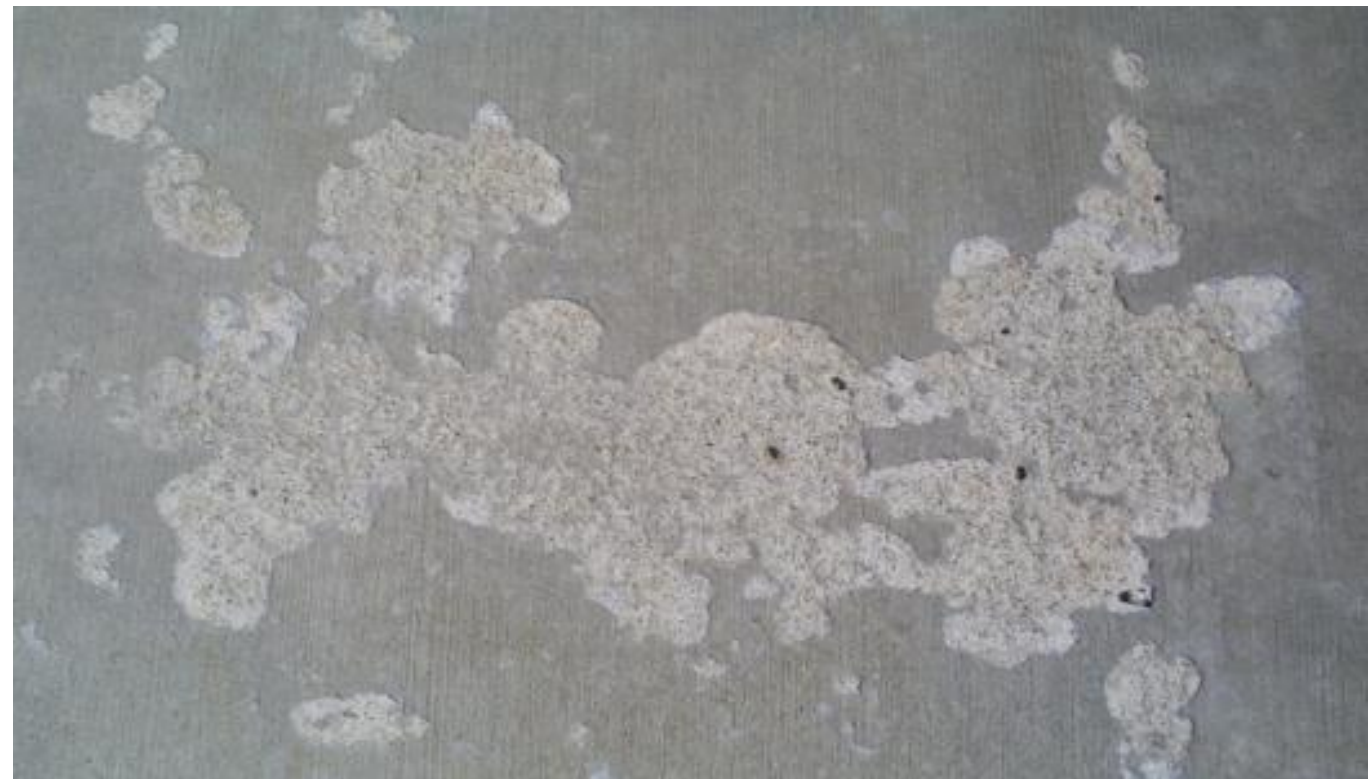

Figure 2.5: Example of concrete scaling (Courtesy of Renee Oats) (See Appendix D) 
Expansion joint issues are another primary concern when evaluating an in-service reinforced concrete bridge deck. The complex interaction shown in Figure 2.2 illustrates that the previously discussed deterioration mechanisms: cracking, spalling and scaling contribute to the expansion joint issue. Expansion joints allow for longitudinal expansion due to material thermal expansion. However, when cracks and spalls are allowed to propagate the loose material generated fills the expansion joint, inhibiting its function. By inhibiting the expansion joint, localized stresses due to material thermal expansion are generated inducing additional cracking and spalling in proximity to the expansion joint. Other common issues associated with expansion joints are torn or missing seals, armored plate damage and chemical leaching on the bottom of a joint (FHWA 2006).

The overall surface roughness is another issue considered when assessing a reinforced concrete bridge deck. Overall roughness is more commonly a user perception measure rather than a structural health indicator. Surface roughness in a pavement structure is commonly reported using a standardized roughness measurement referred to as the International Roughness Index (IRI) (Gillespie 1992). The IRI is a cumulative measurement of how much displacement is experienced within a single wheel path over a given mile. In essence the measurement indicates how much movement a vehicle's suspension will experience in a given mile. Typical values range from 0 inches per mile to 300 inches per mile on an extremely degraded road surface. However, due to the short spans associated with in-service bridges, separate IRIs are not available to the inspector, but rather incorporated into the IRI rating of the entire road network. The ASTM E192608 is the standard for determining IRI for roadway surfaces (ASTM 2008). No standard is available specifically for bridge decks.

\subsection{Development of Present Bridge Inspection Procedures}

Periodic bridge inspections are performed by state and local transportation agencies. To generate an educated determination of the safety and remaining service life of a bridge, periodic bridge inspections are critical. Maintenance, repair and rehabilitation measures are completed based on the results of the bridge inspection findings. Agencies perform 
many different types of inspections, including initial, routine, hands-on, fracture-critical, underwater, in-depth, scoping, damage, or special inspections (NCHRP 2007).

The governing standards for all bridge inspections in the United States jurisdiction are the National Bridge Inspection Standards (NBIS). The NBIS were developed after the Federal-Aid Highway Act of 1968 and required the Secretary of Transportation to establish a unifying bridge inspection document to ensure the safety of the traveling public traversing federally funded bridges. With the enactment of the Surface Transportation and Assistance Act of 1978, the NBIS was extended to cover all bridges greater than twenty feet linking public roads. The most recent adaptation of the NBIS was the result of the Surface Transportation and Uniform Relocation Assistance Act of 1987, which expanded the scope of the bridge inspection program to include special inspection procedures for fracture critical members and underwater inspection (FHWA 2004).

Under the Surface Transportation and Assistance Act of 1978 bridges publicly owned and greater than twenty feet in length must be inspected at least once every two years. This standard is a minimum and if a bridge is found to be in accelerated distress or serves as a key infrastructure link, the inspection frequency may be increased (FHWA 2004). The methods of data collection may vary from agency to agency, but must remain within the realm of acceptable testing standards under the NBIS. Visual inspection is the common method of inspection, even though it is the most subjective.

The Federal Highway Administration (FHWA) and the National Highway Institute (NHI) developed the Bridge Inspector’s Reference Manual (BIRM) in 2002. The BIRM details accepted bridge inspection programs, procedures and techniques (FHWA 2006). Additionally, inspection certification is required prior to entering into the bridge inspection career. This inspection certification is obtained upon completion of the NHI comprehensive training program. Continual learning of emerging inspection techniques is required to maintain one's bridge certification, which is accomplished by attending review courses offered by the FHWA and NHI. 


\subsection{Commonly Recognized Structural Elements}

The NBIS itemized key elements are required to be visually inspected to promote uniformity between bridge inspectors. Elements of interest in the NBIS evaluation are the deck, superstructure and substructure. The advancements made by the NBIS were to provide consistent standards for bridge safety inspections. These safety specific inspection standards generated limited quantifiable data to create performance-based decision support (FHWA 2004). As a result, the American Association of State Highway and Transportation Officials (AASHTO) developed the "Guide for Commonly Recognized (CoRe) Structural Elements” for condition assessment and rating of key bridge elements on a measureable performance metric scale. The condition state of an individual structural element is developed from narrative descriptions and quantities. Raw condition data that is collected can be transformed into useful bridge metrics allowing for the user to assess condition and allocate resources accordingly. This identification and evaluation process is further enhanced by including remote sensing technologies for inspection. By incorporating remote sensing technologies into routine bridge inspections, useful bridge metrics (e.g. percent delaminations, spalled area) would be directly captured removing the requirement for human interpretation.

For an element to be properly assessed, the entire element must be evaluated, but individual sections possessing larger quantities of deterioration are not given increased precedence. By adapting the current CoRe structural elements' guide for condition assessment, remote sensing technologies have the potential to accurately generate unbiased ratings. Condition state matrices are primarily used to rate the CoRe structural elements. In this research one element, a reinforced concrete deck was selected for evaluation. Table 2.3 shows the AASHTO CoRe structural element condition established condition state matrix for a reinforced concrete deck as an example (AASHTO 2011). 
Table 2.3: Reinforced concrete bridge deck condition state matrix (AASHTO 2011) (See Appendix D)

\begin{tabular}{|c|c|c|c|c|}
\hline Defect & $\begin{array}{c}\text { Condition } \\
\text { State } 1\end{array}$ & $\begin{array}{l}\text { Condition } \\
\text { State } 2\end{array}$ & $\begin{array}{l}\text { Condition } \\
\text { State } 3\end{array}$ & $\begin{array}{c}\text { Condition } \\
\text { State } 4\end{array}$ \\
\hline Cracking & None to hairline & $\begin{array}{l}\text { Narrow size or } \\
\text { density, or both }\end{array}$ & $\begin{array}{l}\text { Medium size or } \\
\text { density, or both }\end{array}$ & \multirow{4}{*}{$\begin{array}{l}\text { The condition is } \\
\text { beyond the } \\
\text { limits } \\
\text { established in } \\
\text { condition state } \\
\text { three(3), } \\
\text { warrants a } \\
\text { structural } \\
\text { review to } \\
\text { determine the } \\
\text { strength or } \\
\text { serviceability of } \\
\text { the element }\end{array}$} \\
\hline $\begin{array}{l}\text { Spalls/Delamination } \\
\text { /Patched Areas }\end{array}$ & None & $\begin{array}{l}\text { Moderate spall } \\
\text { or patch areas } \\
\text { that are sound }\end{array}$ & $\begin{array}{c}\text { Severe spall or } \\
\text { patched area } \\
\text { showing } \\
\text { distress }\end{array}$ & \\
\hline Efflorescence & None & $\begin{array}{l}\text { Moderate } \\
\text { without rust }\end{array}$ & $\begin{array}{l}\text { Severe with rust } \\
\text { staining }\end{array}$ & \\
\hline Load Capacity & No reduction & No reduction & No reduction & \\
\hline
\end{tabular}

\subsection{Concerns with Standard Bridge Inspection Procedures}

A visual inspection relies heavily on an inspector's experience, introducing variability into the equation due to inherent human nature. Inspection consistency is an area of growing concern. In regards to the quantity of scales and spalling size, inspectors commonly use crude measuring devices that can induce error into the inspection process. Cracks are typically located during a visual bridge inspection and then surrounding areas are sounded with an inspection hammer to confirm adequate subsurface bonding. Depth and crack gage measurements are rarely collected unless specified and as a result the probability of chloride ingress cannot be determined.

Both surface and subsurface defect detection are influenced by human interpretation. Knowing the dependence of human interpretation for distinguishing delaminated concrete from sound concrete and the high variability between inspectors, one can understand the issue. During detailed scoping inspections, delaminations are located through the inspector's interpretation of the concrete's acoustical response when struck by an inspection hammer or a chain drag is performed as depicted in Figure 2.6 and Figure 2.7, respectively. Both methods require the inspector to excite the concrete surface by either sticking the surface with a mallet or drag a chain across the surface. An area is 
considered to contain a delamination if the acoustic response changes pitch from low to high. The Nondestructive Evaluation Validation Center (NDEVC) reported that the delamination inspection method can result in variability ranging from 2\% to 69\%. (Scott et al. 2002). This range of variability was the result of 22 independent pairs of state bridge inspectors from various state agencies inspecting a bridge with known delaminations and a total percent delaminated area.

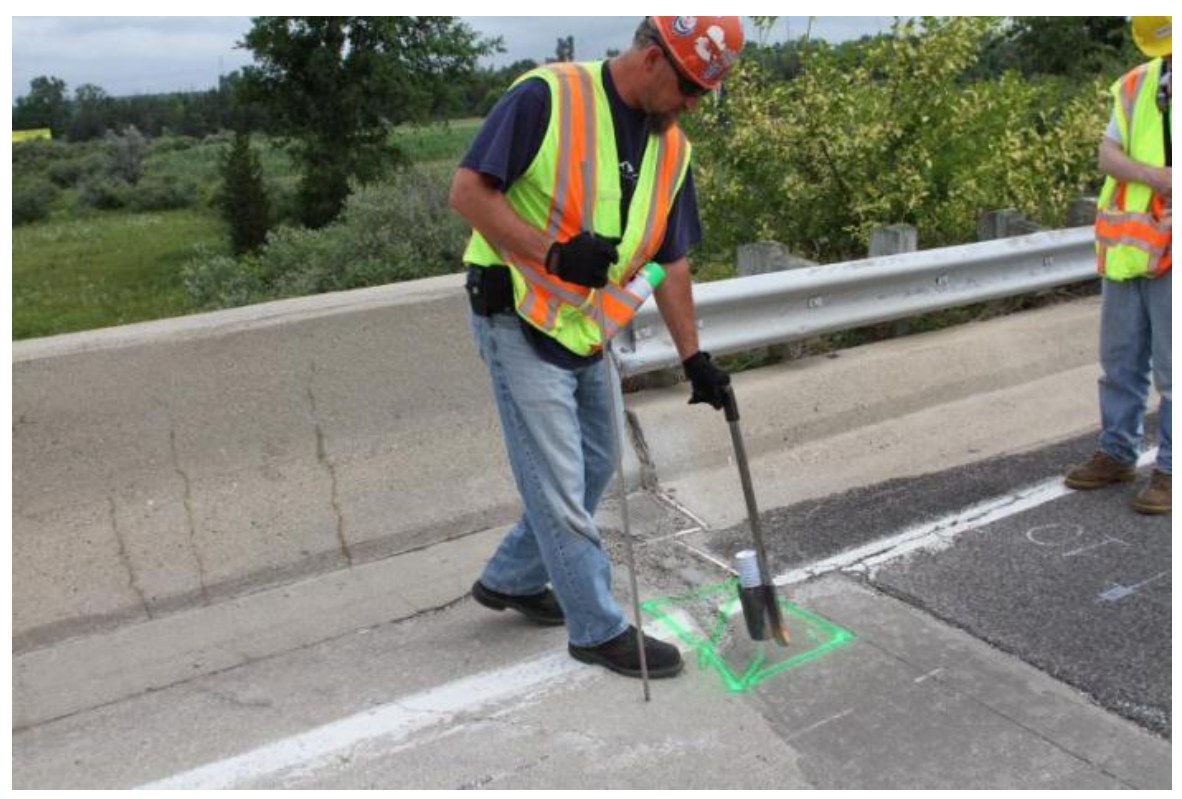

Figure 2.6: MDOT performed hammer sounding (Courtesy of Renee Oats) (See Appendix D) 


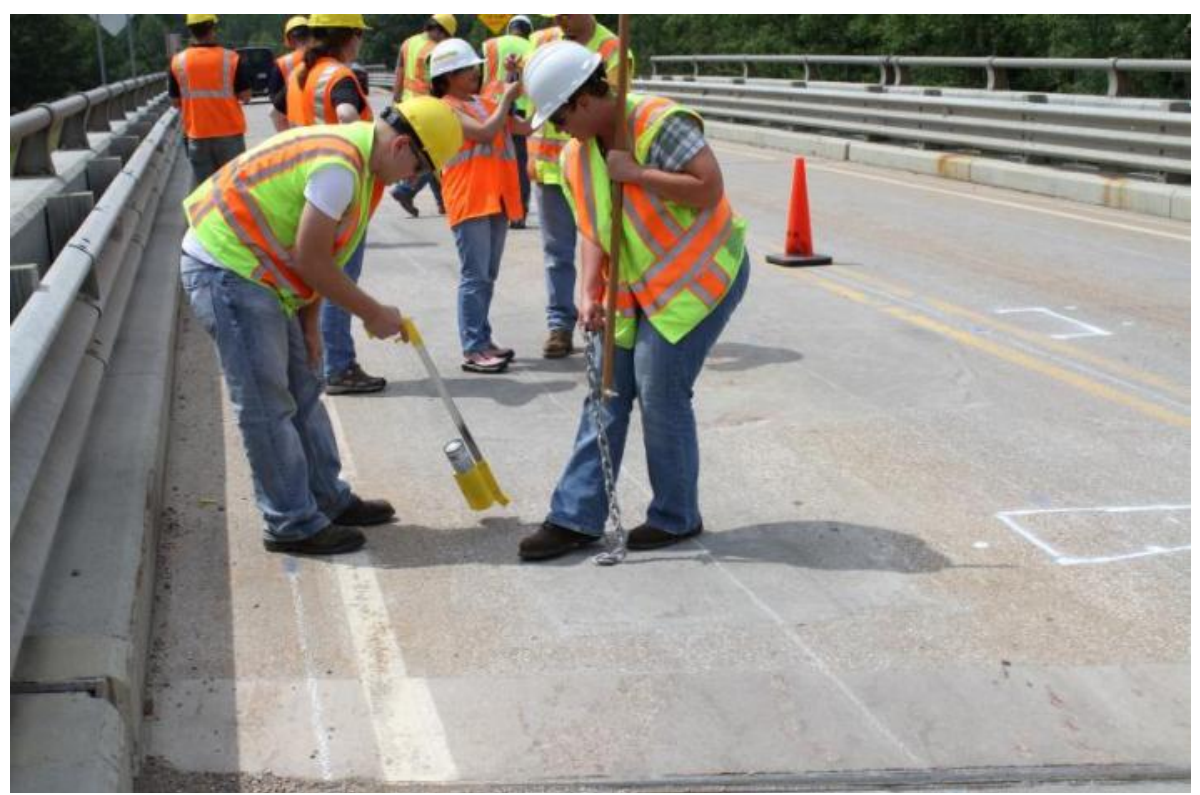

Figure 2.7: MDOT performed chain-drag testing (Courtesy of Renee Oats) (See Appendix D)

\subsection{Advanced Concrete Bridge Inspection Techniques}

Current inspection standards may not provide the necessary information to adequately allocate funding or conclusively determine the causes of deterioration. However, with the recent introduction of nondestructive testing (NDT) methods and retooling of inspection techniques previously used within other industries, the information gap is narrowing. Some of the well-known techniques are ground-penetrating radar, impactecho and infrared thermography. A majority of the developed NDTs are focused on subsurface defects. The reasoning is that currently subsurface defects are the most variable and difficult defects to sense. Additionally, there is reason to believe that by accurately identifying a surface defect, correlations could be developed to predict if there are subsurface anomalies present.

\subsubsection{Ground-penetrating Radar}

Ground-penetrating radar (GPR) is a form of radar acquisition characterized by relatively low electromagnetic frequencies, with center frequencies as low as $100 \mathrm{MHz}$, but usually no lower than $500 \mathrm{MHz}$. GPR commonly has a wide bandwidth allowing for maximum 
depth penetration while having the sensitivity to detect embedded features (Ahlborn et al. 2010). The transmitted radar waves are passed through the concrete deck and a detector measures the reflected energy. The reflected energy is then passed through a computer algorithm producing images. To decipher the images and identify if deterioration is present, a skilled operator is required. GPR has been shown to detect both surface and subsurface defects such as cracks, delaminations and steel corrosion (Gastineau et al. 2009). Tremendous strides have been taken in the last decade to produce a viable, practical and cost effective GPR system. The introduction of air-coupled, vehicle mounted systems allows for faster data collection and limited traffic disruption.

\subsubsection{Impact-echo}

Impact-echo is similar to the hammer sound or chain drag bridge inspection techniques for detecting subsurface defects through acoustical responses. The method was developed in 1983 as a result of a shift in the National Bureau of Standards' research on NDT detection of internal defects within concrete structures. Impact-echo was based on the knowledge of stress wave propagation in a solid, which is directly affected by the mechanical properties of the material. The theory is that if an internal defect were present in the concrete test media, the mechanical properties changes resulting in varying stress wave propagation. Typical designs revolve around a four wheeled device, which has a built-in striker to produce the acoustic wave and a microphone to measure the reflected response, Figure 2.8 . 


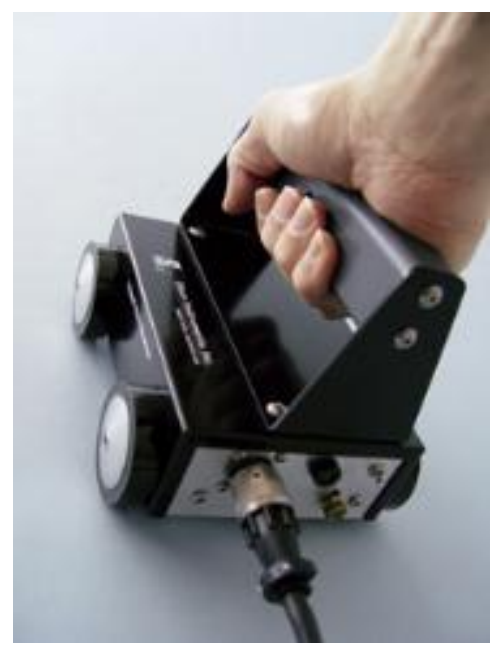

Figure 2.8: Hand held impact-echo equipment

By deviating from the traditional inspection techniques, the National Bureau of Standards sought to mitigate the need for human interpretation. The human interpretation is substituted by a computer algorithm that processes measured responses and determines not only if there is a defect present, but also at what depth. Determination of the depth of the defect can be determined if the measured response indicates a depth in the slab less than the depth of the actual slab (Gastineau et al. 2009). Current applications of this technology are to detect subsurface defects such as delaminations, voids and cracks. Rate of data collection is currently the primary concern hindering this technology, because many test locations must require traffic disruption. Vehicle mounted instruments are presently under investigation allowing for an increased data collection rate.

\subsubsection{Infrared Thermography}

Infrared thermography is based on the radiant temperature measurement of concrete media by a thermal infrared camera. This method takes advantage of concrete's thermal properties in which the radiant temperature will uniformly increase or decrease except in locations that have subsurface anomalies (Ahlborn et al. 2010). These subsurface defects will demonstrate a higher rate of radiant temperature change than the surrounding concrete, producing "hot spots" within the thermal infrared image, as shown in Figure 2.9. 
Issues with thermal imaging technology are mainly focused around environmental conditions and external containments. The requirement to induce a radiant temperature change in the concrete has developed a reliance on passive solar illumination. The use of solar illumination creates an enormous dependence on environmental conditions. However, the use of artificial illumination or cooling can relieve the dependence on environmental conditions, but requires a tremendous amount of energy to generate a significant radiant temperature contrast. External containments can also affect the method's reliability; paint, oil spots, standing water or other debris can affect the results generating false positives. In regards to subsurface defect detection, data collected from infrared thermography is a relatively simple analysis and thus makes the potential of this technology very useful. 


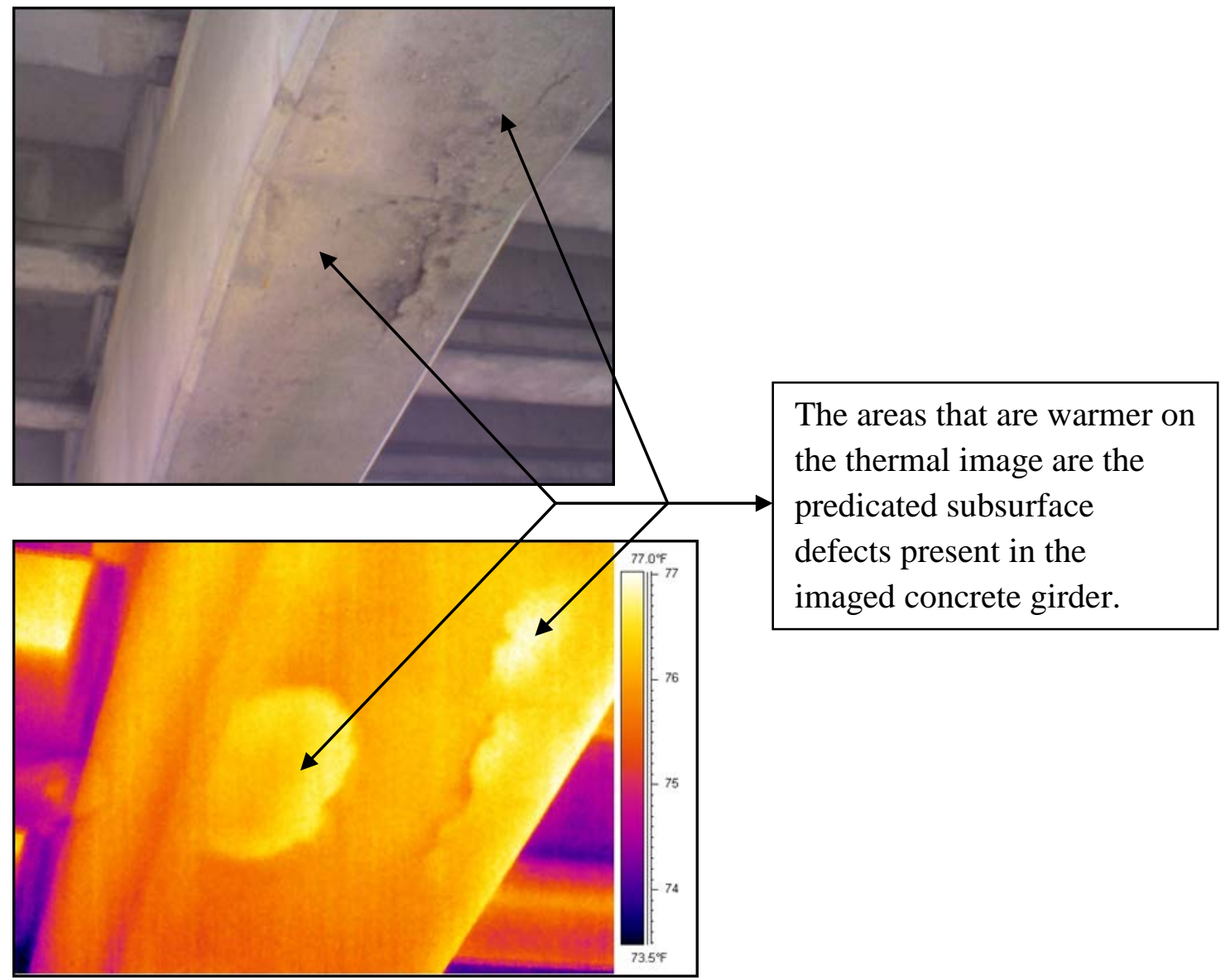

Figure 2.9: Optical and thermal image highlighting observable surface defect (Courtesy of Khatereh Vaghefi) (See Appendix D)

\subsection{Fundamentals of LiDAR}

Light Detection and Ranging (LiDAR), also referred to as Laser-Assisted Data and Readout (LADAR), is an optical remote sensing technology that can gather information, such as distance to, reflectivity or other properties, of a target object. This system dates back millions of years, when SONAR (SOund Navigation and Ranging) the oldest known variation of modern LiDAR systems evolved naturally as a guidance system used by bats (Schnitzler and Moss). Bats use SONAR by emitting sound waves in the form of short 'chirps' from their noses. The bats hear the echo through their ears that act like two antennae, which provide a three-dimensional rendering of their surroundings. LiDAR, SONAR and RADAR are all similar in theory; however, each detection system uses a different form of energy to emit a signal resulting in different applications. 
A basic LiDAR system consists of a transmitter (radiation source), a receiver (detector) as well as a control and data acquisition system (Chu 2011). Figure 2.10 shows the basic LiDAR components and their interactions. Typically, LiDAR employs ultraviolet, visible, or near infrared light to image objects by eradiating the object with a light energy source. LiDAR technology has numerous applications, to list a few; geomatics, archaeology, geography, geology, geomorphology, seismology, forestry, remote sensing and atmospheric physics. Additionally, adaptations to the technology have allowed for 'airborne laser swath mapping' (ALSM), 'laser altimetry' and LiDAR Contour Mapping by utilizing airborne platforms.

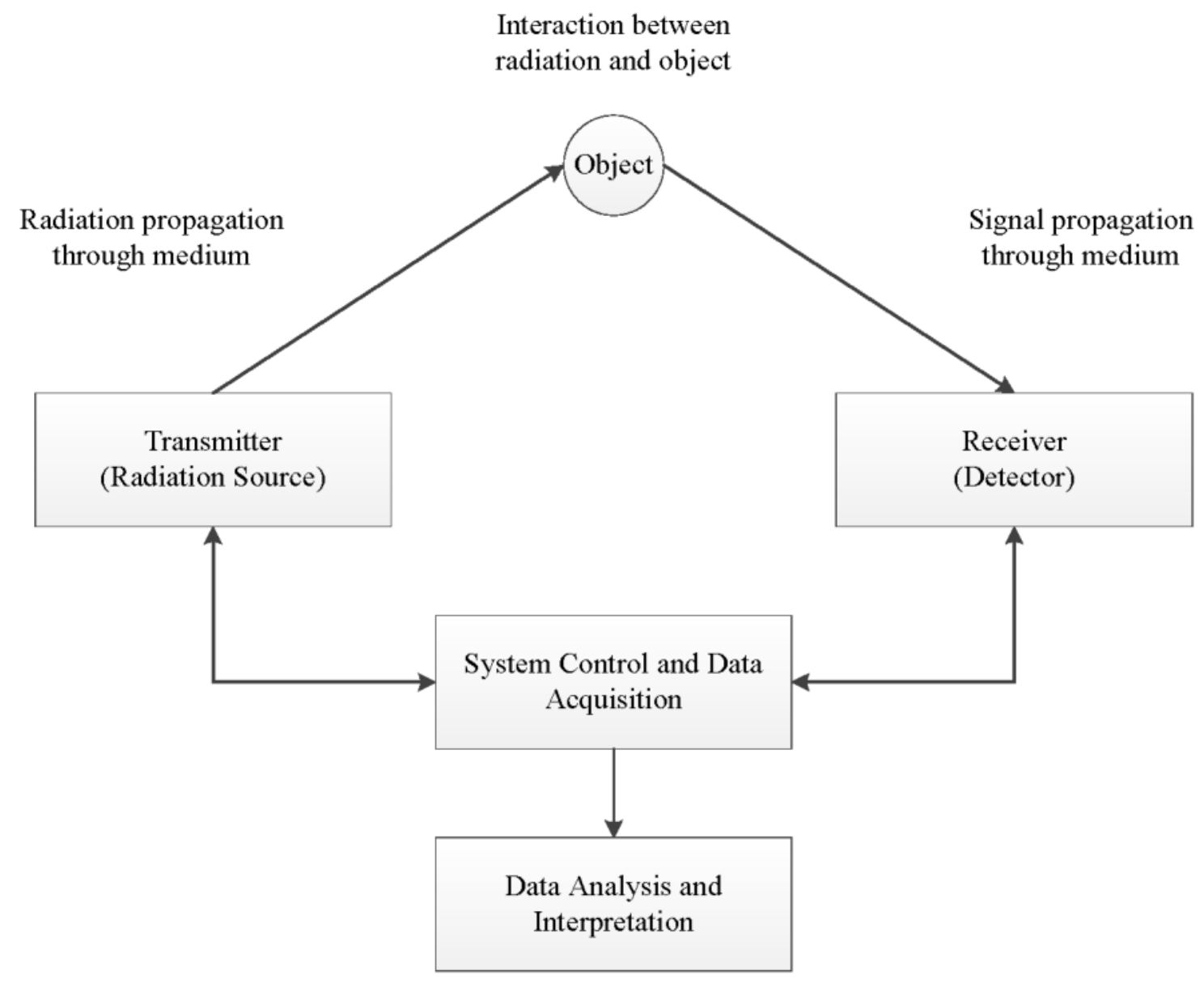

Figure 2.10: Basic LiDAR function flow chart 
A LiDAR transmitter is required to provide a radiation source, laser pulses that have a known wavelength, frequency accuracy, bandwidth, pulse duration, pulse energy, repetition rate and divergence angle. Normally, a transmitter consists of four systems including laser bundles, collimating optics, diagnostic equipment and a wave length control system. Two laser classes are employed in modern LiDAR application, which are nanosecond ( $n s)$ pulsed lasers (time-of-flight scanners) and continuous wave $(\mathrm{cw})$ lasers (phase-shift scanners). As the names imply the fundamental difference between the two laser classes is one laser pulses, while the other is a continuous emission of radiation. Most modern LiDAR systems employ a ns pulsed laser, primarily due to the fact that the pulsing action allows for the light emitting diode to remain cool allowing the output magnitude to be increased without shortening the diode’s lifespan. For LiDAR systems with spectral analysis capabilities, the transmitter is usually the most critical and challenging component. The performance and accuracy of a LiDAR system is primarily driven by the properties associated with its respective transmitter (Chu 2011).

A LiDAR's receiver or detection component identifies and collects reflected photon signals from the illuminated object while minimizing background noise. The receiver is comprised of telescopes, filters, collimating optics, photon detectors and discriminators. One key component to highlight is the filter, because the bandwidth of the filter determines whether the receiver can spectrally distinguish the reflected photons.

Serving as the intermediary between the transmitter and receiver is the data acquisition and control system. This device records the reflected data and corresponding time-offlight information while providing system control and coordination. The data acquisition and control system consists of a multi-channel scalar, which contains a very precise clock for data tagging, a discriminator, computer and respective software. As LiDAR systems advance, the data acquisition and control system becomes more important, because the data collection rate and abundance of points continues to increase.

There are two common component configurations used, bistatic and monostatic, depending on the application. A LiDAR system with a bistatic configuration is ordinarily 
used for applications requiring considerable spatial resolution over a large area. This degree of spatial resolution is achieved by separating the transmitter and receiver by a considerable margin. For a monostatic configuration, both the transmitter and receiver are located in the same place. By placing both the transmitter and receiver in the same location, the user has in effect created a single-ended system. This single-ended system allows for precise determination of range and reflectivity. To achieve a high magnitude of precision while maintaining a sufficient collection speed, a monostatic LiDAR system utilizing a ns pulsed laser is preferred. The fundamental differences between the bistatic and monostatic LiDAR configurations are shown in Figure 2.11.

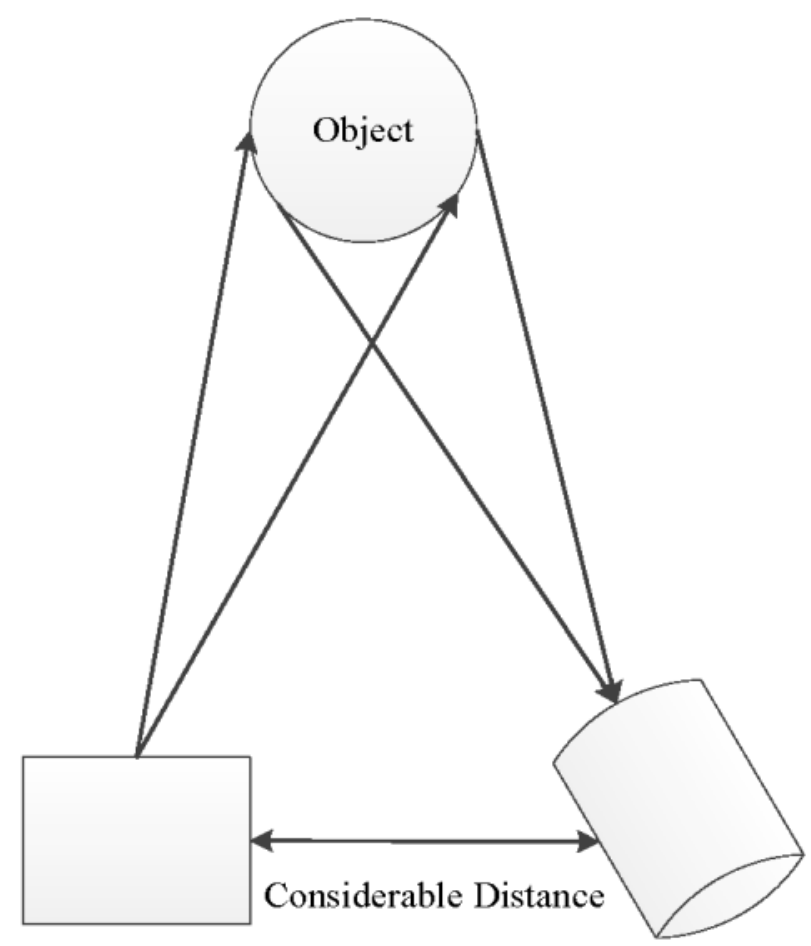

Transmitter
Receiver

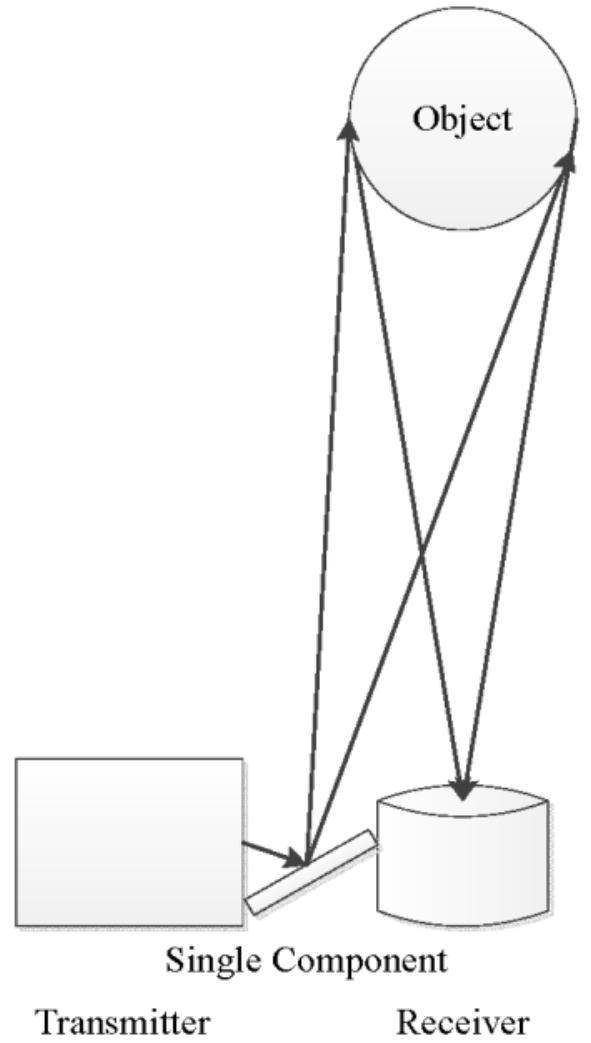

Monostatic Configuration

Bistatic Configuration

Figure 2.11: Bistatic vs. monostatic LiDAR configurations 
A monostatic LiDAR configuration is organized in either a coaxial or biaxial arrangement. In a monostatic, coaxial arrangement, the axis of the ns pulsed laser beam is coincident with the receiver optic axis (Chu 2011). This arrangement allows for the detection of near-field backscattered radiation or detection of close objects. However, over saturation of the receiver optics is common for coaxial systems, so gating the receiver optics, implementation of a fast shutter or chopper is often required. The biaxial arrangement has a predetermined range in which the ns pulsed laser beam will not enter the field-of-view of the receiver optics until meet, helping to avoid the previously discussed backscattered radiation over saturation.

\subsection{LiDAR Operating Parameters}

Compared to traditional surveying equipment, a LiDAR system can collect millions of data points in a single pass of a suspect object allowing for a detailed analysis to be completed. An issue arises when the object has many faces producing "shadows" or “blind spots” in the instrumentation's field-of-view. With common monostatic or biaxial time-of-flight terrestrial LiDAR systems, the radiation source and receiver are in the same location and any surface eradiated by the transmitted energy, which reflects the residual energy directly back to the receiver will be identified. Any surface with limited reflectivity or refracting properties results in limited energy return to the receiver and causes false "blind spots” represented as empty space. The LiDAR's line-of-sight issue can be resolved by repositioning the device numerous times allowing for the hidden surfaces of the complex object to be revealed. By combining the multiple point clouds collected from each reposition a full three-dimensional rendering of the object after the multiple collection points are fused together.

LiDAR works by radiating an object and detecting the reflected radiation to accurately determine the distance to the reflected object. This method of distance measurement is similar to a laser rangefinder, but rather than making a single measurement, LiDAR units utilize rotating mirrors (or the entire unit rotated allowing for the collection of millions of measurements over a scene) (CFLHA 2011). Methods of radiation and data collection 
differ between $n s$ pulsed laser (time-of-flight) and $c w$ laser (phase-shift), but the distance calculations are similar.

For time-of-flight (TOF) laser scanners utilize a sensor to measure the TOF for the optical radiation pulse to travel to and from the reflected surface. A simple calculation is then automatically performed within the data acquisition and control system to determine the object's distance from the receiver. The calculation for determination of the travel distance of a pulse is as follows:

\section{Distance $=($ Speed of Light $\mathrm{x}$ Time-of-Flight $) / 2$}

TOF scanners have multiple modulation frequencies that are utilized to increase the measurement accuracy. Additionally, TOF scanners can be used to measure different data sets bounded by the emitted radiation's return time. This feature is primarily used in forestry applications allowing for the generation of both a canopy profile with the "first return" and a ground surface profile with the "last return".

In phase-shift units, a continuous laser beam with sinusoidally modulated optical power is projected from the transmitter and reflected off the suspect object. The reflected radiation wave is then sensed by the receiver and compared to the original emitted radiation to determine the present phase shift within the acquired data. Once the phase shift is determined, the TOF is then automatically calculated using Equation 2:

$$
\text { Time-of-Flight }=\text { Phase Shift } /(2 \pi \times \text { Modulation Frequency })
$$

The distance of the illuminated object is then calculated by substituting the determined TOF from Equation 2 into Equation 1.

A single $n s$ pulse signal received and measured can only determine the perpendicular distance from the receiver face to the illuminated object. To orientate the object in three- 
dimensional space the LiDAR unit repeats the scanning process millions of time per second referencing each received and measured pulse to the previous pulse. From the distance and the relative orientation of the laser pulse, the xyz coordinates associated with each measured pulse can be determined in relative space (Chu 2011). The outputted information containing the xyz coordinates and associated intensity values for these millions of sensed data points is referred to as the "point cloud”. From this "point cloud", a user can access and visually display the collected data in virtual space. Software can be used to manipulate and extract features of interest present within the rendering.

\subsection{Fixed Terrestrial LiDAR Performance Evaluation}

Original applications of LiDAR only required high relative precision (relative dimensions within the registered point cloud), making the system not applicable to infrastructure application. To apply LiDAR to the infrastructure sector, the technology needed good relative precision and high absolute accuracy (robust geo-referencing). Performance testing and evaluation of several commercially available LiDAR units were completed focusing on pertinent attributes necessary for the desired application.

Generally, LiDAR's capabilities, such as accuracy and object detection, are highly dependent on target range, object reflectivity, and angle of incidence to the reflective surface (Hiremagalur et al. 2007). Each LiDAR manufacturer has different methods to determine specifications such as accuracy terms and often their own trademark terminology. Limited standard testing protocol has been implemented making it impossible to compare LiDAR units solely from the manufacturer's specifications. Thus, performance evaluations of fixed terrestrial 3D laser scanning systems for highway application are necessary.

Data presented in the fixed terrestrial LiDAR performance evaluation section was generated by the Advanced Highway Maintenance and Construction Technology (AHMCT) Research Center at the University of California, Davis. The AHMCT conducted testing and performance evaluations of fixed terrestrial 3D laser scanning systems for highway applications. The three LiDAR units evaluated by the AHMCT 
were a Leica ScanStation C10, a Trimble GX and an Optech ILRIS-3D. The control test evaluated the individual LiDAR systems’ performance in an outdoor pavement environment with maximum repeatability for the available testing conditions. Test fixtures were positioned on tripods on the side of a selected asphalt section and were scanned from one stationary point. Test fixtures were designed to test each scanner's range precision, target recognition precision, resolution and the effects of target reflectivity and laser incidence angle (Hiremagalur et al. 2007). The five control test fixtures shown in Figure 2.12.

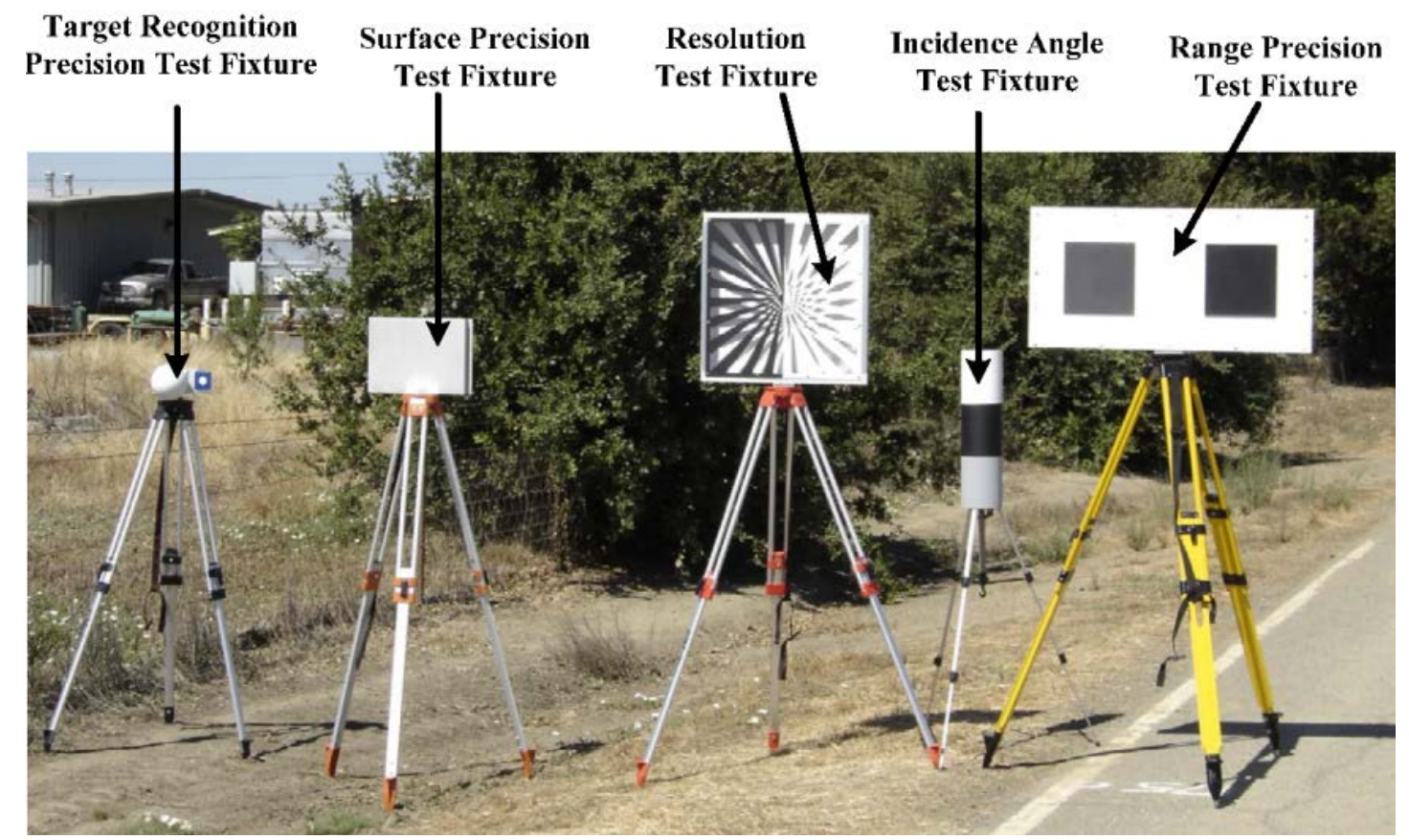

Figure 2.12: Control test fixtures (Hiremagalur et al. 2007) (See Appendix D)

The range precision test fixture was comprised of two anodized flat aluminum plates, one with a dull gray finished (reflectivity $\sim 40 \%$ ) and one with a flat black color finish (reflectivity $\sim 10 \%$ ). The two flat aluminum plates were mounted on a flat $102 \mathrm{x} 51 \mathrm{~cm}$ (40 x 20 in) aluminum plate painted flat white (reflectivity 80\%). The scanners and range precision test fixture were positioned in the same vertical plane creating an angle of incidence of zero degrees. Point cloud spacing varied from 3 to $10 \mathrm{~mm}$ depending on the internal scanner components and the software utilized. The collected data were analyzed 
for root-mean-square error (RMSE) of range precision a with 95\% confidence interval. Table 2.4 shows the results from the range precision test fixture evaluation. The results revealed that as the target distance increased from 50 to $100 \mathrm{~m}$ the RMSE gradually increased, producing lower confidence in the equipment ability to resolve features over distance (Hiremagalur et al. 2007).

Table 2.4: RMSE (mm) of range precision at $95 \%$ confidence interval (Hiremagalur et al. 2007) (See Appendix D)

\begin{tabular}{|c|c|c|c|c|c|}
\hline \multicolumn{2}{|c|}{} & \multicolumn{5}{|c|}{ Range precision, 95\% RMSE (mm) } \\
\hline \multirow{3}{*}{ Manufacturer } & Range & $25 \mathrm{~m}$ & $50 \mathrm{~m}$ & $75 \mathrm{~m}$ & $100 \mathrm{~m}$ \\
\cline { 2 - 6 } & Color & \multicolumn{4}{|c|}{} \\
\hline \multirow{3}{*}{$\begin{array}{c}\text { Leica } \\
\text { ScanStation }\end{array}$} & White & 4.65 & 3.23 & 3.23 & 4.78 \\
\cline { 2 - 6 } & Grey & 4.72 & 4.31 & 4.68 & 5.49 \\
\cline { 2 - 6 } & Black & 4.72 & 3.45 & 3.65 & 7.08 \\
\hline \multirow{3}{*}{\begin{tabular}{c} 
Trimble GX \\
\cline { 2 - 6 }
\end{tabular}} & White & 2.10 & 1.65 & 2.20 & 1.84 \\
\cline { 2 - 6 } & Grey & 2.98 & 4.82 & 4.92 & 7.74 \\
\hline \multirow{2}{*}{\begin{tabular}{c} 
Optech \\
\cline { 2 - 6 }
\end{tabular}} & Black & 3.00 & 4.82 & 7.80 & 11.70 \\
\cline { 2 - 6 } & Grey & 13.30 & 14.31 & 16.48 & 21.76 \\
\cline { 2 - 6 } & Black & 13.07 & 14.07 & 18.93 & 18.37 \\
\hline
\end{tabular}

The target recognition precision fixture was a $15 \mathrm{~cm}$ (6 in) diameter spherical target with a vendor-specific planar registration target mounted on a linear stage driven by a highprecision lead screw that provided accurate and repeatable millimeter-level translation (Hiremagalur et al. 2007). Both targets were on the same horizontal plane as the scanner and the recognition target's face was perpendicular to the scanner. Scans were collected for the Trimble and Leica units at 25 and $75 \mathrm{~m}$. After completion of the range precision evaluation, AHMCT found the Optech scanner inadequate for the target recognition precision. The results showed that as the test range increased, the target recognition precision of both the Trimble and Leica scanners shifted from an underestimation to an 
overestimation. With a single translation of the target recognition precision fixture, the maximum underestimation was $0.75 \mathrm{~mm}$ at a range of $25 \mathrm{~m}$ and the largest overestimation was $0.80 \mathrm{~mm}$ at a range of $75 \mathrm{~m}$. The target recognition precision was determined to be a linear function with decreasing precision as the range increased. The location of greatest precision was not the closest range tested, but rather a range of $50 \mathrm{~m}$ \pm 5 m depending on the terrestrial LiDAR unit assessed.

The coverage and incidence angles were determined for the three scanner units by evaluating a $15 \mathrm{~cm}$ (6 in) diameter cylinder. For a visual representation of the angle of incidence and coverage angle refer to Figure 2.13. The test fixture was painted white, flat grey and flat black to assess not only the limiting angle of incidence, but also how the coverage angle varied with different reflectivity. When considering the Light Reflectance Value (LRV), which is the total quantity of visible and useable light reflected by a surface in all directions and at all wavelengths when illuminated by a light source, the white paint should theoretically have the largest potential for reflecting light. A LRV chart is provided in Figure 2.14. The results from the testing demonstrated this known LRV characteristic by showing that the white, gray then black paint produced the largest to smallest coverage angle, respectively.

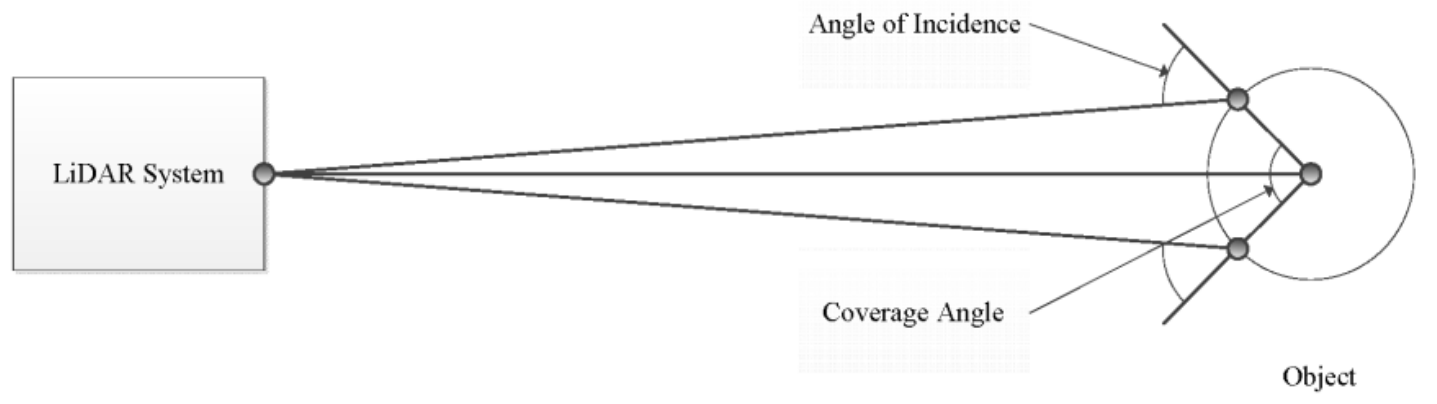

Figure 2.13: Illustration of incidence and coverage angles

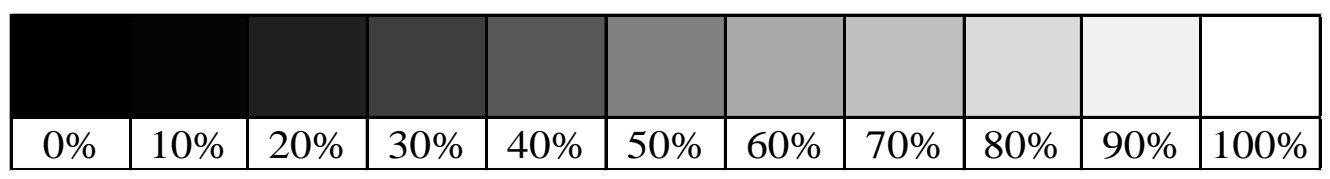

Figure 2.14: Light reflectance spectrum 
Theoretically, the maximum angle of incidence for a given scanner should not exceed $90^{\circ}$ and the maximum coverage angle should be less than $180^{\circ}$. The results found showed that due to the scale of the testing fixture a phenomenon referred to as the "edge effect" and the methods of calculating coverage angle generated maximum coverage angle equal to $180^{\circ}$ (Hiremagalur et al. 2007). However, the results allowed for a comparison between the scanning units and the color reflectivity from the data collected at 25, 50, 75 and $100 \mathrm{~m}$. The Leica unit produced a coverage angle of $180^{\circ}$ for both the white and gray paints at every scanning range, which was considered optimal for this experiment. Both the Leica and Trimble scanners demonstrated a lower coverage angle for the identical colors and the black paint resulted in the least desirable coverage angle for all units.

The definition of resolution for LiDAR systems and other remote sensing technology is currently a subject of debate within NIST and the ASTM International E57 Committee on 3D Imaging Systems. However, the AHMCT Research Center described resolution as the ability of the laser scanner to detect, differentiate and record 3D details or features of an object within the scanner's range and field-of-view (Hiremagalur et al. 2007). This definition of resolution diverges from the accepted description of image resolution, which uses the number of pixels to evaluate the quality of the image, because laser scanners do not produced images consisting of pixels.

A firm understanding of the internal components of the LiDAR unit is critical to understand the potential resolution of the scanner. Laser scanner "Resolution" depends on laser spot size and the smallest angle increment between two consecutive point measurements (Hiremagalur et al. 2007). For both $n s$ pulsed and $c w$ lasers the light emitting diode produces a circular, collimated beam. This collinear property results in the laser beam's photons to spread slowly apart as it propagates away from the emission source, generating data fall off the further the subject target is from the unit. To overcome divergence issues during data collection, focused high energy laser beams with smaller spot sizes are utilized. This smaller laser spot size results in higher energy per unit area at the target, leading to higher probability of defect detection. 
The AHMCT Research Center performed testing to visually illustrate and compare the loss of resolution over distance. The tests were performed at four ranges 25, 50, 75 and $100 \mathrm{~m}$. The test fixture consisted of a $61 \mathrm{~cm}$ (24 in) square box with a machined front panel and tapered slots decreasing from about $6.4 \mathrm{~cm}$ (2.5 in) wide at the periphery to about $0.25 \mathrm{~cm}$ ( $0.1 \mathrm{in})$ at the center. Contrasting paints were used to demonstrate reflectivity influence on resolution by painting half the front panel with white flat paint, and the other half with black flat paint, in addition the rear panel was also painted flat white. The target was mounted on a tripod, facing perpendicular to the laser scanner and at the same elevation, such producing an angle of incidence of near $0^{\circ}$. Figure 2.15 is provided to show a visual representation of the resolution's dependence on reflectivity and range. Additionally, a finite rectangular band width across the front panel was isolated to show the resolution variations from each LiDAR unit. The results from that post-processing sample are shown in Table 2.5. 


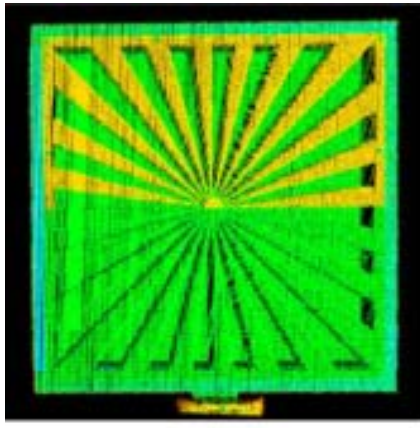

Entire laser point cloud of test fixture

\section{Leica $25 \mathrm{~m}$}

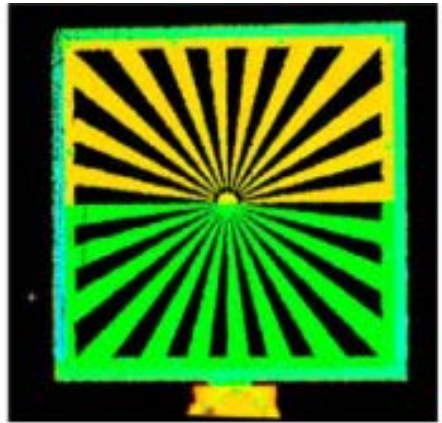

Laser return from front panel

Laser returu from rear panel

\section{Isometric view with front surface cropped}
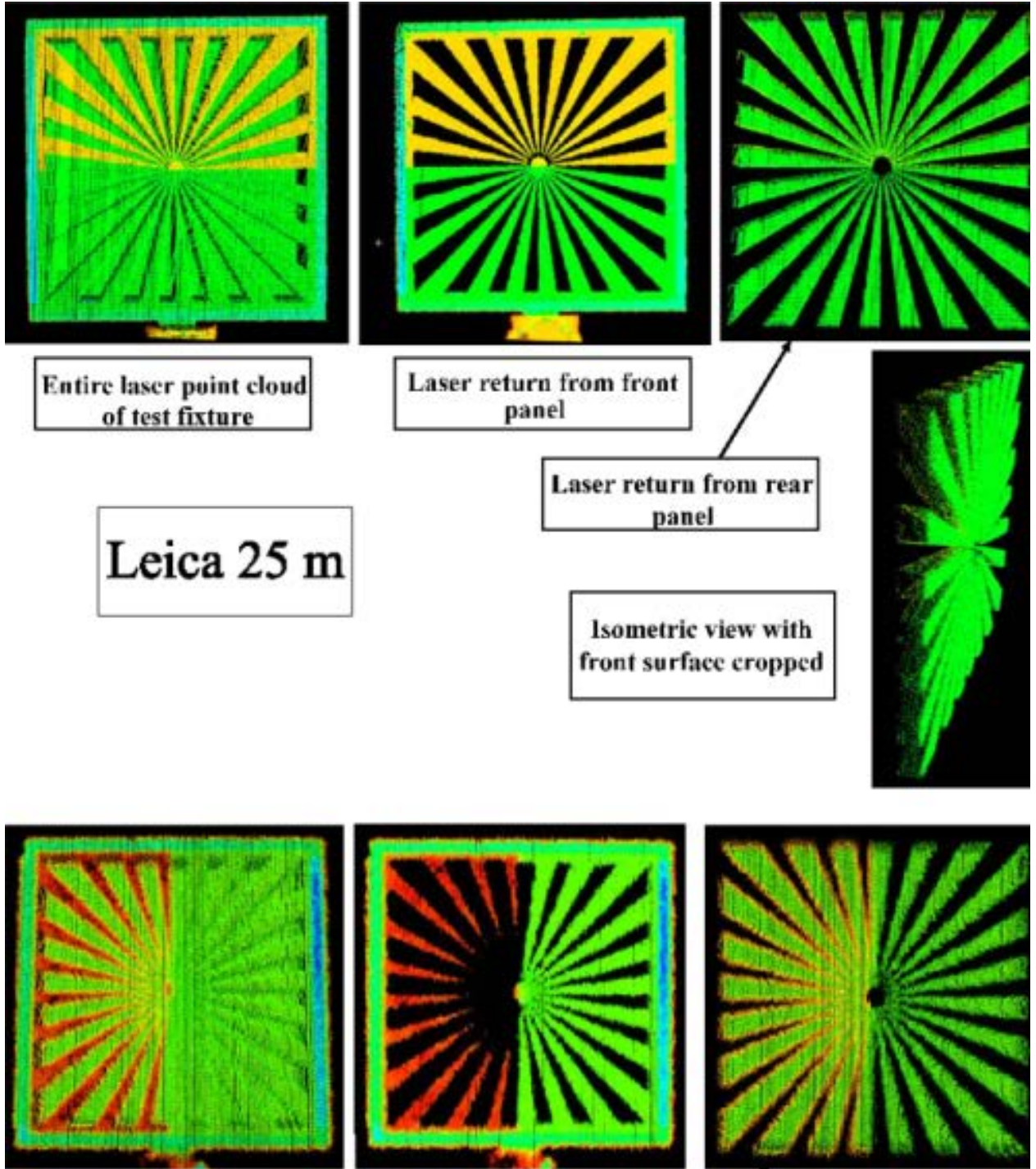

Entire laser point cloud of test fixture
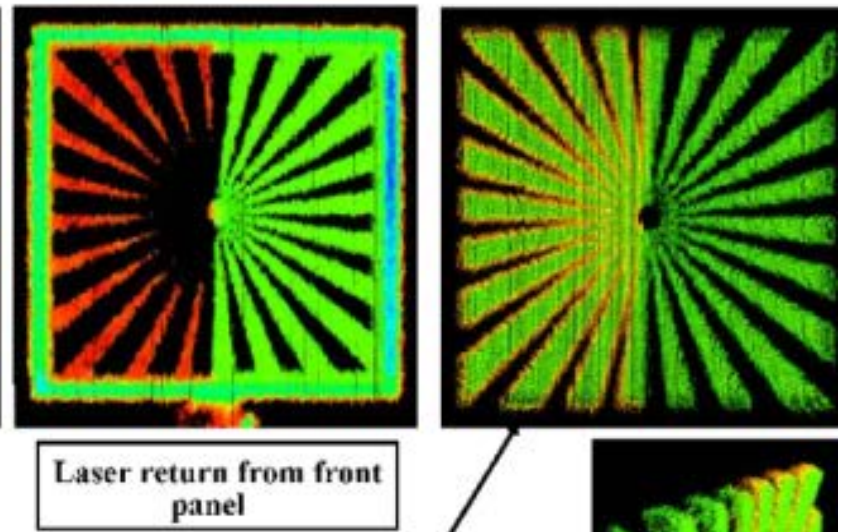

Laser return from rear panel

\section{Leica $100 \mathrm{~m}$}

Isometric view with front surface cropped

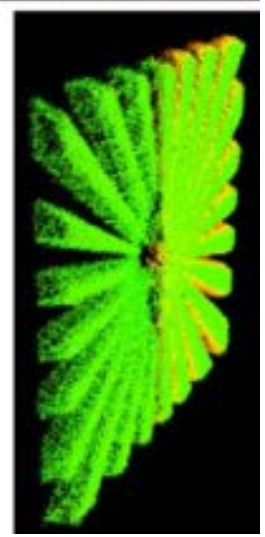

Figure 2.15: Example of laser point cloud resolution test fixture (Leica) at $25 \mathrm{~m}$ and 100 m (Hiremagalur et al. 2007) (See Appendix D) 
Table 2.5: Laser point cloud data of central cross-section (Hiremagalur et al. 2007) (See Appendix D)

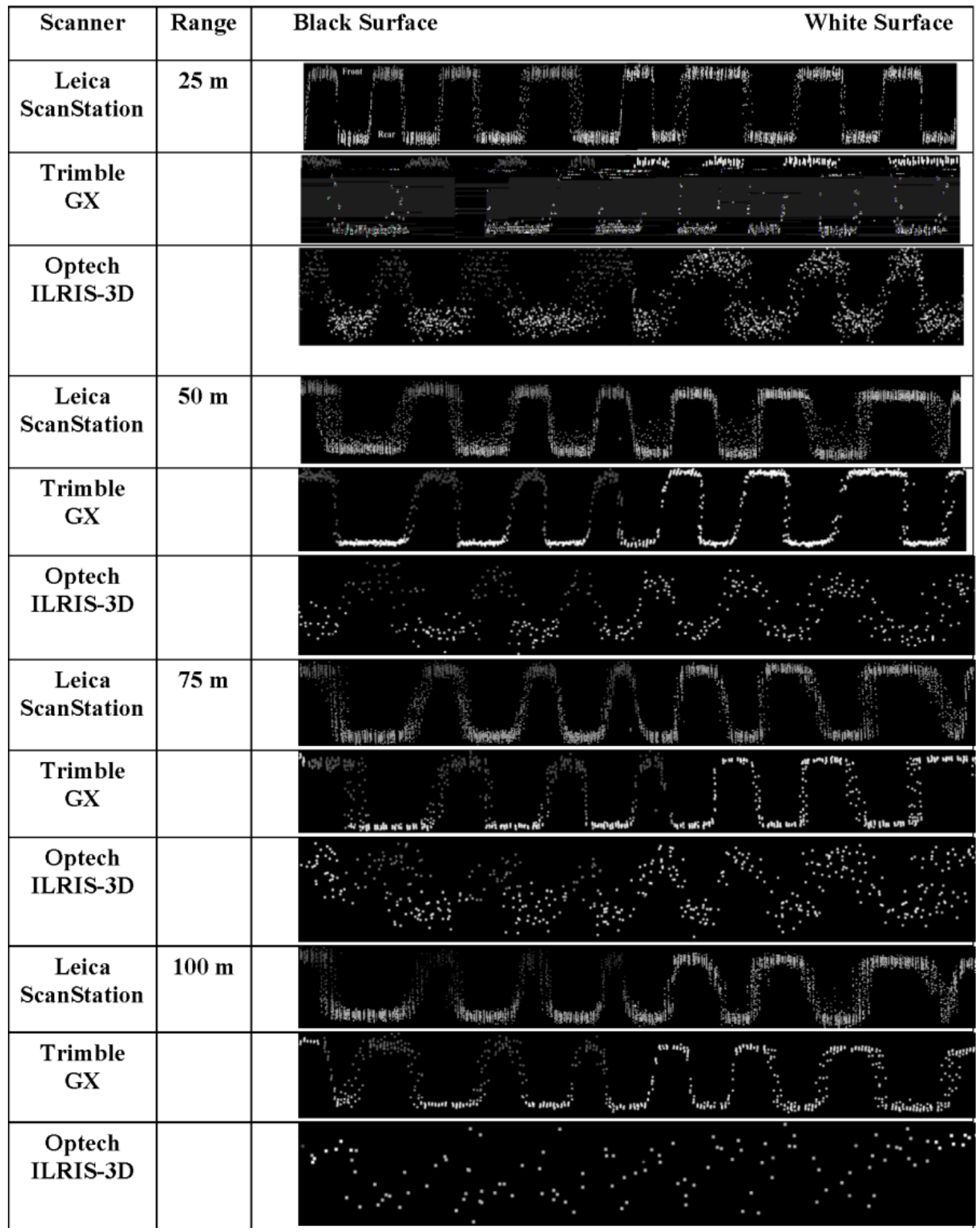




\subsection{Current LiDAR Applications in Civil Engineering}

Terrestrial LiDAR has been research and evaluated for the use in numerous civil engineering applications. Studies have shown that terrestrial LiDAR can provide useful metrics for bridge clearance issues, static deflection measurements and surface damage detection (Wanqiu and Shen-En 2011). The ability to accurately assess civil engineering application with limit public disturbance would greatly enhance the industry allowing for a cache of useful metrics.

Research pertaining to the reliability of crack detection with the incorporation of terrestrial LIDAR was conducted in 2008. The results showed that the particular terrestrial LiDAR unit (Trimble GS200 3D Scanner) allowed for accelerated inspection of suspect building surfaces, but trend to overestimate the width of the crack by more than 0.276 in $(7.0 \mathrm{~mm}$ ) (Laefer et al. 2010). The use of the terrestrial LiDAR system allowed for the creation of a permanent, which allowed for the results to be further evaluated at a later date or compare to future inspection results (Laefer et al. 2010).

When considering the costs required for the mandatory two year inspection and the limited amount of funding allocated towards infrastructure maintenance, the desired for a cost effective alternative to sensors instrumentation is necessary. For bridge clearance and static deflection analyses, terrestrial LiDAR has been shown to possess the capabilities to accurately assess the current condition of questionable bridges. Studies have shown that certain terrestrial LiDAR units are capable of accurately measuring both bridge clearance and static deflection to 0.125 in $(3.00 \mathrm{~mm}$ ) of the actual height (Wanqiu and Shen-En 2011). With the obtainable resolution of terrestrial LiDAR systems ever increasing, the ability to accurately detect minuet surface deviations is becoming possible, allowing for new applications of the remote sensing technology.

\subsection{Summary of Literature Review Sections}

From years of hard use and shrinking maintenance funding, the United States' bridge infrastructure system has been rapidly deteriorating. Of the nation's approximately 600,000 bridges, nearly 87,000 bridges possess structurally deficient characteristics. 
Recent events such as the Minneapolis I-35W and the San Francisco-Oakland Bay bridge collapses; the deteriorated state of the United States' bridge infrastructure system has only been highlighted.

When considering those nearly 87,000 structurally deficient bridges, a vast majority are constructed of reinforced concrete. Reinforced concrete material is the most widely used construction material worldwide, mainly due to the material's low initial cost and on-site construction capabilities. With such a widely used construction material, knowledge of deterioration mechanisms and related distresses are crucial. Common concrete deterioration mechanisms are surface cracking, spalling, scaling, delaminations, voids and expansion joint issues. These common concrete deterioration mechanisms are broken down into two categories, based on the defects location through the depth of the concrete. All the discussed deterioration mechanisms draw on each other, resulting in a complex network of cause and effect, making determination of cause difficult without taking into account every facet.

Currently, concrete deterioration is determined by visual inspection, which relies heavily on an inspector's experience, introducing variability into the equation due to inherent human nature. The introduction of variability during the visual inspection is an area of growing concern. By introducing remote sensing technologies, such as Light detection and ranging (LiDAR) systems, the reliance on visual interpretation would be greatly diminished, increasing the confidence of the inspection report.

LiDAR is currently used by numerous federal and state agencies to create accurate point clouds renderings, allowing for bridge inventory measures, work site surveys and as-built construction models. With numerous Department of Transportations (DOTs) already utilizing LiDAR systems as a part of their day-to-day operations, the knowledge and equipment is already available and just requires repurposing for collection of bridge condition metrics. LiDAR possesses the capabilities to evaluate surface defect on reinforced concrete bridge decks and could possibly expand to other CoRe structural elements. 
LiDAR systems consist of a transmitter (radiation source), a receiver (detector) as well as a control and data acquisition system (Chu 2011). The process in which LiDAR evaluates an object starts with the transmitter emitting light that travels through the air until an obstructed by an object with known reflectivity. The light then reflects off the object, which is detected by the receiver allowing for the control and data acquisition system to evaluate the distance and intensity of the obstruction. This process is completed millions of times resulting in a dense point cloud allowing for a virtual rendering of the obstruction.

Limitations associated with LiDAR are that the technology is a light-of-sight measurement tool and that non-reflective surfaces are unable to be measured. Due to the complex shape of a bridge system (deck, superstructure and substructure), the light-ofsight issue was an area of initial concern. However, by establishing a local coordinate system of vendor specific targets, multiple scan locations are able to be fused together illuminating the once shadowed surfaces. The presence of water is another area of high concern, being susceptible to environmental conditions reduces the versatility of the technology. The issue with water is the refraction, which causes the LiDAR transmitted light to bend and enter the water body and never reflect. With no reflected light, no data points can be collected, so as a resulted it is necessary to remove any standing water from an object prior to data acquisition. Once the two major limitations are overcome the LiDAR technology has the capability to produce surface profiles of a reinforced concrete bridge deck with millimeter precision.

Numerous performance evaluation testing has been conducted to validate the metrics of LiDAR. One performance evaluation conducted by the Advanced Highway Maintenance and Construction Technology (AHMCT) Research Center at the University of California, Davis was highly informational. AHMCT developed multiple test fixtures to evaluate commercially available LiDAR scanners for range precision, target recognition precision, resolution and the effects of target reflectivity and laser incidence (Hiremagalur et al. 2007). The test concluded that LiDAR has the capability of producing data with 
millimeter precision for range and target recognition, but is highly dependent on the reflectivity of the material.

Past research has evaluated the reliability commercially available terrestrial LiDAR systems as it pertains to estimate of bridge clearances, static deflection measurements and surface damage detection. The results from the evaluated terrestrial LiDAR systems have shown that the ability to accurately assess a surface is highly dependent on the system's achievable resolution. Terrestrial LiDAR systems can accelerate the inspection practice of crack identification, but the remote sensing technology tends to overestimate the crack width by more than 0.276 in $(7.0 \mathrm{~mm})$ (Laefer et al. 2010). More recent findings have shown that terrestrial LiDAR systems have the ability to accurately assess bridge clearance and static deflections to a acceptable degree of confidence. The findings stated that the vertical measurements of the terrestrial LiDAR varied from the true measurements by only 0.125 in (3.00 mm) (Wanqiu and Shen-En 2011).

With further evaluation and testing, LiDAR could allow for federal and state inspectors to generate quantitative measures on surface defects present in reinforced concrete decks. The technology's millimeter precision would greatly enhance the inspection standards and can help decision makers efficiently allocate the shrinking maintenance funding. Currently, LiDAR has a high initial capital cost, but with numerous federal and state agencies already owning the equipment the potential for repurposing is there and could result in a rich surface defect detection metric. 


\section{Methodology}

\subsection{Equipment Used for testing}

There are currently three primary platforms for LiDAR; aerial, terrestrial and mobile.

For the performance evaluation document herein, a terrestrial LiDAR system was utilized for data acquisition. Due to the relatively high capital cost and limited budget, only two LiDAR systems were considered; a Riegl LMS-Z210ii and a Leica ScanStation C10. These two systems were available from Michigan Technological University's School of Technology and the Michigan Department of Transportation, respectively.

The Riegl LMS-Z210ii is a relatively outdated terrestrial LiDAR system and has been discontinued due to further technology advancements. The Leica ScanStation C10 is currently Leica's versatile terrestrial system and is in production. After reviewing each system's performance measures and additional literature regarding previous performance evaluations of similar systems, it was determined that both systems would be utilized separately for data acquisition. This decision was made due to the limited testing standards currently in existence for performance measures of LiDAR systems and the desire to demonstrate the advancements in the technology. By generating two individual point cloud data sets, the ability to produce respectable results were greatly enhanced and allowed for more quantifiable conclusions to be drawn from the findings.

\subsubsection{Riegl LMS-Z210ii}

The university-owned terrestrial LiDAR system, the Riegl LMS-Z210ii, is a rugged and fully portable sensor. This system was designed for rapid acquisition of high-density point clouds rendering high-quality three-dimensional images. A standard Windows notebook and a Riegl developed bundled software package, RiSCAN PRO, controls the systems and allows for real time viewing of the collected point cloud. With a field-ofview up to $80^{\circ}$ vertically and $360^{\circ}$ horizontally coupled with a measurement rate of up to 10,000 points per second, the unit would be able to generate fast high accuracy point clouds (Riegl 2011). Complementing the Riegl LMS-Z210ii's fast data acquisition was 
the true color optical camera, which was mounted to the top of the scanning unit. This optical camera allowed for the integration of true target surface color. The generated color channel allowed for straightforward texturing of three-dimensional models by unequivocal correspondence of color pixels and range measurements. Figure 3.1 shows the instrument setup.

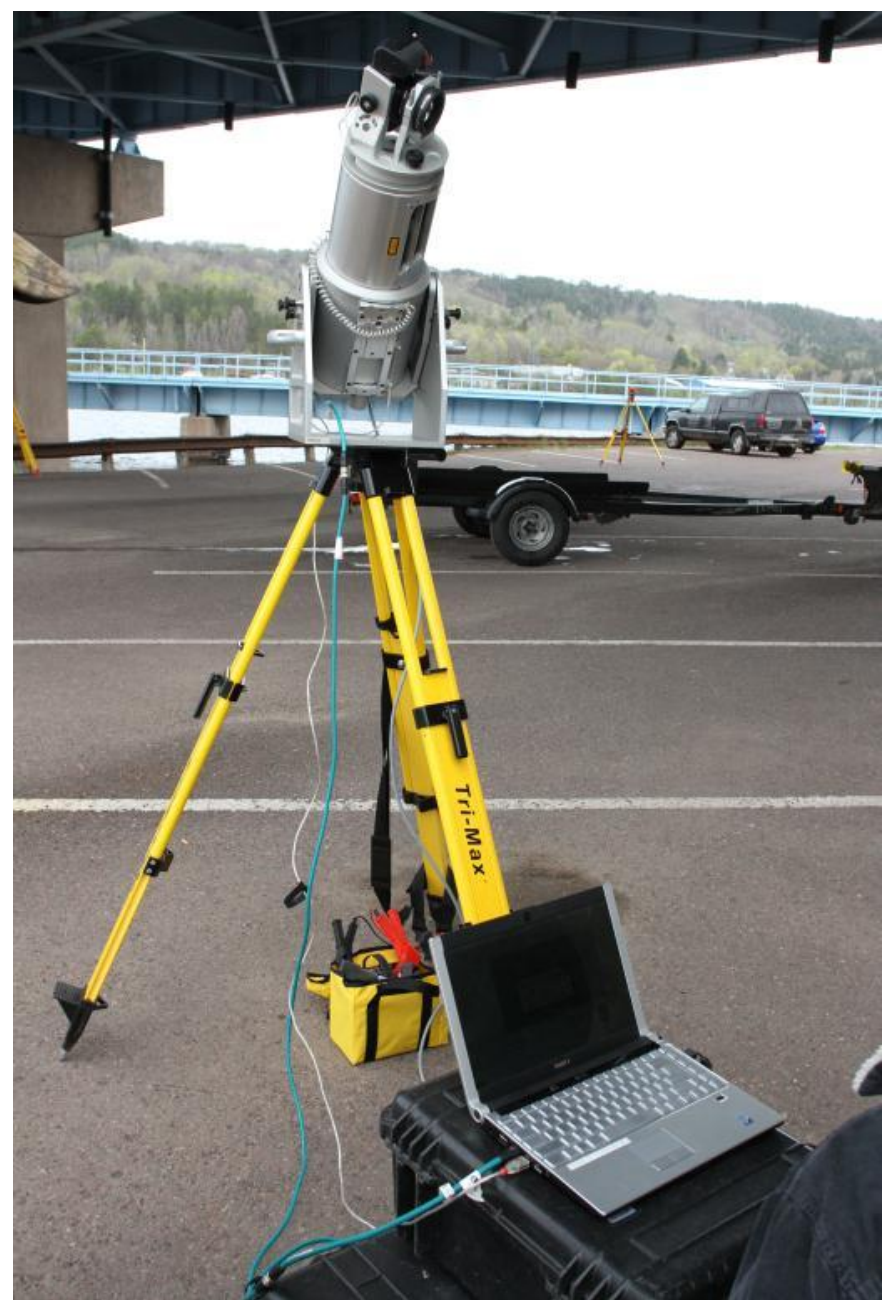

Figure 3.1: Riegl LMS-Z210ii system (Courtesy of Renee Oats) (See Appendix D)

The instrument is a pulsed, dual-axis compensated LiDAR unit in a monostatic configuration. For the LiDAR transmitter, a class 1, near infrared laser is utilized for eradiating the target object. Instrument specific performance specifications can be found in Table 3.1 (Riegl 2011). 
Table 3.1: Riegl LMS-Z210ii system performance (Riegl 2011)

\begin{tabular}{|l|l|}
\hline Maximum measurement range & $650 \mathrm{~m}$ \\
\hline Distance accuracy & $15 \mathrm{~mm}$ \\
\hline Distance precision & $10 \mathrm{~mm}$ \\
\hline Angular (horizontal/vertical) resolution & $87 \mu \mathrm{rad} / 87 \mu \mathrm{rad}$ \\
\hline Beam divergence & $2.7 \mathrm{mrad}$ \\
\hline
\end{tabular}

\subsubsection{Leica ScanStation C10}

Currently, MDOT utilizes the Leica ScanStation C10 as a surveying and inventory generator tool. By acquiring the services of experienced MDOT surveyors, Kelvin Wixtrom and Shawn Roy, the operational confidence produced negligible error in the data collection. This terrestrial LiDAR system, the Leica ScanStation C10 is an "All-inOne” High-Definition Surveying ${ }^{\mathrm{TM}}$ (HDS ${ }^{\mathrm{TM}}$ ) portable surveying instrument. With a full field-of-view, high-speed, high-accuracy, long range scanner with unmatched versatility allowed for rich point cloud generation of the all sampled bridges (Geosystems 2011). The instrument is a compact, pulsed, dual-axis compensated LiDAR unit with the following system details listed in Table 3.2 (Geosystems 2011). Figure 3.2 shows the instrument setup.

Table 3.2: Leica ScanStation C10 system performance (Geosystems 2011)

\begin{tabular}{|l|l|}
\hline Position accuracy & $6 \mathrm{~mm}$ \\
\hline Distance accuracy & $4 \mathrm{~mm}$ \\
\hline Angular (horizontal/vertical) resolution & $60 \mu \mathrm{rad} / 60 \mu \mathrm{rad}$ \\
\hline Modeled surface precision/noise & $2 \mathrm{~mm}$ \\
\hline Target acquisition & $2 \mathrm{~mm}$ std. deviation \\
\hline
\end{tabular}




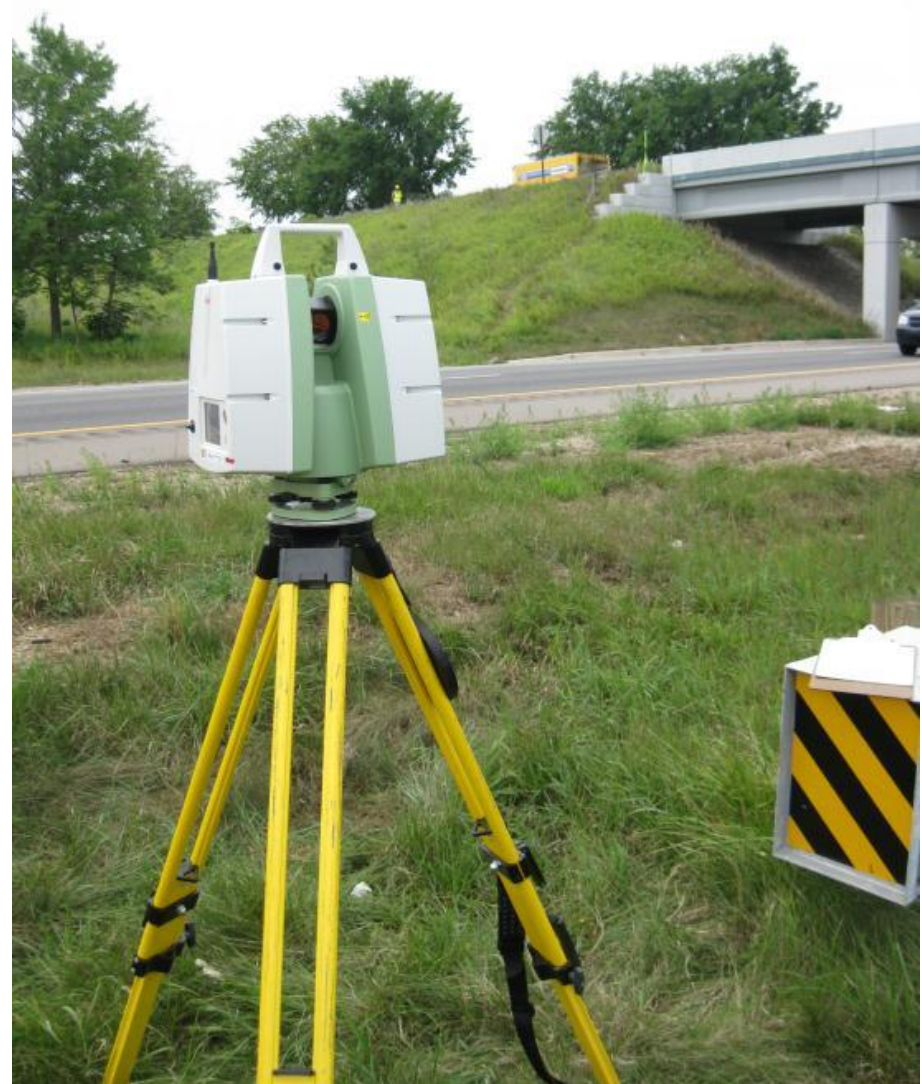

Figure 3.2: Leica ScanStation C10 system (Courtesy of MTRI) (See Appendix D)

All of the accomplished system performance measures listed above were achieved through the use of state-of-the-art components and proprietary software. The system utilizes a ns pulsed class 3R green laser transmitter for eradiating the target object. A vertically rotating mirror on a horizontally rotating base comprises the scanning optics projecting the transmitted laser over the selected field-of-view. The projected laser produces a minimal spot size of $4.5 \mathrm{~mm}$ or $7 \mathrm{~mm}$ over a distance of $50 \mathrm{~m}$ based on FWHH- and Gaussian-based testing, respectively. With the two systems defined, field demonstration locations were established.

\subsection{Full-scale Field Demonstration Selection}

The aim of the full-scale field demonstration selection process was to identify bridges that had varying degrees of degradation with the potential to be identified and quantified 
using multiple remote sensing technologies including Light Detection and Ranging (LiDAR). The evaluation of surface defect detection using LiDAR for bridge structural health monitoring was a portion of a larger venture incorporating multiple remote sensing technologies allowing for data fusion for an accurate generation of a structural health index. To accommodate the multiple remote sensing technologies, selection parameters were established requiring numerous forms of degradation to be present in the selected bridges. The end goal of the site selection was to identify three bridges within the state of Michigan that can be inspected (visual and detailed), tested, and evaluated using both traditional structural health monitoring techniques (strain gages, deflectometers, accelerometers, live load vehicles, hammer-sounding, chain-drag) for correlation as well as remote sensing technologies (thermal infrared, 3D optical bridge-evaluation system, radar, LiDAR)

To allow for a more comprehensive assessment of each technology, the preliminary selection parameter was defined accordingly by using the National Bridge Inventory (NBI) rating scale along with current Michigan Department of Transportation (MDOT) assessment practices. For completeness the NBI rating scale has been provided in Table 3.3. The three bridges were broken down into separate categories, "poor”, “fair”, and "satisfactory" each of which had correlating NBI deck ratings of four, five and six (or better), respectively (MDOT 2011b). Due to the nature of the tested remote sensing technologies a homogenous deck, superstructure and substructure material was preferred. To accomplish this additional parameter the candidate bridges for each category were separated by item "43: Main span(s) material type” of the MDOT structure inventory and appraisal form (such as pre-stressed concrete box beam versus steel continuous). Once the bridges were broken down into their main material types, it was determined the prestressed concrete I-girder material type bridges were to be further investigated due to the abundance of candidates in the three categories. Preliminary site visits and appraisals were conducted allowing for visual observation and validation of document deficiencies recorded in past inspection reports. 


\section{Table 3.3: NBI rating scale (MDOT 2011b) (See Appendix D)}

National Bridge Inventory Rating Scale:

9-Excellent Condition

8-Very Good Condition: No problems noted

7-Good Condition: Some minor problems

6- Satisfactory Condition: Structural elements show minor deterioration

5-Fair Condition: All primary structural elements are sound, but may have minor corrosion, cracking, or chipping. May include minor erosion on bridge piers.

4-Poor Condition: Advanced corrosion, deterioration, cracking, or chipping. Also, significant erosion of concrete bridge piers

3-Serious Condition: Corrosion, deterioration, cracking and chipping, or erosion of concrete bridge piers have seriously affected deck, superstructure, or substructure. Local failures possible.

2-Critical Condition: Advanced deterioration of deck, superstructure, or substructure. May have cracks in steel or concrete, or erosion may have removed substructure support. It may be necessary to close bridge until corrective action is taken.

1- "Imminent" Failure Condition: major deterioration or corrosion in deck, superstructure, or substructure, or obvious vertical or horizontal movement affecting structure stability. Bridge is closed to traffic, but corrective action may put it back in light service.

0-Failed Condition: Out of service, beyond corrective action.

Following the completion of the preliminary site visits, photographs were collects and organized. An image database was developed and then used to generate discussion about each remaining bridge and the suite of technologies' implementation capability. All the technologies were judged individually for each bridge with a focus on four criteria; presence of sensing deficiencies, accessibility, setup and sampling. To generate a complete evaluation of the four established criteria the deck top surface, bottom surface and bridge superstructure were individually assessed. Upon completion of the bridge selection discussion, three field demonstration locations along with a supplemental selection had been established each fulfilling the selection parameters for the three separate categories. The bridges selected were as follows:

- Mannsiding Road over US-127 north bound for the "Poor" selection

- Willow Road over US-23 for the "Fair" selection 
- Freer Road for the "Satisfactory" selection

- Mannsiding Road over US-127 south bound for the "Supplemental" selection

The field demonstration locations for the remote sensing technologies can be seen in Figure 3.3. The "Poor" and "Supplemental", "Fair", and "Satisfactory" locations are displayed in the provided figures as pin A, B, C, respectively. Due to the close proximity of the "Poor" and "Supplemental" bridge selections only one pin is displayed. Additional information for each selected field demonstration location is provided in the pertaining subsection and Appendix A.

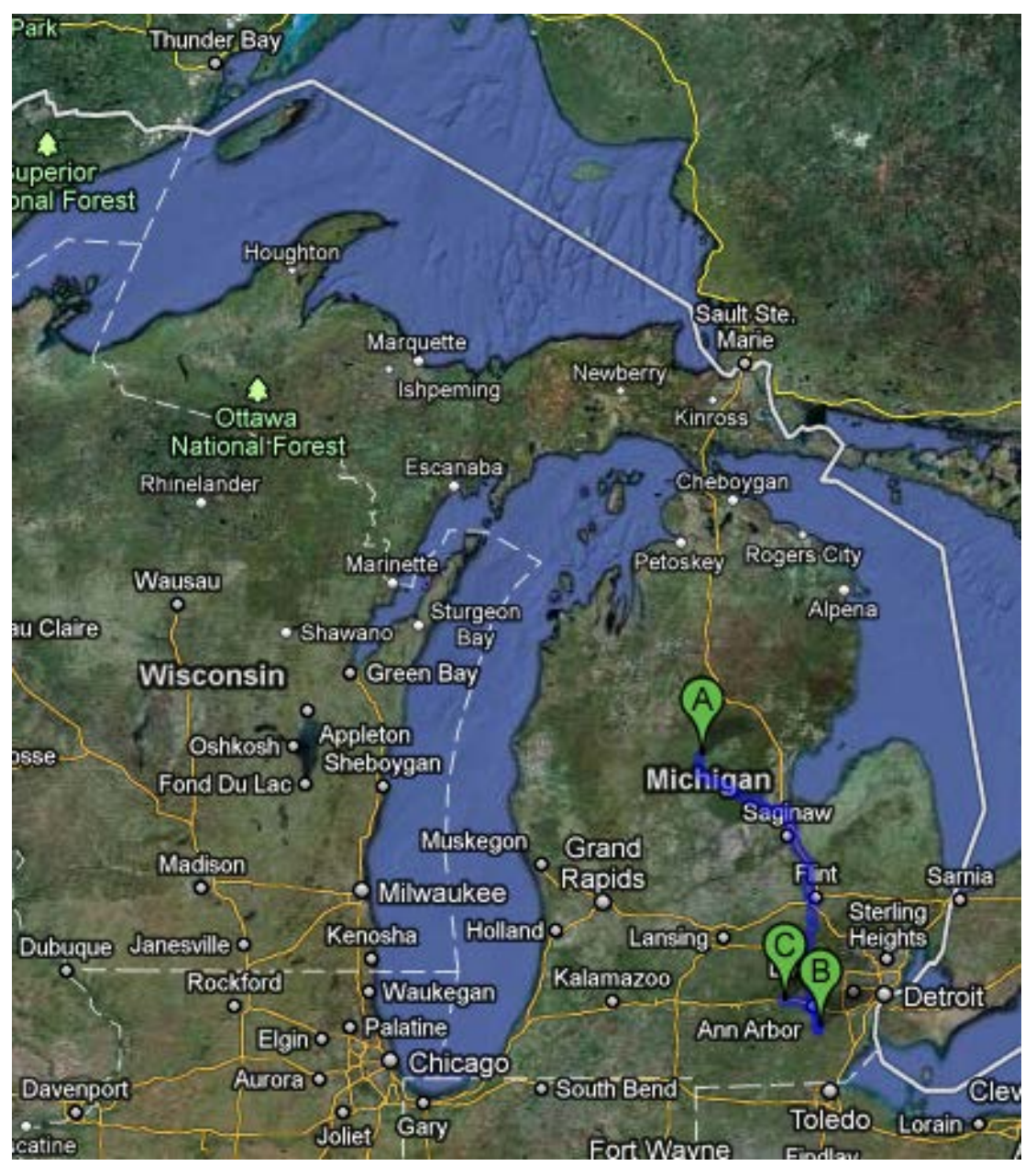

Figure 3.3: Field demonstration locations 


\subsection{1 “Poor” Bridge Selection}

The "Poor" bridge selection, MDOT structure nํㅜ 1713 - Mannsiding Road over US-127 north bound is located in Clare County approximately ten miles north of Clare, Michigan (Figure 3.4) and was determined to be the best candidate for field demonstration purposes.

The field demonstration candidate structure serves Mannsiding Road; a "Major Collector" road. The bridge was constructed in 1966 and is a three-span pre-stressed concrete multiple I-beam composite structure. The structure is $130 \mathrm{ft}-11$ in in length, 31 $\mathrm{ft}-2$ in in width, which translates into 26 in of riding surface. During 1996, the average daily traffic (ADT) over the structure was found to be 1,000 with 3\% being commercial (MDOT 2011b).

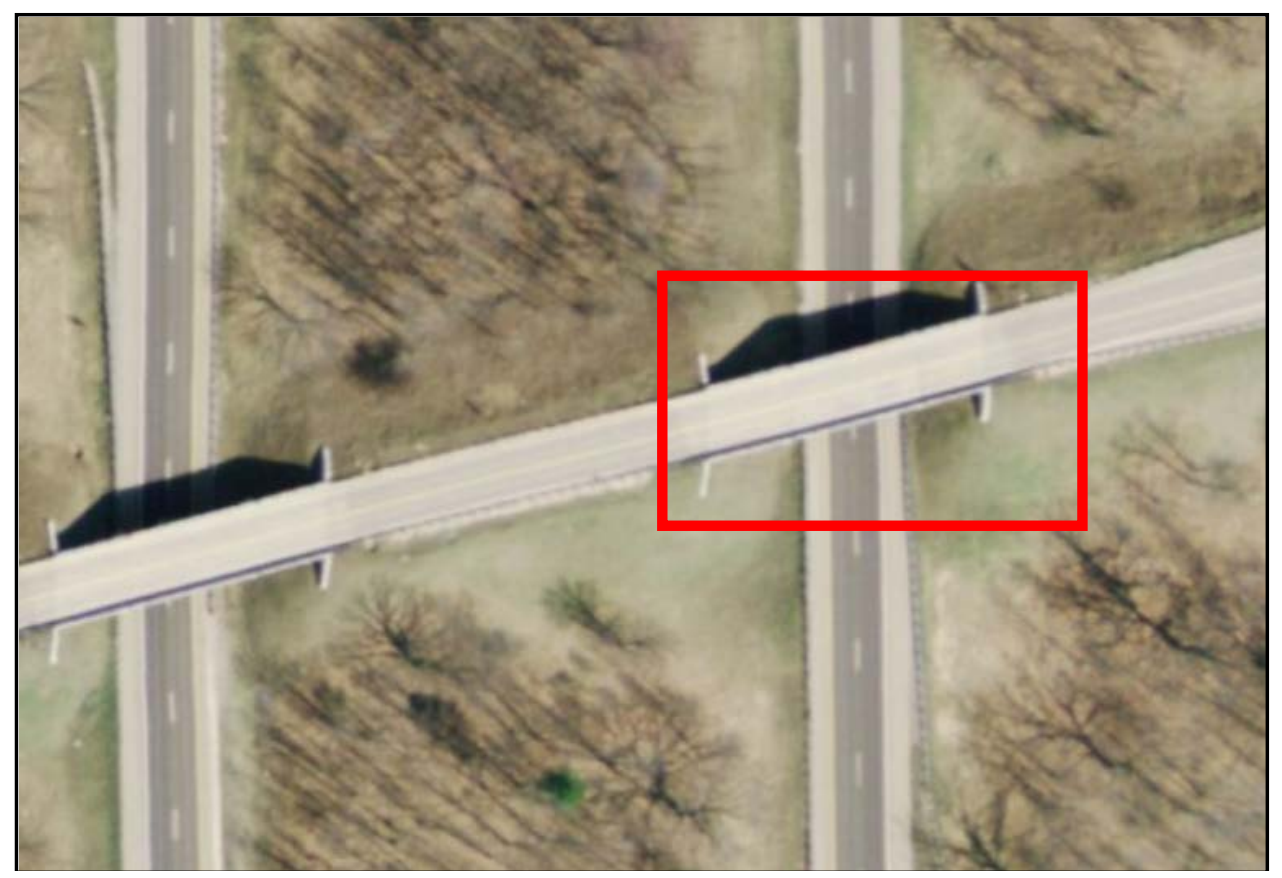

Figure 3.4: "Poor" bridge selection

Currently the bridge has no posted speed limit restriction. The crossing spans north bound US-127; a National Highway System (NHS) route that is not within any federalaid urban boundary. The bridge does not meet the desired minimum vertical clearance for NHS routes. The field demonstration location was located 2.3 miles south of M-61 or 
approximately 5.5 miles south of Harrison on US-127. The structure is part of an interchange serving the greater Harrison area, which is considered to be a rural environment. Figure 3.5 shows two photos of the bridge, one taken in 2008 during a MDOT scoping/inspection that provided detailed condition information, and the other by the research team during a site visit in June 2011. The structure is located in Hatton Township within Clare County.

The condition of the concrete deck surfaces, both top and bottom, were an area of major concern. A 2008 MDOT scoping inspection classified the deck with a NBI rating of "4". The scoping revealed that on the top surface of the concrete deck $176 \mathrm{ft}^{2}$ or $4.4 \%$ of the deck was delaminated. Additional testing on the bottom surface revealed that $623 \mathrm{ft}^{2}$ or $15 \%$ of the deck was in distress (MDOT 2011b). The deck also possessed light scaling throughout and numerous transverse, longitudinal and diagonal cracks were present. Additionally, several high-load hits have resulted in scrapes and spalls of the superstructure underside, but currently there is no sign of exposed reinforcing steel or pre-stressing strands. The bridge is scheduled for complete replacement in 2012-13. Additionally, during the on-site inspection of the selected "Poor" bridge, it was found to have a complementing twin bridge, Mannsiding Road south bound overpass. The collection of LiDAR data was completed allowing for an additional case study, but was not in the original scope of work. The Mannsiding Road south bound overpass bridge is described in further detail in the "Supplemental" bridge selection section. 

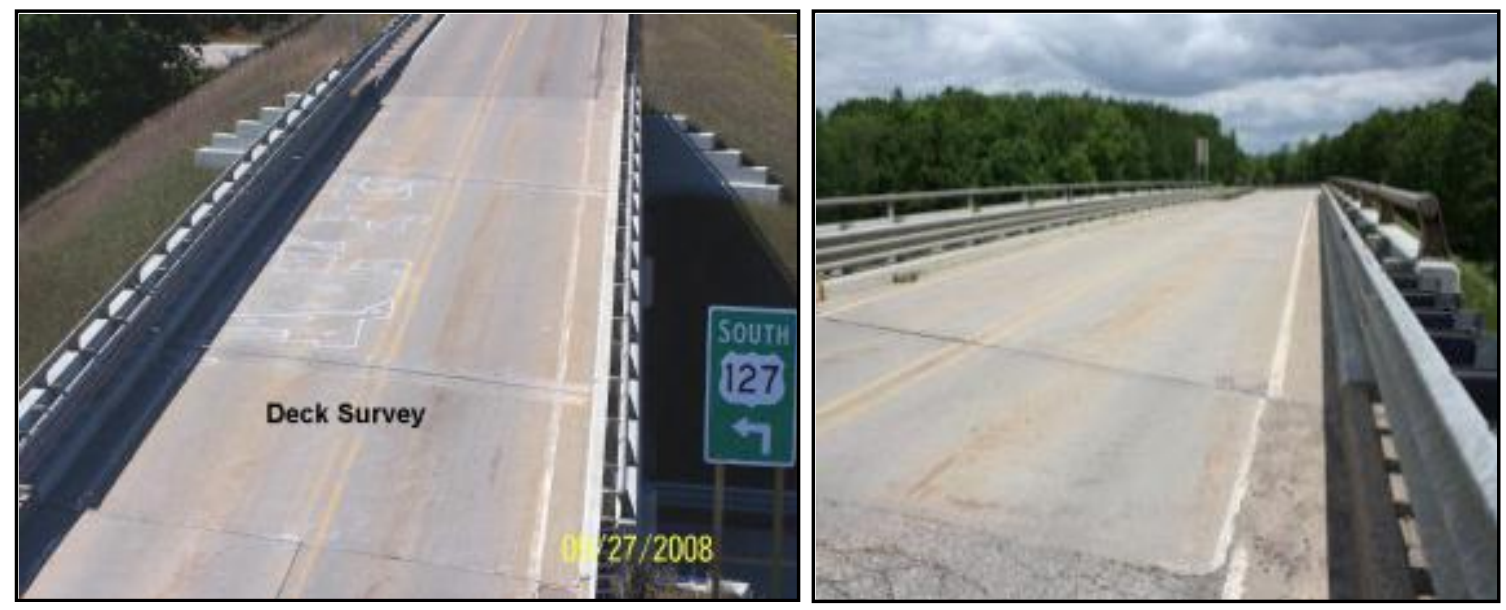

Figure 3.5: Mannsiding Road photographs (Courtesy of MTRI) (See Appendix D)

\subsection{2 “Fair" Bridge Selection}

MDOT structure nํㅜ 10892 - Willow Road over US-23 was selected for the "Fair" field demonstration bridge. The bridge is located in Washtenaw Country approximately three miles north of Milan, Michigan. An aerial photograph of the selected site is shown in Figure 3.6.

The "Fair” field demonstration structure serves Willow Road; a “Major Collector” road. The bridge was constructed in 1962 and is a four-span pre-stressed concrete multiple Ibeam composite structure. The structure is $209 \mathrm{ft}$ in length, $30 \mathrm{ft}-10$ in in width, which translates into $26 \mathrm{ft}$ of drivable surface with no availability for shoulder room. During 1997, the ADT over the structure was found to be 2,220 with 3\% being commercial (MDOT 2011b). Currently the bridge has no posted speed limit restriction. The crossing spans both north and south bound US-23; a NHS route that is not within any federal-aid urban boundary. The bridge does not meet the desired minimum vertical clearance for NHS routes. The structure is located in York Township within Washtenaw County. 


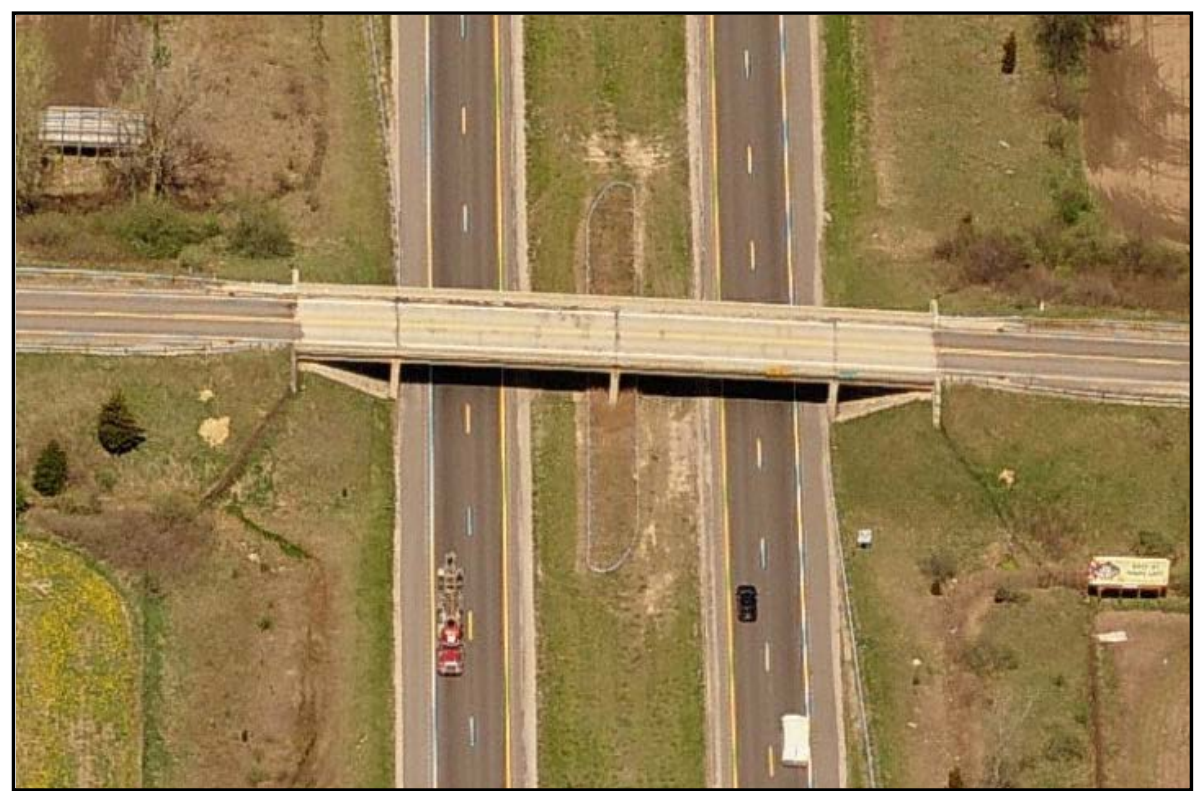

Figure 3.6: "Fair" bridge selection

The current condition of the deck surface is rated at a " 5 ” on the NBI scale. In 2010, the inspection report indicated that open transverse cracks, diagonal cracks and areas of delamination were present throughout the deck. Concrete patching had been completed to help minimize deterioration and prolong the service life of the bridge. Additionally, areas on the bridge superstructure displayed desired sensing deficiencies over both the north and south bound lanes. This is attributed to several high-load hits, which had resulted in scrapes and spalls, but there was no sign of exposed reinforcing steel or prestressing strands. Figure 3.7 shows a photograph of the current condition of the concrete bridge deck. 


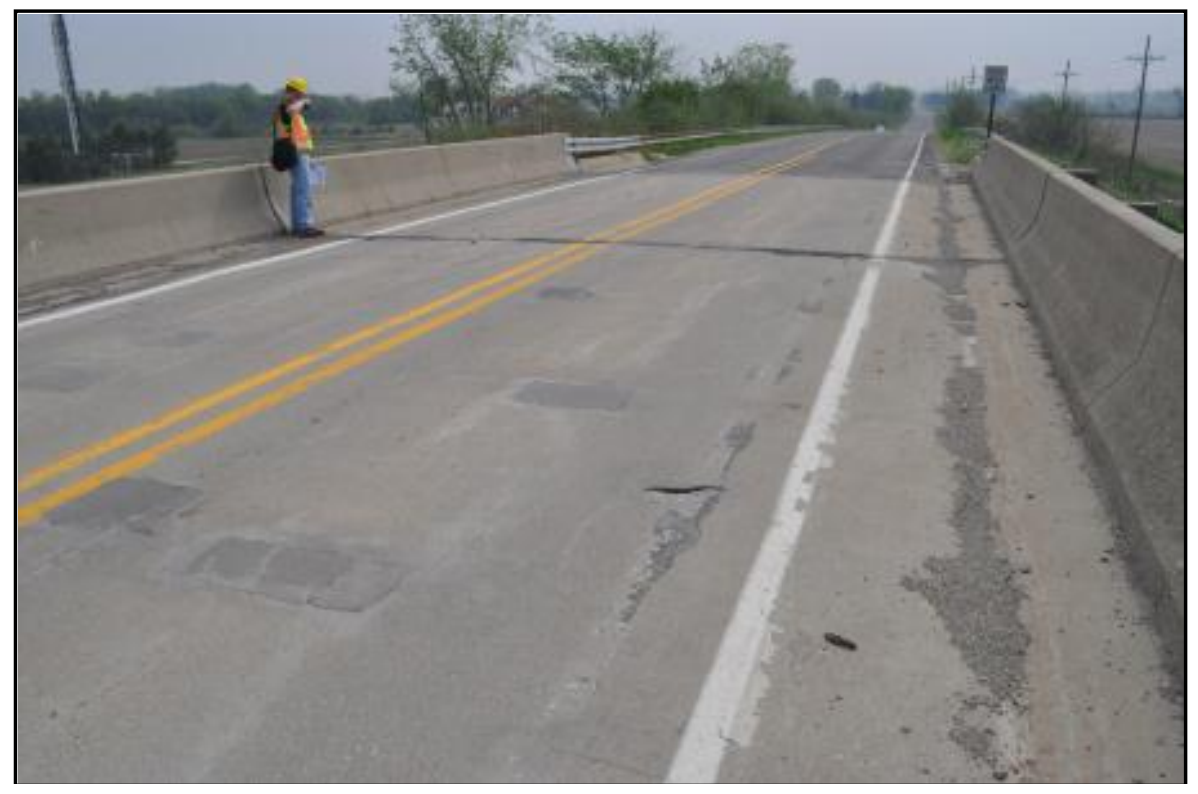

Figure 3.7: Willow Road photograph (Courtesy of MTRI) (See Appendix D)

\subsection{3 “Satisfactory" Bridge Selection}

Lastly, the "Satisfactory" bridge was chosen to be the MDOT structure n” 10940 - Freer Road over I-94 located in Washtenaw County, approximately one mile east of M-52 in Chelsea, Michigan. An aerial photograph of the selected site is shown in Figure 3.8.

The field demonstration candidate structure serves Freer Road; a "Major Collector" road. The bridge was constructed in 1960 and is a four-span pre-stressed concrete multiple Ibeam composite structure. The structure has dimensions of $209 \mathrm{ft}$ in length, $30 \mathrm{ft}-10 \mathrm{in}$ in width, which translates into $26 \mathrm{ft}$ of open roadway riding surface with no shoulder.

During 1997, the ADT over the structure was found to be 150 with 3\% being commercial (MDOT 2011b). 


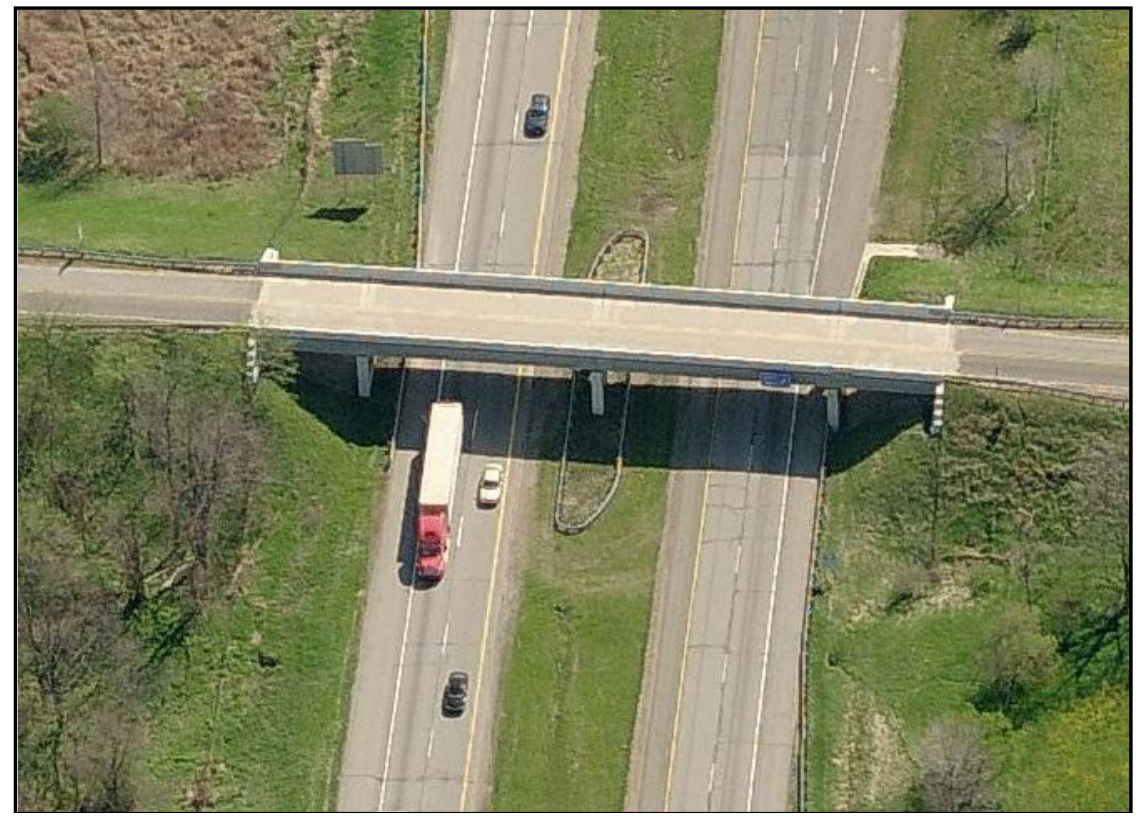

Figure 3.8: "Satisfactory" bridge selection

Due to the site's low ADT, this structure was the first to be tested, which allowed for the entire research team to work in an even safer environment while causing minimal disruption to local traffic going over the bridge. This site was the ideal location for the initial data collect to occur, allowing for the researchers to problem shoot any unforeseen issues prior to the remaining two bridges, which possessed higher ADT volumes.

Currently, the bridge has no posted speed limit restriction. The crossing spans both east and west bound I-94; a NHS route that is not within any federal-aid urban boundary. The bridge does meet the desired minimum vertical clearance for NHS routes with a measured clearance of $16 \mathrm{ft}$. The structure is located in Lima Township within Washtenaw County.

The NBI rating assigned to the concrete deck surface is a "6". In 2010, the inspection report indicated that there were several areas of concrete patching accompanied by few tight transverse and diagonal cracks present on the deck (MDOT 2011b). Concrete patches were applied to help minimize deterioration and prolong the service life of the bridge. The report indicated that there were also areas of interest on the superstructure where the concrete material had spalled and cracked. These areas of interest were located 
at the beam-end locations on the bottom flange. None of the spalled sections were deep enough revealing any reinforcing steel or pre-stressing strands allowing for corrosion to occur as shown in Figure 3.9.
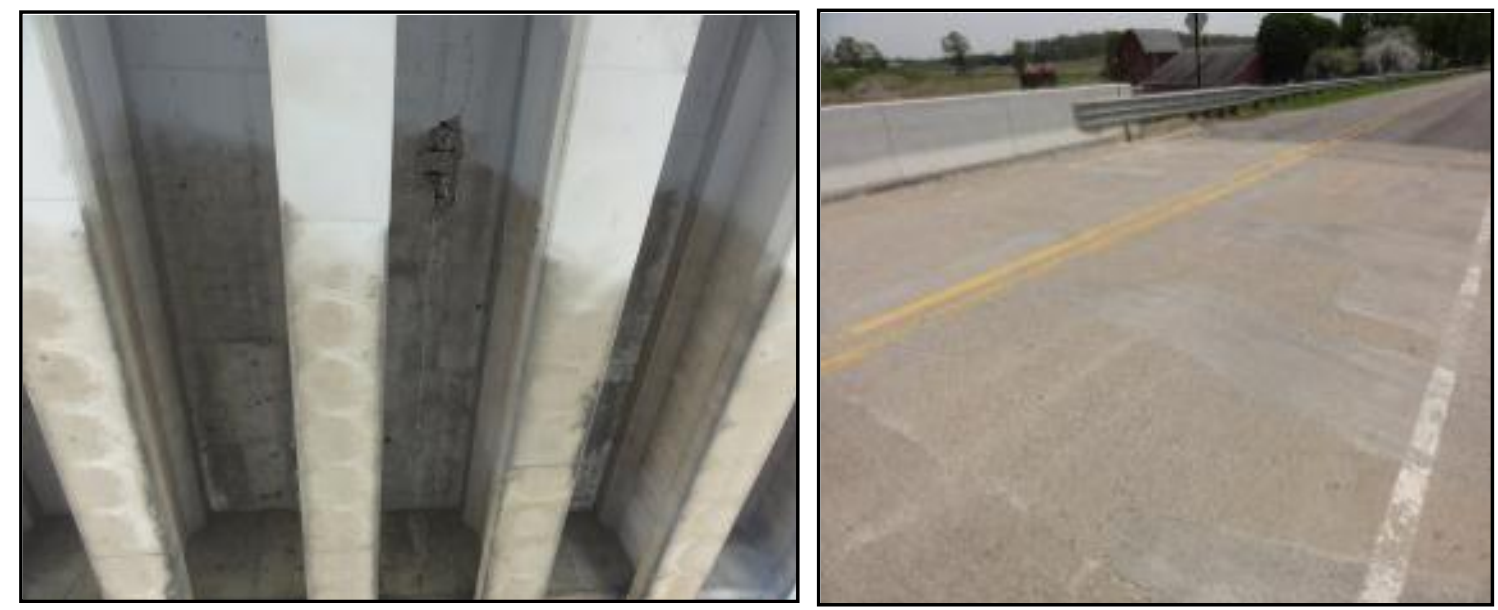

Figure 3.9: Freer Road photographs (Courtesy of MTRI) (See Appendix D)

\subsection{4 “Supplemental” Bridge Selection}

The "Supplemental" bridge selection, MDOT structure $\mathrm{n}^{0} 1712$ - Mannsiding Road over US-127 south bound is located in Clare County approximately ten miles north of Clare, Michigan and was not originally in the scope of work for the field demonstration.

However, the close proximity of the bridge to the "Poor" selection location allowed for data acquisition with no additional traffic disruption and permitting. An aerial photograph of the selected site is shown in Figure 3.10.

The field demonstration candidate structure serves Mannsiding Road; a "Major Collector" road. The bridge was constructed in 1966 and is a three-span pre-stressed concrete multiple I-beam composite structure. The structure is $130 \mathrm{ft}-11$ in in length, 31 $\mathrm{ft}-2$ in in width, which translates into $26 \mathrm{ft}$ of riding surface. During 1996, the ADT over the structure was found to be 1,000 with 3\% being commercial (MDOT 2011b). 


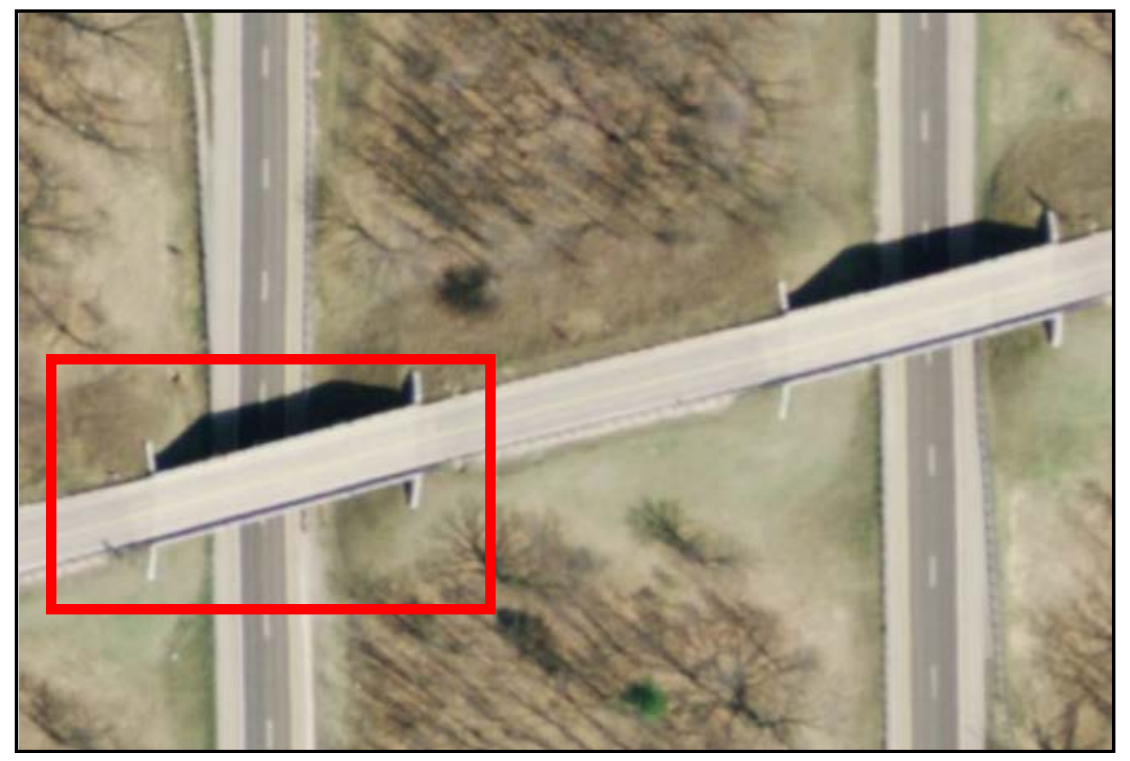

Figure 3.10: "Supplemental" bridge selection

Currently the bridge has no posted speed limit restriction. The crossing spans south bound US-127; a NHS route that is not within any federal-aid urban boundary. The bridge does not meet the desired minimum vertical clearance for NHS routes. The field demonstration location was located 2.3 miles south of M-61 or approximately 5.5 miles south of Harrison on US-127. The structure is part of an interchange serving the greater Harrison area, which is considered to be a rural environment. Figure 3.11 shows the general surface condition of the bridge deck. The structure is located in Hatton Township within Clare County.

When comparing the condition of the concrete deck surface, top and bottom, of the "Supplemental” and "Poor" bridge selections drastic differences in the state of deterioration were noted. The two bridges had been constructed at the same time, but large variations in the deteriorated state on the concrete deck are documented. An inspection conducted in 2010, classified the deck with a NBI rating of “7” (MDOT 2011b). The inspection notes stated that minor cracking and shallow scaling in the deck surface was present, however was not substantial enough to cause structural issues. When comparing the "Supplemental” bridge deck rating of "7" to the "Poor” bridge selection's deck rating of " 4 ” on the NBI scale, questions of construction procedure, inspection consistency and environmental effects were raised. 


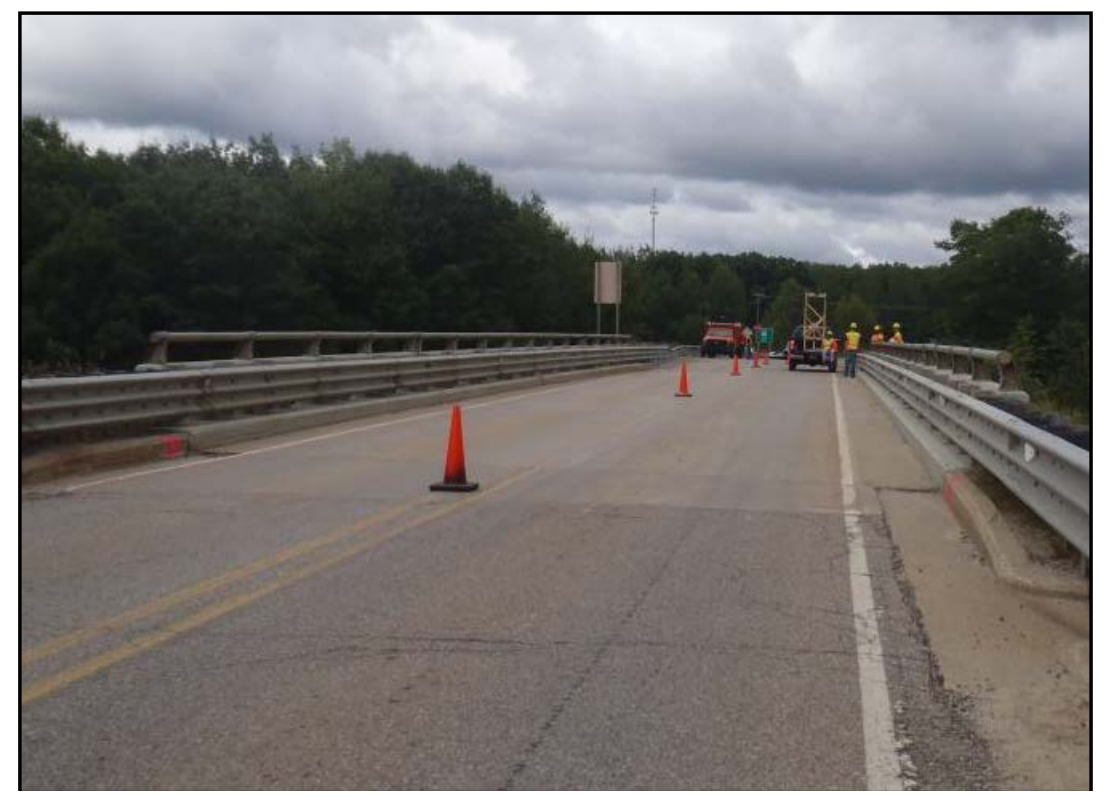

Figure 3.11: Mannsiding Road photographs (Courtesy of Renee Oats) (See Appendix D)

\subsection{Full-scale Field Demonstration Plan}

LiDAR can collect millions of data points in a single pass of a suspect object, allowing for detailed analysis to be completed when compared to traditional surveying techniques. Due to the fact that LiDAR uses reflected energy to generate a virtual surface of the suspect object, line-of-sight is required. To produce a complete three-dimensional rendering of a complex feature numerous collection locations of the device are required illuminating all the surfaces of the object. If repositioning of the device is not done the faces not captured within the line-of-sight will produce "shadows" or "blind spots" in the generated point cloud. These locations within the point cloud will be represented as empty space containing limited data points. Additionally, because light is the primary form of energy broadcasted over the selected field for object measurement, water is a concern. Fundamentally as light enters a body of water the wave bends as it enters a medium of another density. During this transition, the speed of the energy wave changes, changing the angle of the incident wave relative to the water surface. This phenomenon is called refraction, which causes the transmitted LiDAR laser beam to defuse and result in limited energy return to the LiDAR receiver. The result is a false "blind spot" within 
the point cloud. With this basic knowledge a general field development plan was developed for a generic bridge situation.

Prior to initializing the LiDAR data collection, a minimum of four points of commonality had to be established to triangulate and validate a local coordinate system. These points of commonality were represented as vendor specific targets mounted on sturdy tripods. Each LiDAR unit required different targets, because of varying manufacturer requirements. As a result, two local coordinate systems were established. The Leica ScanStation C10 required vendor specific targets, while the Riegl LMS-Z210ii used retro-reflective surveying prisms. The two different targets can be seen in Figure 3.12.
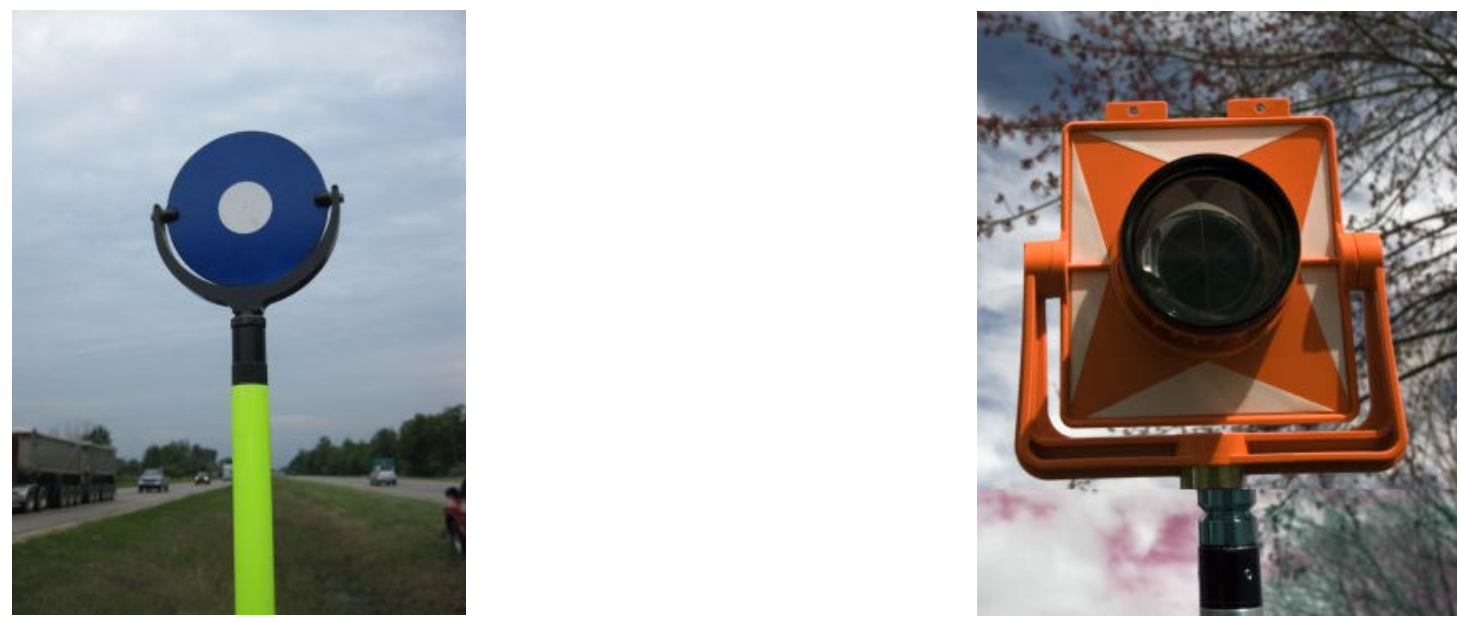

Figure 3.12: Vendor specific targets (Leica to left, Riegl to right) (Courtesy of MTRI) (See Appendix D)

After performing a visual inspection of the bridges, it was determined that due to the complex lines of the bridge superstructure multiple scan locations were required. However, to generate a local coordinate system that all the scan locations could be referenced to, additional points of commonality had to be established. On average eight points of commonality were established during the field demonstrations and by creating these points of commonality, the independent LiDAR point clouds were referenced in a common local coordinate system allowing for a layered three-dimensional rendering eliminating the presence of "shadows". If more than four points of commonality were 
visible from the position of the LiDAR system, higher point precision was obtainable with reference to the local coordinate system.

Once the local coordinate system was established, the bridge surface needed to be inspected for standing water ensuring limited generation of false "blind spots". Additionally, debris was removed from the concrete deck allowing for limited artifacts to be generated within the point cloud developing false features on the deck surface. With the standing water and debris removed from the bridge surface the data acquisition commenced.

Testing took approximately four hours, which included setup of six to 12 separate scan locations and demobilization. Scans were collected in units of international feet with both the Riegl LMS-Z210ii and the Leica ScanStation C10. A sample scan location map was generated allowing for limited on-site selection of the scan locations, which accelerated the data collection process. This sample scan location map can be seen in Figure 3.13. For the Leica ScanStation C10 detailed field sketches were provided to the research team upon completion of the data acquisition, which are available in Appendix B.

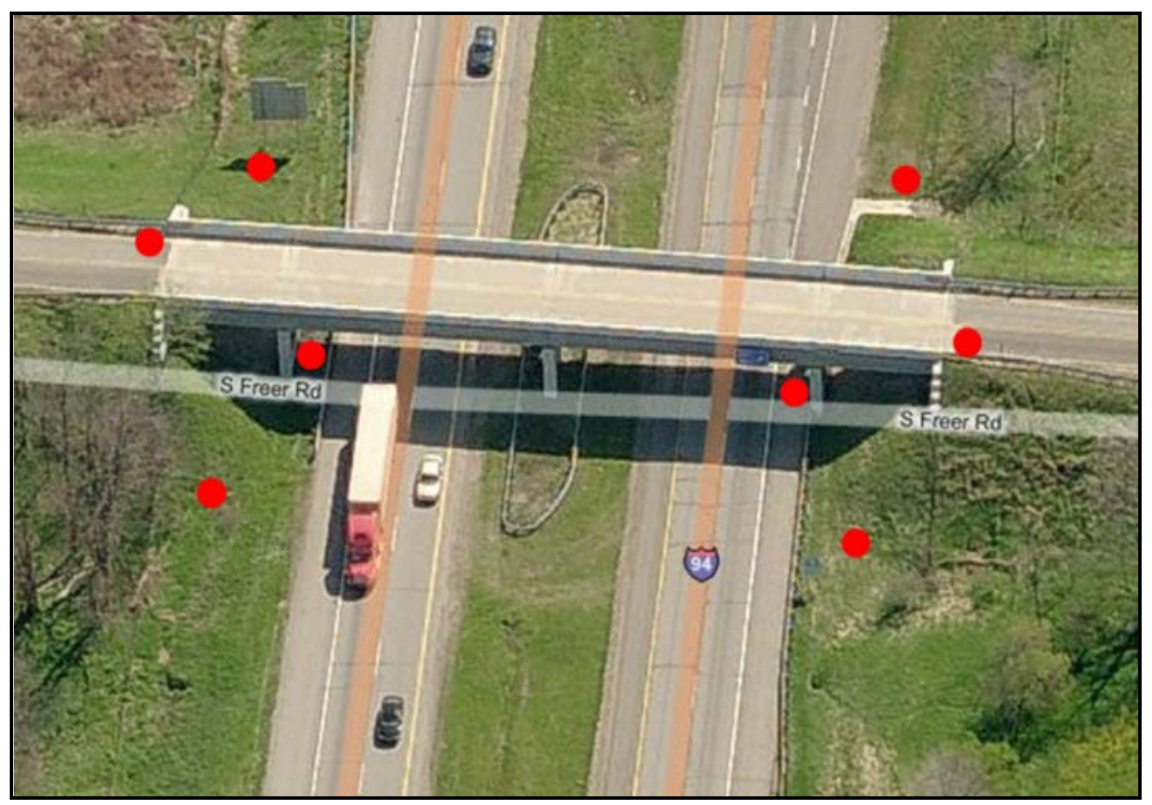

Figure 3.13: Sample LiDAR collection locations (red dots are LiDAR setups) 
When the equipment positions were fixed, an initial LiDAR scan of the entire surrounding area lasting approximately five minutes was completed. This overview scan allowed for the local coordinated system to be established by referencing the points of commonality within the image by identifying the center of the retro reflective prisms or vendor specific targets. Once the points were located, a more detail scan at a higher resolution lasting approximately ten minutes was completed by selecting a window of interest from the initial rendered image. The previously discussed process was then repeated for the remaining scan locations allowing for a final three-dimensional rendering of the subject bridge.

\subsection{Post-processing Software Selection}

For determination and evaluation of the surface defects present in the acquired point cloud data from the field demonstration locations, several modeling programs were considered. The modeling programs that were considered were Certainty 3D's TopoDOT modeling software, Quick Terrain Modeler and VR Cardinal Systems point cloud software. Selection criteria were established to help evaluate the commercially available software packages for LiDAR data processing. User-friendliness, cost, accuracy, what the modeling program was designed for and current federal and state agency uses were the five main criteria used to evaluate the multiple modeling programs.

The methods of analysis and algorithms utilized by a program greatly affect the obtainable precision and accuracy of the post-processed point could data. The issue arises when trying to evaluate a program's accuracy and precision, because of the proprietary coding utilized to execute the requested task available within the user interface. To select appropriate modeling software, the associated post-processing point cloud accuracy and precision of the program and proper procedures were requested for data extraction.

Certainty 3D’s TopoDOT modeling software is a Bentley MicroStation compatible software package, which utilizes Bentley developed algorithms to execute self-developed functions (Certainty 2011). Currently, TopoDOT is used by 40 separate transportation 
agencies and was initially developed to seamlessly post-process Riegl RiScan Pro projects. However, the increase in demand for a flexible post-processing software package that is compatible with numerous LiDAR systems, the system has incorporated a generic LAS LiDAR data file registration function. A LAS data file is a universally accepted LiDAR file form, which most LiDAR system can produce. The software is geared towards civil infrastructure point cloud data, both terrestrial and mobile LiDAR data can be imported into the user interface allowing for additional user refinement. The user-interface is identical to that of MicroStation and allows for easy acceptance into the civil engineering field due to the pre-existing knowledge base. TopoDOT allowed for all the established criteria to be satisfied except for the associated maintenance fee.

Quick Terrain Modeler is a LiDAR post-processing software, which is relatively inexpensive and user-friendly. Initially, the software was developed to evaluate and extract information from aerial LiDAR data sets for topography and land surveying (Imagery 2011). With the software originally designed for aerial LiDAR data, the ability to navigate around a complex three-dimensional surface was difficult. When considering the inexpensive, user-friendly interface of the software, the navigation issue was a minor detail.

When evaluating the VR Cardinal System's point cloud software, the design of the user interface was not intuitive. The user interface required extensive computer operation knowledge and program specific training to properly evaluate a point cloud data set. This software was dismissed from consideration due to the difficult user-interface. This evaluation of the modeling software is in no way implying that the software is poor, but was rather not appropriate for the desired action items of the research.

Upon conclusion of the software evaluation, Certainty 3D's TopoDOT modeling software was deemed the most effective at performing the desired post-processing techniques. However, the Quick Terrain Modeler was deemed necessary to acquire due to the lack of available funding to purchase a license for TopoDOT. Certainty 3D did provide an extended free trial of the TopoDOT software to perform the critical data 
extraction. The Quick Terrain Modeler was used later in the project timeline, when additional data separation and extraction was necessary to further evaluate the technologies potential.

\subsection{Certainty 3D's TopoDOT Modeling Software}

Prior to reception of the fused point clouds for each bridge, registration and georeferencing was completed on the Leica ScanStation C10 data, which was performed by MDOT. The data sets generated with the Leica ScanStation C10 were geo-referenced to the State Plane coordinate system, Michigan South zone 2113, High Accuracy Reference Network 1983 generated from MDOT’s high-accuracy Leica global positioning system (GPS), as shown in Figure 3.14. The vendor specific targets were the points georeferenced, because the targets were constant from scan to scan. By geo-referencing the data, the point clouds were projected and layered with real time satellite imagery and high-resolution optical images allowing for visual comparison. With the incorporation of optical imagery, determination of potential defects was established allowing for the user to disregard artifacts within the data, which could produce false positives. Additionally, MDOT assigned boundary conditions to the point clouds eliminating unnecessary data not associated with the bridge. 

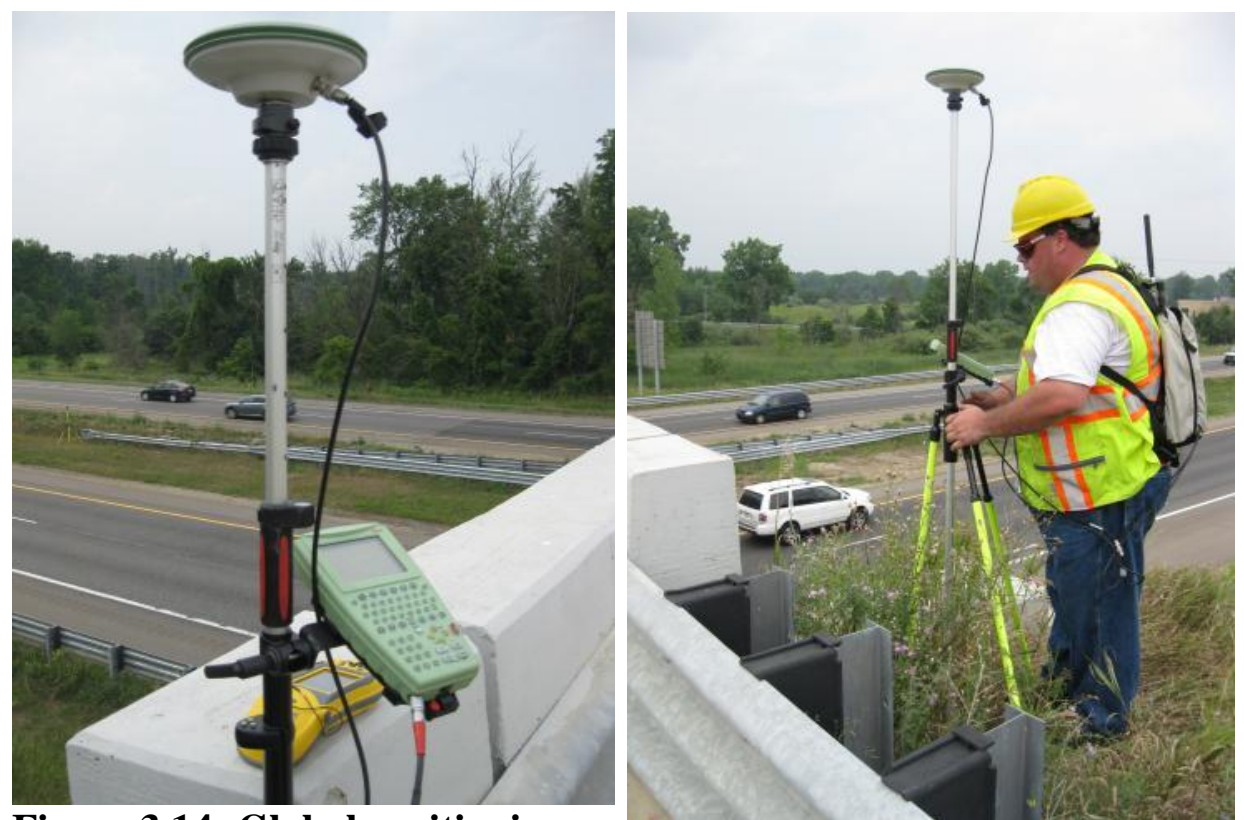

Figure 3.14: Global positioning system tor geo-rererencing (Courtesy of MTRI) (See Appendix D)

The Riegl LMS-Z210ii data unfortunately was corrupted beyond repair, due to unforeseen issues in the establishment of the local coordinate system. To clarify, the Riegl LMS-Z210ii point clouds produced from the individual scan locations were misaligned in all three axes, resulting in the inability to merge the data. However, data produced from the Leica ScanStation C10 was deemed sufficient for the goals of this research. The separate Leica ScanStation C10 data was provided in an LAS file format upon completion of the geo-referencing and boundaries were applied.

Before data filtering commenced, issues arose with available computing memory limiting the amount of displayable data points. With LiDAR having the capabilities of capturing millions of data points within a single scan, the size of the fused bridge data ranged from 2.0 gigabytes to 6.4 gigabytes. That large file size required an immense amount of available memory to display an individual bridge at full point cloud density. As a result, a computer with an Intel i5 processor with an additional 8.0 gigabytes of memory was purchased allowing for an increase in displayable point cloud density. It is prudent to point out that even with the hardware enhancement, the bridge point clouds in their 
entirety were still unable to be displayed. However, this limitation was software related, ensuring ample, residual memory to complete requested tasks efficiently.

With the hardware issues resolved and the point cloud data received, post-processing of the data commenced. As described in the previous section, Certainty 3D’s TopoDOT modeling software was selected to further post-process the field demonstration data (Certainty 2011). Originally designed for civil engineering applications, the ability to extract individual features from the raw point cloud data was effortless with TopoDOT. When first loading the TopoDOT program, the user was prompt to generate a unique file name and establish a file type. Figure 3.15 shows the user prompt necessary to start LiDAR processing. A critical step was to define the file type as a seed 3D file, which allows the user to edit the data within three-dimensional space. To define the file type a browse button located on the lower, right corner of the user prompt was utilized. With the desired file name established and proper file type, the file was saved.

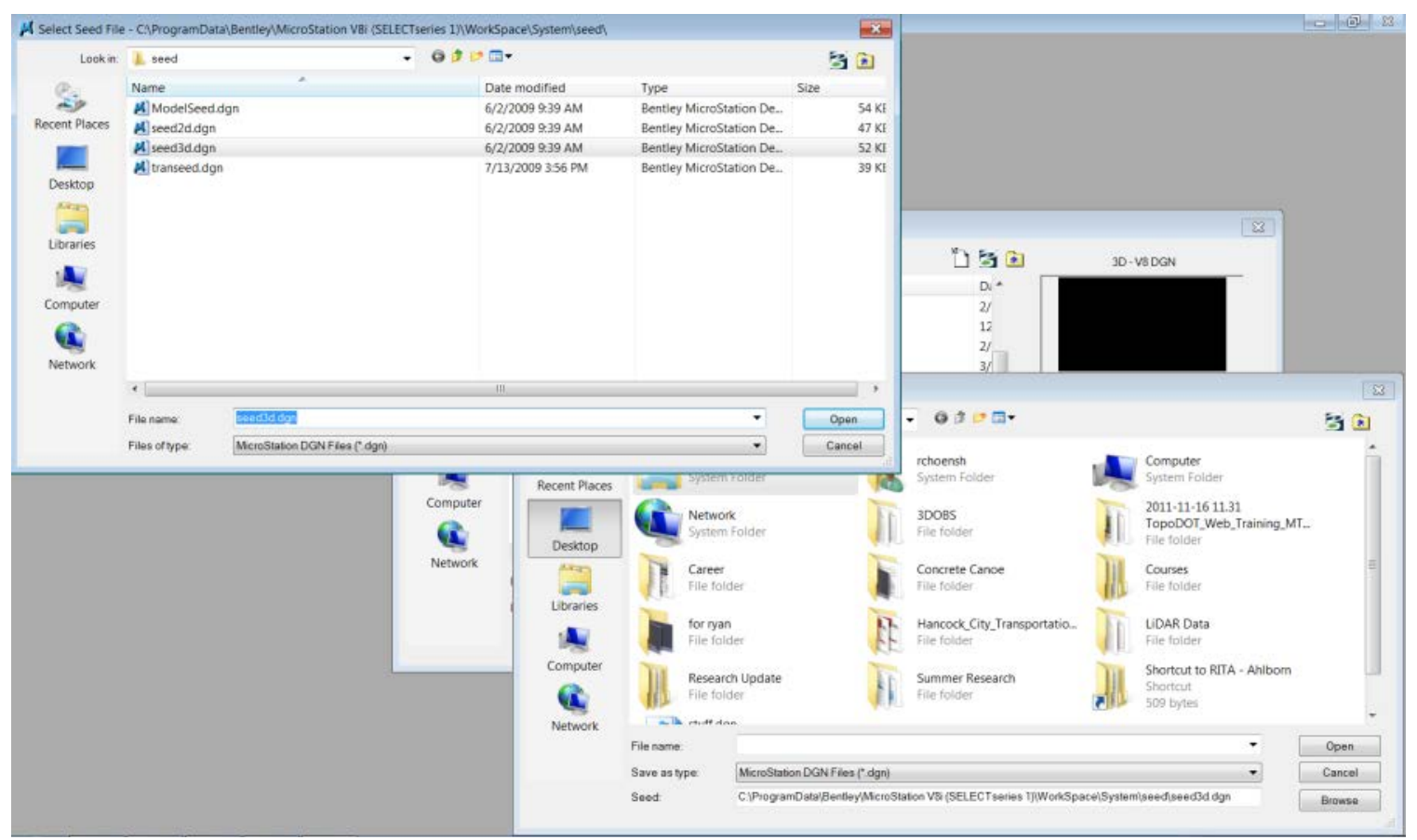

Figure 3.15: TopoDOT initializing user prompt (Certainty 2011) (See Appendix D) 
Now within the user interface, the user had to execute the TopoDOT software package. With TopoDOT being a Bentley MicroStation compatible software add-on, the program was required to be active within the user interface. Activation of TopoDOT was accomplished by finding the “TopoDOT $(N)$ ” drop down bar and selecting Start TopoDOT (Certainty 2011). A text window appeared and verified the user license was valid, as shown in Figure 3.16. Returning to the user interface, a toolbar on the left hand side appeared labeled “TopoDOT (N)”, as shown in Figure 3.17. The toolbar contained all Certainty 3D’s LiDAR developed post-processing functions.

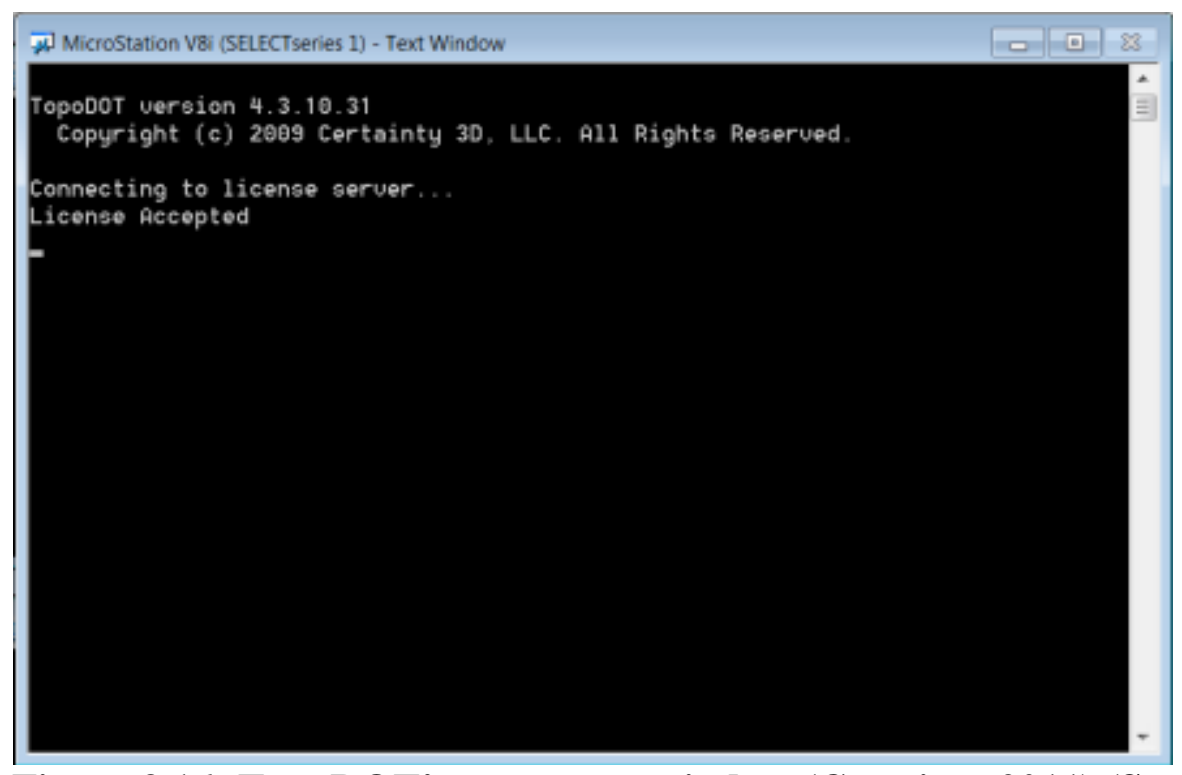

Figure 3.16: TopoDOT’s status text window (Certainty 2011) (See Appendix D)

Once TopoDOT was running, the point clouds were able to be imported individually and key extractions performed. To properly import the data, the desired LAS file was registered with TopoDOT by generating a Project.tvw file. This task was completed by using the "LAS Registration Command" icon found within the TopoDOT (N) toolbar. With the registration file created, file import parameters were established allowing for the LAS file to be scaled and geo-referenced. The import file parameters are established by locating the "File Settings" icon within the “TopoDOT (N)" drop down menu (Certainty 2011). The Leica data as described in an earlier section had units of international feet, which were required to be input in the "File Settings" user prompt, as shown in Figure 
3.18. Additionally, within the "File Settings" users prompt the ability to set the data display rate was available. The ability to down sample the full data set allowed the entire bridge to be displayed at a slightly less resolution. However, requested features were still able to be extracted at full resolution. As alluded to earlier, even with the hardware upgrade, the file size of an individual bridge exceeded the software's point cloud display limit, so down sampling was necessary.

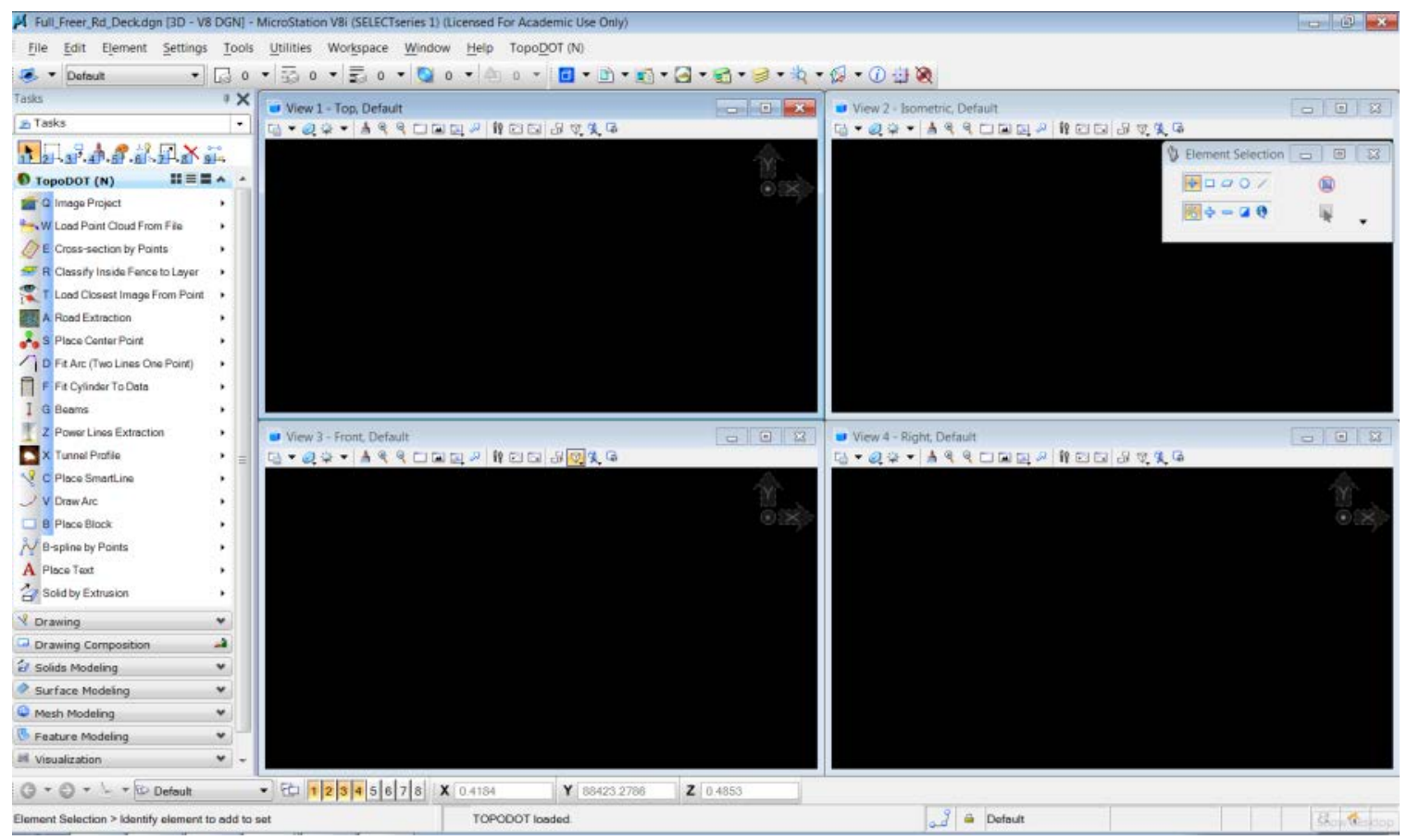

Figure 3.17: MicroStation user interface with TopoDOT activated (Certainty 2011) (See Appendix D) 


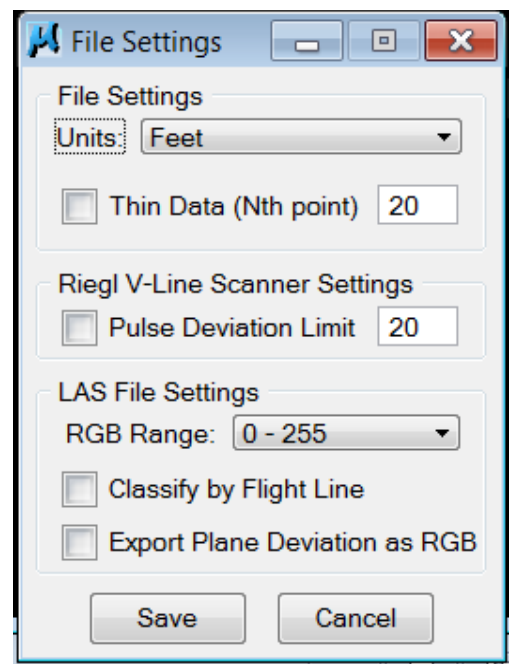

Figure 3.18: Import file settings for establishing import parameters (Certainty 2011) (See Appendix D)

With the import parameters applied, display parameters were required to be established. Found under the "Settings" drop down menu, the "Design File” icon was selected to display the user prompt to apply the desired display units, as shown in Figure 3.19. This function allowed for the user to select the desired units, the point cloud would be displayed at, by converting the import file accordingly. The import and design file parameters are critical steps, which if done incorrectly resulted in geo-reference scaling issues.

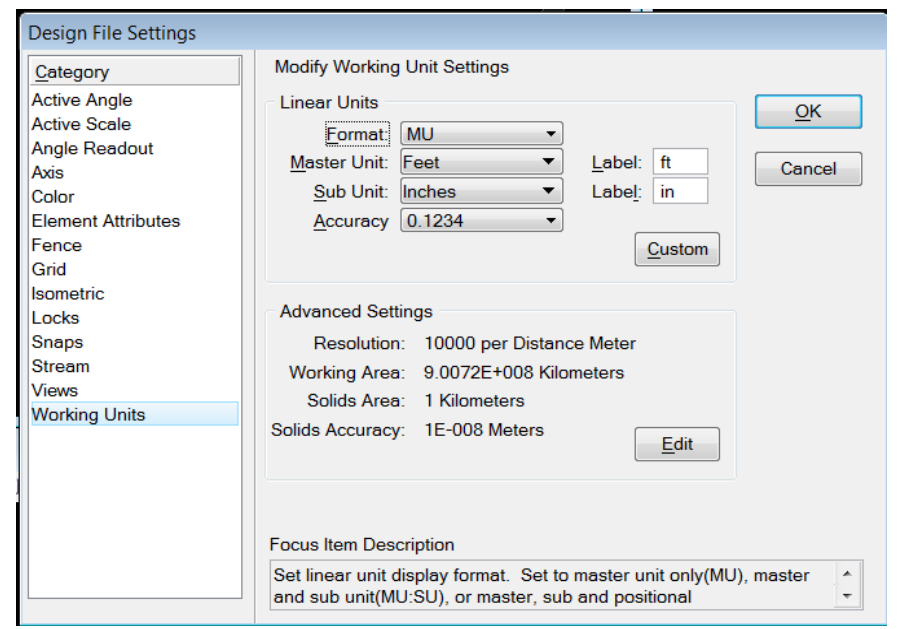

Figure 3.19: Design file settings for establishing display parameters (Certainty 2011) (See Appendix D) 
With all the import and design file parameters set the LAS files were imported by entering into the "TopoDOT (N)" toolbar, locating the "Load Point Cloud from File", executing the "Load Point Cloud from File" function and selecting the desired LAS file, as shown in Figure 3.20 (Certainty 2011). To ensure that the requested data had been fully displayed to the desired sampling rate, the user returned to the text window and confirmed that the number of total points visible did not end in multiple nines (9) s, as shown in Figure 3.21. When this was the case, the quantity of points requested exceeded the allowable software limits and further down sampling was necessary. The result of requesting additional points to be displayed than allowable was a program failure and required TopoDOT to be reopened. This was an iterative process.

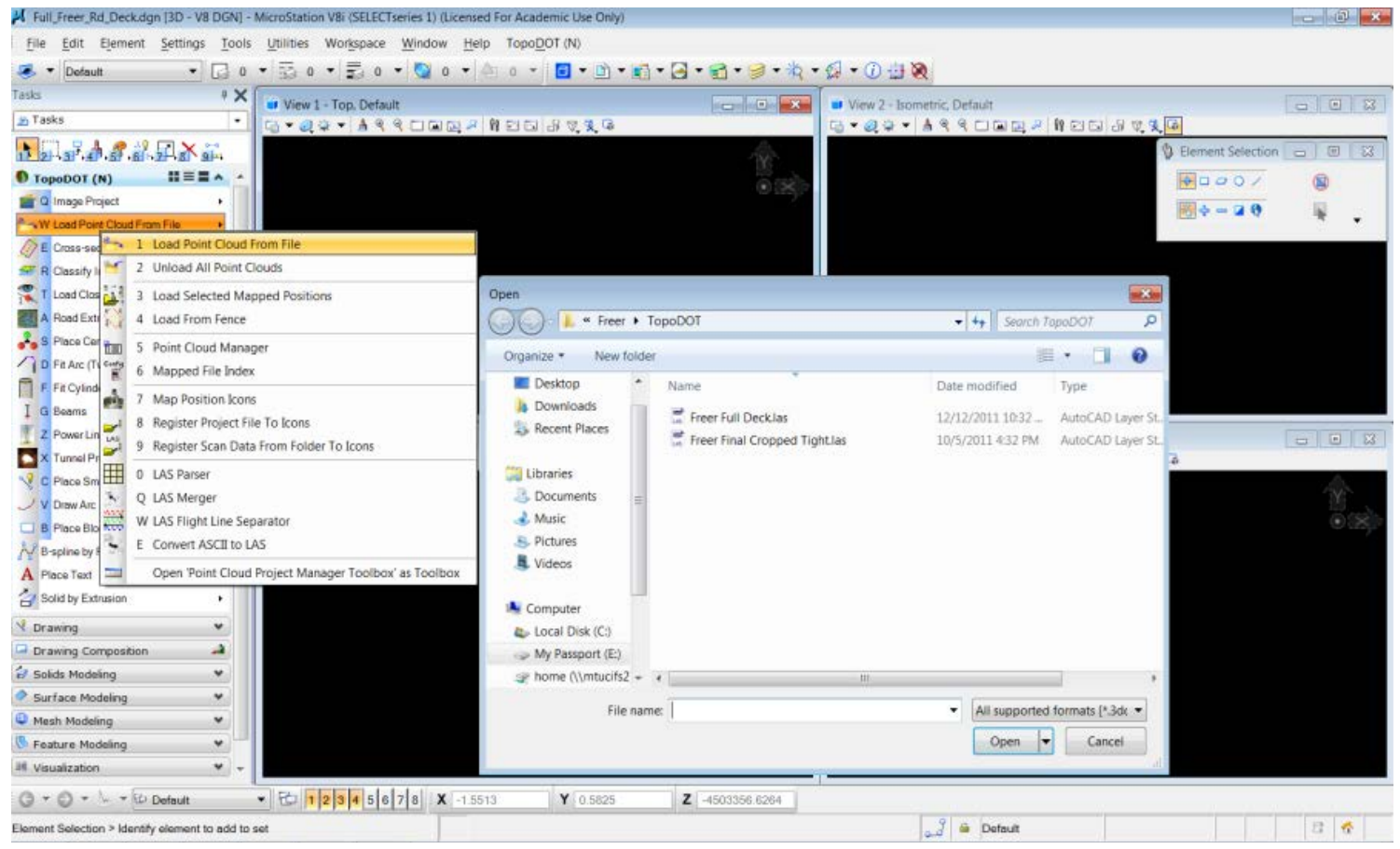

Figure 3.20: Proper procedure to load desired LAS file (Certainty 2011) (See Appendix D) 


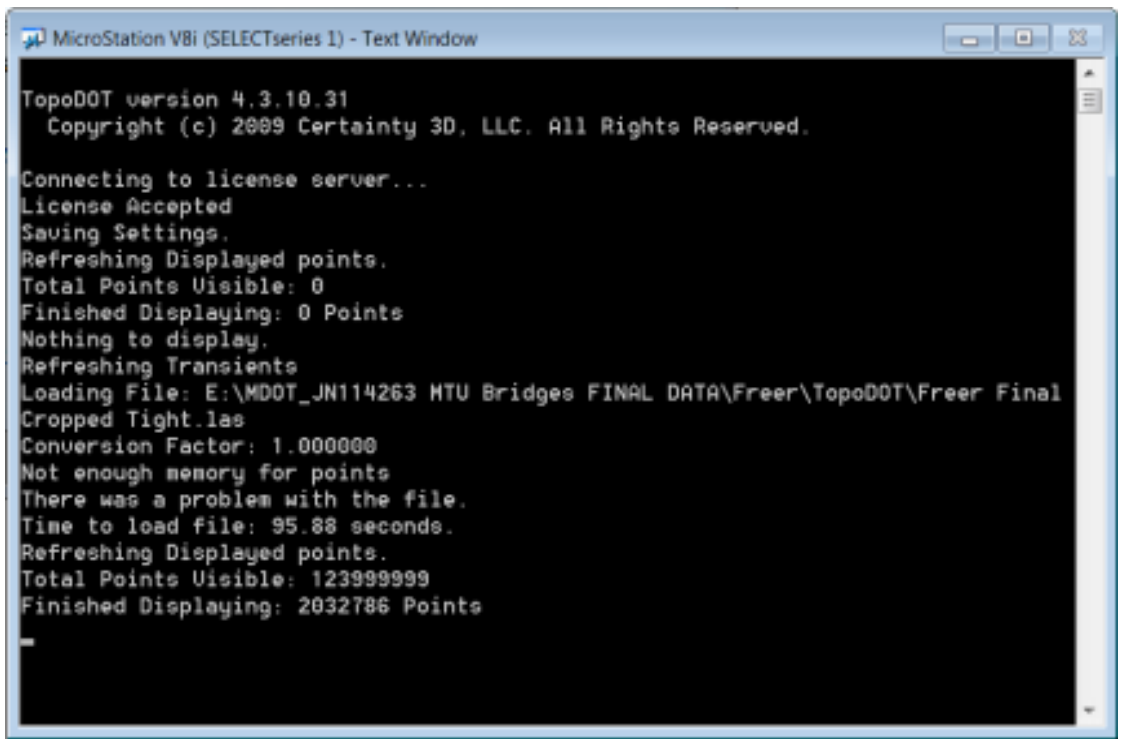

Figure 3.21: Confirming displayable points not exceeded (Certainty 2011) (See Appendix D)

After confirming that the requested point cloud data imported did not exceed the software limitations, feature extractions commenced. The TopoDOT user interface displayed the imported point cloud in four separate windows, as shown in Figure 3.22. The individual windows allowed for viewing of the data from four vantage points; top, front, right and isometric. Each window was linked to the other three allowing for real time motion tracking in all four windows.

Desired feature extraction of the reinforced concrete deck data was performed utilizing three functions, two TopoDOT and the other MicroStation defined. The two functions defined by TopoDOT used were the crop points inside and outside of the fenced area and the MicroStation function was establishing the fence. The fence tool found in the upper left hand corner was activated, displaying a "Place Fence" user prompt. The fence type was selected to be "Shape" and the fence mode remained "Inside", as shown in Figure 3.23. Using the right view window, a fence was established around the bridge deck data and the crop "Points Outside of Fence" function was used to remove the undesired points (Certainty 2011). The bridge parapets were removed from the remaining data, resulting in a planar model with residual artifacts. Residual artifacts produced from vehicles, 
pedestrians or any other disturbances were systematically removed through an iterative process of cropping.

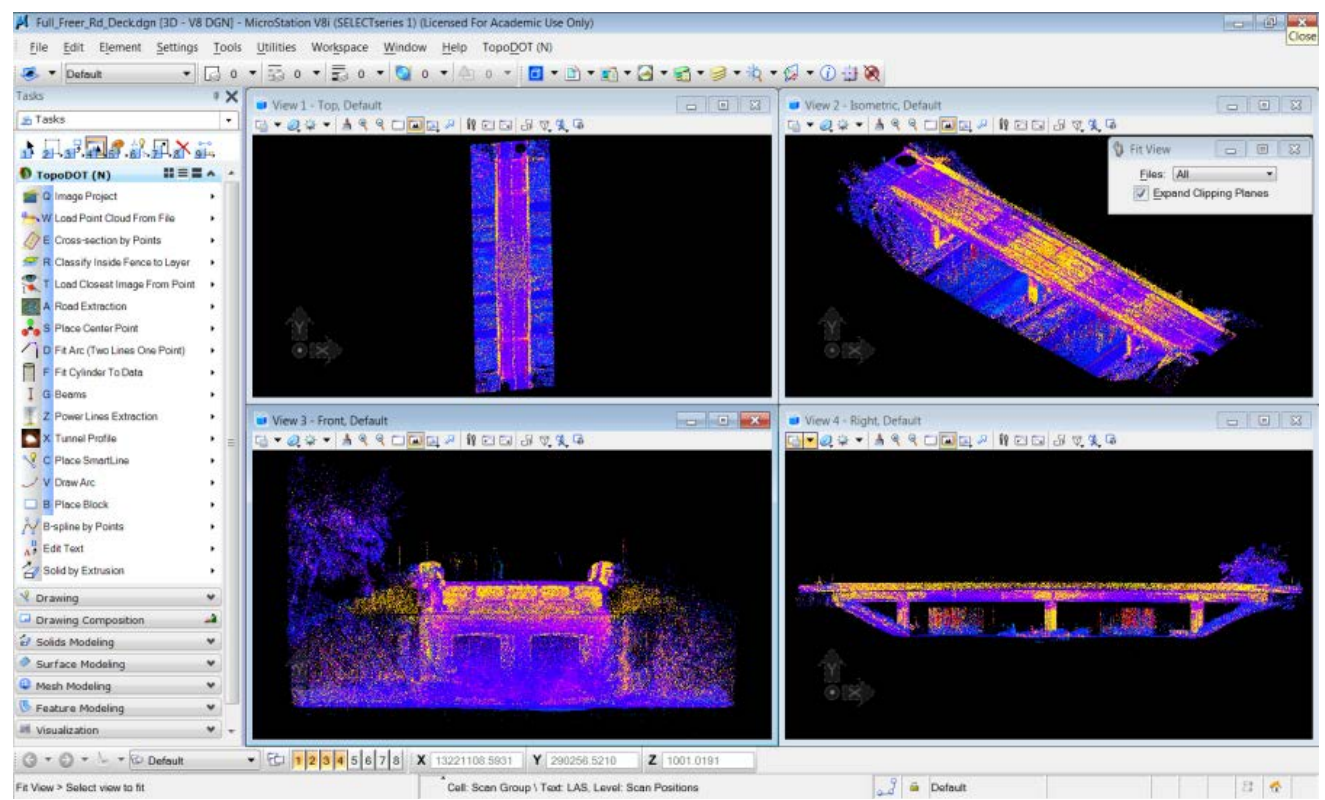

Figure 3.22: LiDAR data populated user interface (Certainty 2011) (See Appendix D)

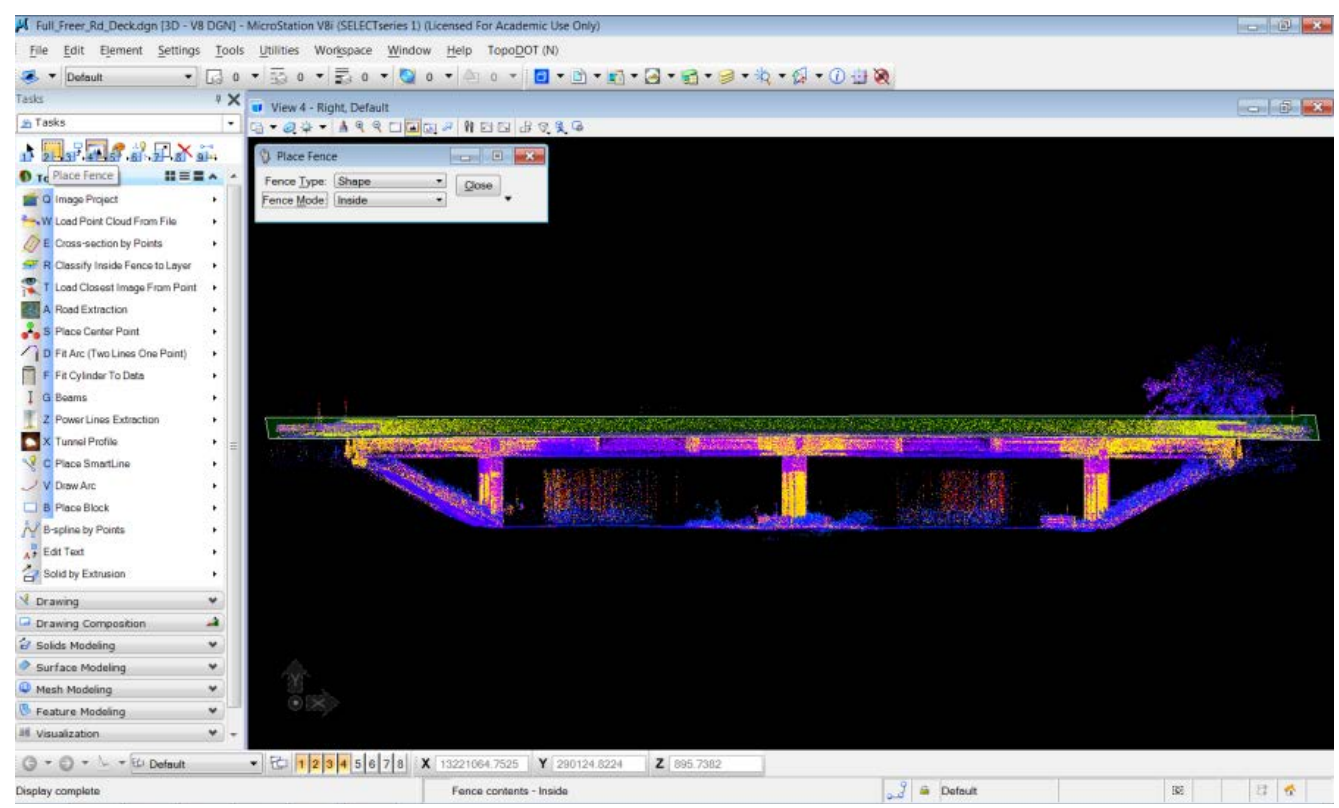

Figure 3.23: Deck extraction utilizing the fence command (Certainty 2011) (See Appendix D) 
The resulting model was a filtered data set pertaining only to the bridge deck. An isometric view of the filtered data can be seen in Figure 3.24. All feature extractions were performed in a similar manner and the resulting point clouds were exported to a separate LAS file. Exporting was completed by locating the "Export Points" function, within the "Point Cloud" icon found in the "TopoDOT (N)" drop down menu, as shown in Figure 3.25 (Certainty 2011). With the feature extractions completed, the requirement for the Certainty 3D’s TopoDOT modeling software was longer necessary.

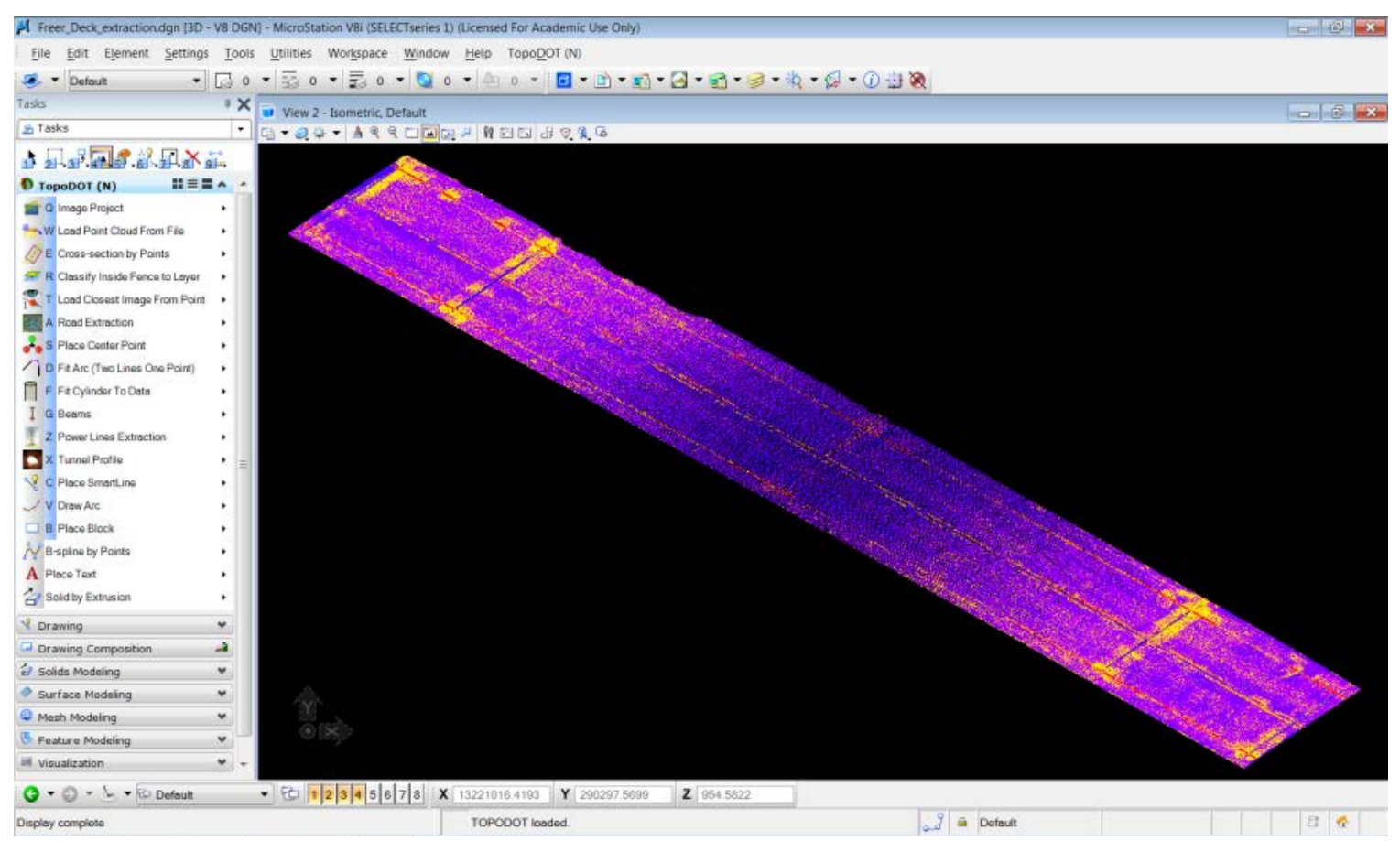

Figure 3.24: Filtered data pertaining to the bridge deck (Certainty 2011) (See Appendix D) 


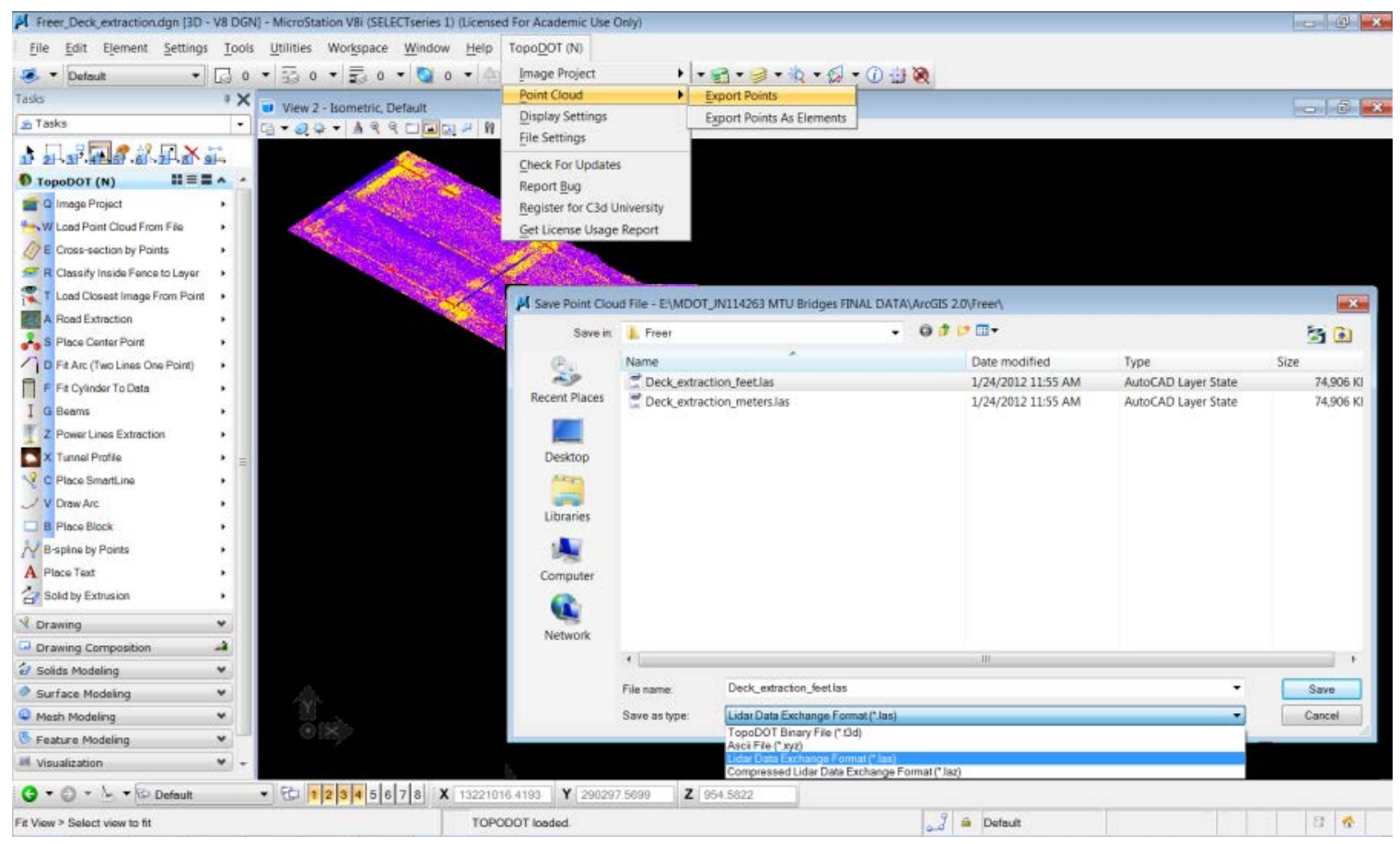

Figure 3.25: Exporting filtered deck data (Certainty 2011) (See Appendix D)

\subsection{Quick Terrain Modeler}

The requirement for the Quick Terrain Modeler software became apparent when evaluating the extracted deck data and concluded that the information needed to be divided into equivalent sections pertaining to the deck length. With terrestrial LiDAR data acquisition, as the object of interest gets further away from the receiver the relative point spacing also increasing. This increase in relative point spacing affected the ability to generate an accurate digital elevation model (DEM), because of the requirement to apply fixed point spacing to the data. The requirement to apply fixed point spacing over the entire deck data resulted in an averaging of the data, masking potential defects once the DEM was produced. Averaging of the data was completed by inserting false points in the sparsely populated areas and down sampling the points in areas of high concentration. To combat this requirement the data was further refined into eight equivalent sections according to the deck length, which was accomplished using Quick Terrain Modeler.

After loading the Quick Terrain modeling software, the user was required to load the LAS file, to be sectioned. By locating the "Import” drop down menu and selecting the 
“Import Model Data...” command, the user was requested to provide additional information prior to establishment of the correct file path to the LAS file of interest. The initial parameters were "Input Format" and "Model Format", for the required task LAS and QTA (QTC with QTA Attributes) were selected, respectively. With the initial parameters defined, the proper file was selected. Data was loaded and displayed in the user interface allowing for further data extraction, as shown in Figure 3.26 (Imagery 2011).

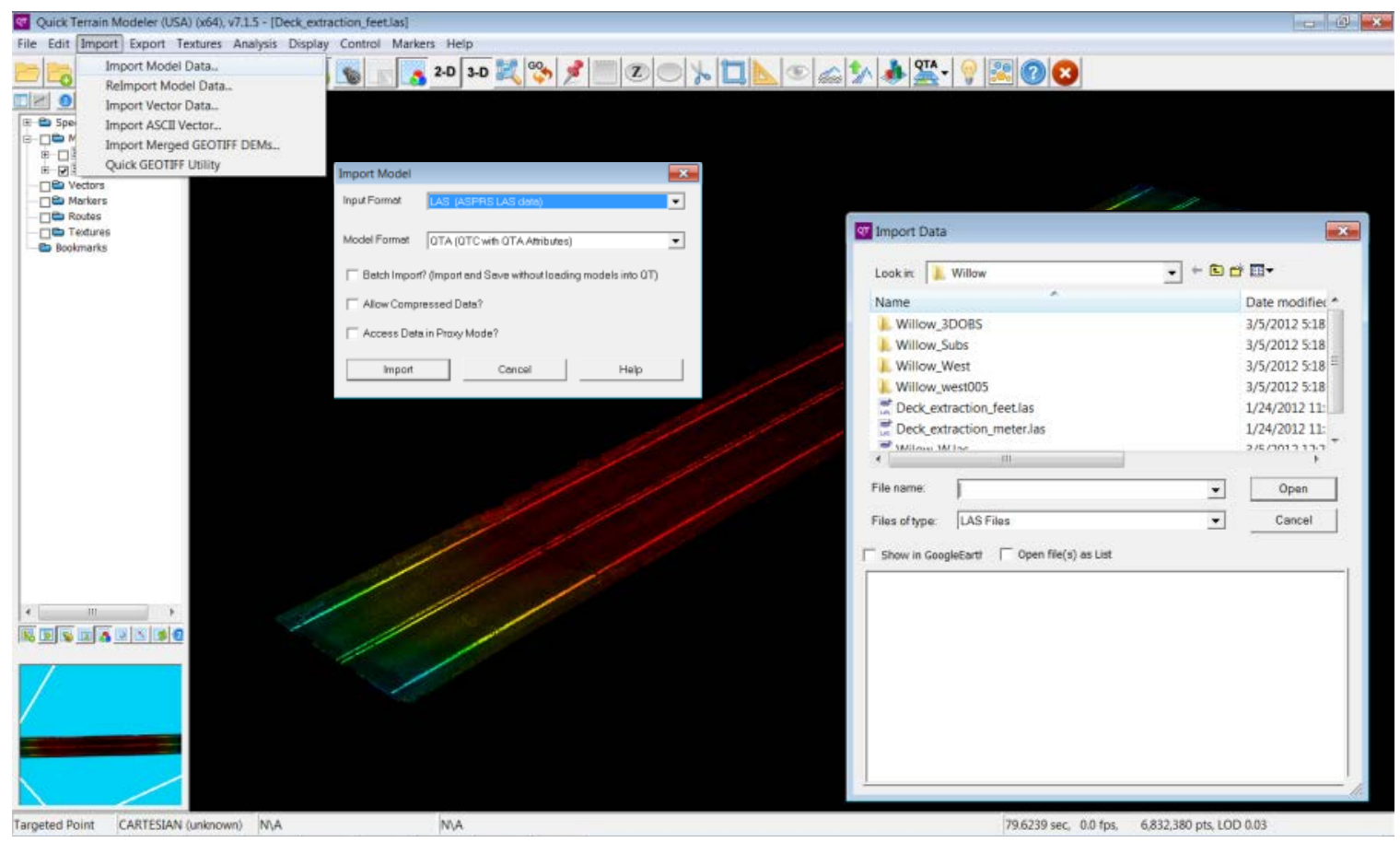

\section{Figure 3.26: Proper file import method (Imagery 2011) (See Appendix D)}

Before proceeding with the data sectioning, an appropriate naming system was established for each deck allowing for the user to readily identify the section and relative location on the deck. With each deck being sectioned into eight equal subsets, a datum was placed at the mid-span of the deck. Depending on the orientation of the bridge in reference to north, south, east and west, the first two sections from the datum were denoted by the number one and the associated bearing. For example, the Willow Road bridge is orientated east-west and is 209 feet long, so the first section to the east of the datum was denoted $1 \mathrm{E}$. 
With the file naming system defined, sectioning of the decks commenced. Sectioning was accomplished by utilizing the "Set Marker" command which was located in the "Markers" drop down menu, as shown in Figure 3.27. Each marker was set at the approximate length calculated for each desired segment length. Once the markers were placed in the approximate locations, the "Start Mensuration" function was used to refine the positioning of the marker location. The "Start Mensuration" tool was located on top toolbar, as highlighted in red on Figure 3.27 (Imagery 2011).

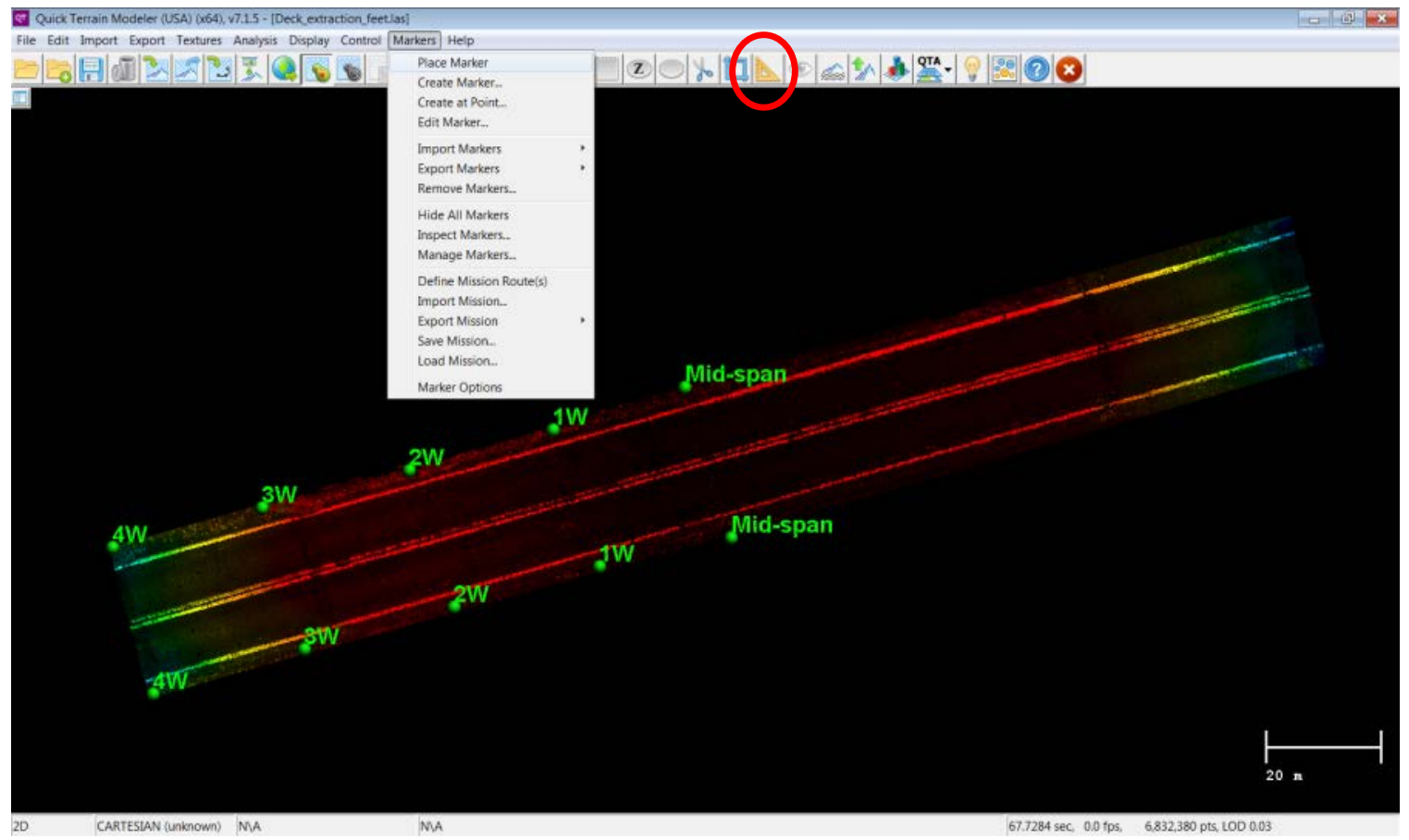

\section{Figure 3.27: Setting markers at desired section extents (Imagery 2011) (See Appendix D)}

To define the subset boundaries allowing for individual data extraction, the " $Z$ polygon" tool was used to select the extents of the subsets established by the markers, as shown in Figure 3.28. The " $Z$ polygon" tool was located on the top toolbar and is identified in Figure 3.28 by a red circle surrounding the icon. With extents selected, exporting of the selected data to a new LAS file using the established naming convention was completed. The export process was accomplished by holding down the "Ctrl” key and right-clicking on the mouse. By doing the specific routine, a command window appeared and the “Export Points” tool was selected for LAS file export, as shown in Figure 3.29 (Imagery 
2011). All the required subsets for each particular bridge were exported in a similar manner.

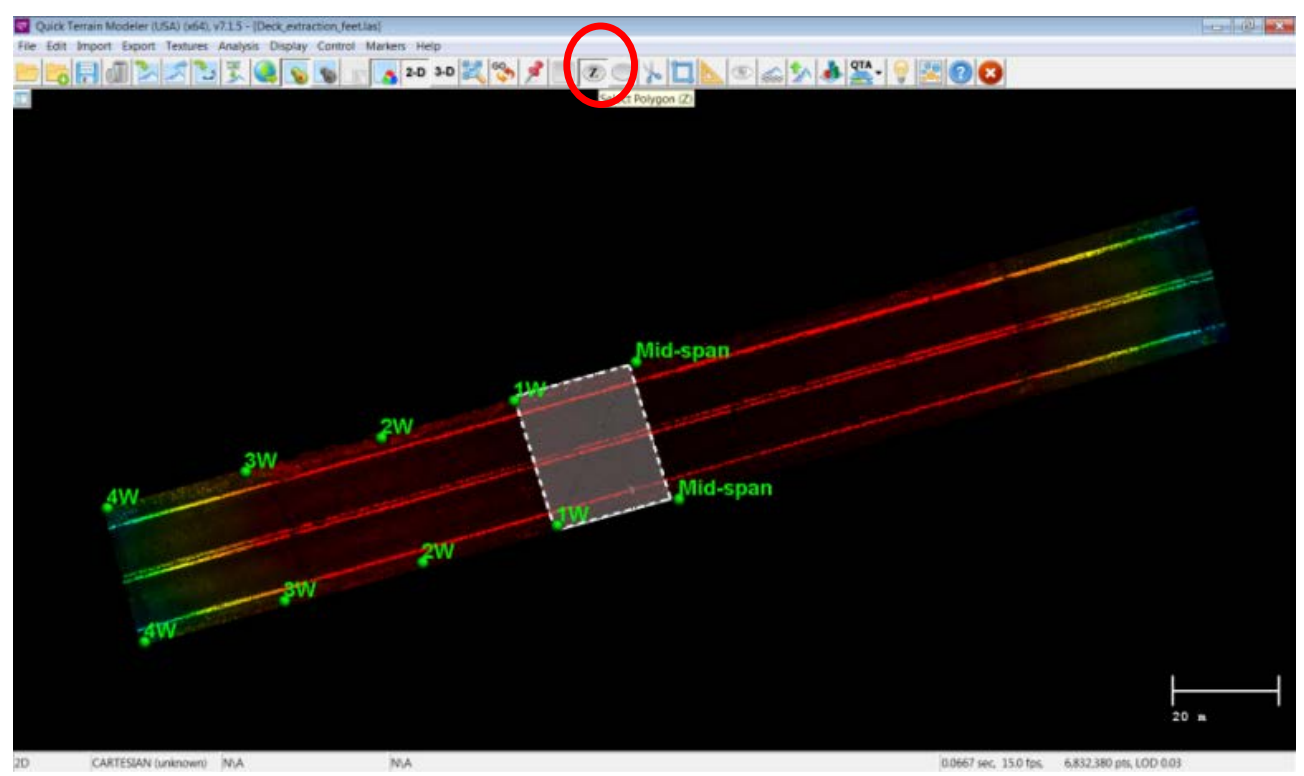

Figure 3.28: Establishing the Z-Polygon parameters for subset export (Imagery 2011) (See Appendix D)

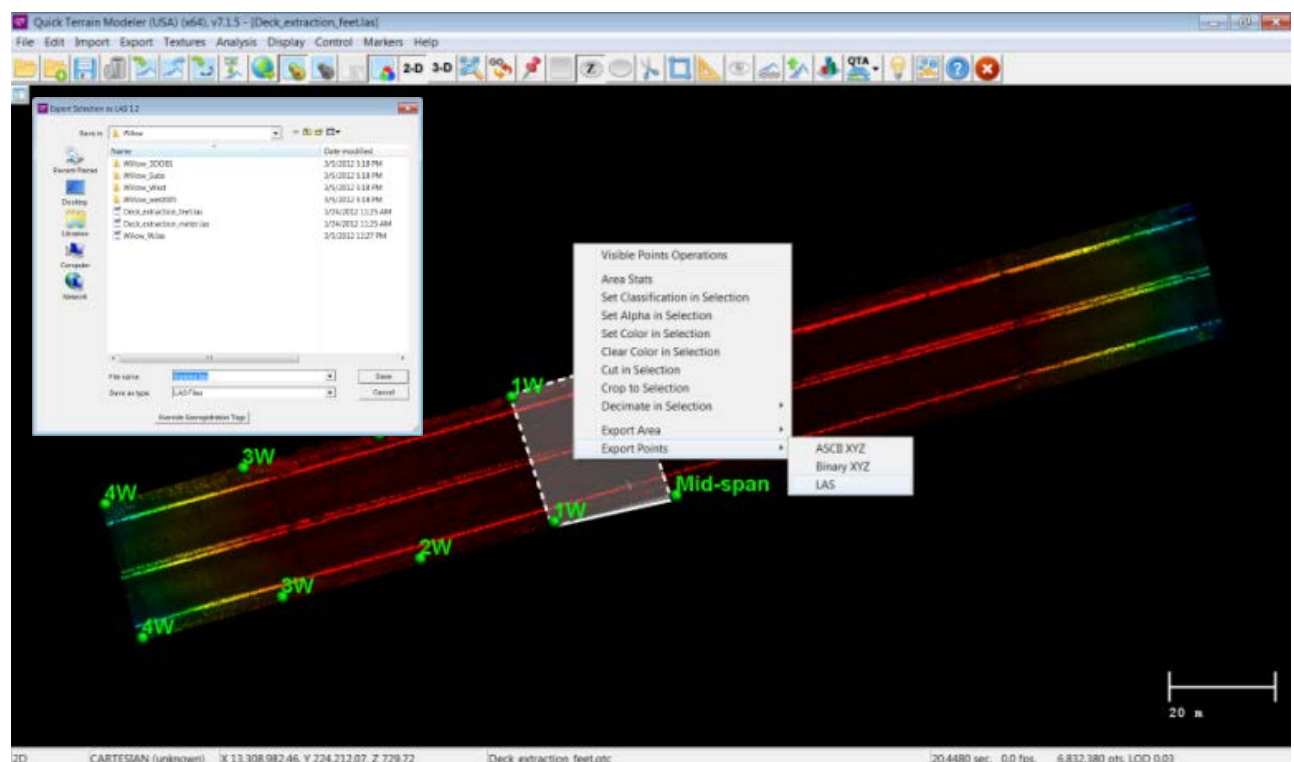

Figure 3.29: Proper exporting procedure for individual bridge subsets (Imagery 2011) (See Appendix D) 
Now that the bridge LAS files had been broken down into eight subsets pertaining to each particular bridge, the subset LAS files were converted into working geotiff DEMs. To utilize a Michigan Tech Research Institute developed algorithm for automatically detecting surface defects, the conversion from a LAS to a geotiff DEM was necessary. For the conversion, the individual subsets were required to be reimported to Quick Terrain Modeler as a QTT model. The process for importing the LAS file was similar to that depicted in Figure 3.24, with the "Model Format” changed from a QTA (QTC with QTA Attributes) to a QTT (Gridded Surface) model (Imagery 2011). When the model format was adjusted and the "Import" icon was clicked, a user prompt appeared allowing for the adjustment of the grid sampling based on point density with the subset, as shown in Figure 3.30. With being able to assign a grid sampling, the masking issue discovered during the initial post-processing phase was resolved. The default grid sampling value automatically generated within the user prompt was determine to be the average grid spacing across the entire segment multiplied by three. By simply dividing the default value by three and re-inputting the calculated grid spacing, the generated QTT model was able to be exported to a geotiff DEM without issue.

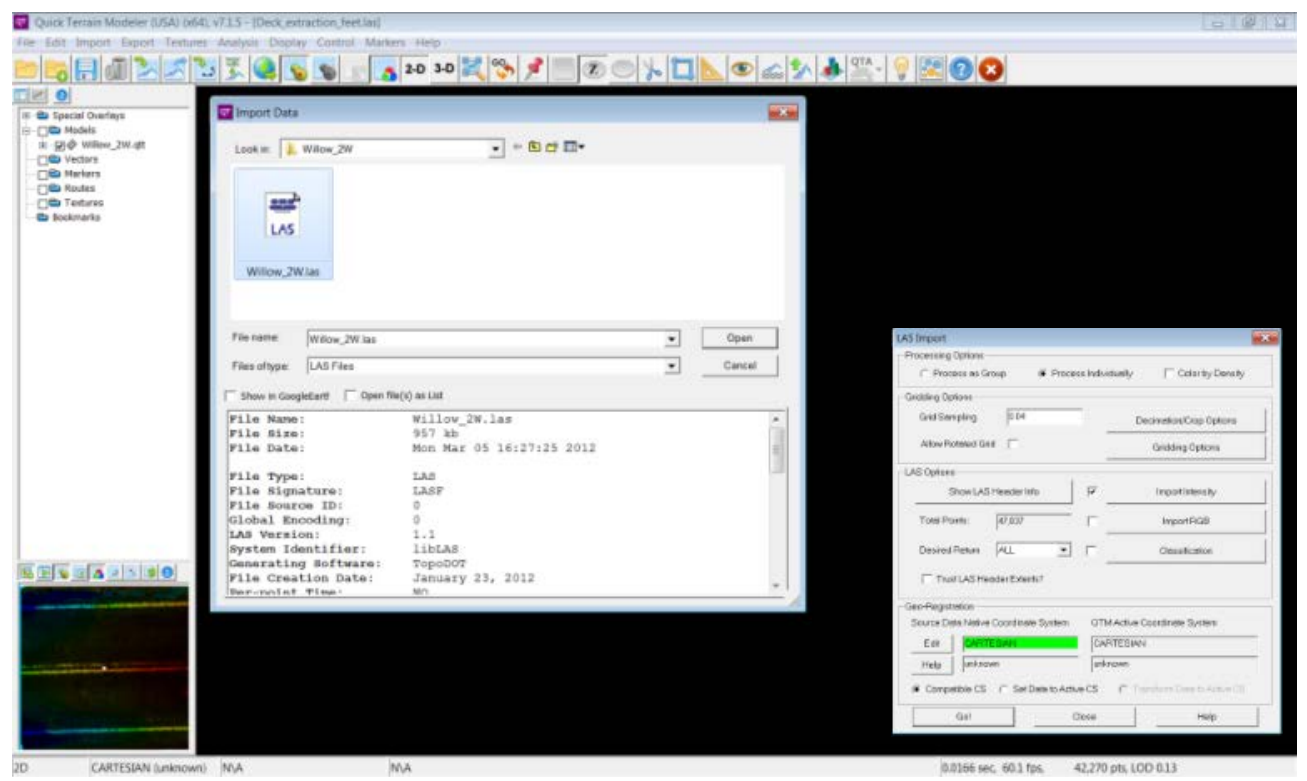

Figure 3.30: Subset file import for generation of geotiff DEM (Imagery 2011) (See Appendix D) 
To create the geotiff DEM from the QTT file of the individual deck subsets, right click on the QTT file name located in the layer table on the left hand side of the user interface, as shown in Figure 3.31. By right clicking on the desired QTT file, a dialog box appeared allowing for the user to select "Export to geotiff DEM". A user prompt appeared that allowed for the geotiff DEM to be named and saved to the appropriate directory as a TIF file (Imagery 2011). Again, the export process was completed for all the deck subsets, finalizing the data filtering process, allowing for smooth integration into the MTRI developed algorithm.

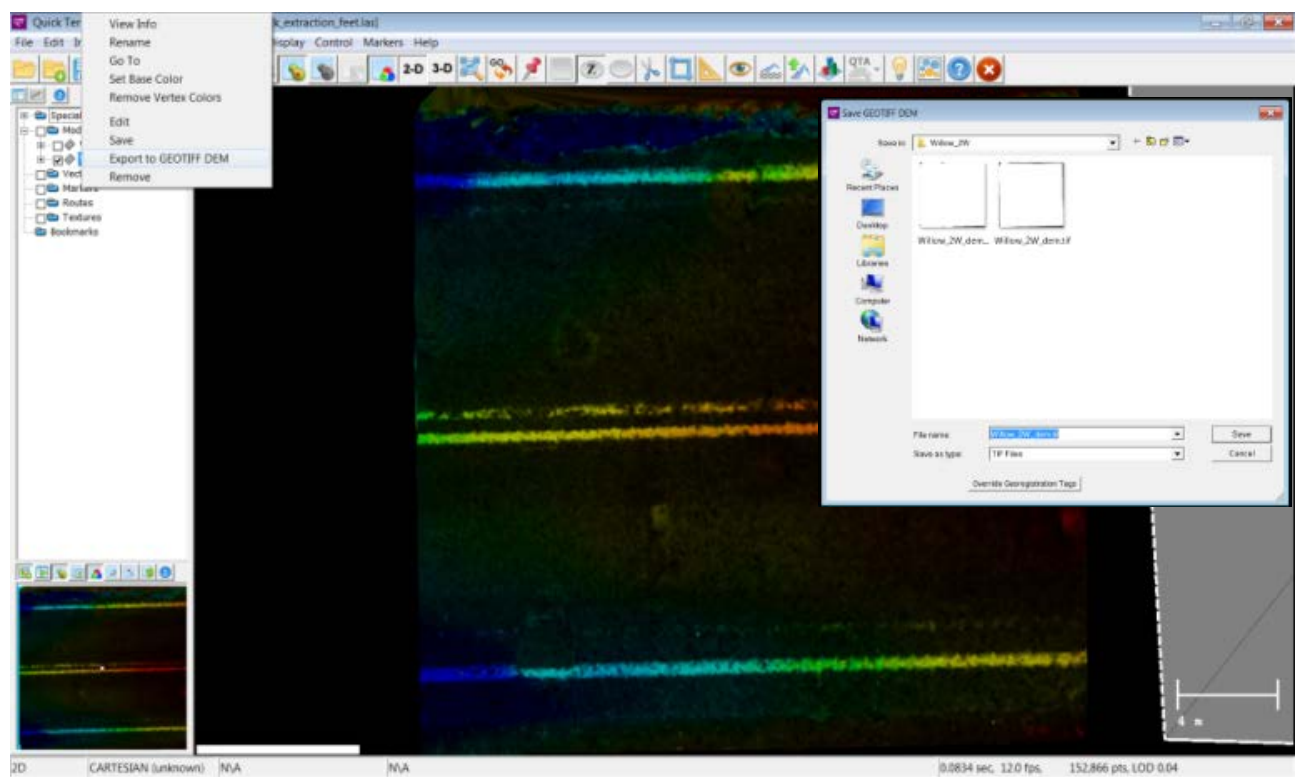

Figure 3.31: Exporting procedure for geotiff DEM file (Imagery 2011) (See Appendix D)

\subsection{MTRI 3DOBS Spall Detection Algorithm}

As part of the larger bridge condition assessment project, the Michigan Tech Research Institute (MTRI) developed algorithm, called MTRI 3DOBS spall detection algorithm, was utilized to automatically detect spalls from the bridge deck DEMs and calculate associated areas and volumes. The algorithm was written in IDLE (Python GUI), a user defined script that utilizes ESRI ArcMap geo-spatial analysis tools to evaluate inputted data (MTRI 2011). For detection and determination of defect spalls, the algorithm used a statistical method available in ESRI ArcMap, called focal statistics. Focal statistics 
evaluates individual cells by establishing boundaries around each cell referred to as "neighborhoods". Then the change in cell value is evaluated and compared to surrounding "neighborhoods". User defined parameters within the algorithm allowed for establishment of critical values defining cell value change, which would constitute a spall.

The MTRI 3DOBS spall detection algorithm evaluates elevation data, allowing for sudden elevation changes to be detected and compared with user defined spall characteristics. For proof of concept, MTRI performed analysis on an entire bridge deck 3DOBS DEM, varying the user defined minimum spall size to be considered, allowing for the establishment of minimum size criteria. The user defined minimum spall sizes evaluated were $10 \mathrm{~cm}^{2}, 100 \mathrm{~cm}^{2}$ and $1,000 \mathrm{~cm}^{2}$ allowing for a trend to be forecasted. From the testing, MTRI concluded that a minimum detection size of about $40 \mathrm{~cm}^{2}(6.2$ in $^{2}$ ) would be optimal.

With the MTRI 3DOBS spall detection algorithm developed to accept any geo-spatial DEM, the geotiff DEMS exported from Quick Terrain Modeler were valid for processing. Originally, opening the IDLE (Python GUI) program, the Python shell was the user prompt displayed. However, the MTRI developed script had to be loaded into the workspace allowing for the algorithm to be executed. When loaded, a secondary window appeared containing the MTRI developed script. To run the script, the "Run Module" function was toggled, which was found in the "Run" drop down menu.

By selecting to run the module, the Python shell user prompt was populated with multiple system required inputs, as shown in Figure 3.32 (MTRI 2011). Six user defined parameters were required to be inputted in the Python shell user prompt. Important to note is that when inputting the working directory path, bridge joint file name and DEM name, the information provided in the user prompt must match exactly to the actual. If any character was incorrectly defined the algorithm would fail. 


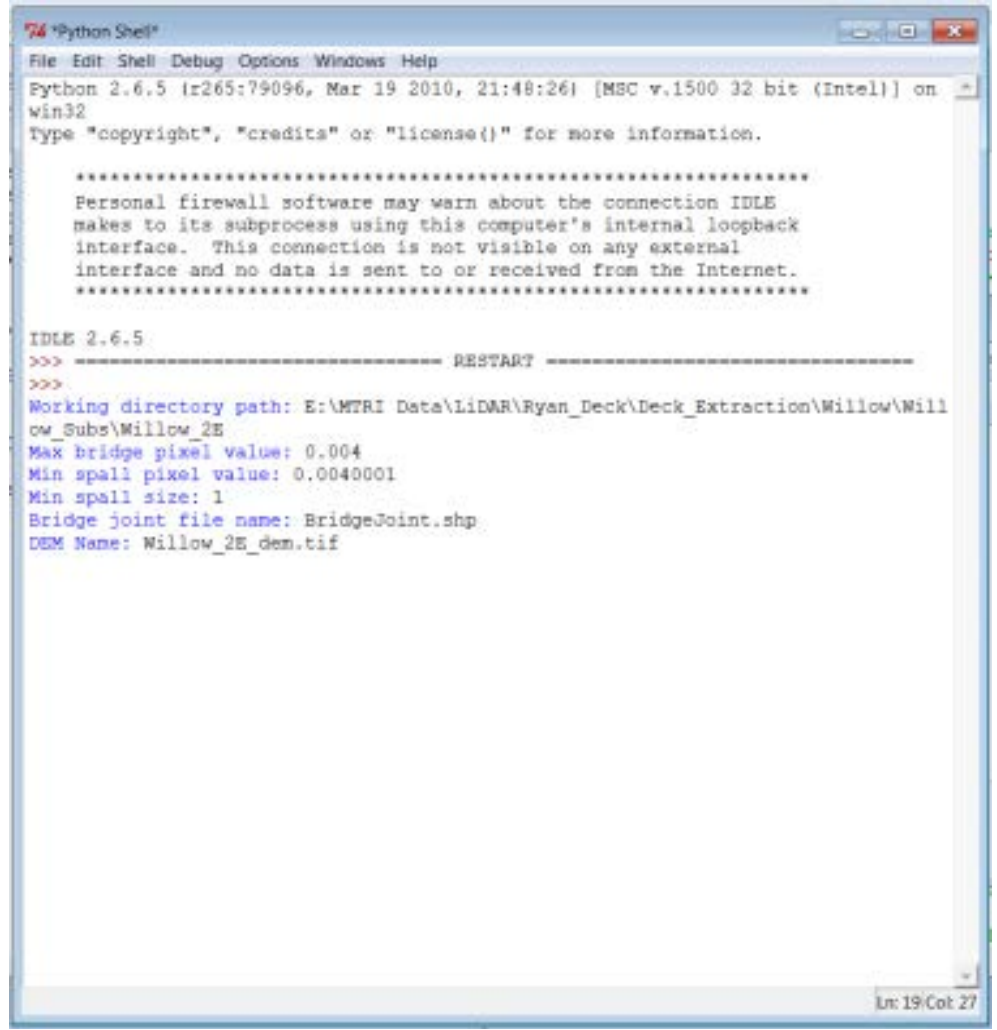

Figure 3.32: MTRI 3DOBS spall detection algorithm user prompt (MTRI 2011)

Additionally, spall detection parameters were established within the Python shell user prompt. The max bridge pixel value requested during this process allowed for the user to define the maximum allowable standard deviation value that would be considered undamaged concrete. Once the defined standard deviation value was exceeded, that pixel pertaining to that exceeded standard deviation value was classified as a spall, as defined by the min spall pixel value. With a spall typically being defined as a volume of removed material caused from various concrete deterioration mechanisms, surface area also was required to be defined. The surface area parameter was inputted during the min spall size request, which established a pixel based minimum area, which was required to be met or exceeded to be classified as a spall.

With all the user defined parameters established, the algorithm ran automatically, populating the recognized working directory with outputted ESRI ArcMap files and a Microsoft Excel spreadsheet, as shown in Figure 3.33. The Microsoft Excel spreadsheet 
contained volume and area calculations pertaining to all detect surface defects. Multiple iterations of the algorithm were performed on each section of the individual bridge decks allowing for generation of not only the Microsoft Excel spreadsheet, but a geo-reference set of visual aids, as seen in Figure 3.34. These visual aids allowed for the user to verify that the results generated from the algorithm aligned with visible surface defects present on a high-resolution optical image.

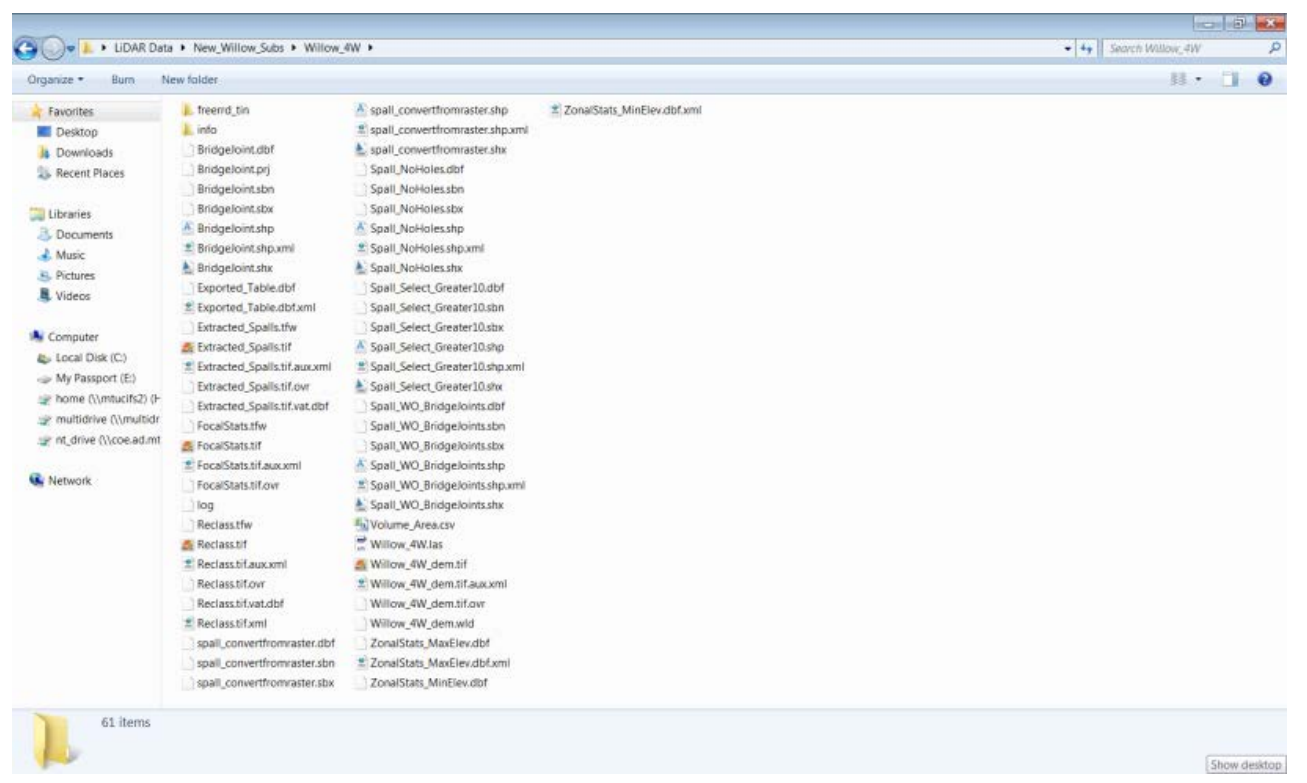

Figure 3.33: Populated working directory from MTRI 3DOBS spall detection algorithm (MTRI 2011)

Refined analysis was performed on areas of known spall volume and compared with a secondary surface defect detection technology to validate results. With all processes, meticulous preparation and forethought was necessary to produce the desired result. Contained within the following sections are the results and discussion produced from the described methodology. 


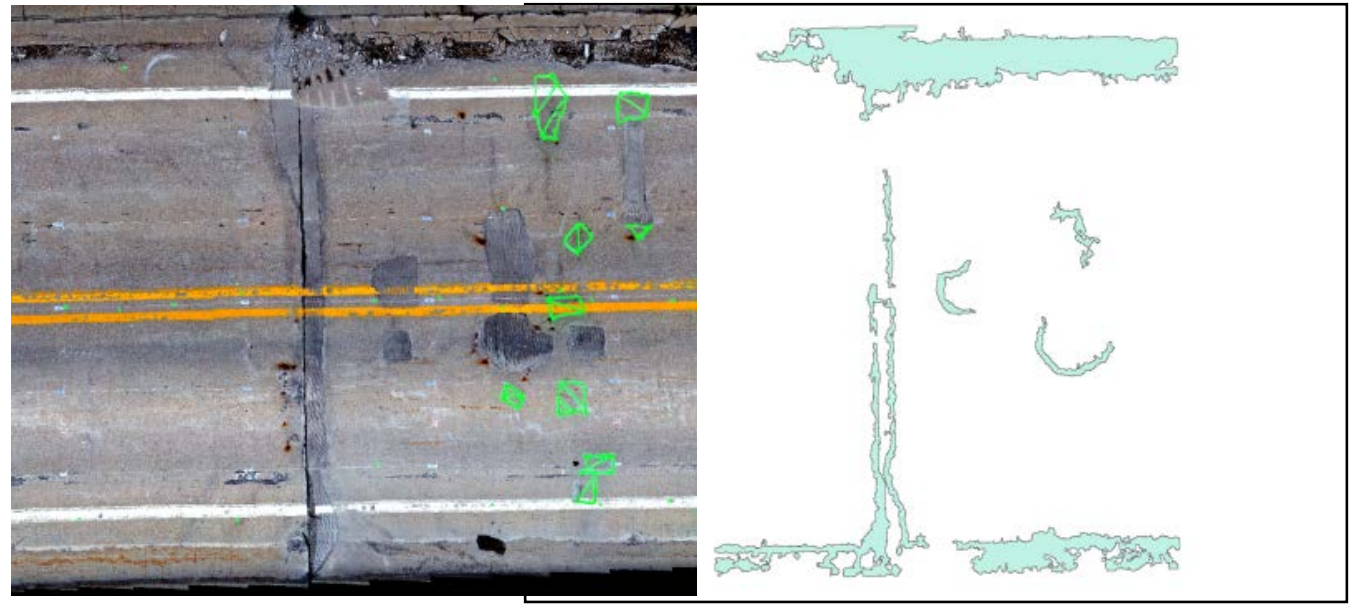

Figure 3.34: Optical visual aid for validation of spall detection process (MTRI 2011) 


\section{Results/Discussion}

With the filtered deck LiDAR pertaining to the individual field demonstration locations evaluated utilizing the MTRI 3DOBS spall detection algorithm, results are presented. Principal findings are summarized. Further investigation to the individual field demonstration results are provided along with specific feature extraction of test spall locations allowing for comparison of findings to the applied ground truth measure.

\subsection{General Findings from Full-scale Field Demonstration}

\subsubsection{Point Cloud Density Decay}

During the field demonstration, the number of bridge deck data acquisition locations was held constant; the two locations were typically on the bridge approaches on either abutment. The resolution of the individual scans were held constant at the highest achievable resolution of 50,000 points/second, except for the Mannsiding Road north bound (NB) overpass, which was collected at a medium resolution rate due to time constraints. Unfortunately, the specific resolution of the Mannsiding Road NB overpass data collect was not provided. Even with the variation in the rate of data collection, a reoccurring theme became evident during the data post-processing of all four bridge decks, point cloud density decay.

The findings showed that the point cloud density decayed at an exponential rate rather than the anticipated linear decay referenced in section 2.9. The theory developed by the Advanced Highway Maintenance and Construction Technology (AHMCT) Research Center at the University of California, Davis was for a spherical object, which presented a constant surface area for detection. The planar bridge deck surface evaluated in this research produced a diminishing coverage angle resulting in non-linear point cloud decay. Figures 4.1 through 4.8 visually and graphically show the exponential point cloud decay of the LiDAR data as the distance from the receiver is increased.

The Figures 4.1, 4.3, 4.5 and 4.7 visually demonstrate the exponential point cloud decay. Areas represented in red are locations near collection points and the blue represents the 
lowest point density captured on the bridge deck surface. The densest recorded reading for all four bridge decks was 7,736.3 points per $\mathrm{ft}^{2}\left(\mathrm{ppft}^{2}\right)\left(83,186\right.$ point per $\mathrm{m}^{2}\left(\mathrm{ppm}^{2}\right)$ ), while the least populated section was $18 \mathrm{ppft}^{2}\left(193.5 \mathrm{ppm}^{2}\right)$. The remaining figures show a longitudinal elevation profile of the bridge decks and as the sensed data points approach the middle bridge span of each deck, the red line transitions to a dashed line. The transition of the line type is due to the point cloud density decay and the increase in the relative distance between each point, the white space between the red dashes is assumed to be a hole by the software. 


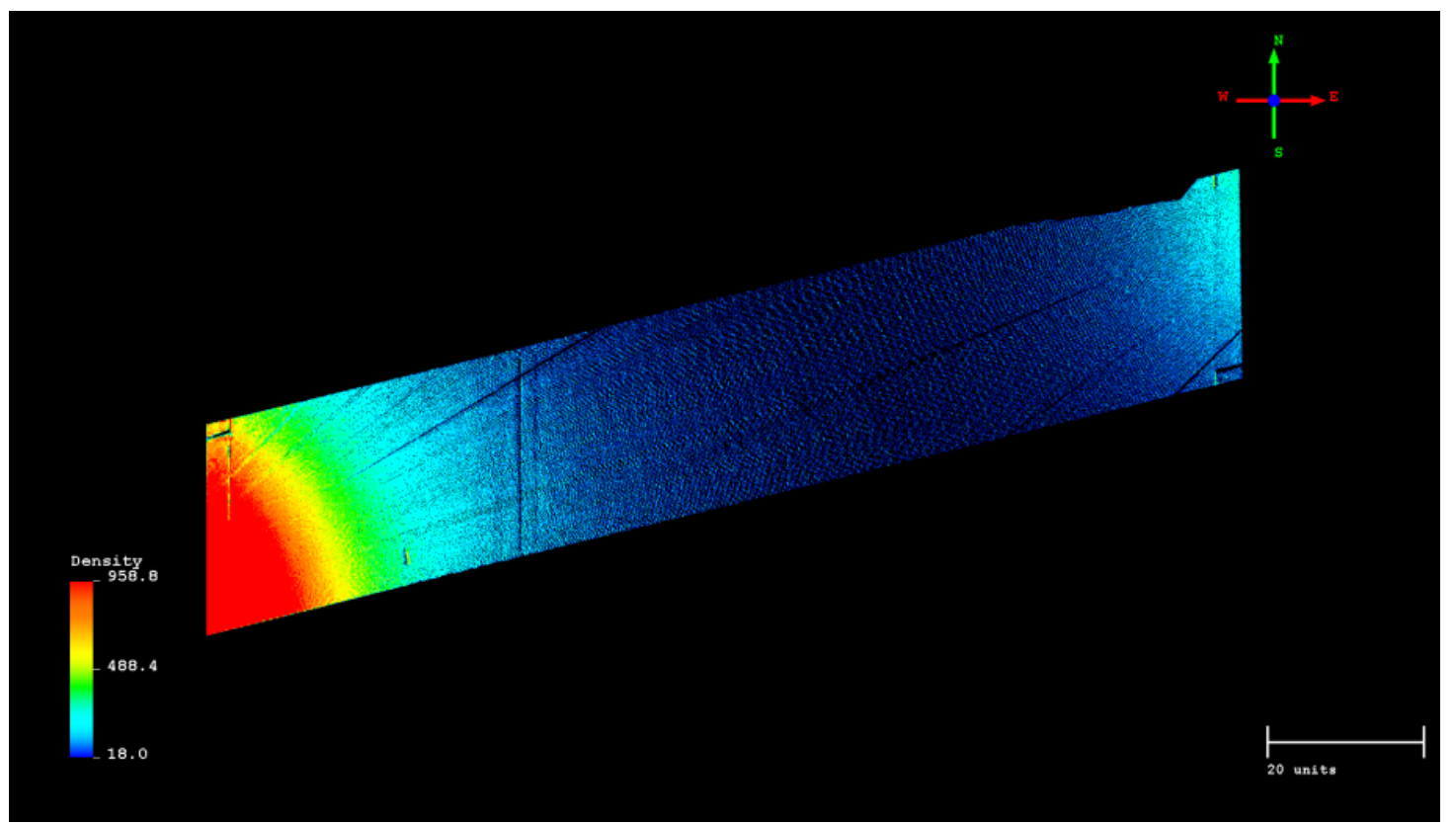

Figure 4.1: Mannsiding Road NB point density texture for visual representation

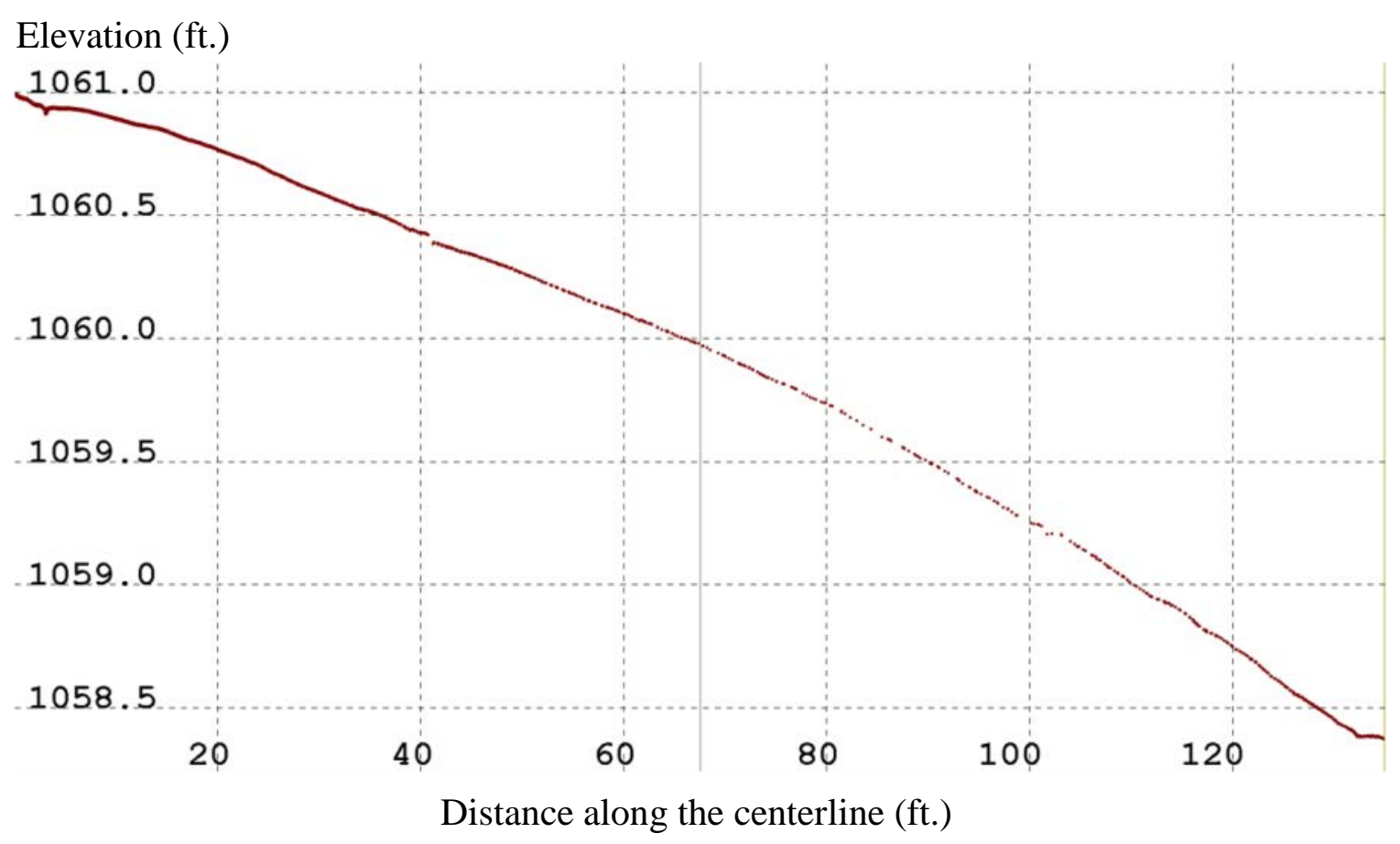

Figure 4.2: Mannsiding Road NB longitudinal elevation profile 


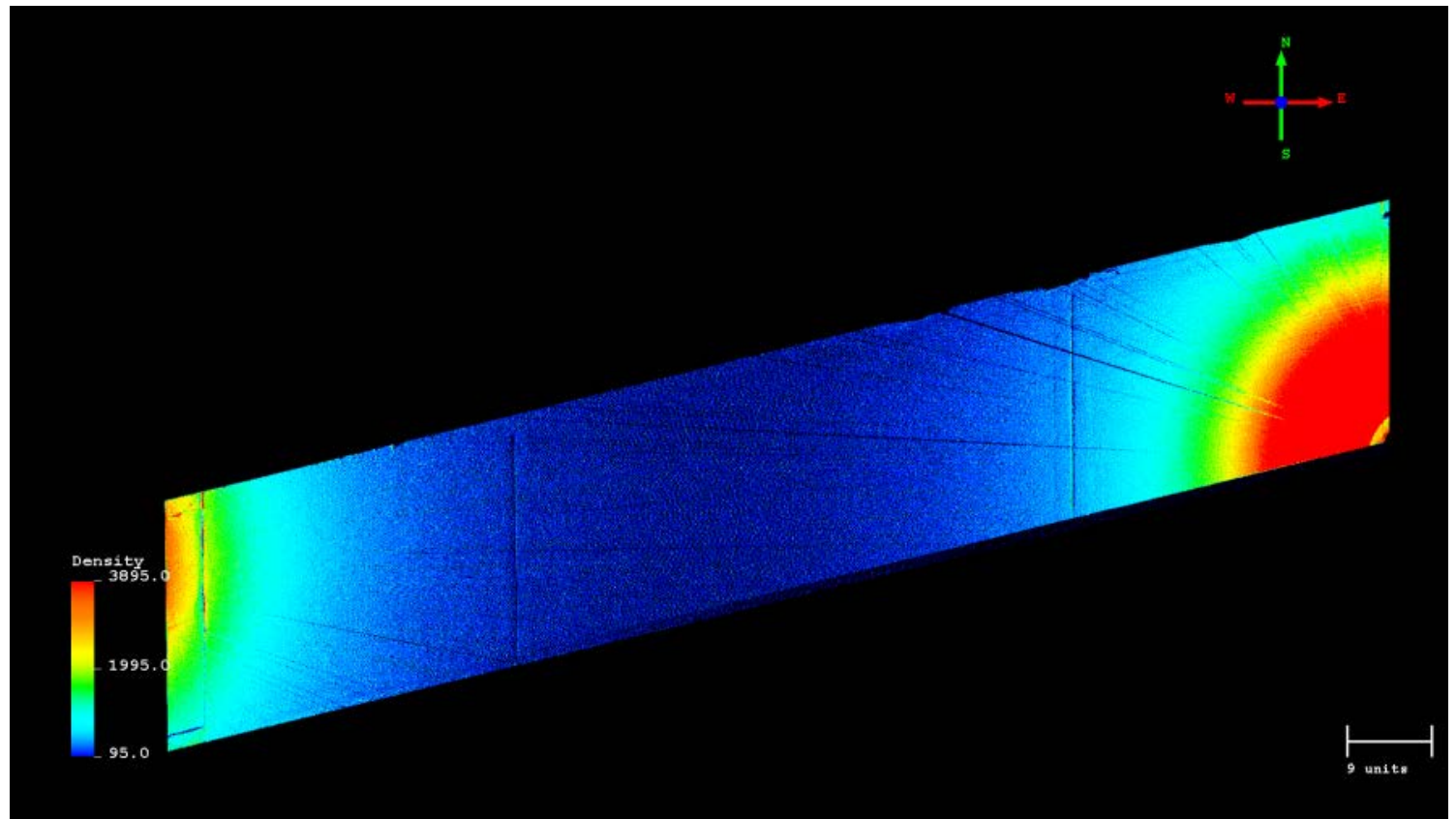

Figure 4.3: Mannsiding Road SB point density texture for visual representation

Elevation (ft.)

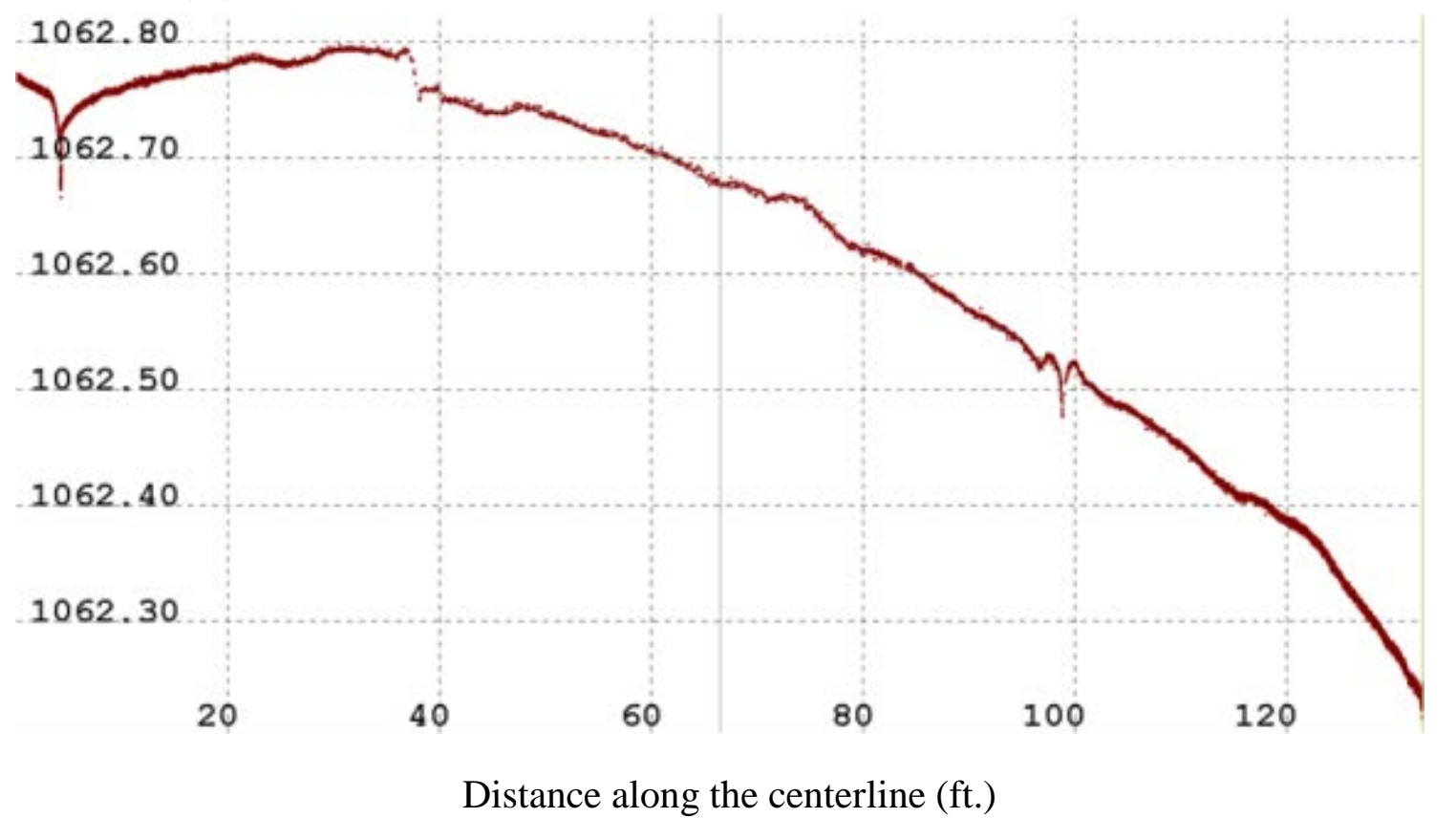

Figure 4.4: Mannsiding Road SB longitudinal elevation profile 


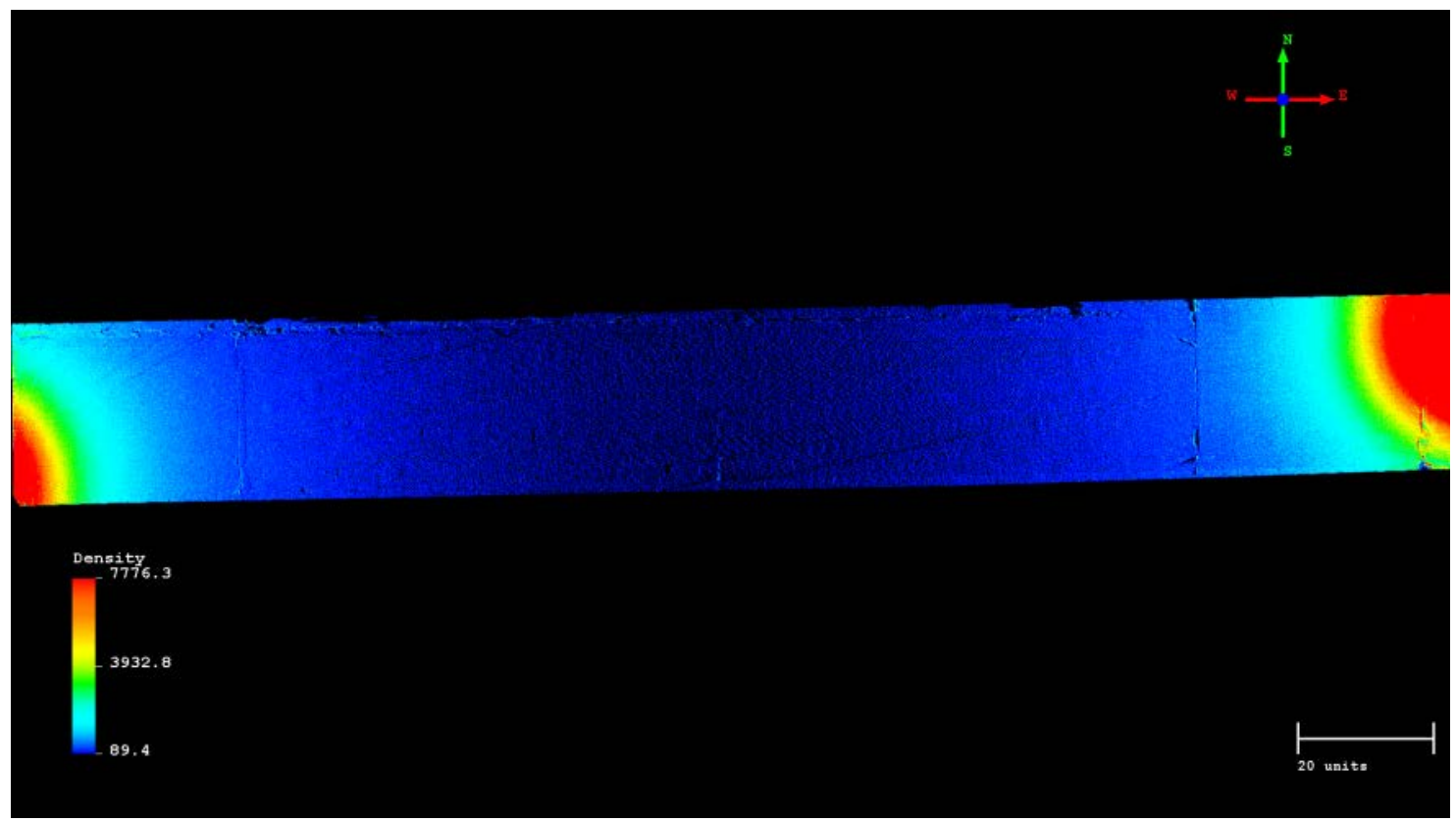

Figure 4.5: Willow Road point density texture for visual representation

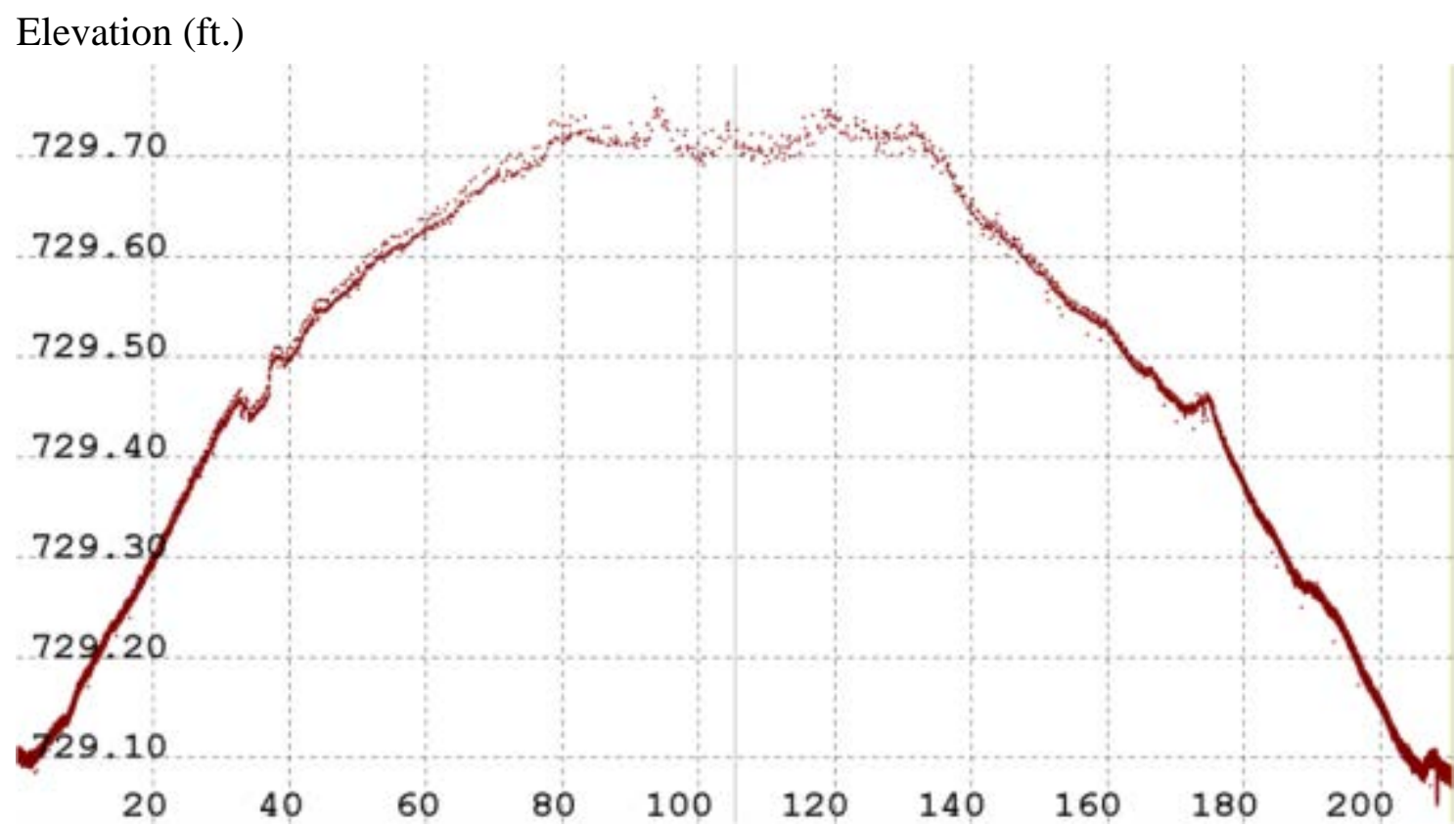

Distance along the centerline (ft.)

Figure 4.6: Willow Road longitudinal elevation profile 


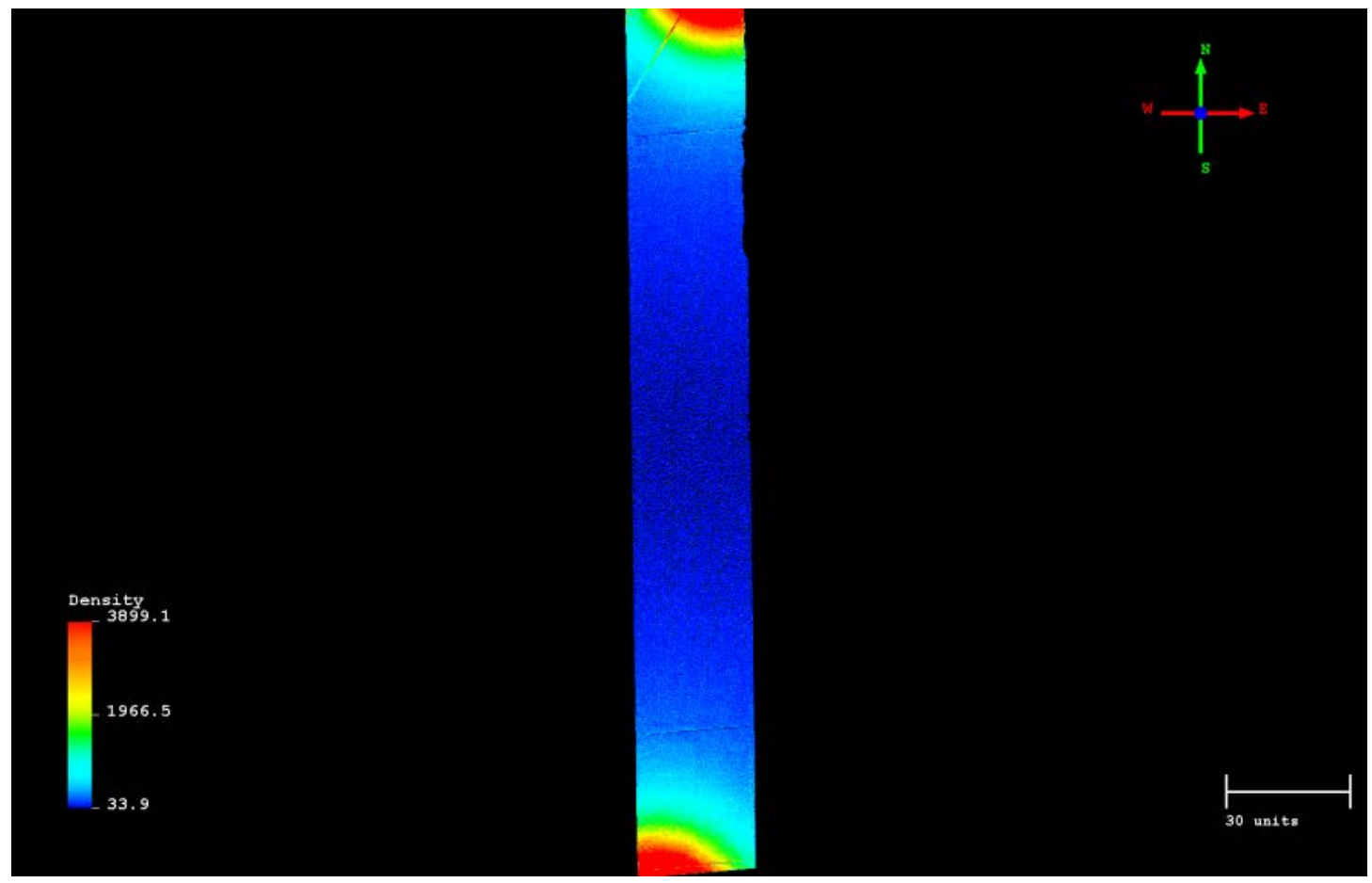

Figure 4.7: Freer Road point density texture for visual representation

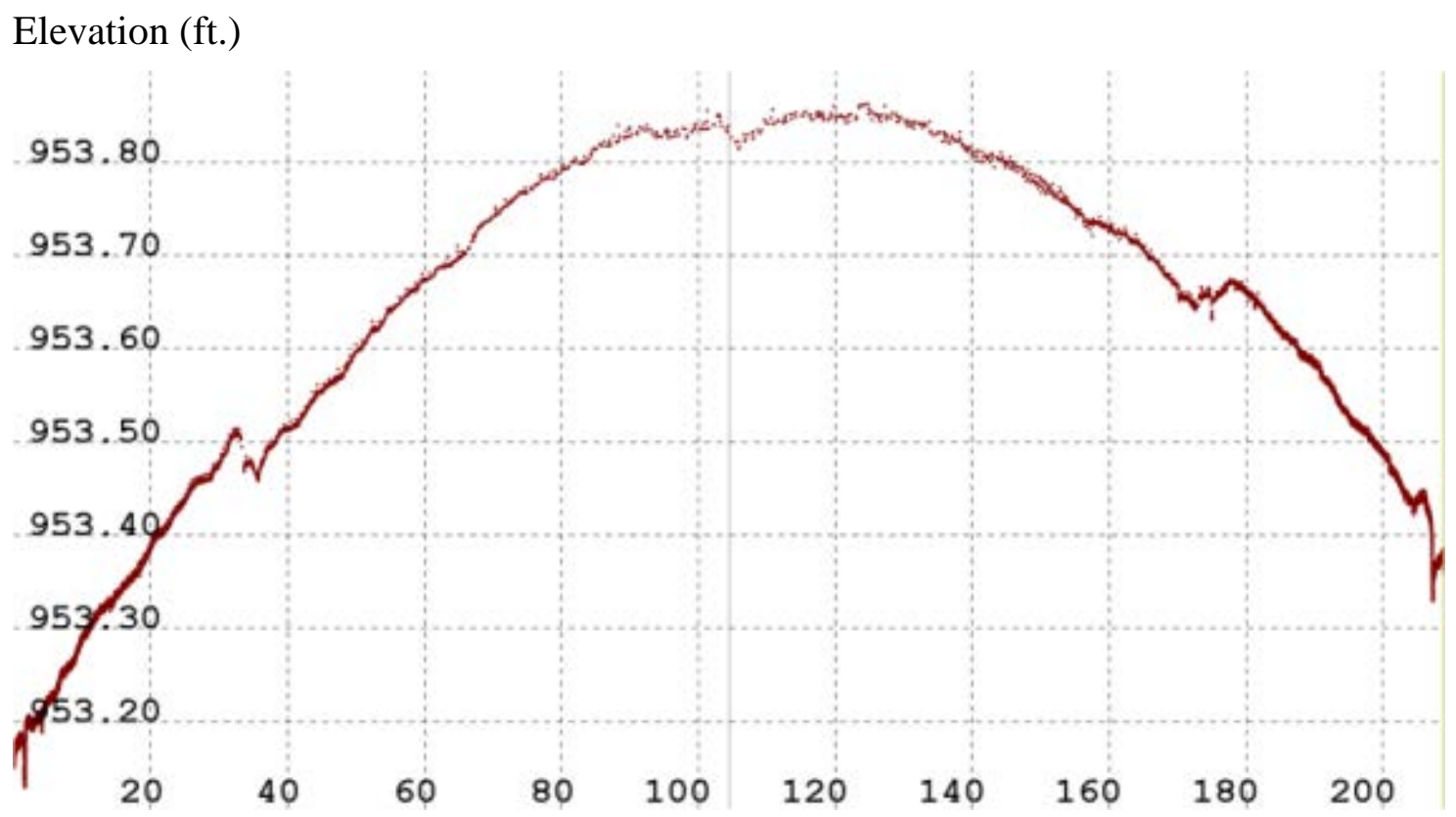

Distance along the centerline (ft.)

Figure 4.8: Freer Road ıngıtuainaı eıevation prome 
To explain the unexpected exponential point cloud decay, collection and processing methods were reevaluated. By relying on two stationary collection locations at a fixed collection height, the angle of incidence greatly influence the obtainable surface information. Figure 4.9 and Figure 4.10 show that as the distance from the receiver increased, the angle of incidence initially increased rapidly and asymptotically approached 180 degrees. The established relationship for the angle of incidence resulted in an equal reduction in the coverage angle. When sensing a planar surface for topographical defects, a coverage angle of nearly 180 degrees would be ideal. However, with terrestrial LiDAR units, the coverage angle is substantially less than the desired 180 degrees without an elevated platform.

This reduction in coverage angle is only enhanced with the presence of the built-in bridge arch. As shown in the longitudinal elevation profile graphs, the arches of the bridges were captured during the data acquisition. The maximum arch present in the four test cases was estimated to be $0.8 \mathrm{ft}$ of elevation change (on Freer Road from abutment to the middle span).

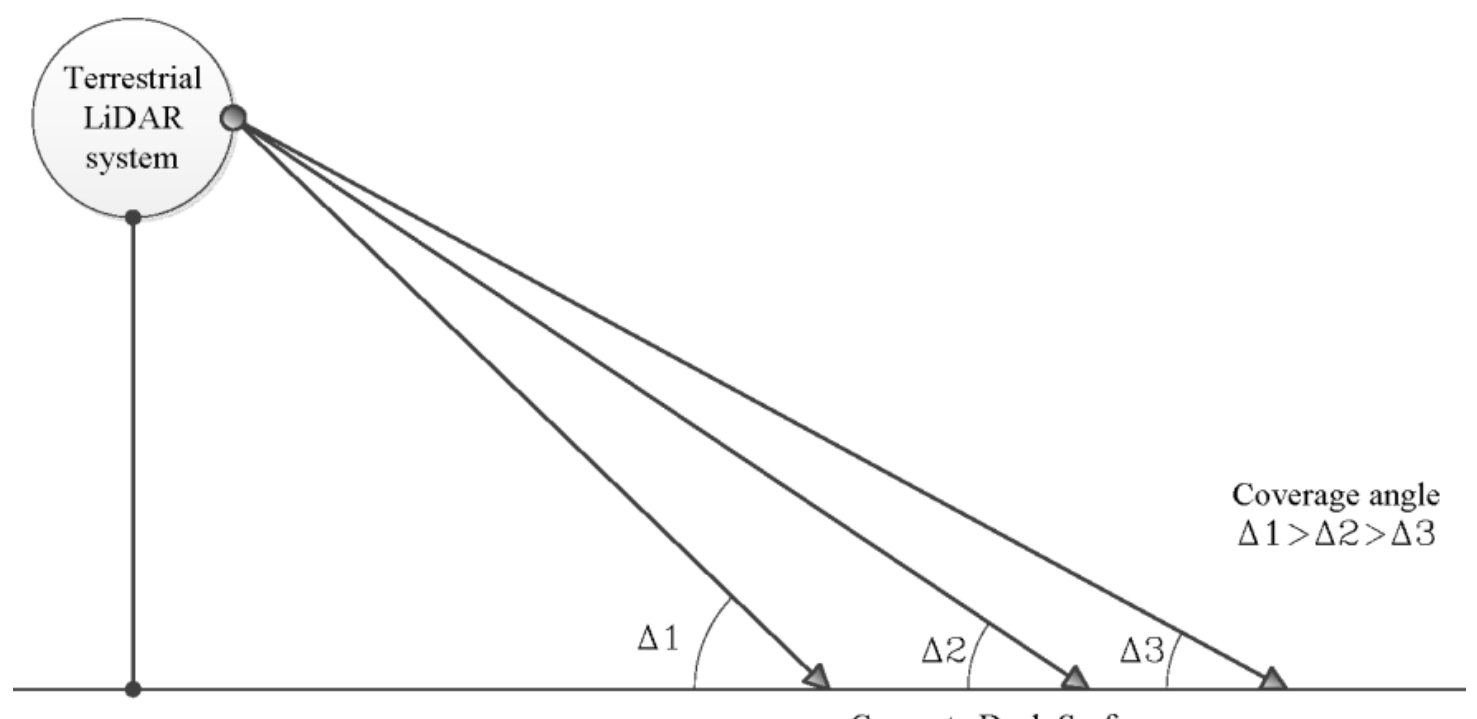

Concrete Deck Surface

Figure 4.9: Visual representation of the reduction in coverage angle over distance 


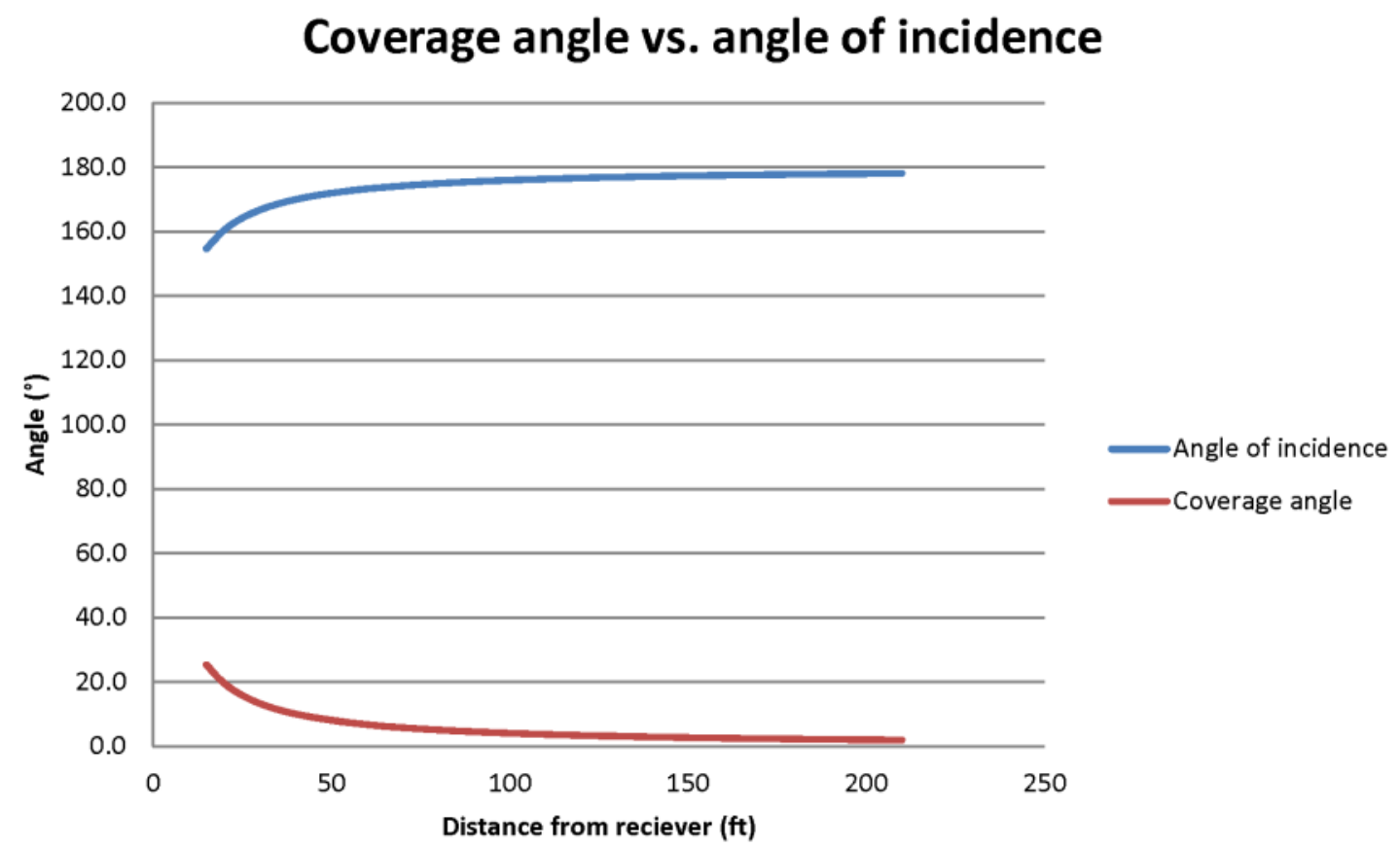

Figure 4.10: The coverage angle and angle of incidence as the distance varies

\subsubsection{Maximum Radius of Capture}

When evaluating the MTRI 3DOBS spall detection algorithm results, the estimated spalled area increased as the distance from the terrestrial LiDAR unit increased. The cause of the increasing overestimation of spalled area was attributed to the exponential point cloud density decay rate. By comparing the optical master deck images of each case study bridge and the algorithm results, a maximum radius of data capture was generated for the Leica ScanStation C10 at the highest collection rate, 50,000 points/second. In Appendix C, the algorithm results are provided to view the estimated surface defects. By measuring the distance from the edge of the approach slab to the point of error saturation, a maximum radius of capture trend was established. The individual bridge measurements for maximum radius of capture are listed in Table 4.1. 
Table 4.1: Maximum radius of capture for Leica ScanStation C10 at 50,000 points/second

\begin{tabular}{|c|c|c|}
\hline Field Demonstration Location & Approach Slab & Radius of Capture (ft) \\
\hline \multirow{2}{*}{ Mannsiding Road NB } & Northeast & 0.00 \\
\cline { 2 - 3 } & Southwest & 50.78 \\
\hline \multirow{2}{*}{ Mannsiding Road SB } & Northeast & 66.48 \\
\cline { 2 - 3 } & Southwest & 66.48 \\
\hline \multirow{2}{*}{ Freer Road } & North & 51.59 \\
\cline { 2 - 3 } & South & 52.09 \\
\hline \multirow{2}{*}{ Willow Road } & East & 50.76 \\
\cline { 2 - 3 } & West & 55.37 \\
\hline
\end{tabular}

The average radius of capture, when considering all terrestrial LiDAR locations where a high resolution scan was collected, was $56.2 \mathrm{ft}$. The established radius of collection of $56.2 \mathrm{ft}$ only pertains to the case study collection parameters.

\subsection{Full-scale Field Demonstration}

With the understanding of the exponential point cloud decay and the maximum capture radius, individual evaluation of the bridge decks was conducted. To reinforce the two general findings, full deck evaluations for surface defects were conducted; results are provided and compared with an established ground truth measure. The full deck evaluations were only performed on data within the established 56.2 foot radius, data outside the radius was not appropriate for evaluation, because of point cloud decay. For the algorithm to successfully process the eight subsets for each bridge, constant spall parameters were established. The constant spall parameters were as follows:

- Max bridge pixel standard deviation: 0.004

- Min spall pixel standard deviation: 0.0040001

- $\quad$ Min spall pixel size: 1.0

Known surface defects located within the established 56.2 foot coverage radius were further evaluated allowing for a determination of the effectiveness of the technology, data collection and processing methods under ideal conditions. The extracted surface defects 
were processed at different input parameters than what the entire bridge decks were processed at. In addition, each comparative ground truth measure was processed at similar input parameters allowing for a fair comparison.

\subsubsection{Ground Truth Measure for Comparison}

A technique developed by the Michigan Tech Research Institute, 3D Optical Bridgeevaluation System (3DOBS), was chosen to be the ground truth for comparison to the LiDAR technique. 3DOBS utilizes 3D photogrammetry to generate three-dimensional models from stereo pairs of electro-optical (EO) imagery. The developed models provide depth and height information that could not otherwise be determined from individual EO images. A digital single-lens reflex (DSLR), high-quality camera is the main component of the field system. The system implemented during the field demonstration consisted of a Nikon D5000 DSLR camera, vehicle mount and a camera triggering device as shown in Figure 4.11(de Melo e Silva and Brooks 2011).

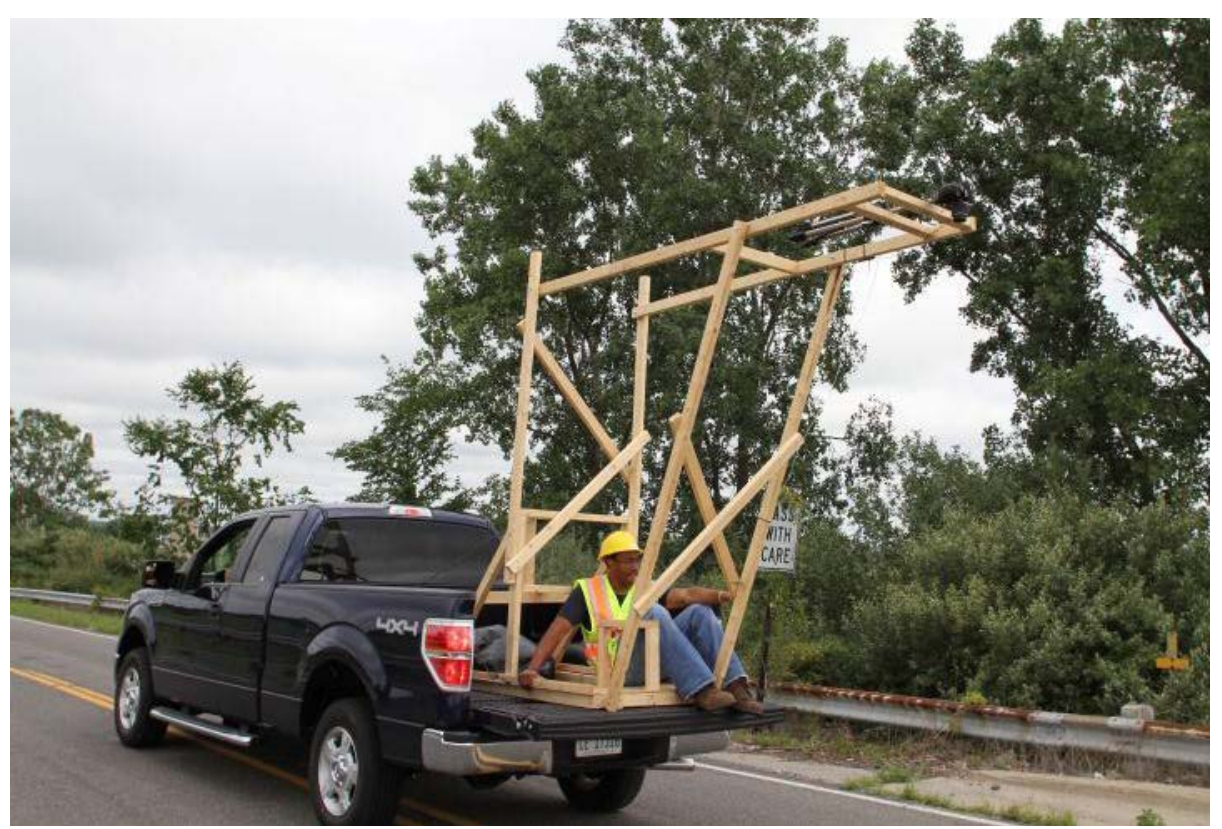

Figure 4.11: 3DOBS field demonstration data collection system (Courtesy of Renee Oats) (See Appendix D)

MTRI conducted laboratory testing on 3DOBS; the results showed that the technology is capable of detecting vertical changes smaller than 0.079 in $(2 \mathrm{~mm})$. With the current 
bridge inspection standard of 0.25 in $(6.35 \mathrm{~mm})$ for minimum consideration criteria of an elevation change to be a potential spall, the 3DOBS technology exceeds the established criteria (de Melo e Silva and Brooks 2011).

\subsection{2 “Poor” Bridge Selection}

For Mannsiding Road NB, the data collection method was slightly different than the other case studies as described before. With the two deck scans being located on opposite abutments and the northeast scan collect at medium resolution, the point cloud decay was evident closer to the northeast approach slab. The predicated spall area from the algorithm with the established input parameters was saturated with error at $56.2 \mathrm{ft}$ from the southwest approach to the northeast approach, as shown in Appendix C. As a result of the lowered resolution data capture on the northeast approach, only the southwest 56.2 feet of the Mannsiding Road NB data was applicable. The estimated percentage of defected surface area from the Leica ScanStation C10 data was 2.26\% of the relevant area. The established ground truth measure, 3DOBS, predicted that the defected surface area to be $1.08 \%$ of the same relevant area. Details of the findings are shown in Table 4.2 .

A spall of significant size was present within the established 56.2 foot capture radius of the southwest approach scan allowing for an evaluation of the capabilities of terrestrial LiDAR under ideal conditions. Figure 4.12 shows the location of the spall in reference to the entire deck, which is highlighted in red. The spall was evaluated with the algorithm under the established input spall parameters. Results from the analysis estimated that the surface area, volume and percent surface area were $2.065 \mathrm{ft}^{2}\left(0.285 \mathrm{~m}^{2}\right), 0.067 \mathrm{ft}^{3}(0.002$ $\mathrm{m}^{3}$ ) and $0.06 \%$ respectively. Figure 4.13 shows an optical image and the predicted spall area image from the algorithm for visual comparison. The green color in the predicted spall area image represents the assumed spalled area. 
Table 4.2: Results from the Mannsiding Road NB selected deck evaluation

\begin{tabular}{|c|c|c|c|}
\hline \multicolumn{3}{|c|}{ Results for Mannsiding Road NB } \\
\hline \multirow{2}{*}{$\begin{array}{c}\text { Items of } \\
\text { Comparison }\end{array}$} & Selected deck evaluation & \multirow{2}{*}{ Difference } \\
\cline { 2 - 3 } & LiDAR & 3DOBS & \\
\hline Bridge surface area & $1223.65 \mathrm{ft}^{2}\left(113.68 \mathrm{~m}^{2}\right)$ & - \\
\hline $\begin{array}{c}\text { Predicted defect } \\
\text { area }\end{array}$ & $\begin{array}{c}27.64 \mathrm{ft}^{2} \\
\left(2.57 \mathrm{~m}^{2}\right)\end{array}$ & $\begin{array}{c}13.18 \mathrm{ft}^{2} \\
\left(1.22 \mathrm{~m}^{2}\right)\end{array}$ & $\begin{array}{c}14.46 \mathrm{ft}^{2} \\
\left(1.34 \mathrm{~m}^{2}\right)\end{array}$ \\
\hline $\begin{array}{c}\text { Predicted defect } \\
\text { volume }\end{array}$ & $\begin{array}{c}2.33 \mathrm{ft}^{3} \\
\left(0.07 \mathrm{~m}^{3}\right)\end{array}$ & $\begin{array}{c}1.10 \mathrm{ft}^{3} \\
\left(0.03 \mathrm{~m}^{3}\right)\end{array}$ & $\begin{array}{c}1.23 \mathrm{ft}^{3} \\
\left(0.04 \mathrm{~m}^{3}\right)\end{array}$ \\
\hline $\begin{array}{c}\text { Predicted defect \% } \\
\text { area }\end{array}$ & $2.26 \%$ & $1.08 \%$ & $1.18 \%$ \\
\hline
\end{tabular}

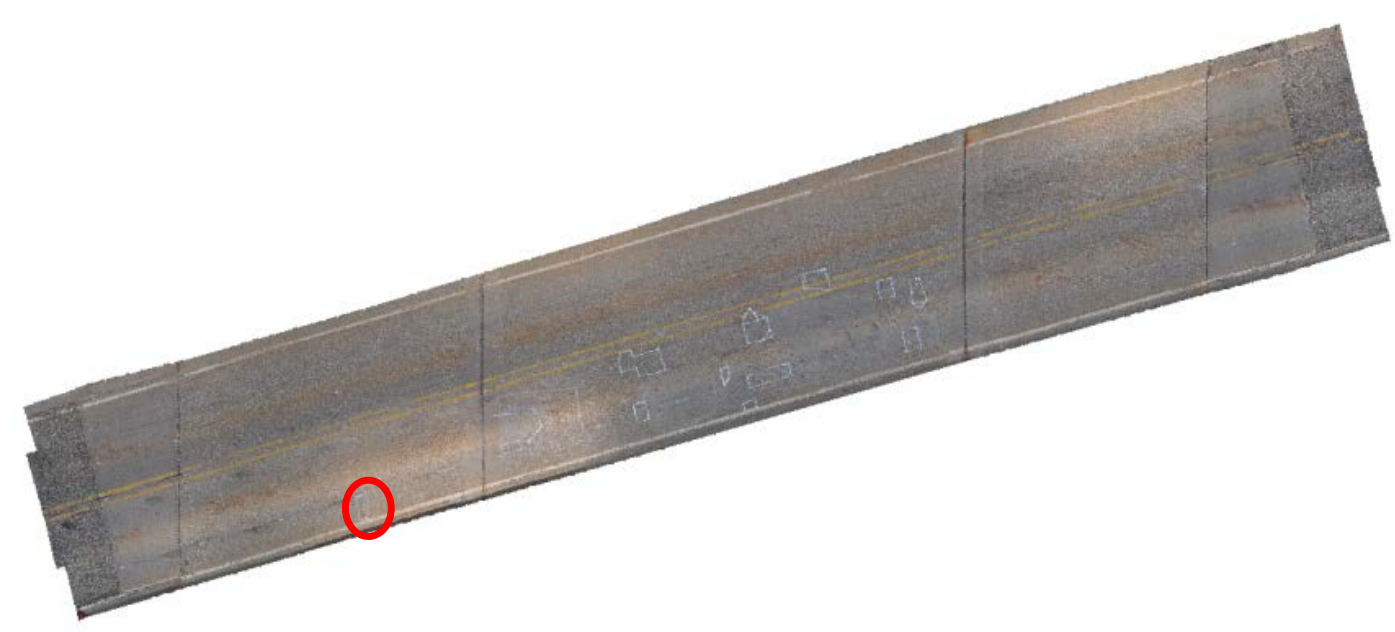

Figure 4.12: Optical image of Mannsiding Road NB showing evaluated spall location (Courtesy of MTRI) (See Appendix D) 

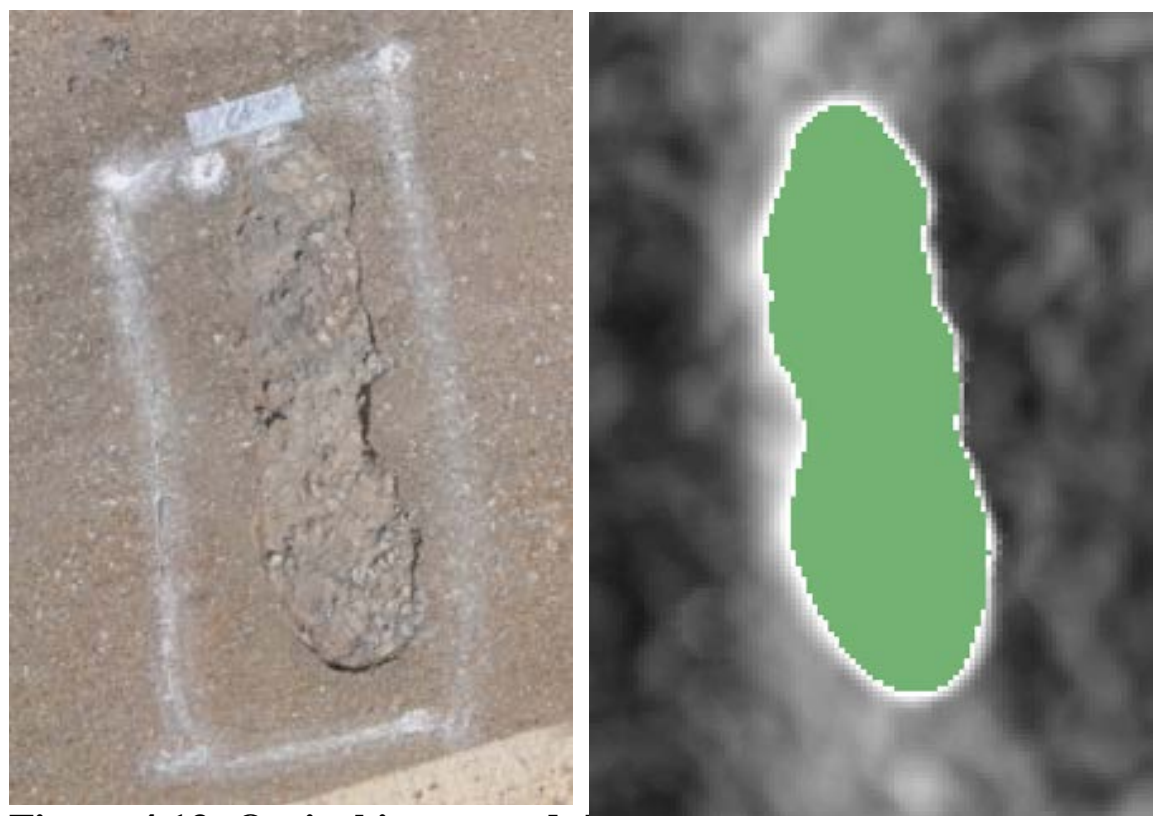

Figure 4.13: Optical image and the predicted spall area image for visual comparison (Courtesy of MTRI) (See Appendix D)

3DOBS estimated that the selected spall surface area, volume and percent surface were $1.696 \mathrm{ft}^{2}\left(0.158 \mathrm{~m}^{2}\right), 0.064 \mathrm{ft}^{3}\left(0.002 \mathrm{~m}^{3}\right)$ and $0.049 \%$, respectively. The differences in the three measures were $0.369 \mathrm{ft}^{2}\left(0.127 \mathrm{~m}^{2}\right), 0.003 \mathrm{ft}^{3}\left(0.000 \mathrm{~m}^{3}\right)$ and $0.011 \%$ demonstrating that terrestrial LiDAR possesses the capabilities of detecting surface defects to an acceptable degree of accuracy. Table 4.3 lists dimensions of the evaluated spall for LiDAR, 3DOBS and the differences. The Mannsiding Road NB demonstrated the sensitive nature of the data acquisition procedure and when a suspected defect is within the established maximum radius of capture, determination of the surface condition is possible. 
Table 4.3: Isolated section within capture radius on Mannsiding Road NB

\begin{tabular}{|c|c|c|c|}
\hline \multicolumn{4}{|c|}{ Isolated section within capture radius } \\
\hline $\begin{array}{c}\text { Items of } \\
\text { Comparison }\end{array}$ & LiDAR & 3DOBS & Difference \\
\hline $\begin{array}{c}\text { Predicted defect } \\
\text { area }\end{array}$ & $\begin{array}{c}2.065 \mathrm{ft}^{2} \\
\left(0.285 \mathrm{~m}^{2}\right)\end{array}$ & $\begin{array}{c}1.696 \mathrm{ft}^{2} \\
\left(0.158 \mathrm{~m}^{2}\right)\end{array}$ & $\begin{array}{c}0.369 \mathrm{ft}^{2} \\
\left(0.127 \mathrm{~m}^{2}\right)\end{array}$ \\
\hline $\begin{array}{c}\text { Predicted defect } \\
\text { volume }\end{array}$ & $\begin{array}{c}0.067 \mathrm{ft}^{3} \\
\left(0.002 \mathrm{~m}^{3}\right)\end{array}$ & $\begin{array}{c}0.064 \mathrm{ft}^{3} \\
\left(0.002 \mathrm{~m}^{3}\right)\end{array}$ & $\begin{array}{c}0.003 \mathrm{ft}^{3} \\
\left(0.000 \mathrm{~m}^{3}\right)\end{array}$ \\
\hline $\begin{array}{c}\text { Predicted defect \% } \\
\text { area }\end{array}$ & $0.06 \%$ & $0.049 \%$ & $0.011 \%$ \\
\hline
\end{tabular}

\subsection{3 “Fair” Bridge Selection}

For Willow Road, the two data collection methods pertaining to the evaluation of the bridge deck condition were high-resolution allowing for the densest point cloud generation possible. With the two deck scans being located on opposite abutments, the point cloud decay was evident along the bridge deck surface once the 56.2 foot maximum radius of capture was exceed from each approach slab. The predicted spall area from the algorithm with the established input parameters was saturated with error in the center portion of the deck point cloud, as shown in Appendix C. As a result only the data contained within the two established maximum radii was evaluated and the remaining information was deemed inadequate for analysis. The estimated percentage of defected surface area from the Leica ScanStation C10 data was 6.14\% of the selected bridge deck surface area. The established ground truth measure, 3DOBS, predicted that the defected surface area to be $3.73 \%$ of the selected bridge deck surface area. Details of the findings are shown in Table 4.4. 
Table 4.4: Results from the Willow Road selected deck evaluation

\begin{tabular}{|c|c|c|c|}
\hline \multicolumn{3}{|c|}{ Results for Willow Road } \\
\hline $\begin{array}{c}\text { Items of } \\
\text { Comparison }\end{array}$ & Selected deck evaluation & \multirow{2}{*}{ Difference } \\
\cline { 2 - 3 } & LiDAR & 3DOBS & \\
\hline Bridge surface area & $2712.17 \mathrm{ft}^{2}\left(251.97 \mathrm{~m}^{2}\right)$ & - \\
\hline $\begin{array}{c}\text { Predicted defect } \\
\text { area }\end{array}$ & $\begin{array}{c}166.46 \mathrm{ft}^{2} \\
\left(15.46 \mathrm{~m}^{2}\right)\end{array}$ & $\begin{array}{c}101.11 \mathrm{ft}^{2} \\
\left(9.39 \mathrm{~m}^{2}\right)\end{array}$ & $\begin{array}{c}65.35 \mathrm{ft}^{2} \\
\left(6.07 \mathrm{~m}^{2}\right)\end{array}$ \\
\hline $\begin{array}{c}\text { Predicted defect } \\
\text { volume }\end{array}$ & $\begin{array}{c}18.97 \mathrm{ft}^{3} \\
\left(0.54 \mathrm{~m}^{3}\right)\end{array}$ & $\begin{array}{c}21.23 \mathrm{ft}^{3} \\
\left(0.60 \mathrm{~m}^{3}\right)\end{array}$ & $\begin{array}{c}2.26 \mathrm{ft}^{3} \\
\left(0.06 \mathrm{~m}^{3}\right)\end{array}$ \\
\hline $\begin{array}{c}\text { Predicted defect } \% \\
\text { area }\end{array}$ & $6.14 \%$ & $3.73 \%$ & $2.41 \%$ \\
\hline
\end{tabular}

To further evaluate the capabilities of the technology in ideal conditions, a section of the north shoulder of the deck was selected, which was in a severely deteriorated state. The selected section of the north shoulder was within the 56.2 foot maximum radius of capture. Figure 4.14 shows the location of the selection section of the north shoulder in reference to the entire deck, which is highlighted in red. The shoulder was evaluated with the algorithm under the established input spall parameters. Results from the analysis estimated that the surface area, volume and percent surface area were $9.91 \mathrm{ft}^{2}\left(0.92 \mathrm{~m}^{2}\right)$, $0.65 \mathrm{ft}^{3}\left(0.02 \mathrm{~m}^{3}\right)$ and $0.18 \%$ respectively. Figure 4.15 shows an optical image and the predicted spall area image from the algorithm for visual comparison. The green color in the predicted spall area image represents the assumed spalled area. 


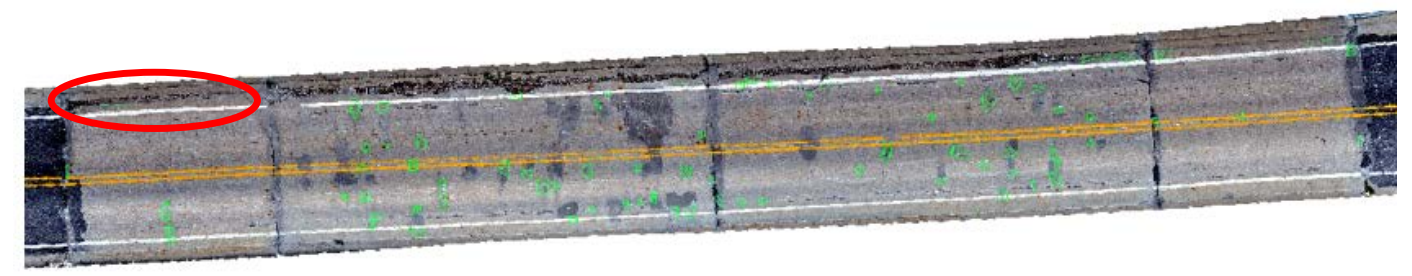

Figure 4.14: Optical image of Willow Road showing evaluated surface defect location (Courtesy of MTRI) (See Appendix D)
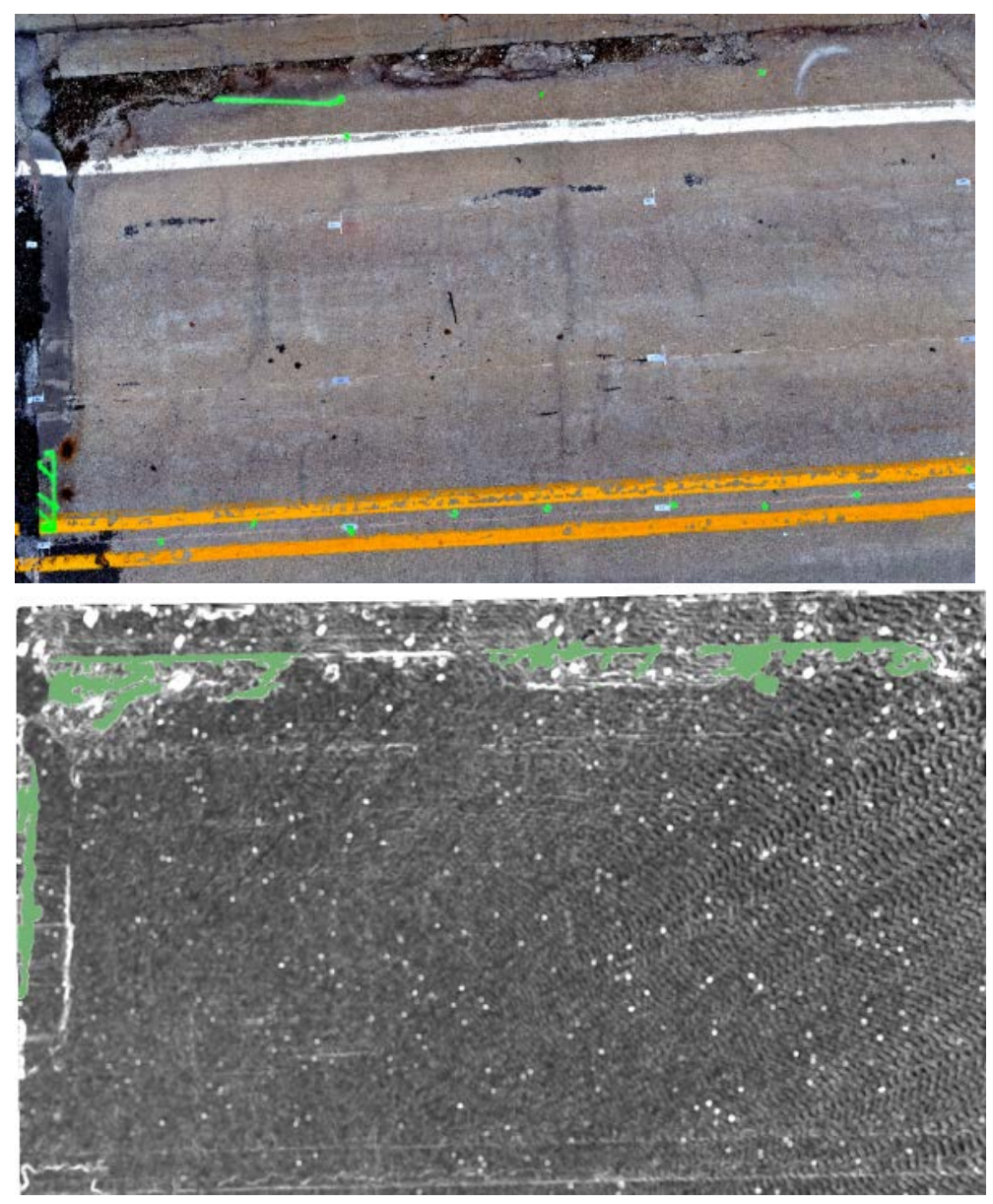

Figure 4.15: Optical image and the predicted spall area image for visual comparison 
3DOBS estimated that the selected spall surface area, volume and percent surface were $8.31 \mathrm{ft}^{2}\left(0.77 \mathrm{~m}^{2}\right), 0.60 \mathrm{ft}^{3}\left(0.02 \mathrm{~m}^{3}\right)$ and $0.15 \%$, respectively. The variations in the three measures were $1.60 \mathrm{ft}^{2}\left(0.15 \mathrm{~m}^{2}\right), 0.05 \mathrm{ft}^{3}\left(0.00 \mathrm{~m}^{3}\right)$ and $0.03 \%$, respectively. These results showed that when LiDAR is used within its limitations of maximum capture radius and resolution, accurate quantification of metrics can be made. Table 4.5 lists dimensions of the evaluated shoulder for LiDAR, 3DOBS and the differences.

Table 4.5: Isolated section within capture radius on Willow Road

\begin{tabular}{|c|c|c|c|}
\hline \multicolumn{4}{|c|}{ Isolated section within capture radius } \\
\hline $\begin{array}{c}\text { Items of } \\
\text { Comparison }\end{array}$ & LiDAR & 3DOBS & Difference \\
\hline $\begin{array}{c}\text { Predicted defect } \\
\text { area }\end{array}$ & $\begin{array}{c}9.91 \mathrm{ft}^{2} \\
\left(0.92 \mathrm{~m}^{2}\right)\end{array}$ & $\begin{array}{c}8.31 \mathrm{ft}^{2} \\
\left(0.77 \mathrm{~m}^{2}\right)\end{array}$ & $\begin{array}{c}1.60 \mathrm{ft}^{2} \\
\left(0.15 \mathrm{~m}^{2}\right)\end{array}$ \\
\hline $\begin{array}{c}\text { Predicted defect } \\
\text { volume }\end{array}$ & $\begin{array}{c}0.65 \mathrm{ft}^{3} \\
\left(0.02 \mathrm{~m}^{3}\right)\end{array}$ & $\begin{array}{c}0.60 \mathrm{ft}^{3} \\
\left(0.02 \mathrm{~m}^{3}\right)\end{array}$ & $\begin{array}{c}0.05 \mathrm{ft}^{3} \\
\left(0.00 \mathrm{~m}^{3}\right)\end{array}$ \\
\hline $\begin{array}{c}\text { Predicted defect \% } \\
\text { area }\end{array}$ & $0.18 \%$ & $0.15 \%$ & $0.03 \%$ \\
\hline
\end{tabular}

\subsection{4 “Satisfactory” Bridge Selection}

For Freer Road, the two data collection methods pertaining to the evaluation of the bridge deck condition were high-resolution allowing for the densest point cloud generation possible. With the two deck scans being located on opposite abutments, the point cloud decay was evident along the bridge deck surface once the 56.2 foot maximum radius of capture was exceed from each approach slab. The predicted spall area from the algorithm with the established input parameters was saturated with error in the center portion of the deck point cloud, as shown in Appendix C. As a result only the data contained within the two established maximum radii was evaluated and the remaining information was deemed inadequate for analysis. The estimated percentage of defected surface area from the Leica ScanStation C10 data was 1.68\% of the selected bridge deck surface area. The established ground truth measure, 3DOBS, predicted that the defected surface area to be 
$0.38 \%$ of the selected bridge deck surface area. Details of the findings are shown in Table 4.6.

Differing for Mannsiding Road NB and Willow Road field demonstrations, Freer Road contained no significant surface deterioration or shoulder distress. Evaluation under ideal conditions was unable to be completed on Freer Road due to the limited quantity of surface defects. The limited quantity of surface defects was expected; the selection parameters established during the field demonstration selection desired a case study bridge with a rating of "satisfactory" under the NBI rating system.

Table 4.6: Results from the Freer Road selected deck evaluation

\begin{tabular}{|c|c|c|c|}
\hline \multicolumn{3}{|c|}{ Results for Freer Road } \\
\hline \multirow{2}{*}{$\begin{array}{c}\text { Items of } \\
\text { Comparison }\end{array}$} & \multicolumn{2}{|c|}{ Selected deck evaluation } & \multirow{2}{*}{ Difference } \\
\cline { 2 - 3 } & LiDAR & 3DOBS & \\
\hline Bridge surface area & $2970.24 \mathrm{ft}^{2}\left(275.94 \mathrm{~m}^{2}\right)$ & - \\
\hline $\begin{array}{c}\text { Predicted defect } \\
\text { area }\end{array}$ & $\begin{array}{c}49.82 \mathrm{ft}^{2} \\
\left(4.63 \mathrm{~m}^{2}\right)\end{array}$ & $\begin{array}{c}11.32 \mathrm{ft}^{2} \\
\left(1.05 \mathrm{~m}^{2}\right)\end{array}$ & $\begin{array}{c}38.50 \mathrm{ft}^{2} \\
\left(3.58 \mathrm{~m}^{2}\right)\end{array}$ \\
\hline $\begin{array}{c}\text { Predicted defect } \\
\text { volume }\end{array}$ & $\begin{array}{c}8.52 \mathrm{ft}^{3} \\
\left(0.24 \mathrm{~m}^{3}\right)\end{array}$ & $\begin{array}{c}0.34 \mathrm{ft}^{3} \\
\left(0.01 \mathrm{~m}^{3}\right)\end{array}$ & $\begin{array}{c}8.18 \mathrm{ft}^{3} \\
\left(0.23 \mathrm{~m}^{3}\right)\end{array}$ \\
\hline $\begin{array}{c}\text { Predicted defect \% } \\
\text { area }\end{array}$ & $1.68 \%$ & $0.38 \%$ & $1.30 \%$ \\
\hline
\end{tabular}

\subsection{5 “Supplemental” Bridge Selection}

For Mannsiding Road SB, the short span of the bridge and the collection of data from the two established locations at high-resolution produced the most dense point cloud over the entire bridge deck. Limited point cloud decay was visible in the spall algorithm output, as shown in Appendix C. The estimated percentage of defected surface area from the Leica ScanStation C10 data was 3.1\% of the total bridge deck surface area. The established ground truth measure, 3DOBS, predicted that the defected surface area to be $1.4 \%$ of the total bridge deck surface area. Details of the findings are shown in Table 4.7. 
With the short span and high-resolution data collection techniques performed at the two established scan locations, the estimated defected metrics were similar. Also to be noted is that the profile graph provided in the previous section showed that the bridge had negligible arch and was sloped from the southwest to the northeast approach. The absence of the arch and the sloped surface allowed for an increase in the coverage angle, further allowing for increased line of slight from the receiver to the middle span of the bridge.

Table 4.7: Results from the Mannsiding Road SB full deck evaluation

\begin{tabular}{|c|c|c|c|}
\hline \multicolumn{3}{|c|}{ Results for Mannsiding Road SB } \\
\hline \multirow{2}{*}{$\begin{array}{c}\text { Items of } \\
\text { Comparison }\end{array}$} & \multicolumn{2}{|c|}{ Total deck evaluation } & \multirow{2}{*}{ Difference } \\
\cline { 2 - 3 } & LiDAR & 3DOBS & \\
\hline Bridge surface area & $3403.2 \mathrm{ft}^{2}\left(316.5 \mathrm{~m}^{2}\right)$ & - \\
\hline $\begin{array}{c}\text { Predicted defect } \\
\text { area }\end{array}$ & $\begin{array}{c}106.40 \mathrm{ft}^{2} \\
\left(9.90 \mathrm{~m}^{2}\right)\end{array}$ & $\begin{array}{c}46.50 \mathrm{ft}^{2} \\
\left(4.32 \mathrm{~m}^{2}\right)\end{array}$ & $\begin{array}{c}59.90 \mathrm{ft}^{2} \\
\left(5.58 \mathrm{~m}^{2}\right)\end{array}$ \\
\hline $\begin{array}{c}\text { Predicted defect } \\
\text { volume }\end{array}$ & $\begin{array}{c}6.60 \mathrm{ft}^{3} \\
\left(0.18 \mathrm{~m}^{3}\right)\end{array}$ & $\begin{array}{c}2.94 \mathrm{ft}^{3} \\
\left(0.08 \mathrm{~m}^{3}\right)\end{array}$ & $\begin{array}{c}3.66 \mathrm{ft}^{3} \\
\left(0.10 \mathrm{~m}^{3}\right)\end{array}$ \\
\hline $\begin{array}{c}\text { Predicted defect \% } \\
\text { area }\end{array}$ & $3.1 \%$ & $1.4 \%$ & $1.7 \%$ \\
\hline
\end{tabular}

The evaluation under ideal conditions was unable to be completed on Mannsiding Road SB due to the limited quantity of surface defects. The limited quantity of surface defects was unexpected, because the "Supplemental” bridge was not initially investigated and surface defects were not documented. However, the results showed that the potential for the arch and angle of the bridge could greatly affect the clarity of collected data. 


\section{Summary}

\subsection{Sensing Bridge Surface Condition}

The research conducted concluded that the point cloud decay rate is not linear, but rather exponential. When the data was collected at a rate of 50,000 points/second, the exponential decay resulted in the establishment of a maximum coverage radius of $56.2 \mathrm{ft}$. Evaluation of the entire bridge deck data was performed to reinforce the conclusion of the exponential point cloud decay and the maximum coverage radius. Full bridge deck results when compared to the established ground truth of 3DOBS demonstrated that the limited point cloud density in the middle of the field demonstration data developed an overestimation of the damage surface. The difference in the calculated percentage of damage surface area for the "Poor", "Fair" and "Satisfactory" between the LiDAR and 3DOBS data was $55.93 \%, 34.6 \%$, and $14.56 \%$ respectively. No definitive trend can be established, because of the limited amount of unique field demonstration locations.

However, the two damaged features located within the maximum radius of coverage on Mannsiding Road NB and Willow Road showed the potential of the technology. The difference in the calculated percentage of the damaged features located on Mannsiding Road NB and Willow Road between the LiDAR and 3DOBS data were $0.011 \%$ and $0.03 \%$, respectively. The small variation between the two technologies definitively shows that terrestrial LiDAR has the capabilities of sensing surface defects to an acceptable accuracy.

\subsection{Pros and Cons of the Modeling Software}

The two three-dimensional modeling programs utilized for filtering and post-processing the collected LiDAR data had both associated positives and negatives. Reevaluating the developed criteria for software selection of user-friendliness, cost, accuracy, what the modeling program was designed for and current federal and state agency uses, several conclusions were generated. With regards to the MTRI 3DOBS spall detection algorithm, 
user critiques were provide for further development of the user interface and code parameters.

\subsubsection{Certainty 3D’s TopoDOT Modeling Software}

The Certainty 3D’s TopoDOT modeling software provided a user-friendly interface with available options to effectively filter LiDAR collected data. With the TopoDOT software utilizing the Bentley MicroStation platform and function configuration, previous MicroStation experience helped accelerate the learning process (Bentley Systems 2011). The necessary LiDAR functions are located in a single toolbar, which allowed the user to perform the necessary operations for filtering the LiDAR data with limited distraction by non-essential functions not pertaining to the filtering process. Additionally, the free web based one-on-one instruction enhanced the software experience and allowed for clarification on the capabilities of the LiDAR specific functions.

For the current LiDAR applications present in the civil engineering industry, the software allowed for easy navigation and feature selection/extraction in a complex threedimensional model. The software was originally designed for terrestrial and mobile LiDAR data pertaining to civil infrastructure projects, which required minor manipulation of the developed LiDAR functions. When considering the unutilized potential of LiDAR technology and the limited processing software available, the Certainty 3D's TopoDOT modeling software is among the most complete software packages. However, due to the limited LiDAR processing software providers, the cost for a single license of TopoDOT is fairly high and the requirement of the Bentley MicroStation software to access TopoDOT increases the associated cost.

Recommendations for improving the Certainty 3D's TopoDOT modeling software for the use in the bridge inspection realm would be centered on the high capital cost associated with the software and the additional software purchases. Pertaining to the data filtering and processing, the ability to rapidly extracted data of CoRe structural elements would increase the effectiveness of the software. With the CoRe structural element data 
extracted, the user could develop a meshed surface within MicroStation. The meshed surface could be used to visually display surface defects and generate volume calculation of damaged material. With the experience gathered from the performed research, the Certainty 3D's TopoDOT modeling software can be classified as a powerful LiDAR filtering and processing tool with limited missing components.

\subsubsection{Quick Terrain Modeler}

With the additional LiDAR data filtering required to properly evaluate the effectiveness of terrestrial LIDAR as an inspection tool, Quick Terrain Modeler was utilized. Limited exploration into the software's capabilities were conducted due to the finite tasks remaining to be performed on the TopoDOT filtered LiDAR data. However, some concerns were raised regarding the software's selection criteria after software use. The Quick Terrain Modeler provided a simply user-friendly interface, which allowed for limited confusion during the data refinement. The provided LiDAR functions were elegant, which required little instruction or further investigation into the proper procedure for executing a command.

When the required filtering tasks were performed with Quick Terrain Modeler, the user noticed subtle intricacies, which alluded to the original intent of the software as aerial LiDAR processing software. These intricacies required the user to manipulate the original functionality of the software and repurpose the provided tools for terrestrial LiDAR data, requiring additional processing time and assumptions. The main drawback from the Quick Terrain Modeler software was the three-dimensional navigation, which allowed for limited user control. With the inputted data already filtered down to the pertinent bridge deck data, the navigation issue was negligible due to the twodimensional nature of the deck data. When the deck data was broken into the established subsets, necessary for the development of the geotiff DEMs, the mensuration tool was used to confirm the subset lengths. The mensuration tool was rather cumbersome, with no feature snap functionality, the selection of the pertinent information and desired location was questionable. Software improvements may be necessary to gain acceptance 
in the civil engineering industry and perform efficient data processing on terrestrial or mobile LiDAR data sets for bridge deck applications.

Recommendations for improving the Quick Terrain Modeler software for the use in the bridge inspection realm would be centered on the navigation capabilities around a complex three-dimensional element and the mensuration tool. By incorporating multiple viewing windows into the user interface, the software would become more versatile in navigating around complex terrestrial and mobile LiDAR data. The redesign of the mensuration tool is necessary for acceptance into the civil engineering realm. The addition on a snap feature would allow for the user to confidently measure pertinent element metrics. With the experience gathered from the performed research, the Quick Terrain Modeler software can be classified as a useful LiDAR filtering and processing tool, but requires further development.

\subsubsection{MTRI 3DOBS Spall Detection Algorithm}

The MTRI 3DOBS Spall Detection Algorithm allowed for automated spall detection with limited user input required. The MTRI developed script used to perform the automated spall detection had a relatively simple interface, but the potential of the system was evident. With only six required user defined parameters, three of which were locating the necessary input files and output directory, the limited user interaction allowed for limited generation of error during this phase. The commentary provided during the execution of the script allowed for the user to follow the methodology and locate issues if the script crushed. The MTRI 3DOBS Spall Detection Algorithm provided a simple user interface, which utilized ESRI ArcMap geo-spatial analysis tools to evaluate inputted data efficiently.

When considering the practicality of the required evaluation parameters for classifying a spall issues arose. For the current bridge inspection standards for spall classification to be inputted into the algorithm, knowledge of the evaluated geotiff DEM was necessary and conversion from inches or centimeters to pixels and standard deviation was required. 
The determination of these input parameters resulted in an iterative process, which generated delays and assumptions. Additionally, when no metrics were generated to populate a particular ESRI ArcMap layer at the set input parameters, the script would fail. The result was the requirement to define the input parameters such that a metric was generated for each described layer within the algorithm, which resulted in an overestimation of the spalled area. Script improvements are necessary to enhance the viability of the algorithm and gain acceptance in the civil engineering industry with regards to terrestrial LiDAR data.

Recommendations for improving the MTRI 3DOBS Spall Detection Algorithm for the use in the bridge inspection realm would be centered on the input spall classification parameters necessary to execute the proper running of the spall detection script. By fixing the spall input parameters to current bridge inspection standards, the iterative process would be removed, resulting in efficient data processing and usable bridge surface metrics. The ability to produce sub-millimeter measurements are impractical when considering the current inspection standards and the ability to effectively present spall measures pertaining to current standards would be preferable. With the experience gathered from the performed research, the MTRI 3DOBS Spall Detection Algorithm can be classified as a useful surface bridge evaluation tool, but requires further development.

\subsection{Implementation of LiDAR for Bridge Inspections}

The implementation and repurposing of terrestrial LiDAR will be important for applying this technology in the bridge inspection field. With the current use of terrestrial LiDAR in the civil engineering industry as an as-built surveying tool and an inventory data generator, limited repurposing of the collection process would be required. The technology would be applicable to any concrete surface inspection, allowing for accurate generation of surface condition to a certain degree. The utilized data collection method conducted during the full-scale field demonstration resulted in the need for bridge closure and inspector exposure to traffic. To eliminate the bridge closure need and remove the inspector from a potentially harmful situation, a mobile LiDAR platform could be 
utilized. The mobile system is typically mounted on a vehicle, which can move across the bridge at a constant rate gathering information along the entire bridge deck. Additional testing and evaluation of LiDAR are required to develop a remote sensing system that is capable of collecting viable bridge surface metrics at near highway speeds.

By having the bridge inspector collect the data with a terrestrial LiDAR platform, the inspector would be able to ensure the proper areas are sensed and modeled to generate an accurate surface assessment. The bridge inspector would be allowed to perform a routine visual inspection, while gathering the necessary LiDAR information and classifying areas of concern requiring additional evaluation. However, the issue of traffic disruption and inspector exposure remains a concern.

By utilizing a mobile LiDAR system, the inspector could collect the necessary information from the safety of a vehicle. The drawback to mobile LiDAR collection is the ability to visually discern between debris and potential surface defect. The visual inspection requirement could be accomplished by incorporating an optical camera mounted on the vehicle with the capabilities of capturing still images with limited blur due to the vehicle speed. The captured optical images could then be fused together generating a master bridge deck image, which would allow for defect determination. Mobile LiDAR would alleviate traffic disruption and reduce inspector exposure, but further investigation and evaluation is necessary.

Recommendations for implementing a terrestrial LiDAR system for bridge surface evaluation would require significant traffic disruption and multiple scan locations would be necessary to develop a dense point cloud. The closer the scan locations are to one another the better the ability to measure deterioration mechanisms will be. With the terrestrial LiDAR unit used in this research, the maximum allowable distance between each scan location at a collection rate of 50,000 points/second would be $112.4 \mathrm{ft}$ or two times the maximum coverage radius of $56.2 \mathrm{ft}$. As discussed in the previous section, the point cloud decays exponentially as the distance away from the receiver increases. When incorporating the point cloud decay and laser dispersion rates, the limiting factor for a 
terrestrial LiDAR system is distance. The current state of terrestrial LiDAR and the findings from this research show that the implementation of a remote sensing system utilizing terrestrial LiDAR would not be practical for enhancing the bridge inspection practice without significant traffic disruption. Terrestrial LiDAR has the potential to assess other defect locations, such as areas on the underside and fascia, which would be difficult for mobile LiDAR or 3D photogrammetric systems. However, the findings show that the theory works and with further research into mobile LiDAR, the potential for a remote sensing, highway speed surface defect detection system could be feasible. 


\section{Conclusions and Future Work}

The goal of this thesis was to evaluate the surface defect detection capability of terrestrial LiDAR for reinforced concrete bridge decks. The evaluation of the terrestrial LiDAR system was accomplished through the collection and processing of data from four separate field demonstration locations. Processing allowed for characterization of the minimum resolvable surface defect, maximum conservable distance from LiDAR unit for detection, determination of point cloud density decay and algorithm limitations. While these characterizations allow for current federal, state and private agencies to repurpose current terrestrial LiDAR systems to enhance the reinforced concrete bridge deck inspection practice, there are still many improvements which can be made.

\subsection{Conclusions}

From the research conducted herein, the following conclusions were reached:

- The evaluated Leica ScanStation C10 terrestrial LiDAR unit can sense surface defects to a certain degree of accuracy, but is highly-dependent on five parameters; angle of incidence, coverage angle, collection rate, reflectivity, height of collection platform.

- The coverage angle was shown to rapidly decrease the further away from the terrestrial LiDAR unit. The angle of incidence increased at a similar magnitude to the coverage angle. The result was limited return of scattered light the further away from the receiver the object of interest became.

- Point cloud density does not decay in a linear fashion, but rather as an exponential function that approaches an asymptote between the centers of the two scan locations. The result of the exponential point cloud decay was the establishment of a maximum radius of capture of $56.2 \mathrm{ft}$ for the Leica ScanStation C10, collecting at 50,000 point/second. 
- Surface damage sensed within the maximum coverage radius was comparable to the established ground truth measure, 3DOBS, with relative accuracy. The difference in the calculated percentage of the sample damaged features located on Mannsiding Road NB and Willow Road between the LiDAR and 3DOBS data were $0.011 \%$ and $0.03 \%$, respectively. The small variation between the two technologies shows that terrestrial LiDAR has the capabilities of sensing surface defects to an acceptable accuracy.

\subsection{Future Work}

Areas have been identified where future studies are warranted. These areas were outside the scope of the current research and would require additional study and/or testing to fully investigate. Suggestions for future work include the following:

- Current literature regarding performance evaluation research for terrestrial LiDAR systems has mainly performed spherical target recognition precision testing. Spherical target always presents a constant detectable surface area, which allows for limited angle of incidence to be developed. By evaluating a planar surface at varying distances, a firm understanding of the target recognition precision would be established to better modeling bridge surface defects.

- With the issue of the dramatic point cloud density fall off as the object distance from the terrestrial LiDAR system increased, it was theorized that the reduction in the coverage angle associated with the bridge induced chamber or arch. A study should be conducted on the relative effect of volume estimation by introducing camber into a test specimen with a constant artificial defect present.

- The investigation into alternative LiDAR platforms, such as aerial and mobile for highway infrastructure inspection purposes should be conducted. By transitioning to a moving platform LiDAR system, uniform point cloud density maybe 
obtainable. A literature review should be conducted pertaining to the state of the practice to gain a complete understanding of the moving LiDAR platforms. Laboratory and field testing could follow, if deemed feasible.

- Determination of the reinforced concrete bridge deck's CoRe structural element condition state would further enhance the measurable performance metric. Additional refinement of the data collection method would be necessary and evaluation of the reinforced concrete deck underside would be required to establish a proper condition state according to AASHTO.

- Research seems warranted into the capabilities of multi-platform LiDAR systems for evaluation and surface defect detection of aged asphalt. With aged asphalt, the light reflectance index is raised due to the loss of asphalt binder, which may allow for substantial contrast development. A study should consider the elevated light reflectance between aged and fresh asphalt. Detectable surface defects should be evaluated, if found feasible. 


\section{References}

AASHTO. 2011. AASHTO Guide Manual for Bridge Element Inspection. 1 ed.: American Association of State Highway and Transportation Officials. p. 3-3.

Ahlborn TM, Shuchman R, Sutter LL, Brooks CN, Harris DK, Burns JW, Endsley KA, Evans DC, Vaghefi K, Oats RC. 2010. An Evaluation of Commercially Available Remote Sensors for Assessing Highway Bridge Condition. Michigan Technological University.

ASTM. 2008. Standard practice for computing international roughness index of roads from longitudinal profile measurements. ASTM E1926-08. American Society for Testing and Materials.

Bentley Systems I. 2011. Micro Station V8i. Bentley Systems, Inc.

Certainty D. 2011. TopoDOT. Orlando, FL: Certainty, 3D.

LiDAR Hardware [Internet]. 2011. [Updated 2011 Dec 10, cited 2012 Mar 29]. Available from:

www.cflha.gov/programs/techdevelopment/geotech/LiDAR/documents/04_chapter_2_Li $\underline{\mathrm{DAR}}$

Chu X. 2011. Fundamentals of LiDAR Remote Sensing. CU-Boulder. p. 1-27.

de Melo e Silva H, Brooks C. 2011. Summary of Field Demonstration Including Sensor Evaluation and Update of the DSS. Tech memo 21. Houghton, MI: Michigan Technological University. p. 58. 
Falkner E. 1995. Aerial Mapping: Methods and Applications. Boca Raton, FL: Lewis Publishers - CRC Press p. 322

Deficient Bridges by State and Highway System [Internet]. 2010. United States Department of Transportation. [Updated 2012 Jan 7, cited 2011 Dec 15]. Available from: http://www.fhwa.dot.gov/bridge/deficient.cfm

FHWA. 2004. National Bridge Inspection Standards. Federal Register. Federal Highway Administration Department of Transportation. p. 74419-74439.

FHWA. 2006. Bridge Inspector's Reference Manual (BIRM). Washington, D.C.

Fuchs PA, Washer GA, Chase SB, Moore M. 2004. Applications of Laser-Based Instrumentation for Highway Bridges. Journal of Bridge Engineering:9.

Gastineau A, Johnson T, Schultz A. 2009. Health Monitoring and Inspection - A Survey of Methods. Department of Civil Engineering University of Minnesota.

Geosystems L. 2011. ScanStation C10.

Gillespie TD. 1992. Everything You Always Wanted to Know about the IRI, But Were Afraid to Ask! Ann Arbor, MI: The University of Michigan Transportation Research Institute.

Hiremagalur J, Yen KS, K. A, T. B, Lasky TA, Ravani B. 2007. Creating Standards and Specifications for the Use of Laser Scanning in Caltrans Projects. Davis, CA: University of California, Davis. 
Imagery A. 2011. Quick Terrain Modeler (USA). In: x64, editor. Silver Spring, MD: Applied Imagery.

Laefer DF, Gannon J, Deely E. Reliability of Crack Detection Methods for Baseline Condition Assessments. Infrastructure systems [Internet]. [cited 2012 February 20, 2012];16:9.

MDOT. 2011a. Bridge deck preservation matrix- decks with epoxy coated rebar (ECR). Lansing, MI: Michigan Department of Transportation

MDOT. 2011b. Transportation Management System (TMS). In: Transporation MDo, editor.: Michigan Department of Transporation

MTRI. 2011. MTRI 3DOBS Spall Detection Algorithm Ann Arbor, MI: Michigan Tech Research Institute.

NCHRP. 2007. Bridge Inspection Practices - NCHRP Synthesis 375. Washington DC: Transportation Research Board.

Nowak AS, Szerszen MM, Juntunen DA. Michigan deck evaluation guide.

Reay S, Appleyard M, Van Dam T, Sandberg LB. 1998. Sealing and Filling of Cracks for Bituminous Concrete Pavements. Michigan Technological University.

Riegl U. 2011. LMS-Z210ii.

Schnitzler H-u, Moss CF. Echolocation.181-182. Available from:

http://www.bsos.umd.edu/psyc/batlab/publication/Echolocation.pdf 
Scott M, Rezaizadeh A, Delahaza A, Santos CG, Moore M, Graybeal B, Washer G. A comparison of nondestructive evaluation methods for bridge deck assessment 245-255.

Wanqiu L, Shen-En C. Reliability Analysis of 3D LiDAR Bridge Evaluation.

Nondestructive Characterization for Composite Material, Aerospace Engineering, Civil Infrastructure, and Homeland Security [Internet]. [cited 2012 February 20, 2012];7983:13. 


\section{Appendix A: MDOT Field Demonstration Inspection Reports}

Michigan Department of Transportation Form P2502 (02/2002)

Bridge Safety Inspection Report

Page 1 of 2

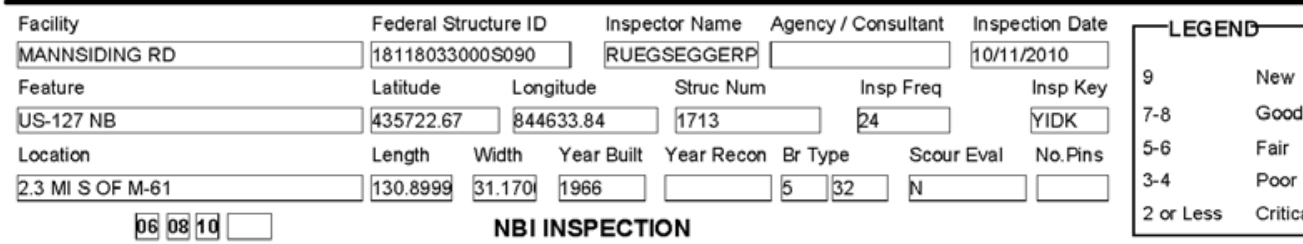

Deck

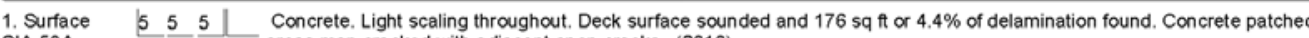

SIA-58A areas map cracked with adjacent open cracks. (2010), Concrete. Deck surface sounded and $176 \mathrm{sq} f \mathrm{ft} 4.4 \%$ of delamination found. Concrete patched areas map cracked with adjacent open cracks. (2008)

Conc. between $2 \%-10 \%$ of deck crked with conc. patched areas. $06(2006)$

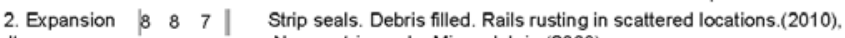

Jts $\quad$ Newer strip seals. Minor debris.(2008),

3. Other $\quad 6 \quad 5 \quad 4 \quad$ HPR at end joints. Adhesion failure along both, $100 \%$ at west. Chips in adjacent concrete. Minor evidence of leakage

Joints $\quad$ observed from underneath west joint, est. $5 \% .(2010)$, HPR at end jts. Minor chips and adhesion failure.(2008). HPR at end jts. Minor chips and adhesion failure. 06(2006).

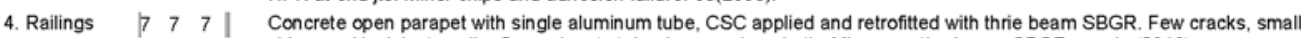
chips, and incipient spalls. Several rust stained areas along both. Minor weathering on SBGR panels.(2010), Conc parapet with 1 tube aluminum with thrie BM. retro fit. Vert \& Long. crks. CSS applied.(2008), Conc parapet with 1 tube aluminum with thrie BM. retro fit. Vert \& Long. crks. CSS applied. 06(2006).

5. Sidewalks $\mathrm{N} \mathrm{N} \mathrm{N}$

or curbs

6. Deck $\left|\begin{array}{llll}4 & 4 & 4\end{array}\right|$

SIA-58

Deck surface sounded and $176 \mathrm{sq} f \mathrm{ft}$ or $4.4 \%$ of delamination found. Concrete patches are map cracked. Deck underside sounded during detailed soffit inspection, $623 \mathrm{sq} f \mathrm{for} 15 \%$ distress. False decked by crews over traffic. Deterioration of the combined area of the top and bottom surface of the deck 10\%.(2010),

Deck surface sounded and $176 \mathrm{sq} f \mathrm{ft}$ or $4.4 \%$ of delamination found. Concrete patched areas map cracked. Span $2 \mathrm{~W}$ has several large areas of wet leaching map crked areas in sofit. Many trans. and diag. crks. on surface. Many long. and trans. crks. in soffit most of them leaching. Deck underside sounded during detailed soffit inspection, $623 \mathrm{sq} f \mathrm{for} 15 \%$ distress. False decked by crews over traffic. Deterioration of the combined area of the top and bottom surface of the deck $10 \% .(2008)$

Span $2 \mathrm{~W}$ has several large areas of wet leaching map crked areas in sofit. Many trans. and diag. crks. on surface. Many long. and trans. crks. in sofit most of them leaching. apprx. 200ft sq spalled or delam. on surface. Newer concrete patches on surface. Diag. leaching corner crks. at the NE. See general notes. 06(2006).

7. Drainage | Minor debris along brush blocks.(2010)

No problems noted. (2008)

No problems noted. 06(2006).

8. Stringer $\quad 6 \quad 6 \quad 6 \quad \mathrm{PCl}$ beams. CSC applied to fascias and beam ends at piers. Several minor scrapes and chips on beam $6 \mathrm{~S}$ over right lane

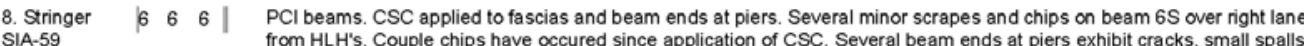
from HLH's. Couple chips have $\propto$ ccured since application of

and/or incipeint spalls, mainly adjacent to sole plates.(2010), PCl beams. Minor chips from HLH on BM 6 S. no steel exp Minor chips from HLH on BM 6 S. no steel exposed. Several Bm ends over Prs W/ight Crks \& Incip spalls. CSS applied to bm. ends at piers and fascias. 06(2006).

9. Paint $\quad \mathrm{N} N \mathrm{~N}-$

10. Section $\mathrm{N} N \mathrm{~N}$

Loss

11. Bearings $6 \begin{array}{lllll}6 & 4 & 4 & 4\end{array}$

Elasomeric bearing pads. Steel plates have moderate corrosion. Some minor Crks \& deformations on Elast Brgs over Prs 06 . Bolster at P2w. delaminated / fractured under beam 3s. in span 3w. causing loss of bearing area. Rating lowered due to loss of bearing support. Noted area marked with paint.(2010),

Elasomeric bearing pads. Steel plates have moderate corrosion. Some minor Crks \& deformations on Elast Brgs over Prs. 06 . Bolster at P2w. delaminated / fractured under beam $3 \mathrm{~s}$. in span $3 \mathrm{w}$. causing loss of bearing area. Rating lowered due to loss of bearing support. Noted area marked with paint.(2008),

Neopreme pads. Steel plates have moderate corrosion. Some minor Crks \& deformations on Elast Brgs over Prs. 06(2006)

$$
\text { Substructure }
$$

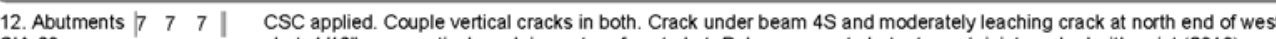
SIA-60 abut. 1/16" open vertical crack in center of east abut. Delam. on east abut. at const. joint marked with paint.(2010). Both have vertical cracks with some leaching, also an incipent spall at const. joint. E. abut. CSS applied.(2008), Both have vertical cracks with some leaching, also an incipent spall at const. joint. E. abut. CSS applied. 06(2006)

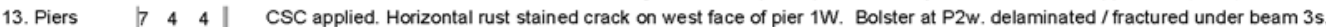

SIA-60 $\quad \begin{array}{llllll}7 & 4 & 4\end{array} \mid$ in span $3 w$. causing approx. $50 \%$ loss of bearing capacity. Rating lowered due to condition of bolster.(2010). in span $3 w$. causing approx. $50 \%$ loss of bearing capacity. Rating lowered due to condition of bolster. (2010), Piers have conc. repairs and CSS applied. Bolster at P $2 w$. delaminated / fractured under Piers have conc, repairs and CSS applied. 06(2006).

Figure A.1: Mannsiding Road NB routine bridge inspection report (MDOT 2011b) 


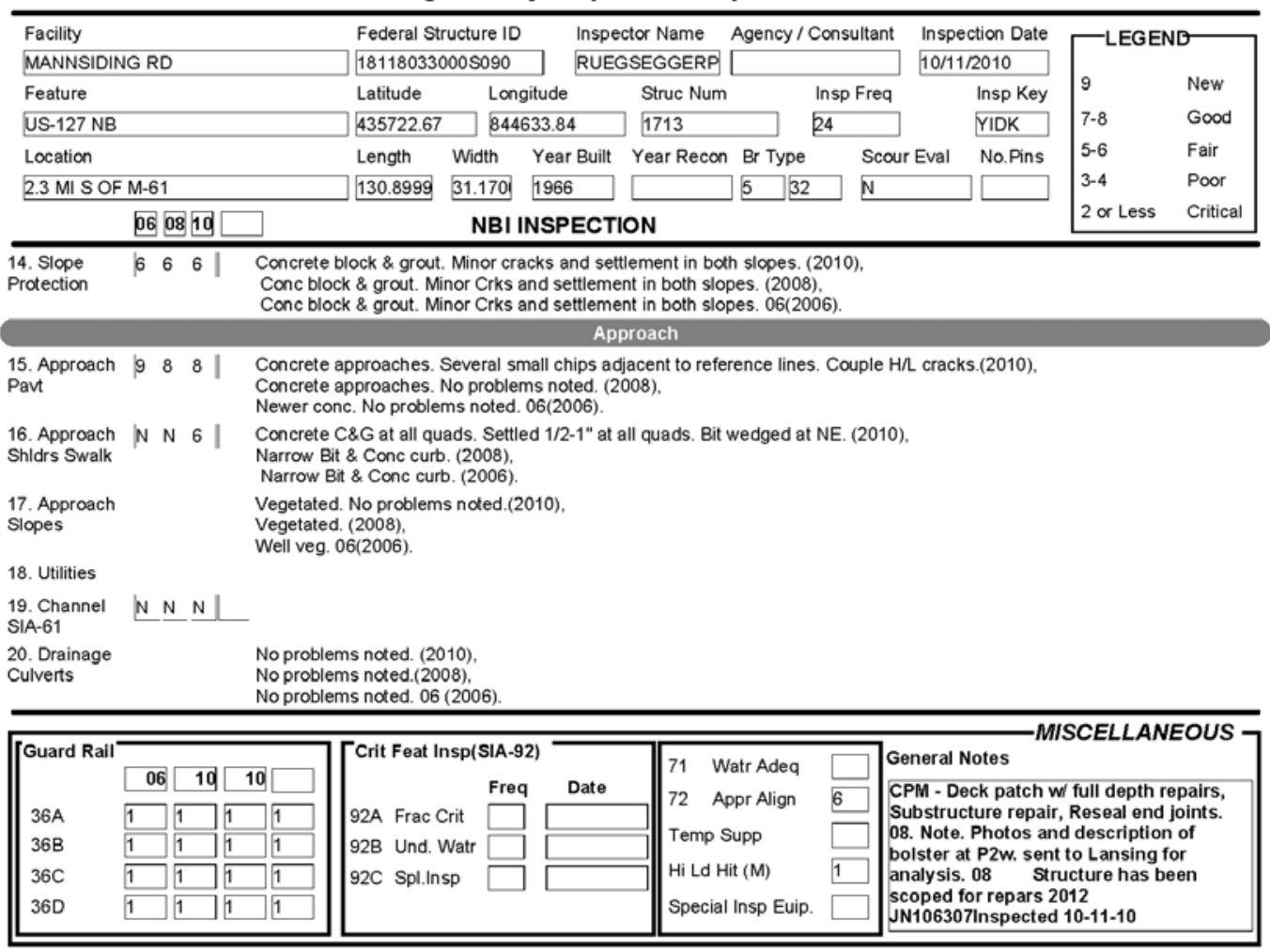

Figure A.1, continued. (MDOT 2011b) 


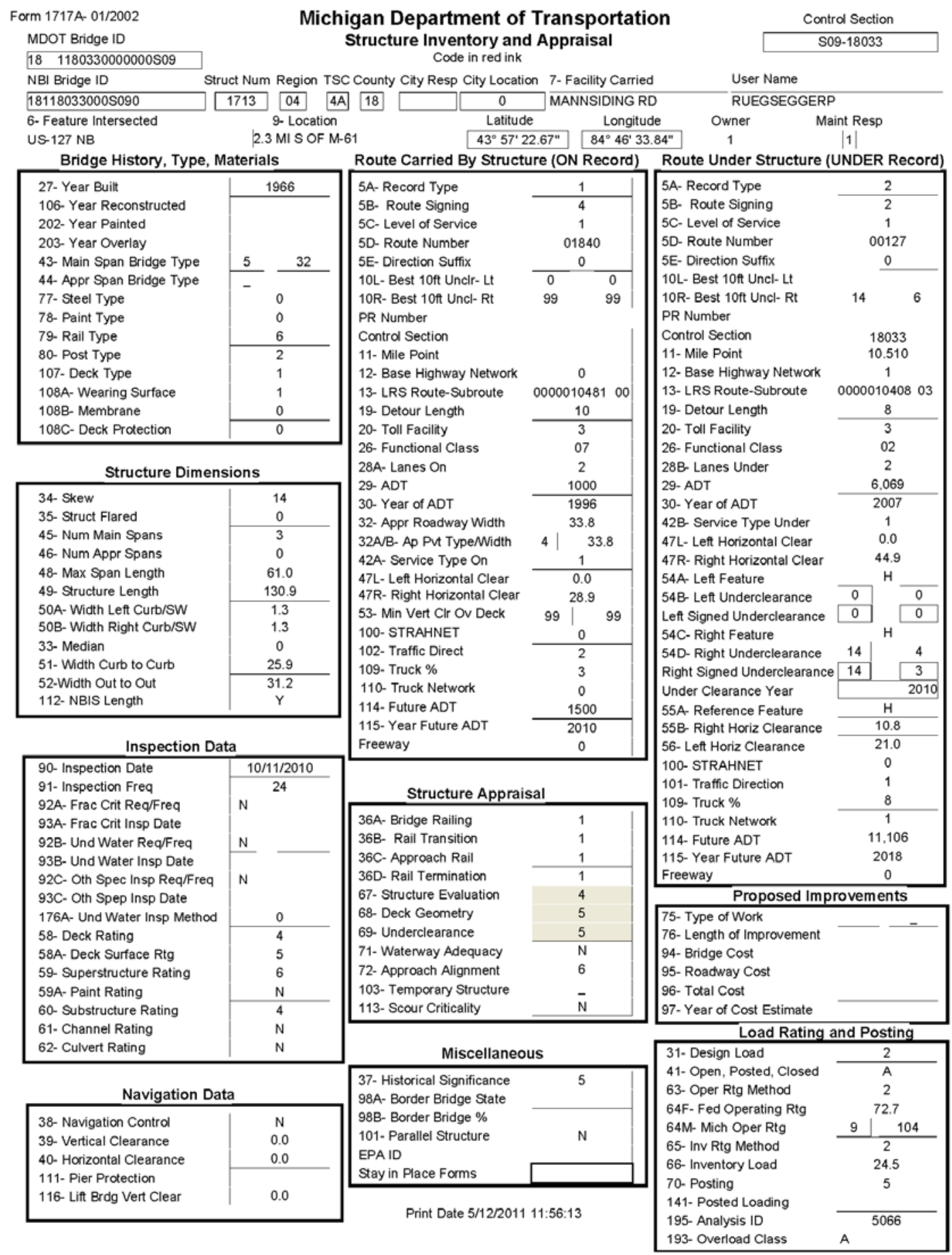

Figure A.1, continued. (MDOT 2011b) 


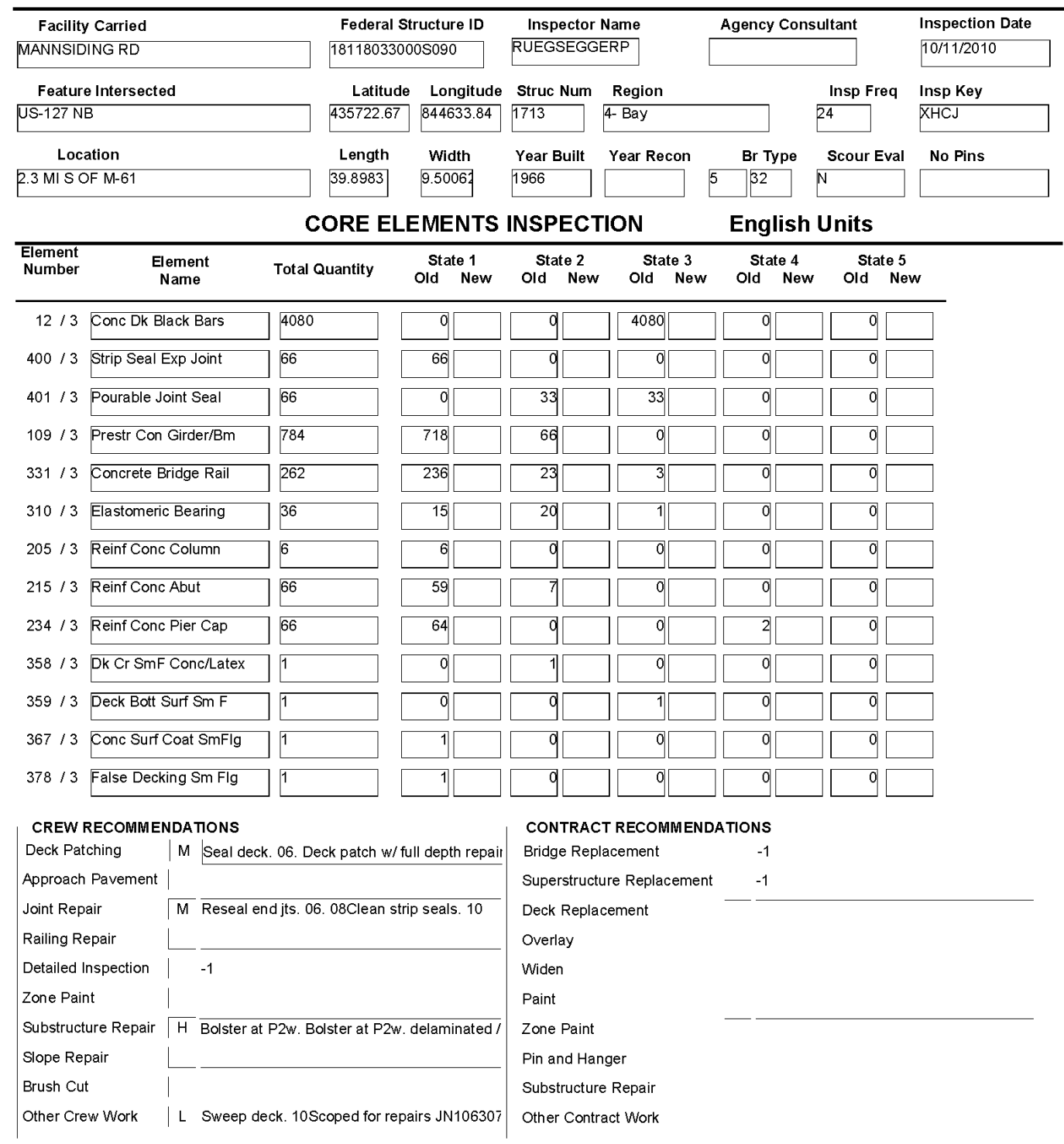

Figure A.1, continued. (MDOT 2011b) 


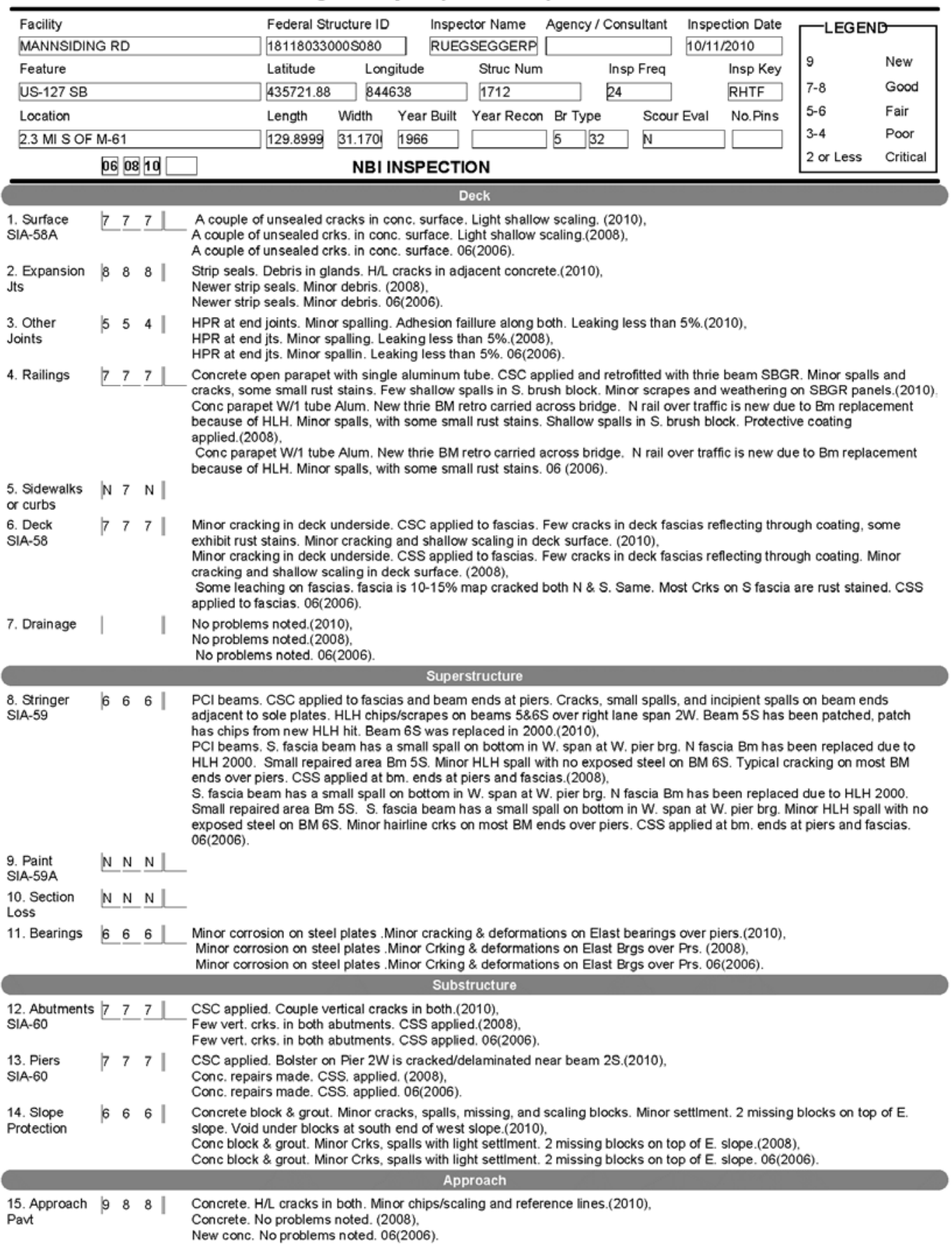

Figure A.2: Mannsiding Road SB routine bridge inspection report (MDOT 2011b) 
Michigan Department of Transportation Form P2502 (02/2002)

Bridge Safety Inspection Report

Page 2 of 2

Facility

Federal Structure ID Inspector Name Agency

S08-18033

Fection Date

\begin{tabular}{llllll}
\hline Feature & Latitude & Longitude & Struc Num & Insp Freq & Insp Key
\end{tabular}

US-127 SB

Location

$435721.88 \quad 844638$

Struc Num

Length Width Year Built Year Recon $\mathrm{Br}$ Type

24

RHTF 7-8

$2.3 \mathrm{MI} \mathrm{S}$ OF M-61

129.899931 .1701966

$0 6 \longdiv { 0 8 } 1 0 \square$ NBI INSPECTION

\begin{tabular}{|c|c|c|c|}
\hline $\begin{array}{l}\text { 16. Approach } \\
\text { Shldrs Swalk }\end{array}$ & $\mid \mathrm{N} N$ & $N \quad N$ & $\begin{array}{l}\text { Narrow Conc curb. (2008), } \\
\text { Narrow Conc curb. 06(2006). }\end{array}$ \\
\hline $\begin{array}{l}\text { 17. Approach } \\
\text { Slopes }\end{array}$ & & & $\begin{array}{l}\text { Vegetated. No problems noted. }(2010) \text {, } \\
\text { Well vegetated. }(2008) \text {, } \\
\text { Well veg. } 06(2006) \text {. }\end{array}$ \\
\hline 18. Utilities & & & \\
\hline $\begin{array}{l}\text { 19. Channel } \\
\text { SIA-61 }\end{array}$ & $\mathbb{N} N$ & $N \quad \mathrm{~N} \|$ & \\
\hline $\begin{array}{l}\text { 20. Drainage } \\
\text { Culverts }\end{array}$ & & & $\begin{array}{l}\text { Minor dirt and debris at NE. and SE. (2010), } \\
\text { Minor dirt and debris at NE. and SE. (2008), }\end{array}$ \\
\hline
\end{tabular}

Culverts Minor dirt and debris at NE. and SE. (2008),

Minor dirt and debris at NE. and SE. 06(2006).

\begin{tabular}{|c|c|c|c|c|c|c|c|c|c|c|}
\hline \multirow[t]{2}{*}{ Guard Rail } & \multicolumn{4}{|c|}{$\sqrt{2}$} & \multicolumn{3}{|c|}{ [Crit Feat Insp(S|A-92) } & \multirow{6}{*}{$\begin{array}{l}71 \quad \text { Watr Adeq } \\
72 \text { Appr Align } \\
\text { Temp Supp } \\
\text { Hi Ld Hit (M) } \\
\text { Special Insp Euip. }\end{array}$} & \multirow{3}{*}{6} & \multirow{6}{*}{$\begin{array}{l}\text { General Notes } \\
\begin{array}{l}\text { CSM Crk. seal. Reseal end jts. 06. Healer } \\
\text { sealer. } 08\end{array}\end{array}$} \\
\hline & 06 & 10 & 10 & & \multirow{5}{*}{$\begin{array}{ll}92 \mathrm{~A} & \text { Frac } \text { Crit } \\
92 \mathrm{~B} & \text { Und. Watr } \\
92 \mathrm{C} & \text { Spl.Insp }\end{array}$} & \multirow{2}{*}{\multicolumn{2}{|c|}{ Freq Date }} & & & \\
\hline $36 \mathrm{~A}$ & 1 & 1 & 1 & 1 & & & & & & \\
\hline $36 \mathrm{~B}$ & 1 & 1 & 1 & 1 & & & & & & \\
\hline $36 \mathrm{C}$ & 1 & 1 & 1 & 1 & & & & & & \\
\hline $36 \mathrm{D}$ & 1 & 1 & 1 & 1 & & & & & & \\
\hline
\end{tabular}

Figure A.2, continued. (MDOT 2011b) 


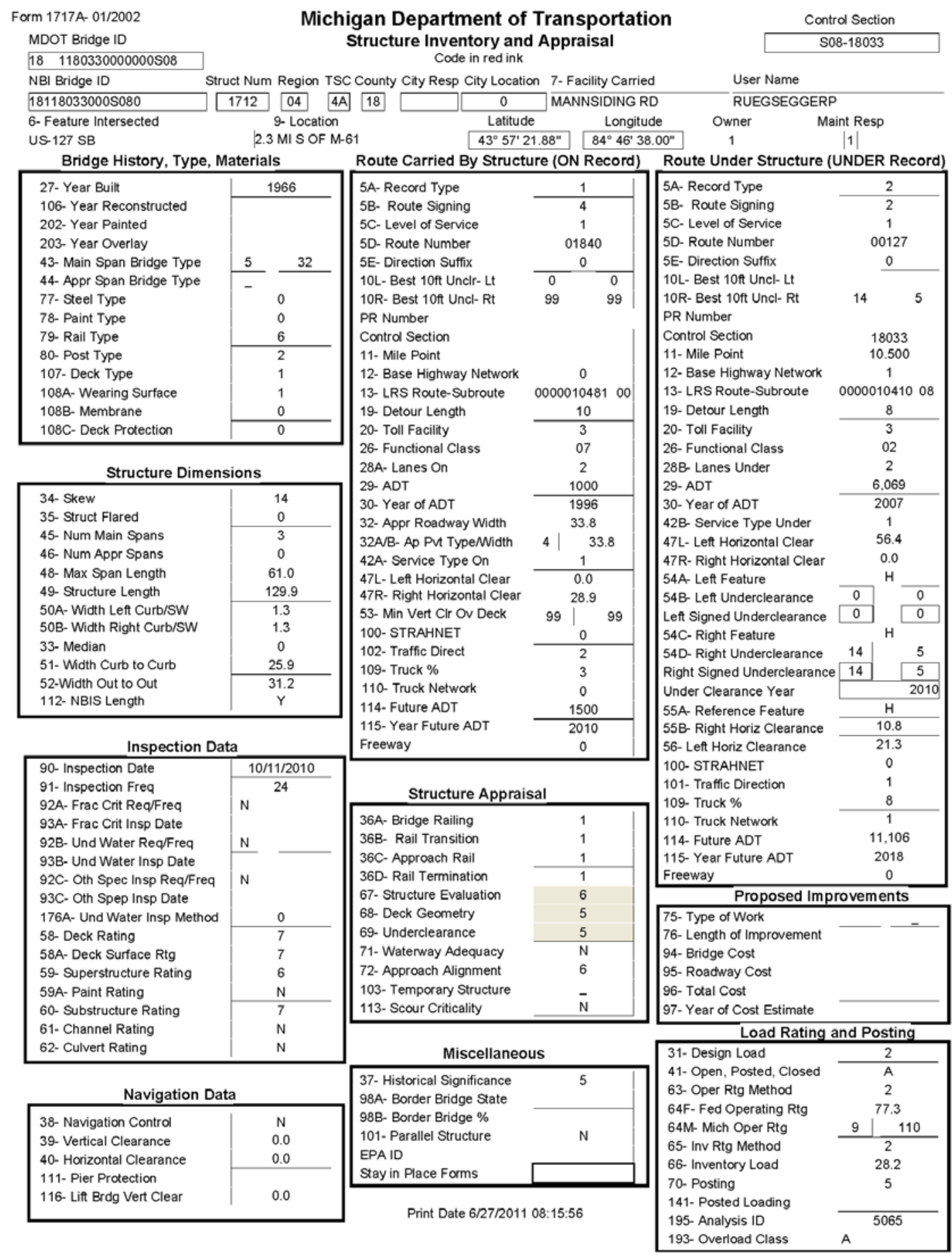

Figure A.2, continued. (MDOT 2011b) 


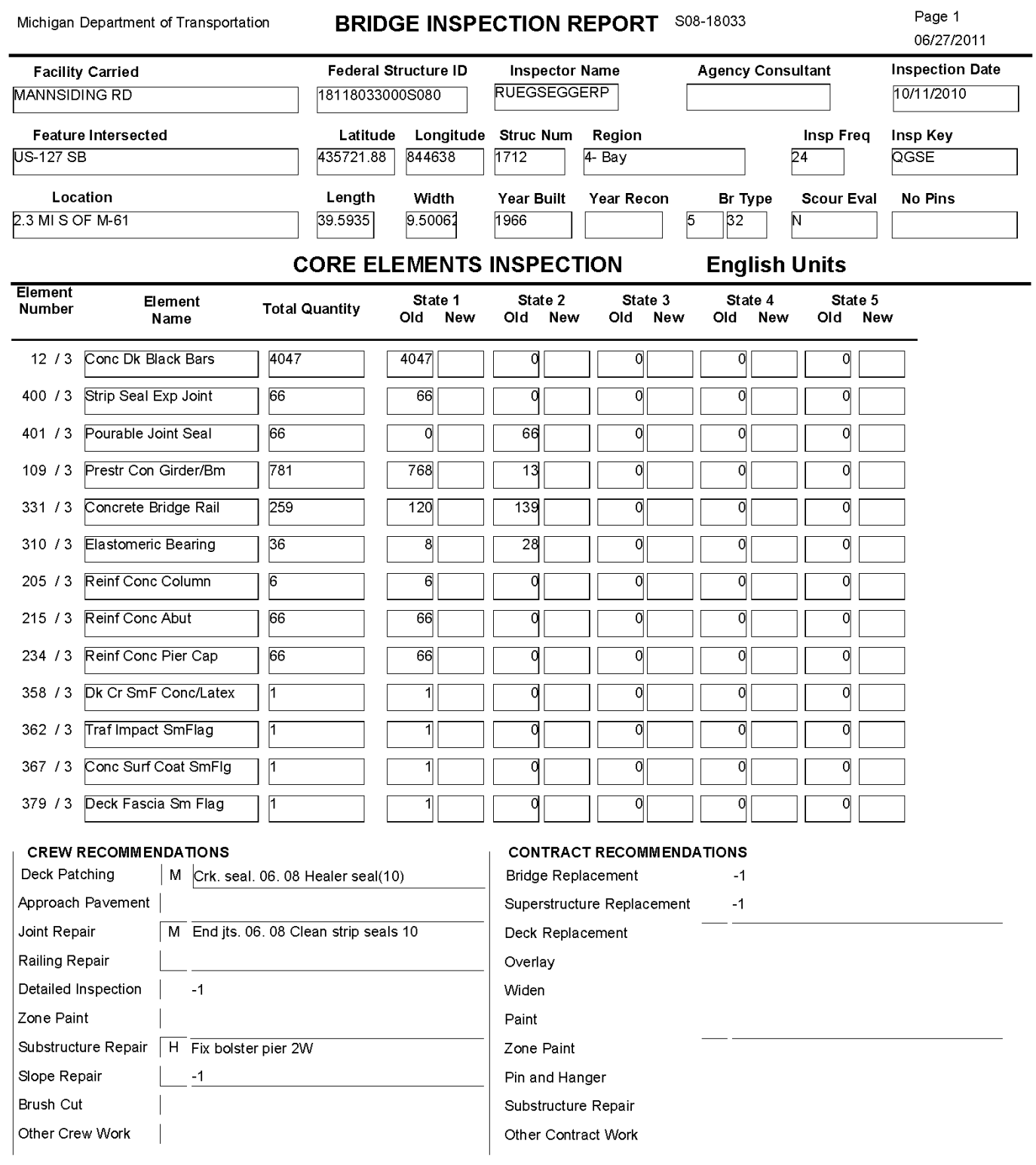

Figure A.2, continued. (MDOT 2011b) 


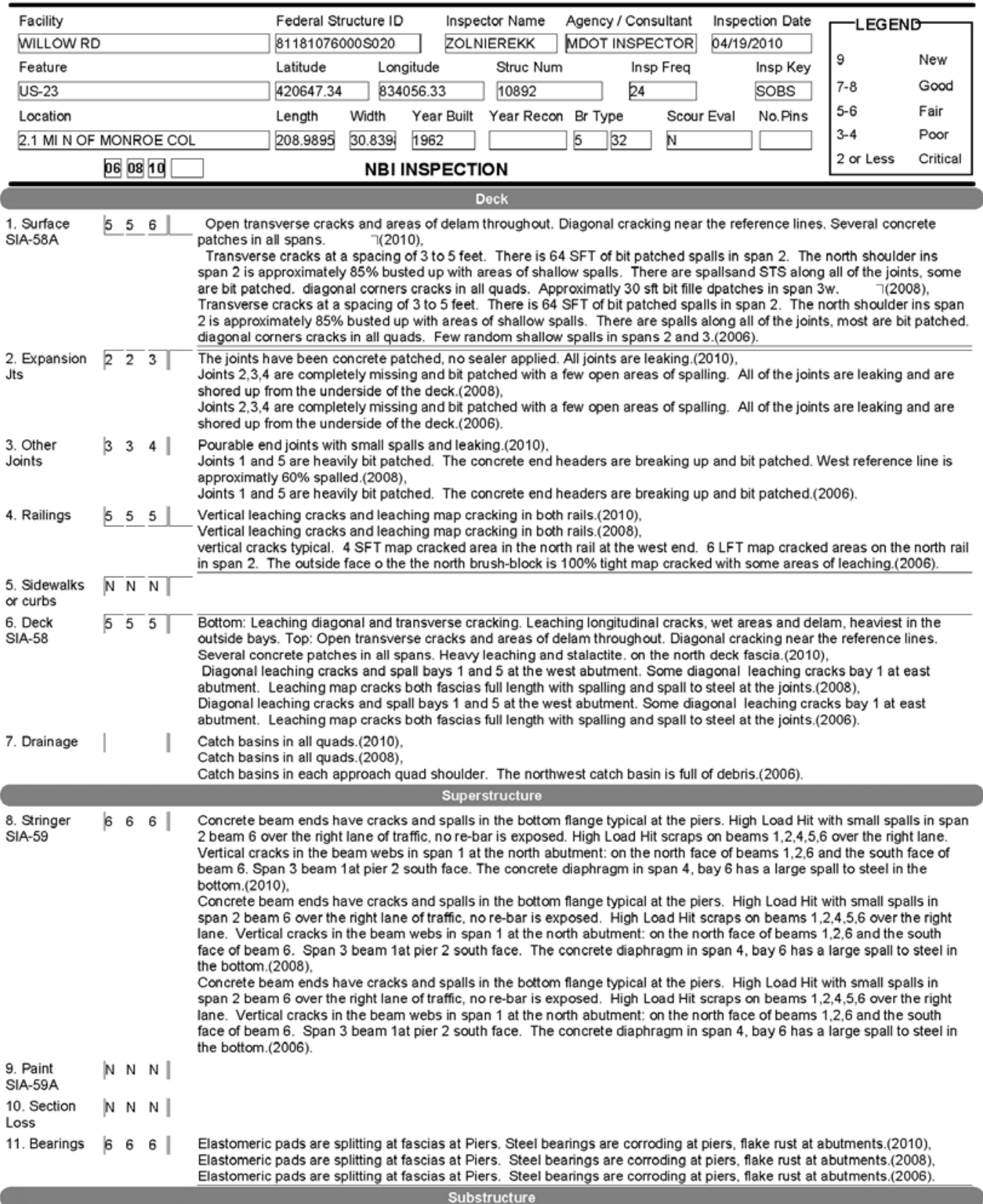
Substructure

\section{Figure A.3: Willow Road routine bridge inspection report (MDOT 2011b)}




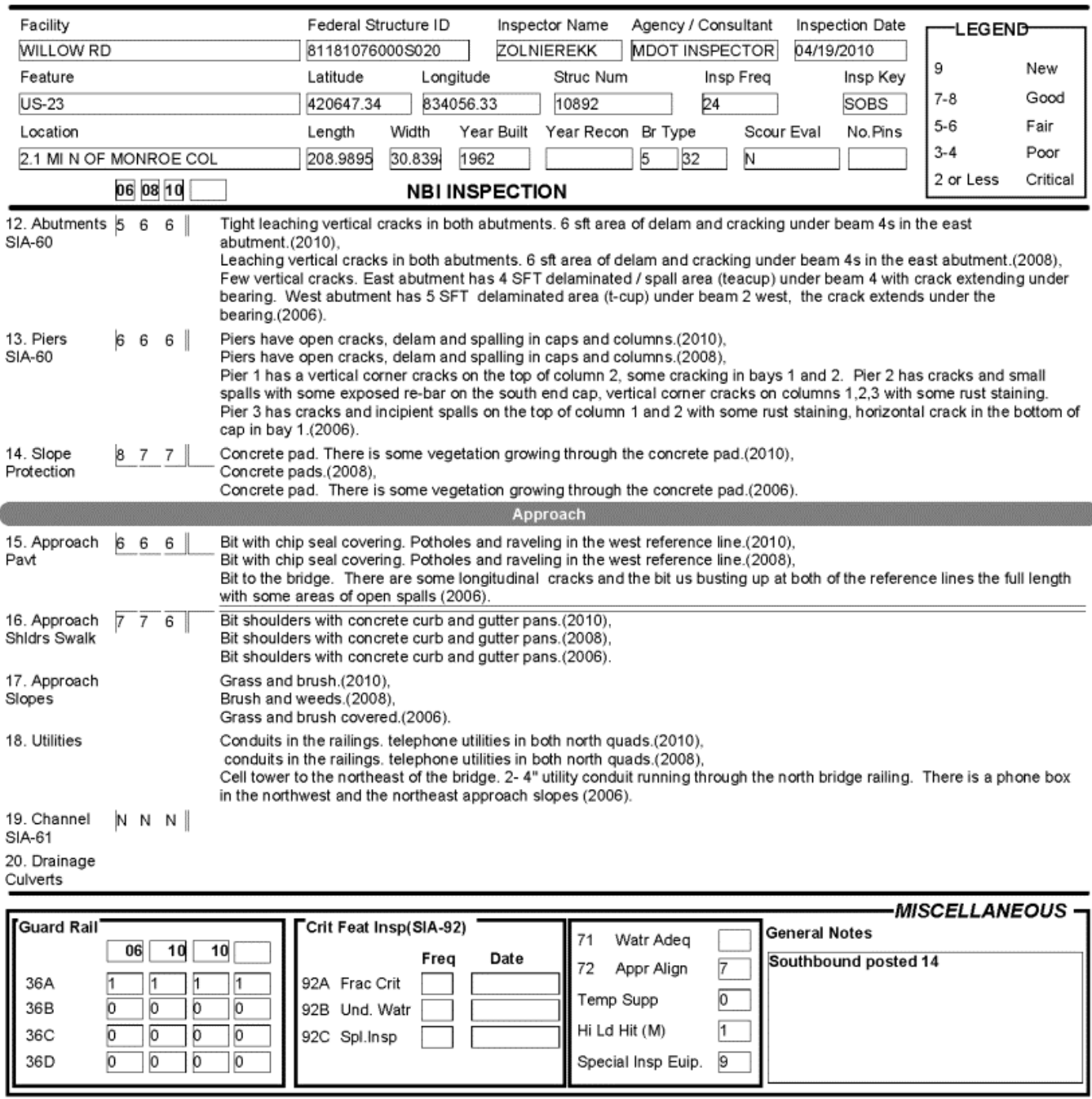

Figure A.3, continued. (MDOT 2011b) 


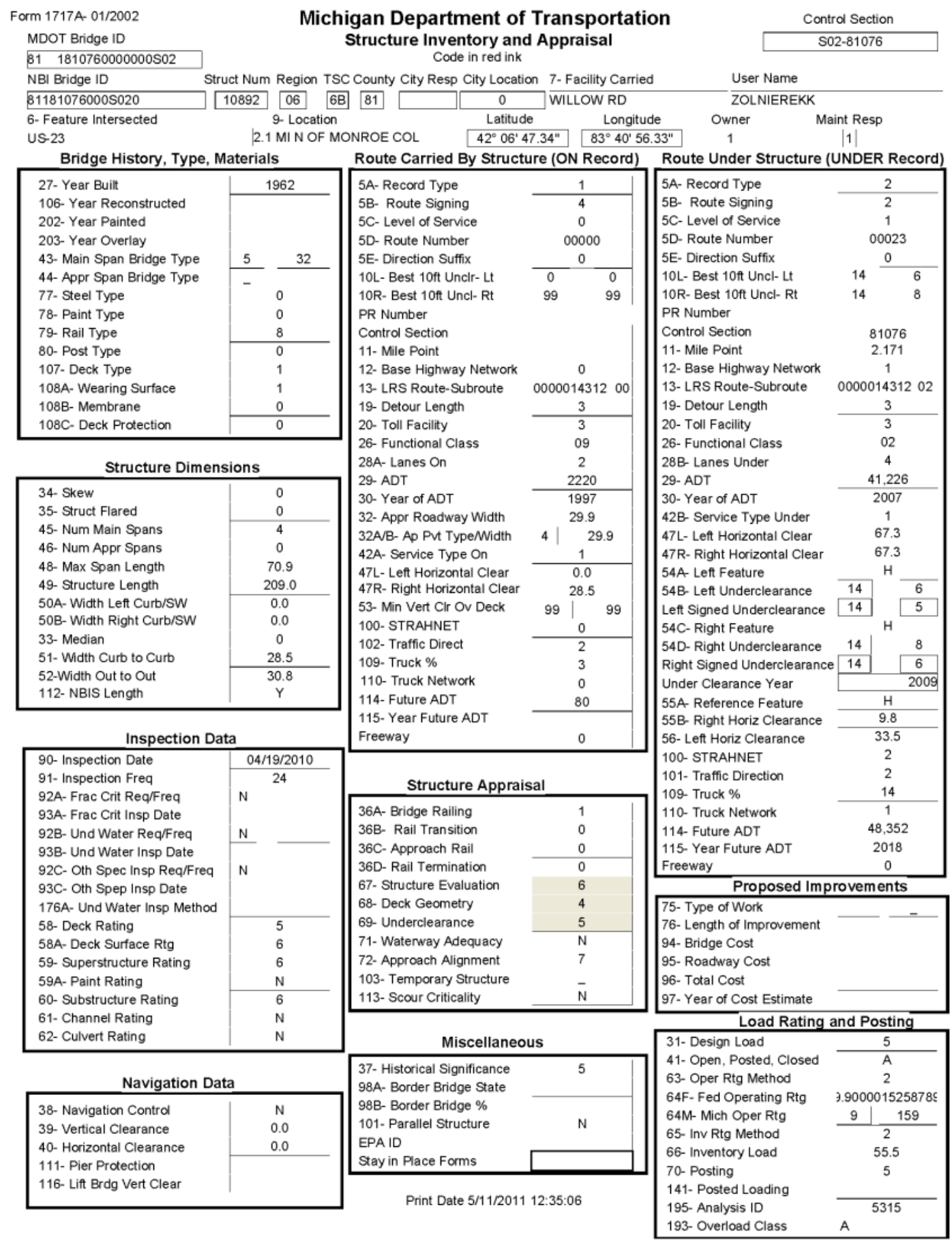

Figure A.3, continued. (MDOT 2011b) 


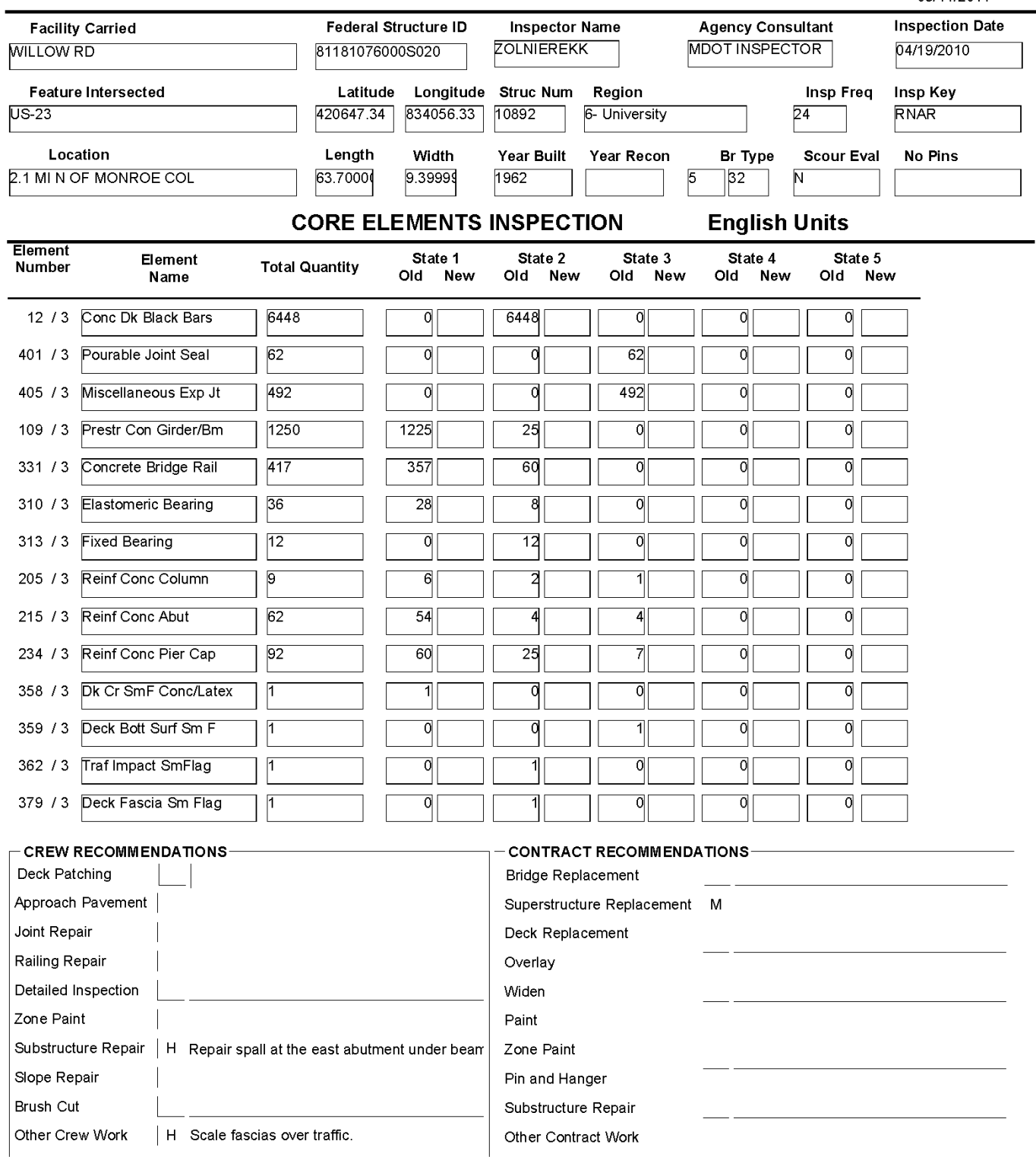

Figure A.3, continued. (MDOT 2011b) 


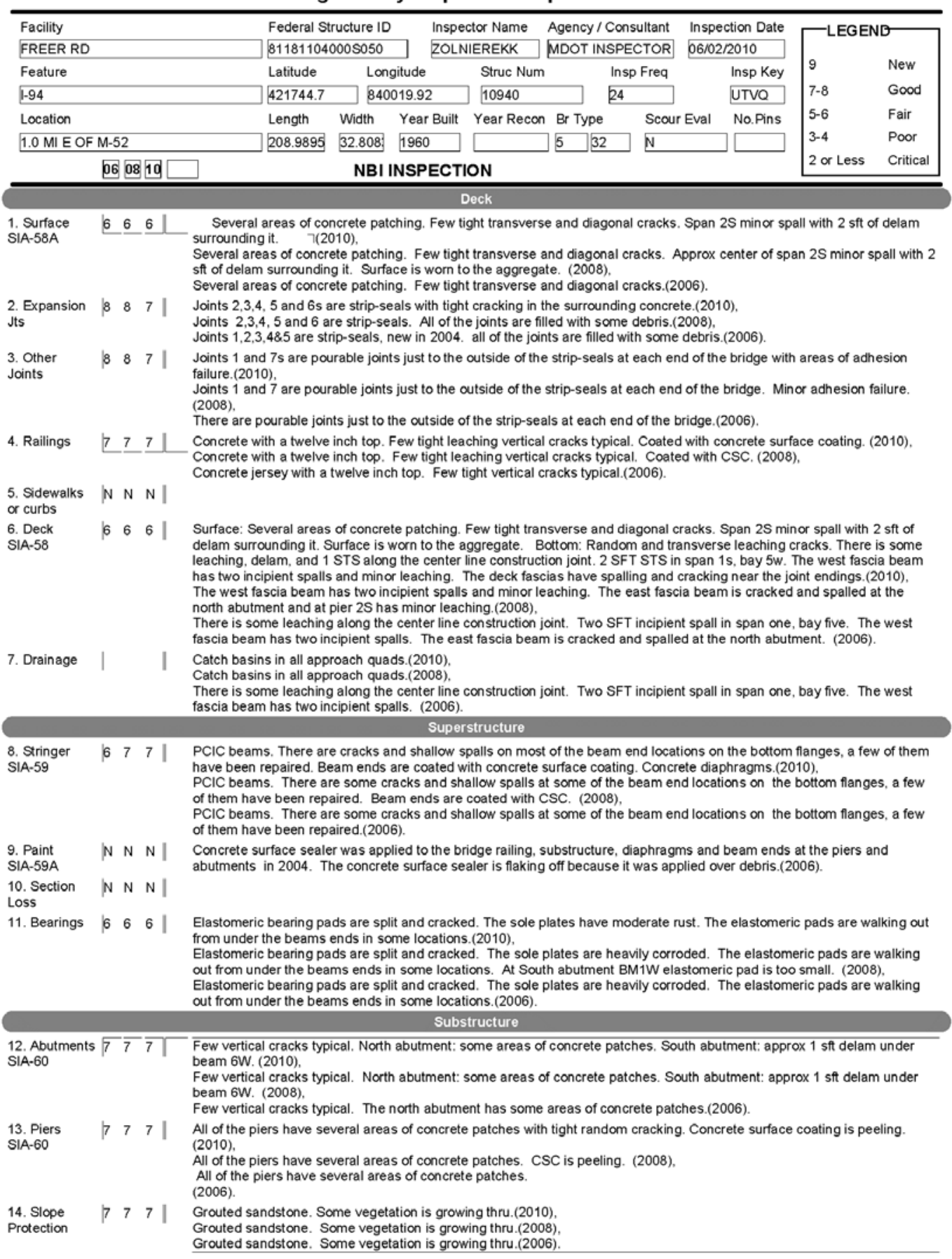

Figure A.4: Freer Road routine bridge inspection report (MDOT 2011b) 


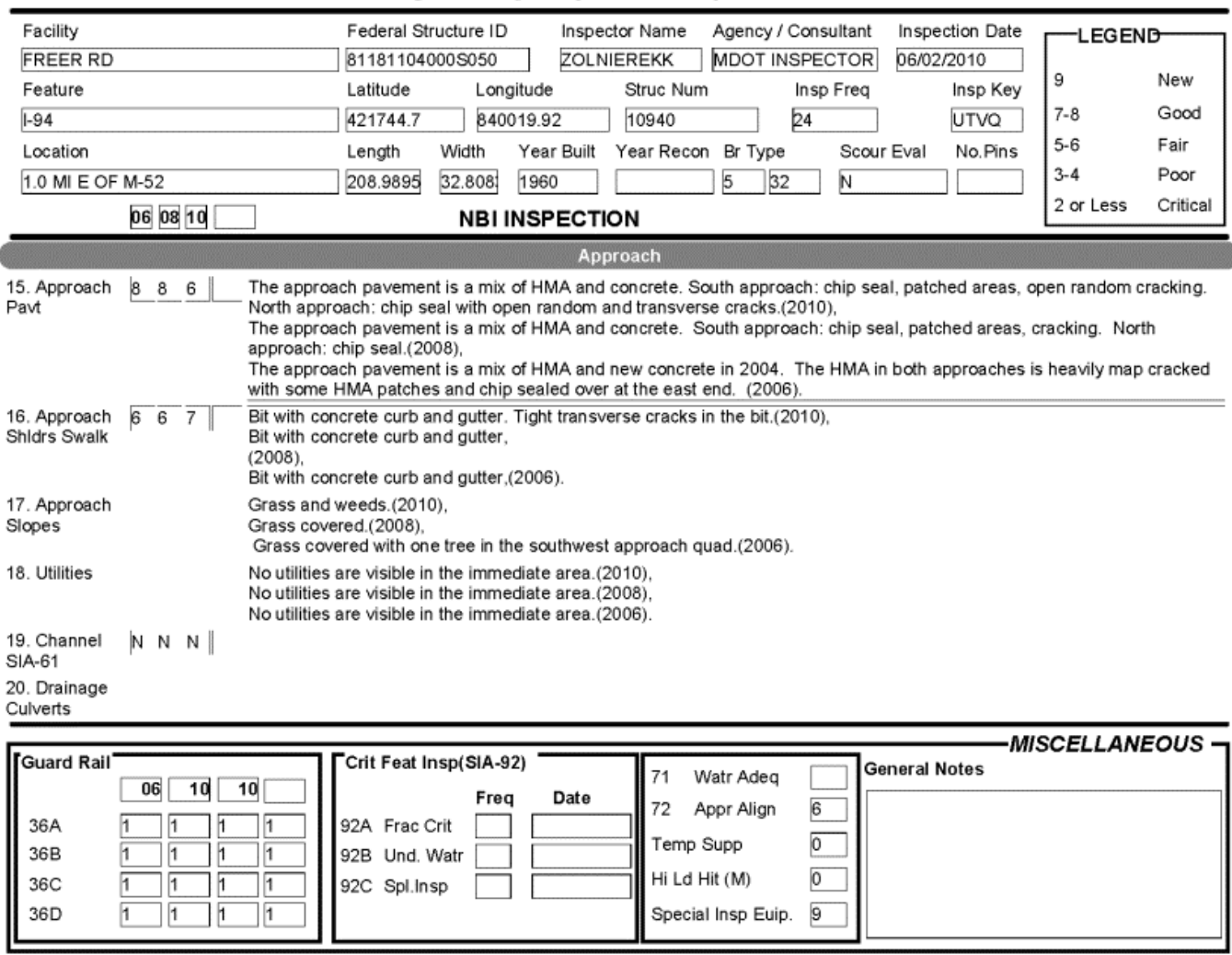

Figure A.4, continued. (MDOT 2011b) 


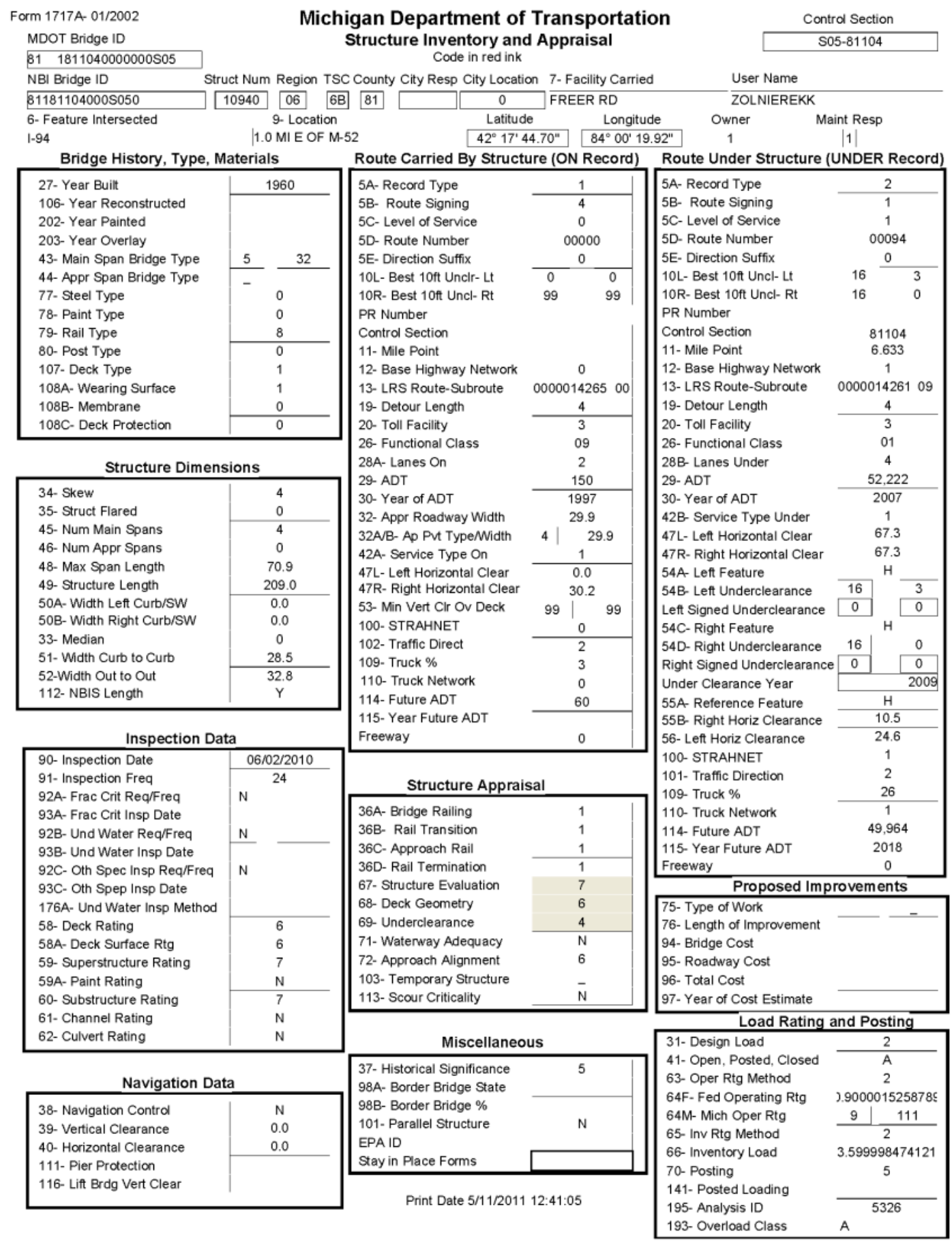

Figure A.4, continued. (MDOT 2011b) 
Michigan Department of Transportation

Facility Carried
FREER RD
Feature Intersected
$1-94$
Location
$1.0 \mathrm{MI}$ E OF M-52

BRIDGE INSPECTION REPORT S05-81104

\begin{tabular}{|c|c|c|c|c|c|c|}
\hline \multirow{2}{*}{\multicolumn{2}{|c|}{$\begin{array}{l}\text { Federal Structure ID } \\
81181104000 \$ 050\end{array}$}} & \multirow{2}{*}{\multicolumn{2}{|c|}{$\begin{array}{r}\text { Inspector Name } \\
\text { ZOLNIEREKK }\end{array}$}} & \multirow{2}{*}{\multicolumn{2}{|c|}{$\begin{array}{l}\text { Agency Consultant } \\
\text { MDOT INSPECTOR }\end{array}$}} & \multirow{2}{*}{$\begin{array}{l}\text { Inspection Date } \\
06 / 02 / 2010\end{array}$} \\
\hline & & & & & & \\
\hline Latitude & Longitude & Struc Num & Region & & Insp Freq & Insp Key \\
\hline 421744.7 & 840019.92 & 10940 & 6- University & & 24 & TSUP \\
\hline Length & Width & Year Built & Year Recon & Br Type & Scour Eval & No Pins \\
\hline 63.7000 & 10 & 1960 & & 32 & $\mathbb{N}$ & \\
\hline
\end{tabular}

\section{CORE ELEMENTS INSPECTION}

\begin{tabular}{|c|c|c|}
\hline $\begin{array}{l}\text { Element } \\
\text { Number }\end{array}$ & $\begin{array}{l}\text { Element } \\
\text { Name }\end{array}$ & Total Quantity \\
\hline $12 / 3$ & Conc Dk Black Bars & 6878 \\
\hline $400 / 3$ & Strip Seal Exp Joint & 165 \\
\hline $401 / 3$ & Pourable Joint Seal & 66 \\
\hline $109 / 3$ & Prestr Con Girder/Bm & 1253 \\
\hline $331 / 3$ & Concrete Bridge Rail & 417 \\
\hline $310 / 3$ & Elastomeric Bearing & 48 \\
\hline $205 / 3$ & Reinf Conc Column & 9 \\
\hline $215 / 3$ & Reinf Conc Abut & 66 \\
\hline $234 / 3$ & Reinf Conc Pier Cap & 102 \\
\hline $359 / 3$ & Deck Bott Surf Sm F & 1 \\
\hline $367 / 3$ & Conc Surf Coat SmFlg & 1 \\
\hline $379 / 3$ & Deck Fascia Sm Flag & 1 \\
\hline
\end{tabular}

\begin{tabular}{ccccccc}
\multicolumn{2}{c}{ State 1} & \multicolumn{2}{c}{ State 2} & \multicolumn{2}{c}{ State 3} & \multicolumn{2}{c}{ State 4} & \multicolumn{2}{c}{ State 5} \\
Old New & Old & New & Old New & Old New & Old New
\end{tabular}

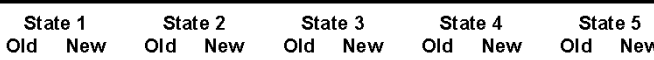

English Units

Page 1

$$
05 / 11 / 2011
$$

\begin{tabular}{|c|c|c|c|c|}
\hline 0 & 6878 & 0 & 0 & 0 \\
\hline 165 & 9 & 0 & 0 & 0 \\
\hline 0 & 66 & 0 & 0 & 0 \\
\hline 1205 & 48 & 0 & 0 & 0 \\
\hline 397 & 20 & 0 & 0 & 0 \\
\hline 32 & 16 & 0 & 0 & 0 \\
\hline 9 & 9 & 0 & 0 & 0 \\
\hline 58 & 8 & 0 & 0 & 0 \\
\hline 98 & 4 & 0 & 0 & 0 \\
\hline 0 & 1 & 0 & 0 & 0 \\
\hline 0 & 1 & 0 & 0 & 0 \\
\hline 1 & 9 & 0 & 0 & 0 \\
\hline
\end{tabular}

\section{CREW RECOMMENDATIONS}

Deck Patching $|\mathrm{L}| 2$ sft patch in center of span $2 \mathrm{~S}$.

Approach Pavement

Joint Repair

Railing Repair

Detailed Inspection

Zone Paint

Substructure Repair

Slope Repair

Brush Cut

Other Crew Work $\quad \mathrm{H}$ Scale span $2 \mathrm{~S}$ bay $3 \mathrm{~W}$, span $3 \mathrm{~S}$ bay $3 \mathrm{~W}$, anc
CONTRACT RECOMMENDATIONS

Bridge Replacement

Deck Replacement

Overlay M Epoxy overlay

Widen

Paint

Zone Paint

Pin and Hanger

Substructure Repair

Other Contract Work
Superstructure Replacement

Figure A.4, continued. (MDOT 2011b) 


\section{Appendix B: Leica ScanStation C10 Field Sketches}

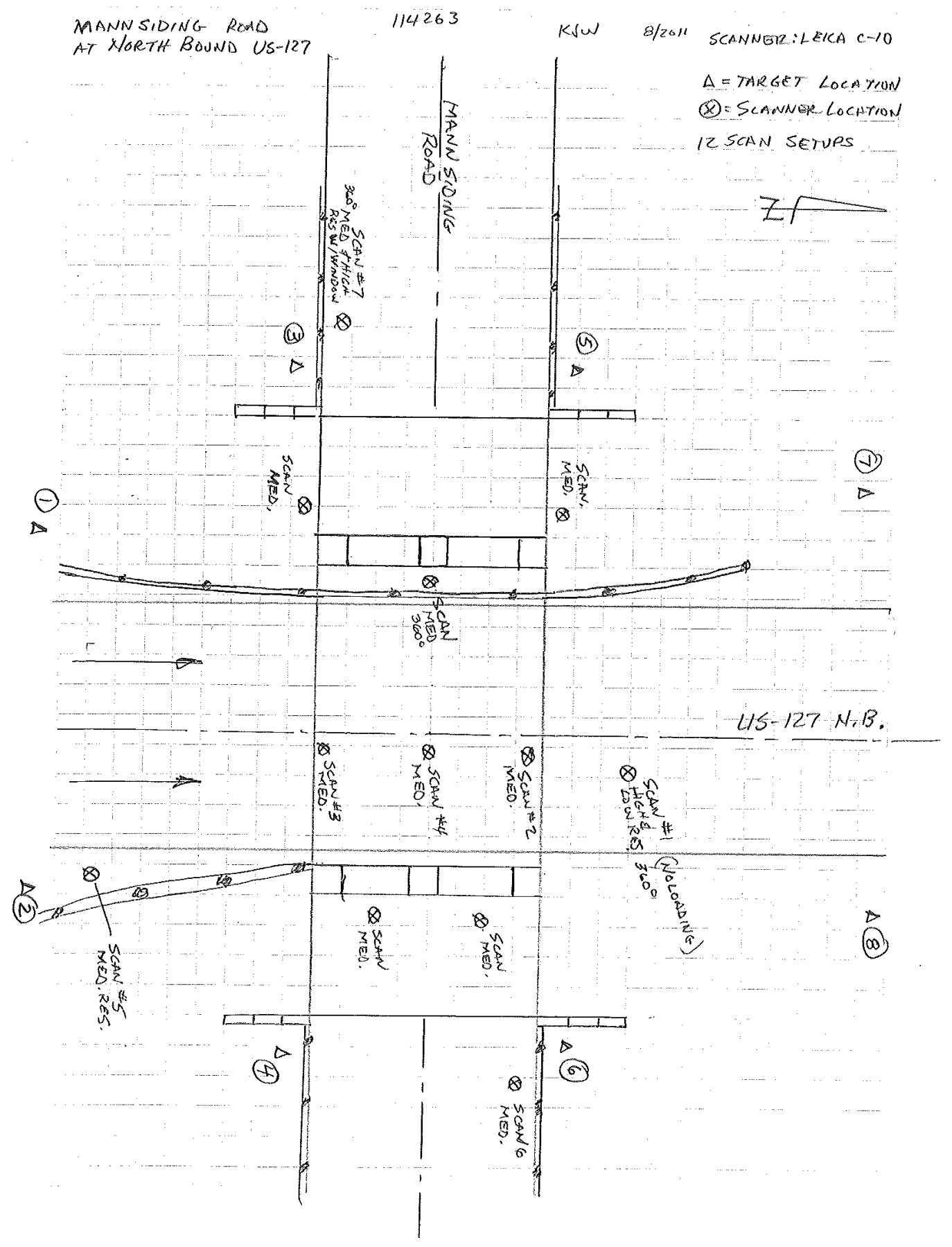

Figure B.1: Mannsiding Road NB Leica ScanStation C10 collection locations 


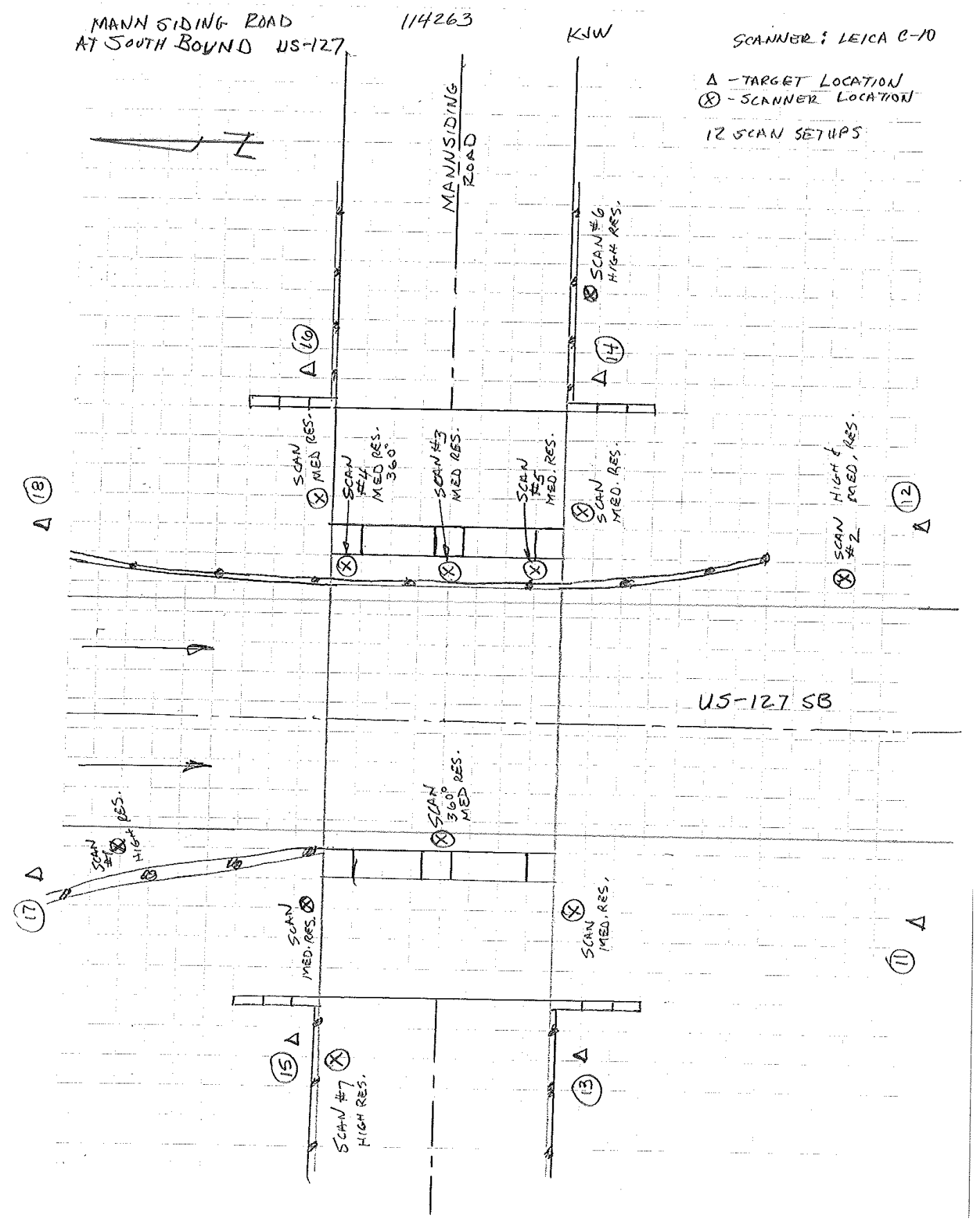

Figure B.2: Mannsiding Road SB Leica ScanStation C10 collection locations 


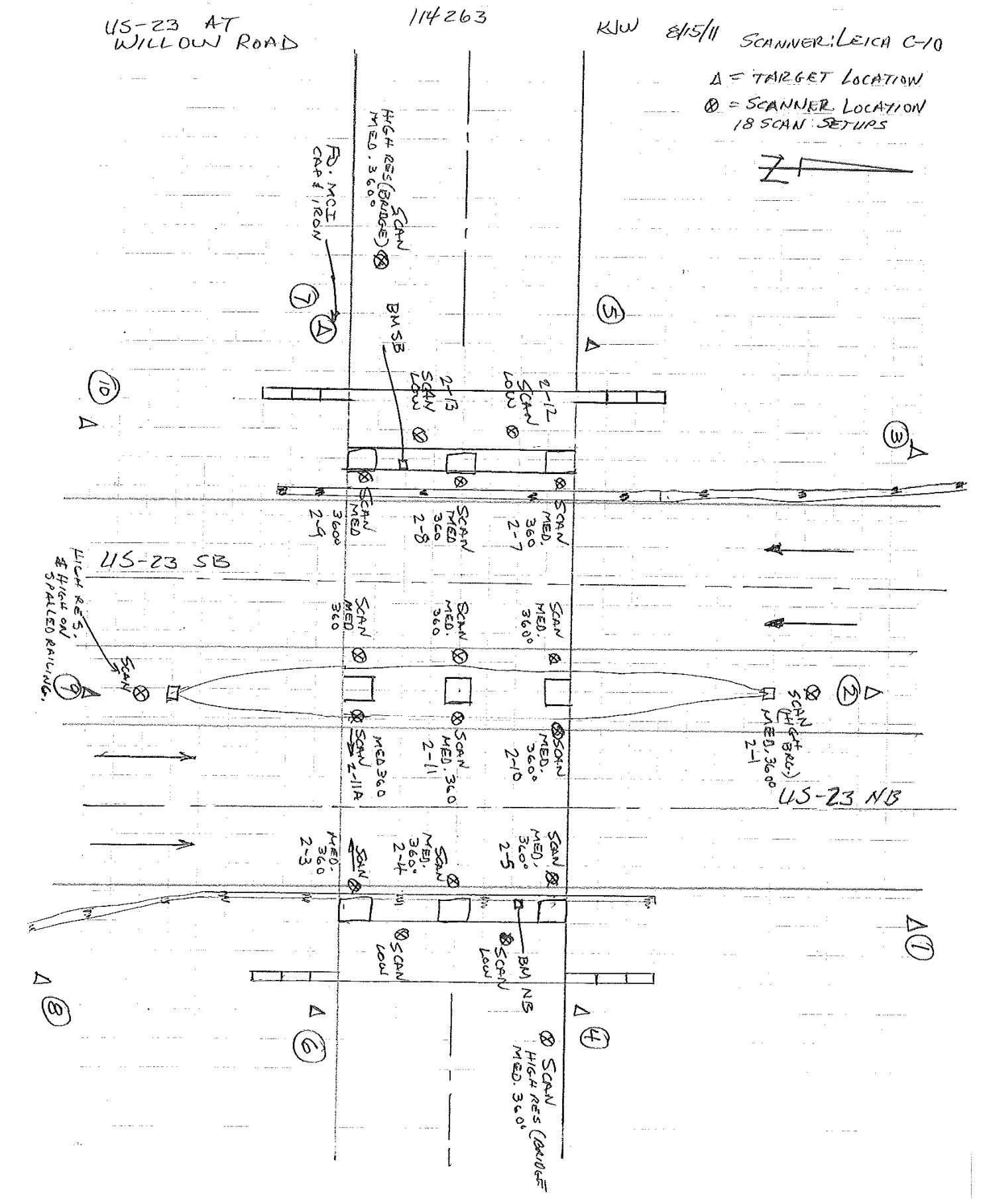

Figure B.3: Willow Road Leica ScanStation C10 collection locations 


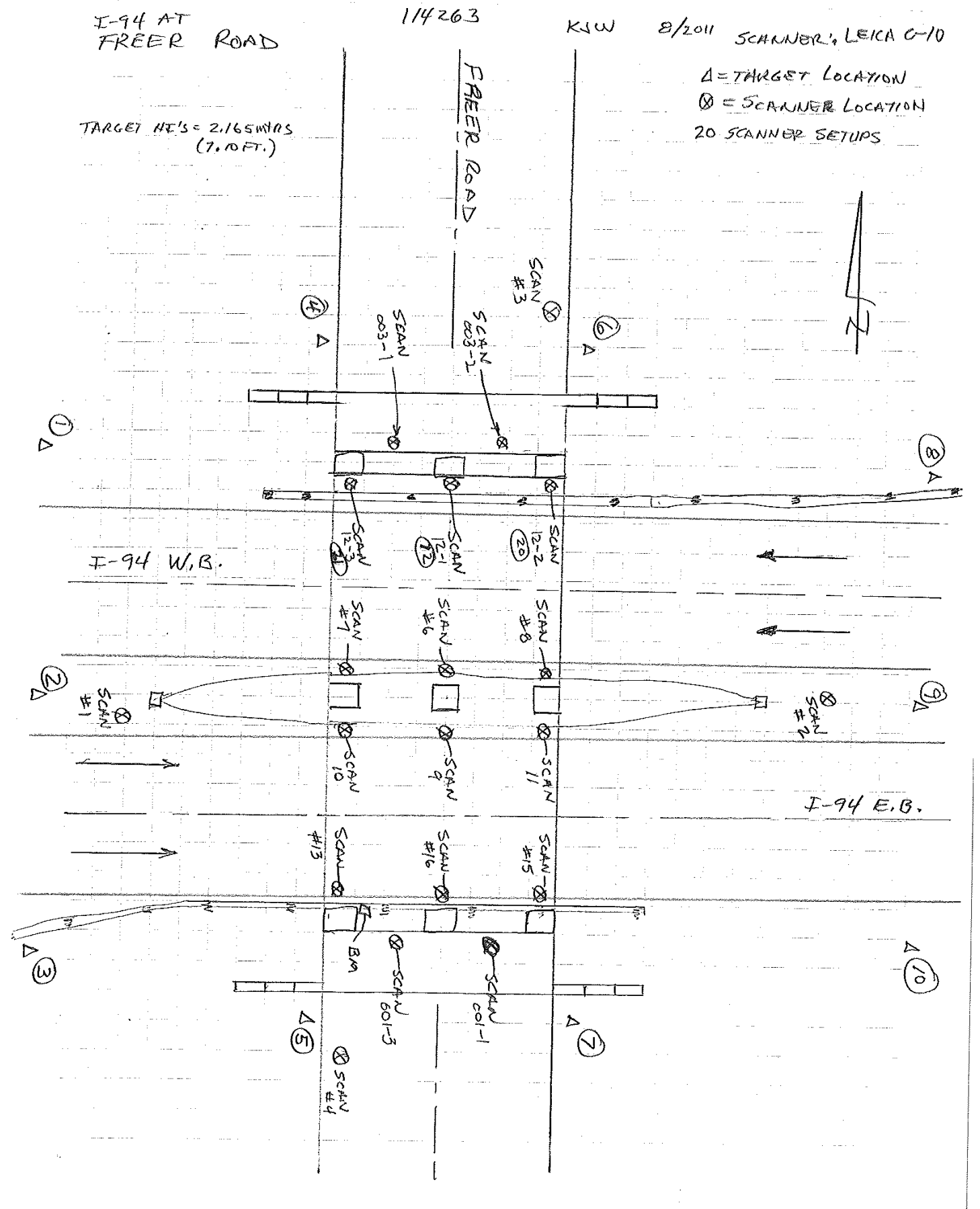

Figure B.4: Freer Road Leica ScanStation C10 collection locations 


\section{Appendix C: Algorithm Results}

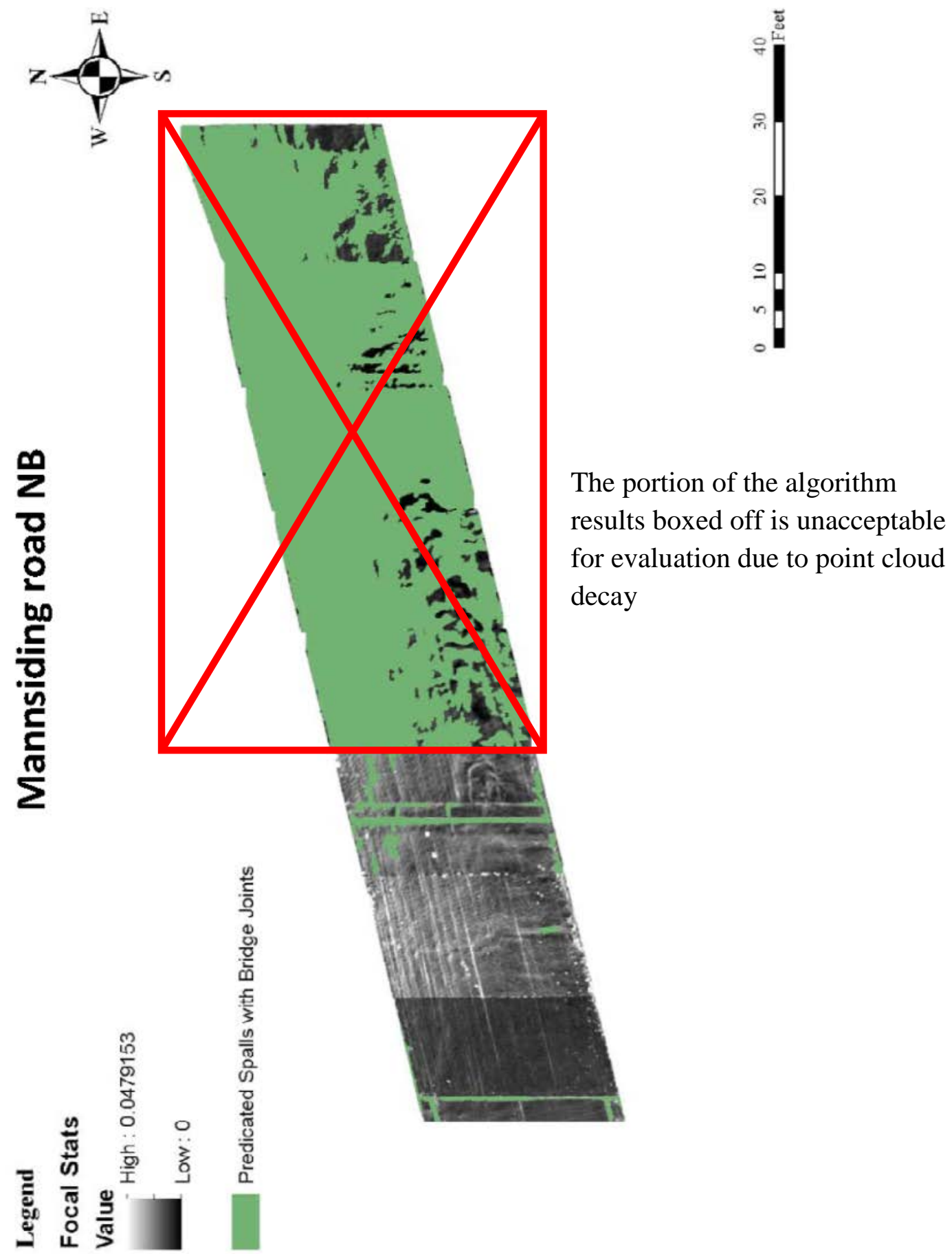

Figure C.1: Mannsiding Road NB spall algorithm results at established input parameters 


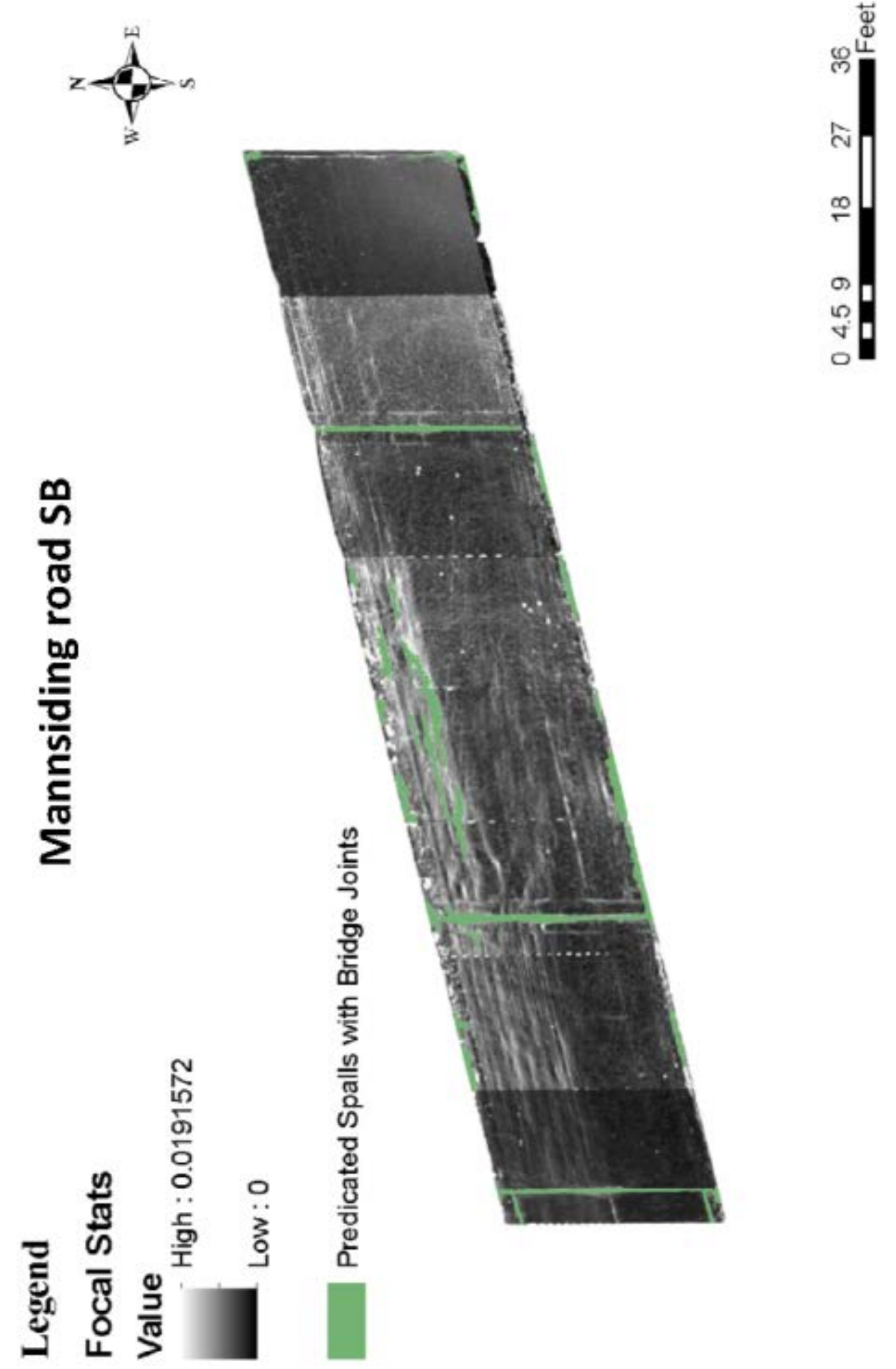

Figure C.2: Mannsiding Road SB spall algorithm results at established input parameters 

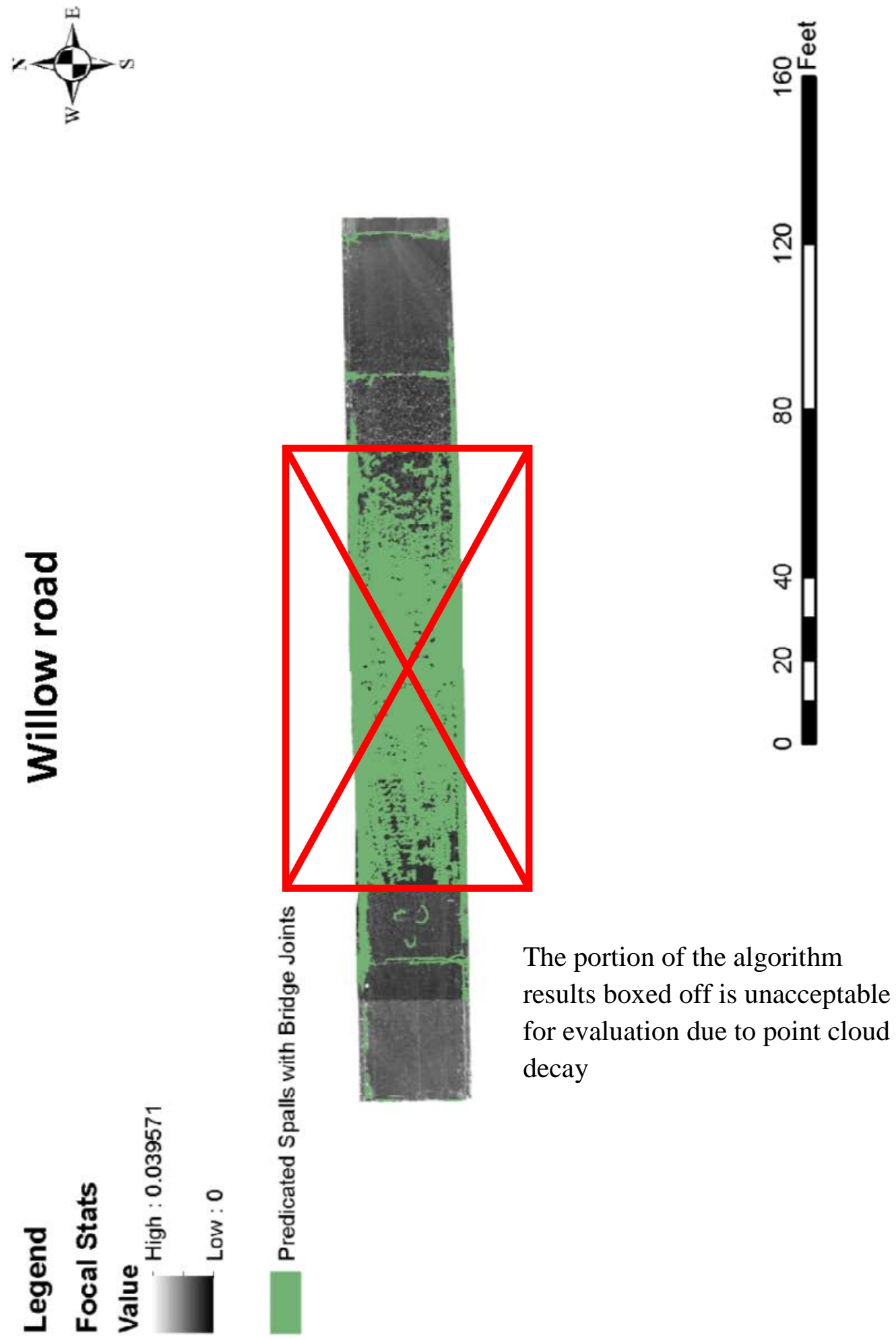

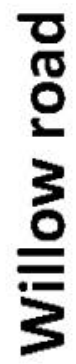

Figure C.3: Willow Road spall algorithm results at established input parameters 

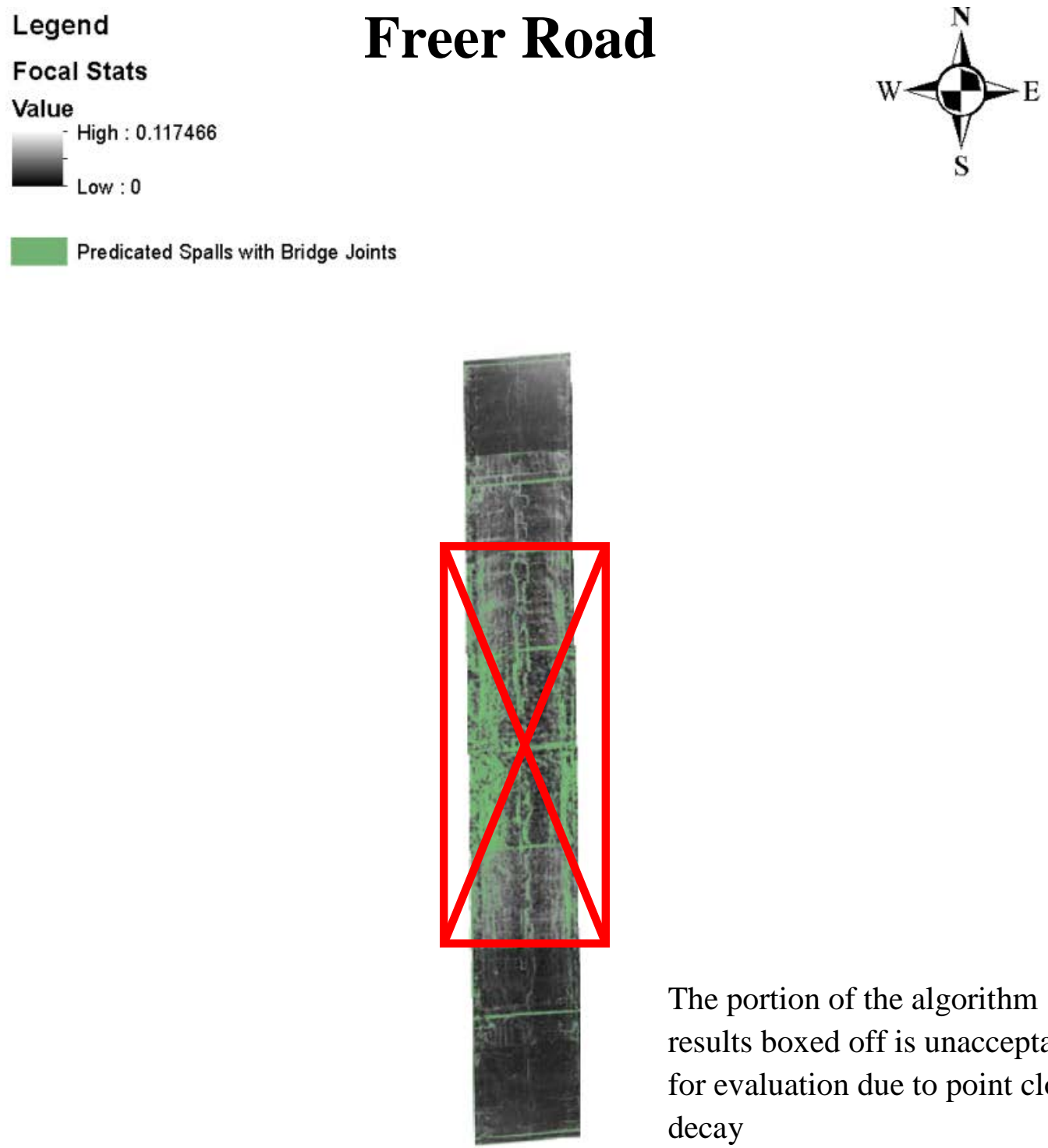

The portion of the algorithm results boxed off is unacceptable for evaluation due to point cloud decay

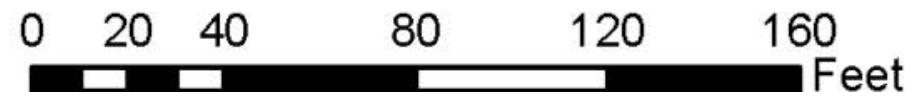

Figure C.4: Freer Road spall algorithm results at established input parameters 


\section{Appendix D: Clarification of Potential Copyrighted Material}

The following materials were generated through the RITA research project, which is the project my thesis is being funded under. Release of the copyrights have been obtained in writing and can be viewed on pages 135 to 137 . This applies to the following figures:

2.4, 2.5, 2.6, 2.7, 2.9, 3.1, 3.2, 3.5, 3.7, 3.9, 3.11, 3.12, 3.14, 4.11, 4.12, 4.13 and 4.14

The following material is considered public domain. The RITA research project worked closely with the USDOT and MDOT. Documents and figures have been provided to the team to enhance the published work. This applies to the following areas:

\section{1, 2.3, appendix $A$ and $B$}

The following materials were obtained through the use of "free trial" versions of processing software and are considered "fair use" material. The figures are:

\subsection{Through 3.25 from Certainty 3D}

\subsection{Through 3.31 from Quick Terrain}

The following materials have been released by the author, allowing for the repurposing of the material in the thesis. Documentation of the release of rights can be seen on pages 138-140. This applies to the following areas:

Figure 2.12, 2.15 and Table 2.5.

Image Quality:

Screenshots and low quality images are presented within the thesis. Figures, which are comprised of screenshots, have a resolution great than $72 \mathrm{dpi}$. The low quality images that are not screenshots were obtained from MTRI, a downstate affiliate, working on the RITA project and the obtained quality was the highest available. This applies to the following areas:

Screenshots:

Figures2.15, 3.3, 3.4, 3.6, 3.8, 3.10, 3.13, 3.15, 3.16, 3.17, 3.18, 3.19, 3.20, 3.21, 3.22, 3.23, 3.24, 3.25, 3.26, 3.27, 3.28, 3.29, 3.30, 3.31, 3.32, 3.33 and Tables 2.5, 3.3

Low quality images:

Figures 3.5, 3.7, 3.9 
Michigan Technological University Mail - Authority release of copyrighte... https://mail.google.com/mail/w/0/?ui=2\&ik=40b9a11e7d\&view=pt\&searc..

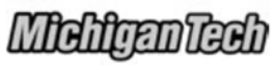

Ryan Hoensheid <rchoensh@mtu.edu>

\author{
Authority release of copyrighted material \\ 2 messages \\ Ryan Hoensheid <rchoensh@mtu.edu> \\ To: Khatereh Vaghefi <kvaghefi@mtu.edu> \\ Dear Khatereh, \\ Today I meet with an individual at the graduate school regarding copyrighted material and how to provide proof the \\ rights had been released to me. The photographs that you provided from the field demonstration are considered \\ copyrighted material that need to be released for use. The following figures are photographs provided by you and \\ have been incorporated into the body on my thesis: \\ 2.9 \\ I have attached a PDF of my thesis, so you can view the use of the material. All credit will be given to you \\ (Khatereh Vaghefi) and an appendix will be included in the final thesis stating the release of copyright, if given. \\ Could I use the material listed above? \\ Thank you \\ -Ryan Hoensheid \\ 겅 rchthes.pdf \\ 9604K
}

Thu, Apr 19, 2012 at 10:22 AM

Khatereh Vaghefi <kvaghefi@mtu.edu >

Thu, Apr 19, 2012 at 10:55 AM

To: Ryan Hoensheid <rchoensh@mtu.edu>

Yes, It's fine with me.

-Khatereh

[Quoted text hidden]

--

Khatereh Vaghefi

Graduate Research Assistant ( $\mathrm{PhD}$ Candidate)

Department of Civil and Environmental Engineering

Michigan Technological University

829 Dow Building

1400 Townsend Drive

Houghton, MI 49931 


\author{
Authority requested for release of copyright \\ 2 messages \\ Ryan Hoensheid <rchoensh@mtu.edu> \\ To: "Renee O." <roats@mtu.edu> \\ Thu, Apr 19, 2012 at 10:20 AM \\ Dear Renee, \\ Today I meet with an individual at the graduate school regarding copyrighted material and how to provide proof the \\ rights had been released to me. The photographs that you provided from the field demonstration are considered \\ copyrighted material that need to be released for use. The following figures are photographs provided by you and \\ have been incorporated into the body on my thesis:
}

$2.4,2.5,2.6,2.7,3.1,3.11,4.11$

I have attached a PDF of my thesis, so you can view the use of the material. All credit will be given to you (Renee Oats) and an appendix will be included in the final thesis stating the release of copyright, if given. Could I use the material listed above?

Thank you

-Ryan Hoensheid

$\Rightarrow$ rchthes.pdf

$9604 \mathrm{~K}$

Renee Oats <roats@mtu.edu>

Thu, Apr 19, 2012 at 11:04 AM

To: Ryan Hoensheid <rchoensh@mtu.edu>

Hi Ryan,

Yes, you can use those photographs for your thesis document. It is fine by me if you incorporate them into the body of your thesis.

Thanks,

Renee

[Quoted text hidden] 


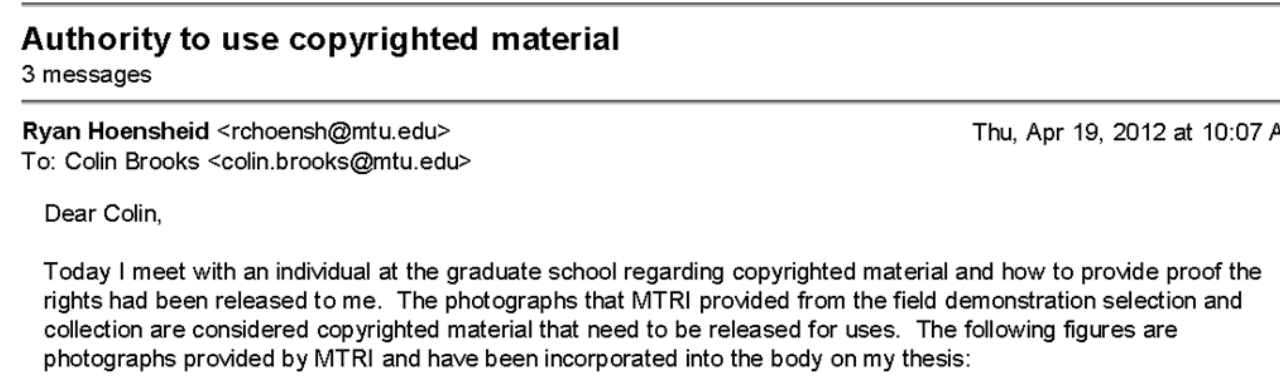

\section{$3.1,3.5,3.7,3.9,3.12,3.14,4.12,4.13,4.14$}

All credit will be given to MTRI and an appendix will be included in the final thesis stating the release of copyright, if given. Could I use the material listed above?

Thank you

-Ryan Hoensheid

Ryan Hoensheid <rchoensh@mtu.edu>

Mon, Apr 23, 2012 at 10:10 AM

To: Colin Brooks <colin.brooks@mtu.edu>

[Quoted text hidden]

Colin N. Brooks <cnbrooks@mtu.edu>

Mon, Apr 23, 2012 at 11:07 AM

To: rchoensh@mtu.edu

Absolutely - the images are all yours to use, thanks for checking. Talk with you at 3:30 pm.

- Colin

Colin N. Brooks

Research Scientist, Environmental Science Lab Manager

Michigan Tech Research Institute (MTRI)

3600 Green Ct., Suite 100

Ann Arbor, Ml 48105

Ph: 734-913-6858 Cell: 734-604-4196

unw. mtri.org

unw. mtri. org/unpaved

mww. glosaocmapping.org

Sent from my Verizon Wireless 4G LTE DROID

-----Original message----- 


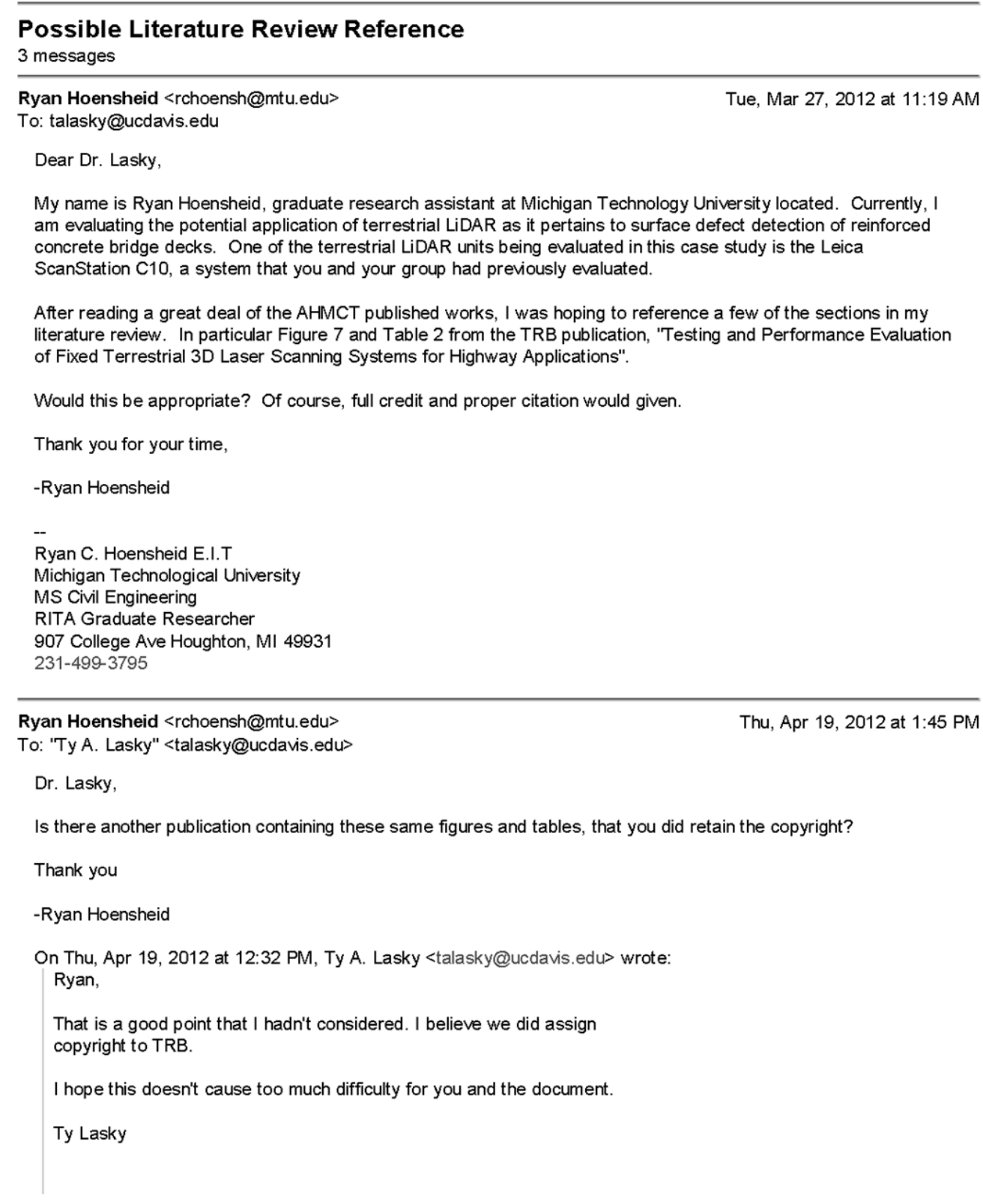




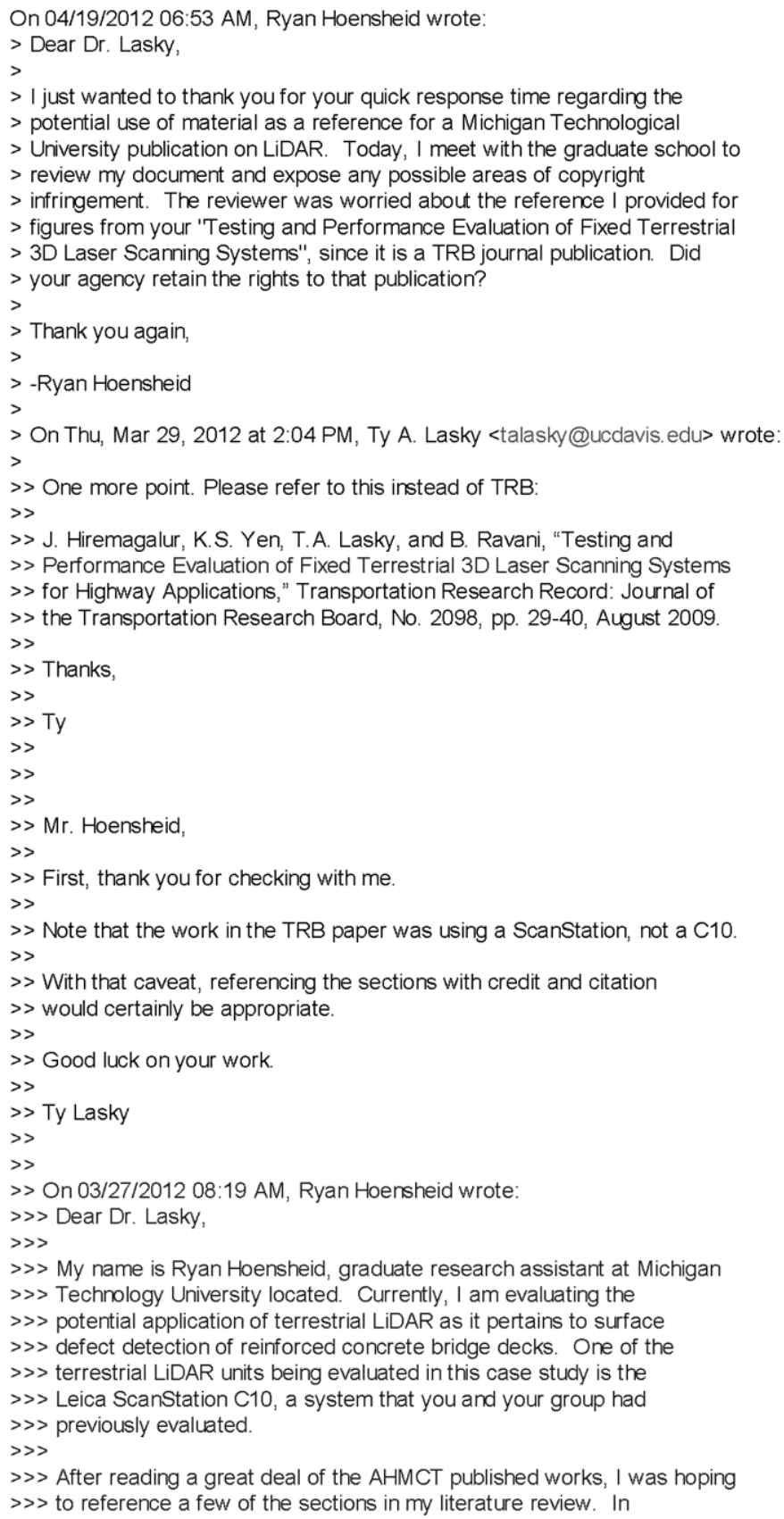


Michigan Technological University Mail - Possible Literature Review Re... https://mail.google.com/mail/u/0/?ui=2\&ik=40b9a1le7d\&view=pt\&searc..

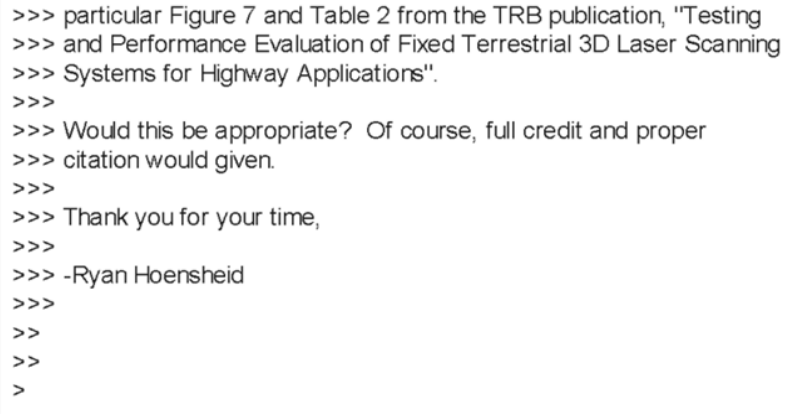

Ty A. Lasky <talasky@ucdavis.edu>

Thu, Apr 19, 2012 at 2:22 PM To: Ryan Hoensheid <rchoensh@mtu. edu>

Yes.

J. Hiremagalur, K.S. Yen, K. Akin, T. Bui, T.A. Lasky, and B. Ravani,

"Creating Standards and Specifications for the Use of Laser Scanning in

Caltrans Projects," Advanced Highway Maintenance and Construction

Technology Research Center Report UCD-ARR-07-06-30-01, June 30, 2007.

http://ahmct. ucdavis. edu/pdf/UCD-ARR-07-06-30-01-B. pdf

Hope that helps

Ty

[Quoted text hidden] 